\title{
A more efficient method to generate integration-free human iPS cells.
}

\section{AUTHOR(S):}

Okita, Keisuke; Matsumura, Yasuko; Sato, Yoshiko; Okada, Aki; Morizane, Asuka; Okamoto, Satoshi; Hong, Hyenjong; ... Takahashi, Jun; Saji, Hiroh; Yamanaka, Shinya

\section{CITATION:}

Okita, Keisuke ... [et al]. A more efficient method to generate integrationfree human iPS cells.. Nature methods 2011, 8: 409-412

\section{ISSUE DATE:}

2011-04-03

\section{URL:}

http://hdl.handle.net/2433/139494

\section{RIGHT:}

(C) 2011 Nature America, Inc. All rights reserved.; 許諾条件により本文は 2011-10-03に公開.; この論文は出版社版でありません。引用の際には 出版社版をご確認ご利用ください。; This is not the published version. Please cite only the published version. 
A more efficient method to generate integration-free human iPS cells

Keisuke Okita $^{1}$, Yasuko Matsumura ${ }^{1}$, Yoshiko Sato ${ }^{1}$, Aki Okada ${ }^{1}$, Asuka Morizane ${ }^{1,2}$, Satoshi Okamoto ${ }^{3}$, Hyenjong Hong ${ }^{1}$, Masato Nakagawa ${ }^{1}$, Koji Tanabe ${ }^{1}$, Ken-ichi Tezuka ${ }^{4}$, Toshiyuki Shibata ${ }^{5}$, Takahiro Kunisada ${ }^{4}$, Masayo Takahashi ${ }^{1,3}$, Jun Takahashi $^{1,2}$, Hiroh Saji ${ }^{6} \&$ Shinya Yamanaka ${ }^{1,7-9}$

${ }^{1}$ Center for iPS Cell Research and Application, Kyoto University, Kyoto, Japan.

${ }^{2}$ Department of Biological Repair, Institute for Frontier Medical Sciences, Kyoto University, Kyoto, Japan.

${ }^{3}$ Laboratory for Retinal Regeneration, Center for Developmental Biology, RIKEN, Kobe, Japan.

${ }^{4}$ Department of Tissue and Organ Development, Gifu University Graduate School of Medicine, Gifu, Japan.

${ }^{5}$ Department of Oral and Maxillofacial Science, Gifu University Graduate School of Medicine, Gifu, Japan.

${ }^{6}$ Human Leukocyte Antigen (HLA) Laboratory, Kyoto, Japan.

${ }^{7}$ Institute for Integrated Cell-Material Sciences, Kyoto University, Kyoto, Japan.

${ }^{8}$ Yamanaka Induced Pluripotent Stem Cell Project, Japan Science and Technology Agency, Kawaguchi, Japan.

${ }^{9}$ Gladstone Institute of Cardiovascular Disease, San Francisco, California, USA.

Correspondence should be addressed to K.O. (okita@cira.kyoto-u.ac.jp) and S.Y. (yamanaka@cira.kyoto-u.ac.jp). 
We report a simple method to generate human induced pluripotent stem cells (iPSCs) with episomal plasmid vectors. We generated human iPSCs from multiple donors, including two putative human leukocyte antigen (HLA)-homozygous donors who match $\sim 20 \%$ of the Japanese population at major HLA loci; most iPSCs integrated transgene-free and effectively differentiated in vivo and in vitro. This method may provide iPSCs suitable for autologous and allologous stem-cell therapy in the future.

Genomic integration of transgenes increases the risk of tumor formation and mortality in chimeric and progeny mice derived from induced pluripotent stem cells (iPSCs) ${ }^{1}$. Integration-free human iPSCs have been generated using several methods, including adenovirus ${ }^{2}$, Sendai virus ${ }^{3}$, the piggyBac system $^{4}$, minicircle vector ${ }^{5}$, episomal vectors ${ }^{6}$, direct protein delivery ${ }^{7}$ and synthesized mRNA $^{8}$ (Supplementary Table 1). However, reprogramming efficiency using integration-free methods is impractically low in most cases. Direct delivery of proteins or RNA is labor-intensive, requiring repeated delivery of the reprogramming factors. Modifying Sendai virus vectors or preparing synthesized RNA are technically demanding.

In the original report describing episomal plasmid vectors for reprogramming, the authors used seven factors, including POU5F1 (also known as OCT3/4), SOX2, KLF4, MYC (also known as c-MYC), NANOG, LIN28A (also known as LIN28) and SV40 large T antigen (SV4OLT), in three different vector combinations ${ }^{6}$ (T1 T3 combination, Fig. 1a and Supplementary Table 2). In this study, we used two findings from our laboratory to enhance efficiency of reprogramming by episomal plasmids: iPSC generation is markedly enhanced by p53 suppression ${ }^{9}$, and L-MYC is more potent and specific than c-MYC during human iPSC generation ${ }^{10}$.

We prepared four vector combinations (Fig. 1a and Supplementary Table 2). The Y1 combination had six factors (OCT3/4, SOX2, KLF4, c-MYC, LIN28 and NANOG) in three episomal plasmids. The Y2 combination contained an additional TP53 (also known as p53) shRNA in one of the three plasmids. We replaced $c-M Y C$ and NANOG with MYCL1 (also known as L-MYC) in the Y1 and Y2 combinations, respectively, to yield the Y3 and Y4 combinations (Fig. 1b).

We electroporated these seven combinations of episomal vectors (Y1-Y4 or T1-T3) into three human dermal fibroblast (HDF) lines and two dental pulp cell lines on day 0 (Fig. 1c). We trypsinized transfected cells on day 7 and reseeded them onto feeder layers. We maintained the cells in embryonic stem cell (ESC) medium, and small cell colonies became visible 2 weeks after transfection. We 
counted the number of colonies with a flat human ESC-like morphology and non-ESC-like colonies around day 30 (Supplementary Fig. 1). The Y4 combination resulted in significantly more iPSC colonies than did any of T1-T3 combinations (Fig. 1d). In addition to these five parental cell lines, we obtained iPSC colonies from seven additional HDF lines with the Y4 combination of factors (Supplementary Table 3).

We expanded ESC-like colonies derived with the Y4 combination for additional experiments. The majority of the colonies were expandable and exhibited a cellular morphology similar to that of human ESCs, characterized by large nuclei and scant cytoplasm (Fig. 2a,b). We termed these episomal plasmid vector-derived iPSCs 'pla-iPSCs'. Ten of eleven clones we analyzed were karyotypically normal (Supplementary Fig. 2 and Supplementary Table 4). Short tandem repeat analyses confirmed that pla-iPSC clones were derived from HDFs and dental pulp cells (Supplementary Table 5). Reverse transcription-PCR (RT-PCR) analyses revealed that pla-iPSC clones expressed pluripotent stem cell markers, such as OCT3/4, SOX2, NANOG and DPPA5, at levels comparable to those in ESCs and retrovirus-derived iPSC clones (Fig. 2c and Supplementary Figs. 3a, 4 and 5). Global gene expression profiles also showed that pla-iPSC clones were similar to ESC and retro-iPSC clones (Supplementary Fig. 6 and Supplementary Table 6). The DNA methylation levels of CpG sites in the promoter region of NANOG were high in parental HDFs and dental pulp cells but were low in pla-iPSCs and ESCs (Fig. 2d).

To examine whether episomal vectors persisted in pla-iPSCs, first we transfected an episomal vector encoding EGFP into fibroblasts and monitored fluorescence. Sixty-eight percent of the cells were fluorescent 1 week after transfection (Supplementary Fig. 7). However, the signal quickly decreased thereafter, and only $2.4 \%$ of cells were fluorescent 4 weeks after electroporation. Then we estimated the copy numbers of the episomal vectors in established pla-iPSC clones. We designed a PCR primer pair for EBNA-1 to calculate the copy numbers of the episomal vectors and another primer pair for the endogenous FBXO15 locus to estimate the cell number. We detected $\sim 200$ copies of the episomal vectors per cell $6 \mathrm{~d}$ after transfection (Fig. 2e and Supplementary Fig. $3 \mathbf{b}$ ). In contrast, we detected no EBNA-1 DNA in five of seven clones tested at passages 11-20 ( 80-120 d after transfection). The remaining two clones contained $\sim 0.001$ and 2 copies, respectively. The later clone likely had integrated the plasmid into a chromosome. These data demonstrated that the episomal vectors were spontaneously lost in the majority of pla-iPSC clones.

We examined the differentiation potential of pla-iPSCs in vivo. Injection of pla-iPSCs into the testes of immunodeficient mice yielded tumors within 3 months. Histological examination confirmed 
that these tumors were teratomas and contained tissues of all three germ layers, including neural epithelium, cartilage and gut-like epithelium (Supplementary Fig. 8).

We carried out directed differentiation of the pla-iPSCs into dopaminagic neurons in vitro (Online Methods). RT-PCR detected upregulation of SOX1, a marker of immature neural cells, and downregulation of OCT3/4 $12 \mathrm{~d}$ after induction (Supplementary Fig. 9a). Immunostaining showed that the majority of cells expressed Nestin after 29 d, with some cells still proliferating and expressed Ki67 (Supplementary Fig. 9b-e). Clusters of Nestin-expressing cells expressed PAX6, and more mature cell clusters expressed tyrosine hydroxylase, a marker of dopaminergic neurons (Supplementary Fig. 9f,g). Tyrosine hydroxylase-expressing cells localized with the neural markers Tuj1 and MAP2ab, and the vesicular monoamine transporter VMAT2 (Fig. 2f-h and Supplementary Fig. 9h-l). Therefore, pla-iPSCs have the potential to differentiate into dopaminergic neurons.

We also examined whether pla-iPSC clones differentiated into retinal pigment epithelial cells using a modified stromal cell-derived inducing activity method (Online Methods). Five of six pla-iPSC clones developed pigmented cell clusters after $30 \mathrm{~d}$ in conditioning medium of mouse PA6 stromal cells. The clusters grew and exhibited a squamous and hexagonal morphology, characteristic of retinal pigment epithelial cells (Fig. 2i,j).

We examined the human leukocyte antigen (HLA) types of our dental pulp-derived iPSC lines. In a previous study only one HLA type had been detected in two dental pulp lines by a PCR-reverse sequence-specific oligonucleotide probe (rSSOP) $\operatorname{protocol}^{11}$ : line DP74 had been typed as $H L A-A * 24$, -; HLA-B*52, -; HLA-DRB1*15, - and line DP94 as HLA-A*11, -; HLA-B*15, -; HLA-DRB1*04, ('-' means no other allele was detected; Supplementary Table 7). We also typed these lines with two additional analyses. A PCR-rSSOP protocol optimized for the Japanese population typed line DP74 and its progeny iPSC lines (454E-2 and 457C-1) as HLA-A*24:02, -; HLA-B*52:01, -; $H L A-D R B 1 * 15: 02,-$, and typed DP94 and its progeny iPSC line (453F-2) as HLA-A*11:01, -; $H L A-B * 15: 01,-; H L A-D R B 1 * 04: 06$, - . Sequence-based typing showed that the types of DP74 and DP94 were HLA-A*24:02:01, -; HLA-B*52:01:01, -; HLA-DRB1*15:02:01, - and HLA-A*11:01:01, -; HLA-B*15:01:01, -; HLA-DRB1*04:06:01, -, respectively. The families of the donors of the two dental pulp lines could not be typed because the lines were established in an anonymous way. Therefore, it is not possible to formally conclude that these donors are homozygous for the HLA haplotypes. Nevertheless, the fact that three independent analyses detected only one type in each donor is indicative of homozygosity. 
According to the HLA Laboratory database, frequencies of HLA-A*24:02; HLA-B*52:01; HLA-DRB1*15:02 and HLA-A*11:01; HLA-B*15:01; HLA-DRB1*04:06 haplotypes in the Japanese population are 8.5\% and 1.3\%, respectively, (http://www.hla.or.jp/hapro_e/top.html; Supplementary Table 8). Theoretically, iPSCs established from these two individuals match $20 \%$ of all the combinations of 2,117 haplotypes in Japanese population. Indeed, pla-iPSC lines derived from lines DP74 and DP94 match 32 of 107 donors $^{11}$ at the three HLA loci (HLA-A, HLA-B and HLA-DR) with the two-digit specification (Supplementary Table 7).

Others previously estimated that iPSC lines with 50 unique HLA homozygous haplotypes would match $~ 90 \%$ of the Japanese population at the HLA-A, HLA-B and HLA-DRB1 loci with two-digit specification ${ }^{12}$. We performed a similar estimation with four-digit specification using the HLA Laboratory database and found that 50 unique HLA-homozygous donors would cover 73\% of the Japanese population (Fig. 3a and Supplementary Table 8). Approximately 75 and 140 unique donors would be needed to cover $\sim 80 \%$ and $90 \%$, respectively. It would be necessary to type $\sim 37,000$, $\sim 64,000$ and $~ 160,000$ individuals, respectively, to identify these 50, 75 and 140 donors (Fig. 3b).

Allografts using HLA-homozygous iPSCs may provide a therapeutic alternative to autologous grafts, for cases in which transplant is likely to be needed soon after injury; furthermore, they allow for the advance selection of safe clones ${ }^{13}$. The beneficial effects of matching at major HLA loci are well documented in renal transplantation ${ }^{14,15}$, although recipients of allografts derived from HLA-homozygous iPSCs would still need immunosuppressants after transplantation because of other HLA antigens, non-HLA antigens and immunity by natural killer cells.

We report a simple, non-integrative method for reprogramming human cells. The increased efficiency and the use of nontransforming Myc should be useful to generate iPSCs from many donors, such as individuals with disease. The approach may also prove beneficial for generating human iPSCs for use in autologous and allologous stem cell therapy.

\section{Accession codes.}

Addgene: 27076 (pCXLE-hOCT3/4), 27077 (pCXLE-hOCT3/4-shp53-F), 27078 (pCXLE-hSK), 27079 (pCXLE-hMLN), 27080 (pCXLE-hUL), 27081 (pCXLE-Fbx15-cont2) and 27082 (pCXLE-EGFP).

\section{ACKNOWLEDGMENTS}

We thank K. Takahashi, T. Aoi, and Y. Yoshida for scientific discussion; M. Narita, T. Ichisaka, M. Ohuchi, M. Nishikawa and N. Takizawa for technical assistance; R. Kato, E. Nishikawa, S. Takeshima, 
Y. Ohtsu and H. Hasaba for administrative assistance; and H. Niwa (Riken) and J. Miyazaki (Osaka Univ.) for the CAG promoter. This study was supported in part by a grant from the Program for Promotion of Fundamental Studies in Health Sciences of National Institute of Biomedical Innovation, a grant from the Leading Project of Ministry of Education, Culture, Sports, Science and Technology (MEXT), a grant from Funding Program for World-Leading Innovative Research and Development on Science and Technology (FIRST Program) of Japan Society for the Promotion of Science, Grants-in-Aid for Scientific Research of Japan Society for the Promotion of Science and MEXT (to S.Y.), Grant-in-Aid for Young Scientists B (to K.O) and Senri Life Science Foundation (to K.O). H.H. is supported by a Japanese government (MEXT) scholarship.

\section{AUTHOR CONTRIBUTIONS}

K.O. and S.Y. conceived the project and wrote the manuscript. K.O. constructed the vectors with H.H., M. N., and K. Tanabe and conducted most of the experiments with Y.M., Y. S. and A.O. A.M. and J. T. carried out differentiation experiment into dopaminegic neurons. S. O. and M. T. performed differentiation into retinal pigment epithelial cells. K. Tezuka., T. S., and T. K. established dental pulp cell lines. H. S. performed HLA haplotyping in Japanese population and supervised HLA analysis.

\section{COMPETING FINANCIAL INTERESTS}

K.O., M.N. and S.Y. are filing a patent application to Japan, US and EU based on the results reported in this paper (PCT/JP2010/063733). S.Y. is a member of Scientific Advisory Board for iPS Academia Japan Inc. and iPierian Inc., which manage the patents.

\section{REFERENCES}

1. $\quad$ Okita, K., Ichisaka, T. \& Yamanaka, S. Nature 448, 313-317 (2007).

2. Zhou, W. \& Freed, C.R. Stem Cells 27, 2667-2674 (2009).

3. Fusaki, N., Ban, H., Nishiyama, A., Saeki, K. \& Hasegawa, M. Proc. Jpn. Acad. B 85, 348-362 (2009).

4. Woltjen, K. et al. Nature 458, 766-770 (2009).

5. Jia, F. et al. Nat. Methods 7, 197-199 (2010).

6. Yu, J. et al. Science 324, 797-801 (2009). 
7. Kim, D. et al. Cell Stem Cell 4, 472-476 (2009).

8. Warren, L. et al. Cell Stem Cell 7, 618-630(2010).

9. $\quad$ Hong, H. et al. Nature 460, 1132-1135 (2009).

10. Nakagawa, M., Takizawa, N., Narita, M., Ichisaka, T. \& Yamanaka, S. Proc. Natl. Acad. Sci. USA 107, 14152-14157 (2010).

11. Tamaoki, N. et al. J. Dent. Res. 89, 773-778 (2010).

12. Nakatsuji, N., Nakajima, F. \& Tokunaga, K. Nat. Biotechnol. 26, 739-740 (2008).

13. Tsuji, O. et al. Proc. Natl. Acad. Sci. USA 107, 12704-12709 (2010).

14. Takemoto, S.K., Terasaki, P.I., Gjertson, D.W. \& Cecka, J.M. N. Engl. J. Med. 343, 1078-1084 (2000).

15. Aydingoz, S.E. et al. Hum. Immunol. 68, 491-499 (2007).

\section{FIGURE LEGENDS}

\section{Figure 1}

Establishment of human iPSCs putatively homozygous for the HLA-A, HLA-B and HLA-DRB1 loci.

(a) Combinations of reprogramming factors and episomal vectors used in this study. (b) Episomal expression vectors in the Y4 combination. CAG, CAG promoter; WPRE, woodchuck hepatitis post-transcriptional regulatory element; and $p A$, polyadenylation signal. (c) Schematic of the pla-iPSC induction protocol. HDFs or dental pulp cells were cultured in Dulbecco's modified Eagle medium (DMEM) with 10\% (vol/vol) fetal bovine serum (FBS) or mesenchymal stem cell growth medium (MSCGM), respectively. The episomal vector mixture was electroporated into cells on day 0; transfected cells were collected and reseeded on feeders on day 7, culture medium was changed to ESC medium containing bFGF on day 8, and iPSC colonies were counted and picked around day 26-32. (d) Numbers of colonies per $1.0 \times 10^{5}$ cells obtained with different combinations of reprogramming factors. For negative control, we transduced episomal vector encoding EGFP (control). MEF, mouse embryonic fibroblasts; SNL, mouse embryonic fibroblast cell line. Data are means \pm s.d. of numbers of ESC-like colonies obtained from 15 independent induction experiments using five cell lines. ${ }^{* * * *} P$ $<0.05$ against T1, T2, T3 and control; $* * * P<0.05$ against T1, T3 and control; $* * P<0.05$ against T1 and control; $* P<0.05$ against control. 


\section{Figure 2}

Characterization of pla-iPSC clones. (a,b) Phase contrast images of an established pla-iPSC line. Scale bars, $1 \mathrm{~mm}$ (a) and $100 \mu \mathrm{m}$ (b). (c) RT-PCR analyses for pluripotent cell markers. Total RNA was isolated from pla-iPSC clones established with the Y1 (clone 454B-1), Y2 (454C-2), Y3 (454D-1) or Y4 (454E-2, 451F-3, 457C-1 and 453F-2) combinations, from retrovirus-derived iPSC clones (retro-iPSC) and from ESC lines. In the lanes labeled OCT3/4 and SOX2, PCR primers only detected endogenous gene expression; in the Ret-OCT lane, PCR primers specifically amplified the retroviral OCT3/4 transgene. GAPDH was used as a loading control. As a negative control, GAPDH amplification was also performed without RT reaction (RT (-)). Fibroblasts $4 \mathrm{~d}$ after electroporation of the Y4 mixture (HDF-elepo) were used as another negative control. (d) DNA methylation status of the NANOG promoter region in the indicated cell lines. Open and closed circles indicate unmethylated and methylated CpG dinucleotides, respectively. (e) Copy numbers of episomal vectors in pla-iPSC clones. Numbers in parentheses indicate passage number. Also shown are the estimated numbers of cells analyzed for each clone. Fibroblasts $6 \mathrm{~d}$ after electroporation of the Y4 combination were analyzed (fibro-d6) as a positive control. (f-h) Differentiation of pla-iPSC clone (454E-2) into dopaminegic neurons. Micrographs are immunostained for Tuj1 (f) and tyrosine hydroxylase (TH) (g). A merged image with nuclear staining using DAPI (h) is shown. Scale bars, $20 \mu \mathrm{m}$. (i,j) Differentiation of pla-iPS clone (454E-2) into retinal pigment epithelial cells. Scale bars, $100 \mu \mathrm{m}$ (i) and $50 \mu \mathrm{m}(\mathbf{j})$.

\section{Figure 3}

Estimated coverage of the Japanese population by HLA homozygous donors. (a) Estimated cumulative coverage of the Japanese population by theoretical unique HLA homozygous donors at HLA-A, HLA-B and HLA-DRB1 loci with four-digit specification. (b) Estimated numbers of donors required to identify individuals with unique HLA homozygous haplotypes.

\section{Online Methods}

\section{Cell culture.}

Human fibroblasts HDF1419, HDF1388, HDF1429, HDF1377, HDF1437 and HDF1554 were purchased from Cell Applications, Inc., and TIG121, TIG120, TIG114 and TIG107 were obtained from the Japanese Collection of Research Bioresources. Human fibroblasts were cultured in DMEM 
(Nacalai Tesque) supplemented with 10\% FCS (Invitrogen). Human dental pulp (DP) cells were established from human third molars as described previously ${ }^{11}$ and were maintained in mesenchymal stem cell growth medium (MSCGM; Lonza). Human ESC lines (KhES-1 and KhES-3) were obtained from Kyoto University. H1 and H9 were from WiCell Research Institute. Mouse embryonic fibroblasts (MEFs) were isolated from embryonic day 13.5 embryos of C57BL/6 mice. All mice used in this study were bred and killed appropriately following code of ethics of animal research committee in Kyoto University. MEF and SNL cells ${ }^{16}$ were cultured in DMEM supplemented with 7\% (vol/vol) FCS, 2 $\mathrm{mM}$ L-glutamine and 50 units and $50 \mathrm{mg} \mathrm{ml}^{-1}$ penicillin and streptomycin, respectively. Established iPSCs and ESCs were maintained on mitomycin C-treated SNL cells in primate ESC medium (ReproCELL) containing $4 \mathrm{ng} \mathrm{ml}^{-1}$ of bFGF (Wako) as described previously ${ }^{17}$.

\section{Vector construction.}

Efficient transgene expression was achieved by inserting the woodchuck hepatitis post-transcriptional regulatory element (WPRE) upstream of the polyadenylation signal of $\mathrm{pCX}-\mathrm{EGFP}^{18}$. The episomal cassette was transferred from pCEP4 (Invitrogen). The EBNA-1 sequence (EcoRI and MfeI sites) was flanked by two loxP sequences, and the loxP-EBNA-1-loxP-OriP cassette was then digested with BamHI and BglII and inserted into the BamHI site of pCX-EGFP containing the WPRE. This episomal vector was designated pCXLE-EGFP.

Human cDNAs encoding OCT3/4, SOX2, KLF4, C-MYC, L-MYC, NANOG and LIN28 were amplified by PCR and cloned into pCR2.1 (Invitrogen). The translation termination codons of SOX2, c-MYC, $L-M Y C$ or LIN28 were replaced with a BamHI site and then were also cloned into pCR2.1. The cDNAs without a translation termination codon were thereafter ligated with $2 A$ self-cleavage sequences in pBS-2A ${ }^{19}$ with appropriate restriction enzymes to generate pBS-cDNA-2A. $c-M Y C-2 A$ was digested with NotI and BspHI and was ligated into the NotI and NcoI sites of pBS-LIN28-2A in the same reading frame to generate the $c-M Y C-L I N 28-N A N O G$ cassette. These $c D N A-2 A$ or c-MYC-2A-LIN28-2A constructs were then ligated to another cDNA or NANOG in pCR2.1 with the translation termination codon in the same reading frame using appropriate restriction enzymes. These cDNA-2A-cDNA-stop or c-MYC-2A-LIN28-2A-NANOG-stop constructs were then inserted into the EcoRI site of pCXLE-EGFP. pCXLE-hOCT3/4-shp53 was constructed by inserting an shRNA expression cassette for $p 53$, driven by the mouse U6 promoter, into the BamHI site of pCXLE-hOCT3/4. The pCXLE-Fbx15-cont2 was generated by inserting the FBXO15 cDNA into pCXLE-EGFP. Episomal vectors described previously ${ }^{6}$ were obtained from Addgene (20922-20927). 


\section{Generation of iPSCs with episomal vectors.}

HDF and DP cells were cultured in DMEM supplemented with 10\% FBS and mesenchymal stem cell growth medium (MSCGM), respectively. Three micrograms of expression plasmid mixtures were electroporated into $6 \times 10^{5} \mathrm{HDF}$ or DP cells with Microporator (Invitrogen) with a $100-\mu \mathrm{l}$ kit according to the manufacturer's instructions. The plasmid mixtures used in the experiments are shown in Supplementary Table 2. Conditions used were 1,650 V, 10 ms, 3 time pulses for HDF, and 1,800 V, $20 \mathrm{~ms}, 1$ time pulse for DP cells. The cells were trypsinized $7 \mathrm{~d}$ after transduction, and $1 \times 10^{5}$ cells were re-plated onto 100-mm dishes covered with an SNL or MEF feeder layer. The culture medium was replaced the next day with Primate ESC medium (ReproCELL) containing bFGF. The colonies were counted 26-32 d after plating, and those colonies similar to human ESCs were selected for further cultivation and evaluation. The pla-iPSC clones used in this study are summarized in

\section{Supplementary Table 9.}

\section{Characterization of pla-iPSC clones.}

Isolation of total RNA, RT-PCR of marker gene expression, DNA microarray, bisulfite genomic sequencing and teratoma formation were performed as previously described ${ }^{17}$. The primer sequences used in this study are shown in Supplementary Table 10. The chromosomal G-band analyses were performed at the Nihon Gene Research Laboratories. Short tandem repeat analyses were performed at Bex Co.; briefly, genomic DNAs were amplified by the PowerPlex 16 system (Promega) and were then analyzed with an ABI PRISM 3100 genetic analyzer and the GeneMapper v3.5 software program (Applied Biosystems). Differentiation of pla-iPSC clones into dopaminergic neurons was performed using the serum-free culture of embryoid body-like aggregates (SFEB) method combined with double SMAD inhibition by a BMP antagonist and an Activin/Nodal inhibitor as described elsewhere ${ }^{20}$. In vitro directed differentiation into retinal pigment epithelial cells was performed with the modified stromal cell-derived inducing activity method ${ }^{21,22}$. Briefly, pla-iPSCs were collected with trypsin and collagenase IV, and were treated with inhibitors for WNT and Nodal signaling under serum-free conditions. The cells were then maintained in PA6-conditioning medium for maturation.

\section{Episomal copy-number detection.}


Cells cultured in 60-mm dishes were collected with a cell scraper after removing feeder cells with treatment of CTK dissociation solution. The cells were then placed into tubes and centrifuged, and the cell pellets were lysed with $200 \mu \mathrm{l}$ of lysis solution, containing $1 \times$ Ex Taq buffer (Takara) and $167 \mu \mathrm{g}$ $\mathrm{ml}^{-1}$ proteinase $\mathrm{K}$. The lysates were incubated at $55^{\circ} \mathrm{C}$ for $3 \mathrm{~h}$, and proteinase $\mathrm{K}$ was inactivated at $95{ }^{\circ} \mathrm{C}$. The lysates were used for quantitative PCR analysis. The pCXLE-hFbx15-cont2 plasmid was used to generate a standard curve to determine the correlation between copy number and threshold cycle (Ct) values for FBXO15 or EBNA-1. Then the copy number of FBXO15 and EBNA-1 in each iPSC sample was estimated from the observed Ct values. The cell number in each reaction was estimated by dividing the estimated copy number of FBXO15 by two since each cell had two FBXO15 alleles. One reaction included up to $1.2 \times 10^{4}$ cells. The total copy number of EBNA-1 was measured in $\sim 5 \times 10^{4}$ cells by repeating six or seven reactions.

\section{HLA typing and estimation of coverage.}

HLA typing of 107 DP cell lines was performed with the PCR-reverse sequence specific oligonucleotide probe (rSSOP) method using LABType SSO (One lambda) at Repro Cell ${ }^{23}$. Additional HLA typing was performed with PCR-rSSOP using WAKFlow (Wakunaga Pharmaceutical Co.) at HLA Laboratory. We performed pedigree study of 4,743 Japanese families (17,325 members) and identified 2,117 haplotypes, including interlocus recombinant haplotypes, which were detected in family studies. The haplotype frequency was calculated by direct counting on the parents in the families. Sequence-based typing was performed with AlleleSEQR (Atria Genetics) at Mitsubishi Chemical Medience Corporation.

To estimate coverage of Japanese population by HLA homozygous donors, we first calculated the frequencies of all possible combinations of the 2,117 HLA haplotypes shown in Supplementary Table 8. Haplotype combinations that can be covered by a given homozygous donor were then identified and their frequencies were added to estimate coverage by the homozygous donor. When one HLA-A, HLA-B, HLA-DRB1 heterozygous individual was covered by multiple homozygous donors, we counted only once to avoid overestimation.

The expected number (EN) of each homozygous haplotype at a given population size (PS) was first calculated as; $\mathrm{EN}=(\text { haplotype frequency })^{2} \times$ PS. EN $($ if EN $<1)$ or 1 was then summed for each homozygous haplotype to estimate the expected numbers of unique HLA haplotype donors at the given PS. 


\section{Statistical analyses.}

Data are shown as the mean \pm s.d. Statistical significance among multiple groups was evaluated with the Steel-Dwass test.

\section{REFERENCES}

16. McMahon, A.P. \& Bradley, A. Cell 62, 1073-1085 (1990).

17. Takahashi, K. et al. Cell 131, 861-872 (2007).

18. Niwa, H., Yamamura, K. \& Miyazaki, J. Gene 108, 193-199 (1991).

19. Okita, K., Nakagawa, M., Hyenjong, H., Ichisaka, T. \& Yamanaka, S. Science 322, 949-953 (2008).

20. Morizane, A., Doi, D., Kikuchi, T., Nishimura, K. \& Takahashi, J. J. Neurosci. Res. 89, 117-126 (2010).

21. Kawasaki, H. et al. Proc. Natl. Acad. Sci. USA 99, 1580-1585 (2002).

22. Osakada, F. et al. J. Cell Sci. 122, 3169-3179 (2009).

23. Itoh, Y. et al. Immunogenetics 57, 717-729 (2005). 
Figure 1, Okita et al.

a

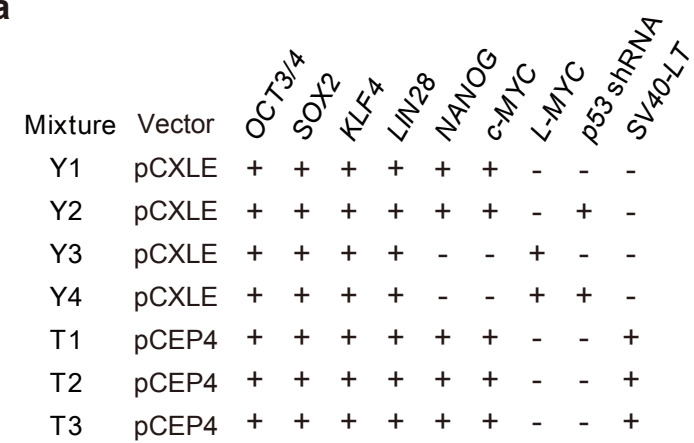

d

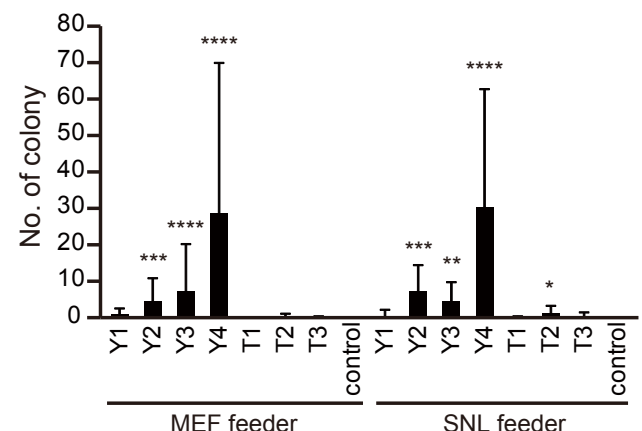

b

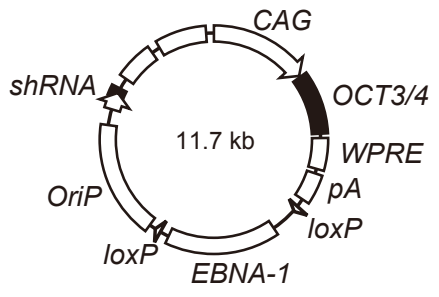

pCXLE-hOCT3/4-shp53

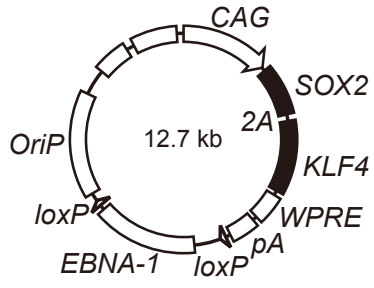

pCXLE-hSK

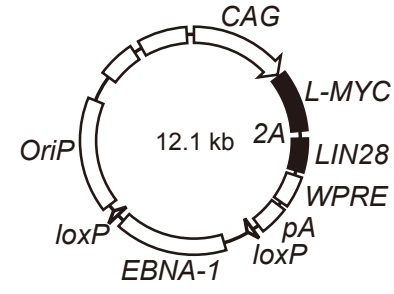

pCXLE-hUL

C

$\begin{gathered}\text { Plasmid } \\
\text { transduction }\end{gathered}$
\begin{tabular}{|l|lc|}
$\begin{array}{c}\text { Reseeding } \\
\text { on feeder }\end{array}$ & $\begin{array}{c}\text { Colony } \\
\text { picking up }\end{array}$ \\
\hline $10 \%$ FBS or MSCGM & ES medium + bFGF & $\mathrm{d} 26-32$ \\
$\mathrm{~d} 0$ & $\mathrm{~d} 7 \mathrm{~d} 8$ &
\end{tabular}


Figure 2, Okita et al.
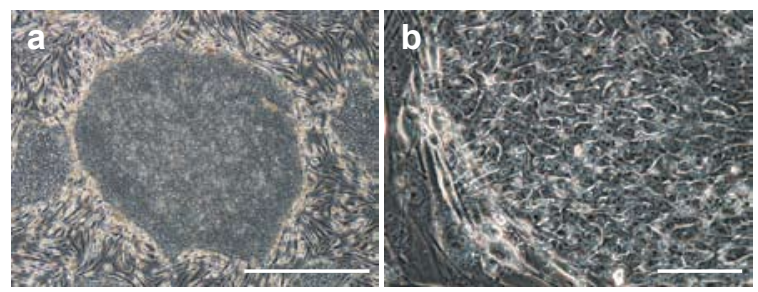

c

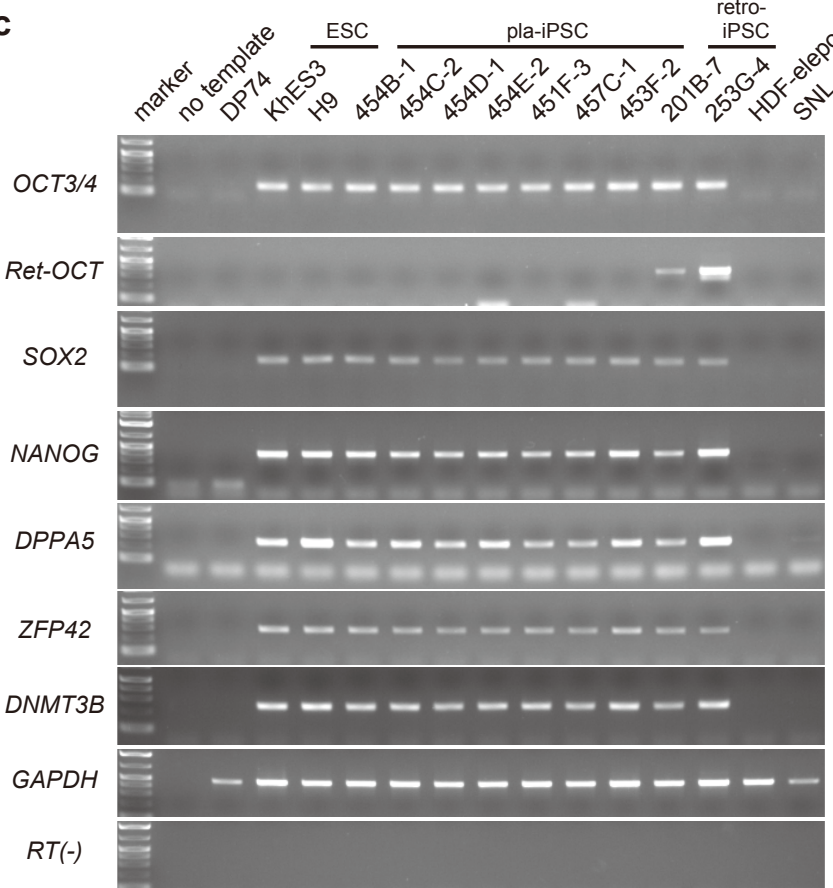

d

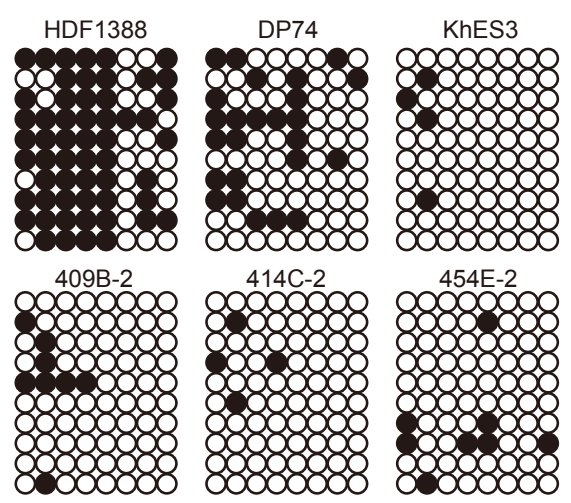

e

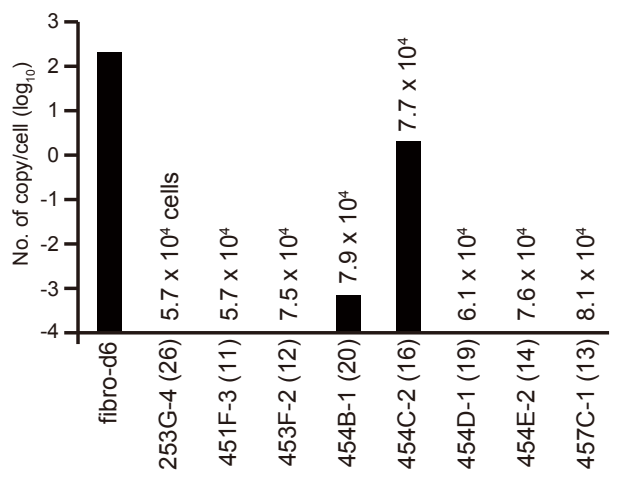

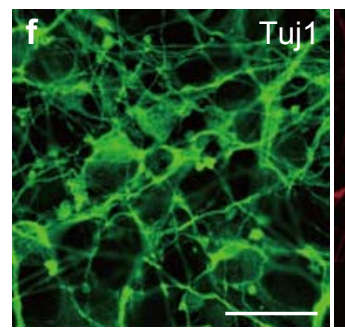
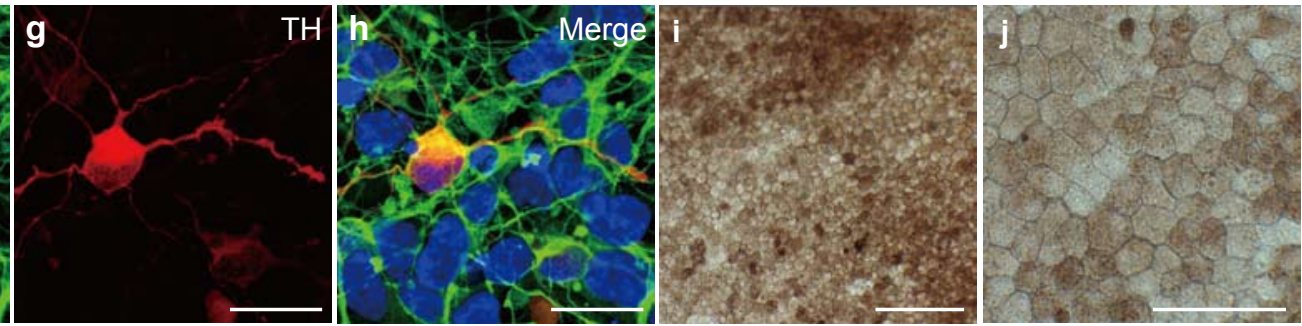
Figure 3, Okita et al.

a

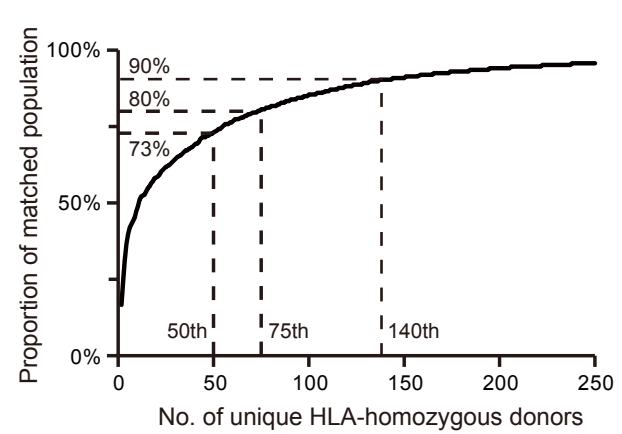

b

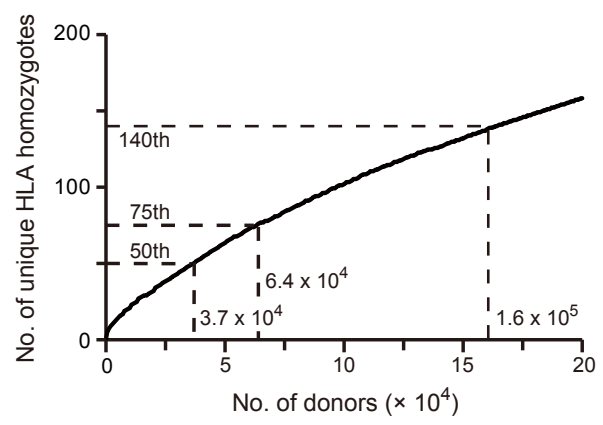



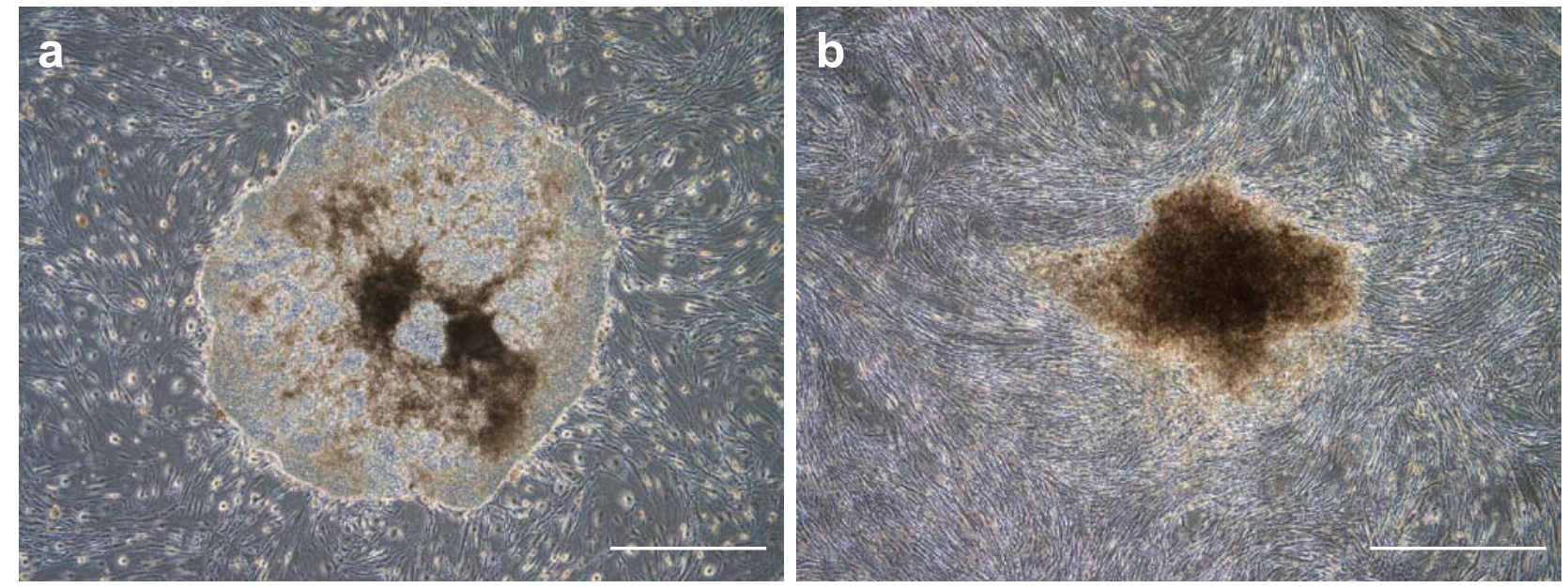

Supplementary Figure 1. Morphology of induced colonies.

(a) Morphology of an ESC-like colony. Bar $=1 \mathrm{~mm}$. (b) Morphology of a non-ESC-like colony. Bar $=1 \mathrm{~mm}$. 


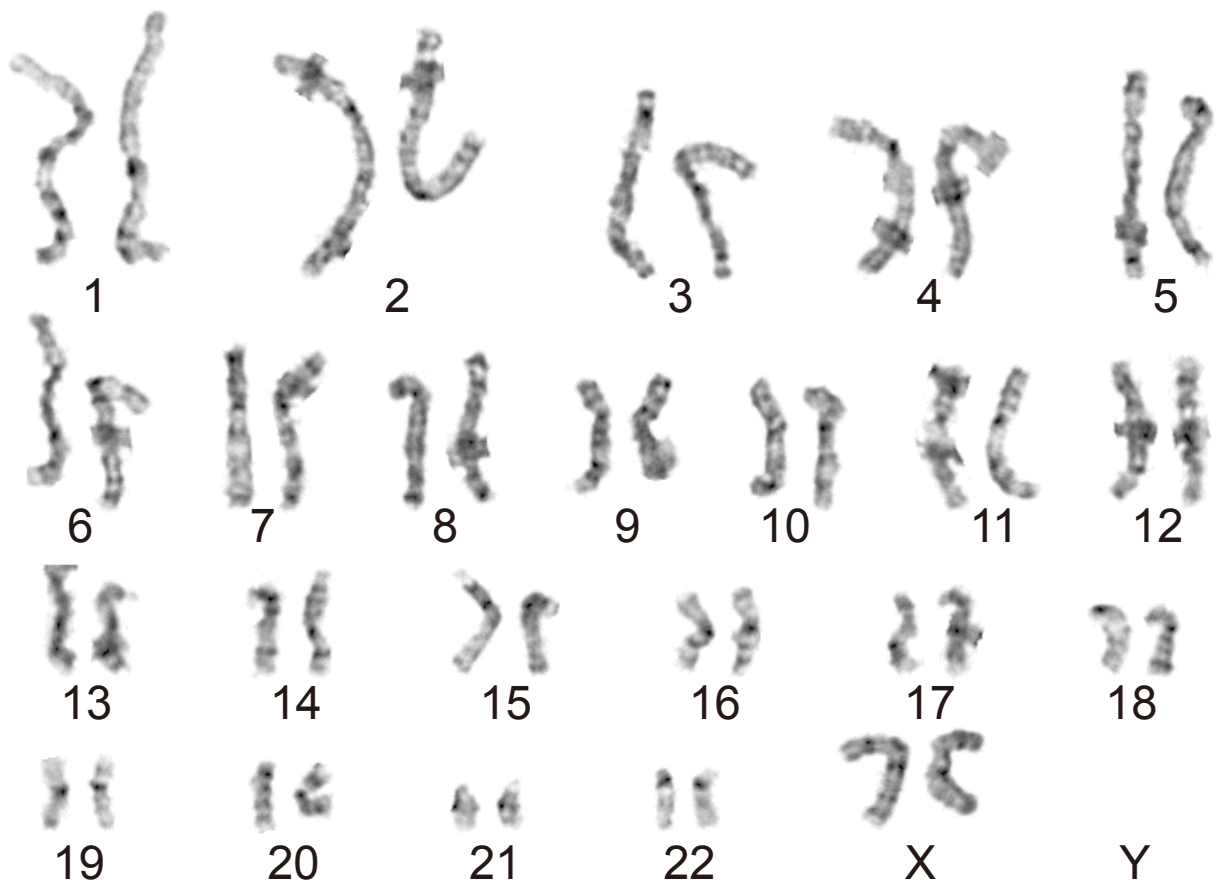

Supplementary Figure 2. G-band staining of a pla-iPSC clone (409B-2). 
a

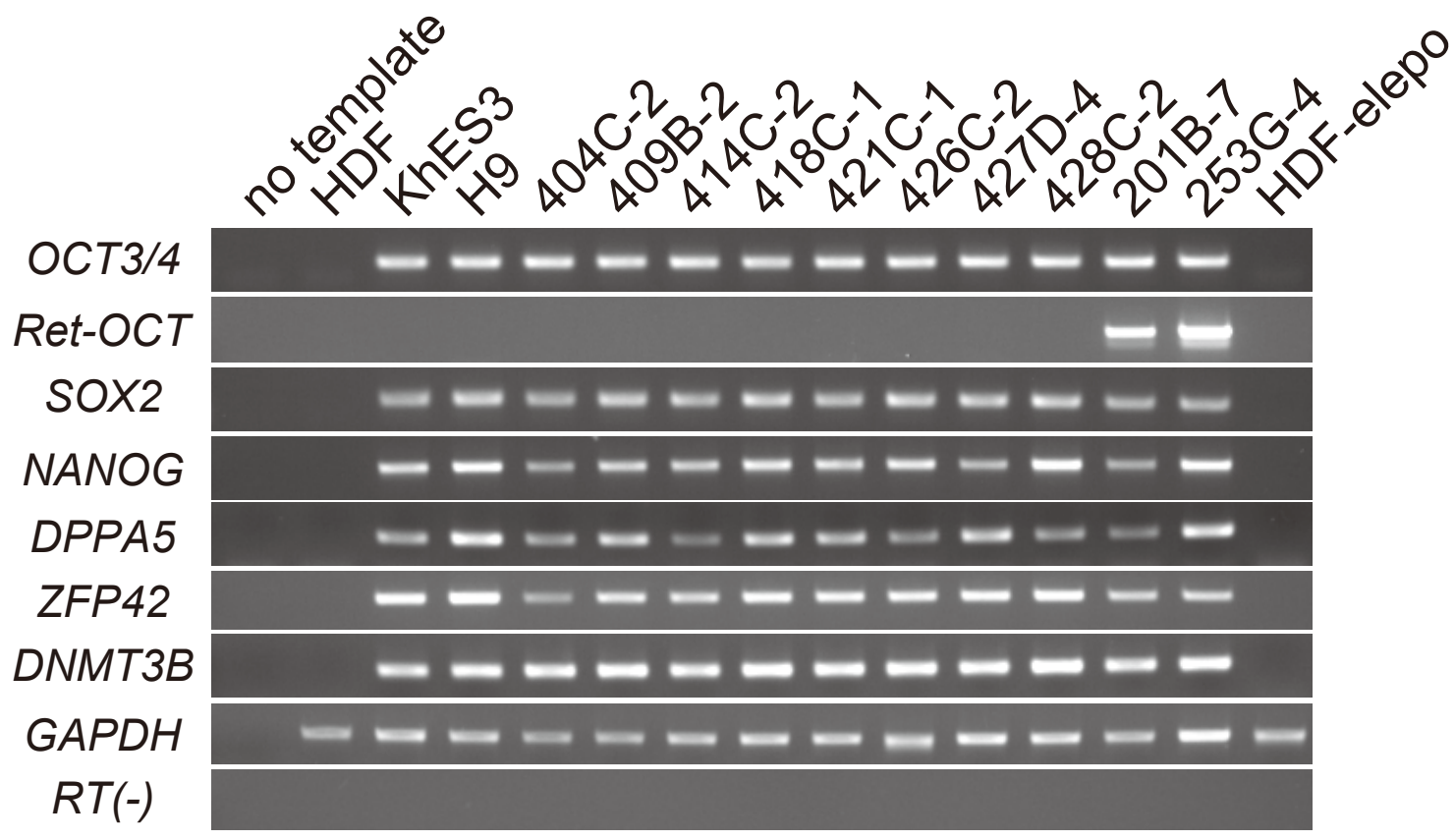

b

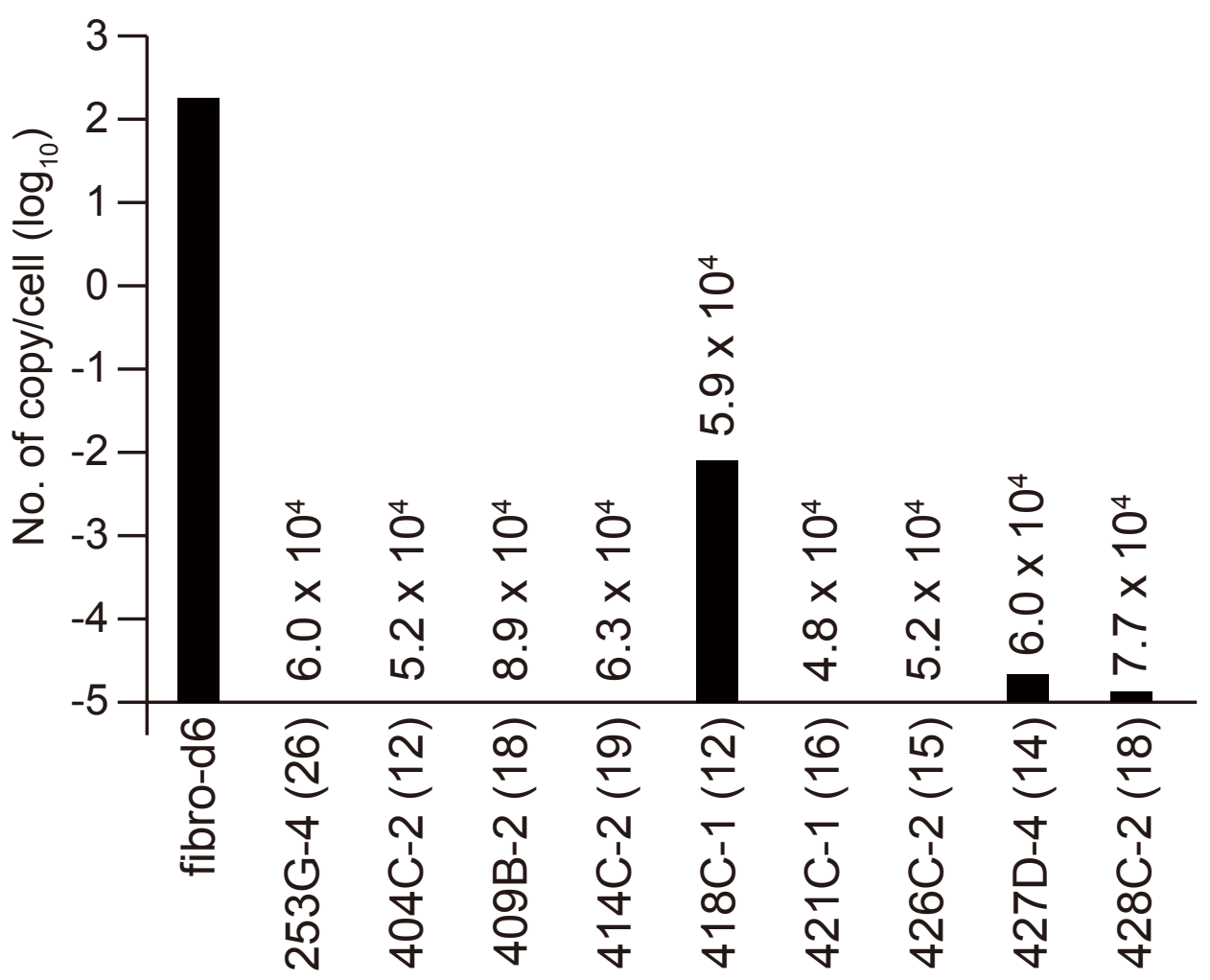

Supplementary Figure 3.

Gene expression and copy number of the episomal plasmids in fibroblast-derived pla-iPSCs.

(a) Expressions of pluripotent cell marker genes by an RT-PCR analysis. Pla-iPSCs established with the Y4 mix from various fibroblasts (404C-2, 409B-2, 414C-2, 418C-1, 421C-1, 426C-2, 427D-4, and 428C-2), retrovirus-derived iPSCs (201B-7 and 253G-4), and ESCs (KhES-3 and H9) were used for the analyses. Endogenous OCT3/4 (OCT3/4),

retrovirus-derived OCT3/4 (Ret-OCT), and endogenous SOX2 (SOX2) were examined. GAPDH was examined as a loading control. For the control, fibroblasts were examined 4 days after electroporation of Y4 mixture (HDF-elepo). (b) Copy numbers of episomal vectors that remained in the Pla-iPSC clones. Established clones were harvested, lysed, and used for the quantitative PCR analyses. In several clones, including 404C-2 and 409B-2, we were unable to detect any episomal vectors. The numbers in parentheses indicate the passage number. The examined cell numbers are also indicated. For the control, retrovirus-derived iPSC clone (253G-4) and fibroblasts at 6 days after electroporation of Y4 mixture (fibro-d6) were analyzed. 
OCT3/4
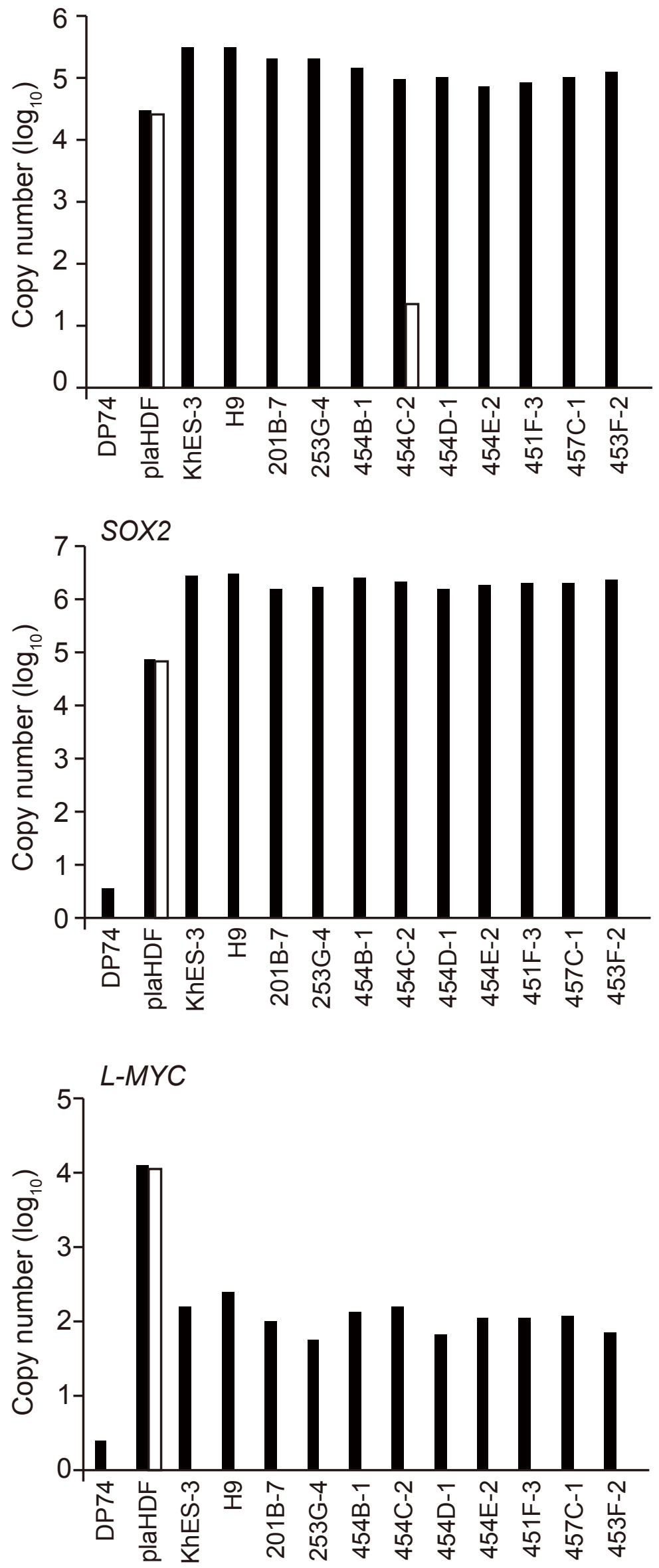

\section{GAPDH}
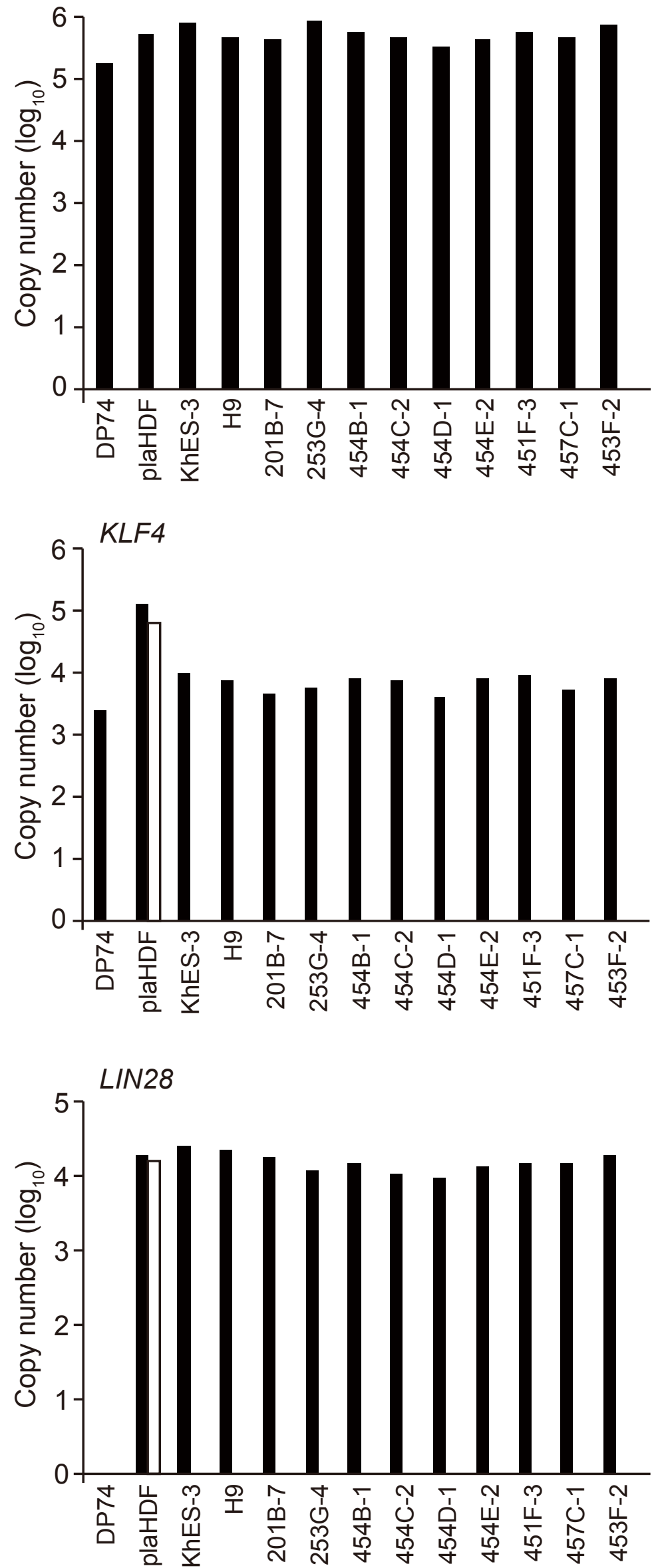

Supplementary Figure 4. Quantitative PCR for detection of transgenic expression in DP-derived pla-PSCs.

Total RNA was isolated from ESCs (KhES-3 and H9), retrovirus-derived iPSCs (201B-7 and 253G-4), and pla-iPSCs (454B-1 established by Y1 mix, 454C-2 by Y2, 454D-1 by Y3, 454E-2, 451F-3, 457C-1, and 453F-2 by Y4), and were used for the RT-PCR analyses. White columns show the copy numbers obtained with specific primers to the episomal vector of the indicated genes. Black columns show the copy numbers obtained with primers for the coding sequence, which could detect both endogenous and episomal vector-derived transcripts. For the controls, fibroblasts at 4 days after electroporation of $Y 4$ mixture were analyzed (plaHDF). 

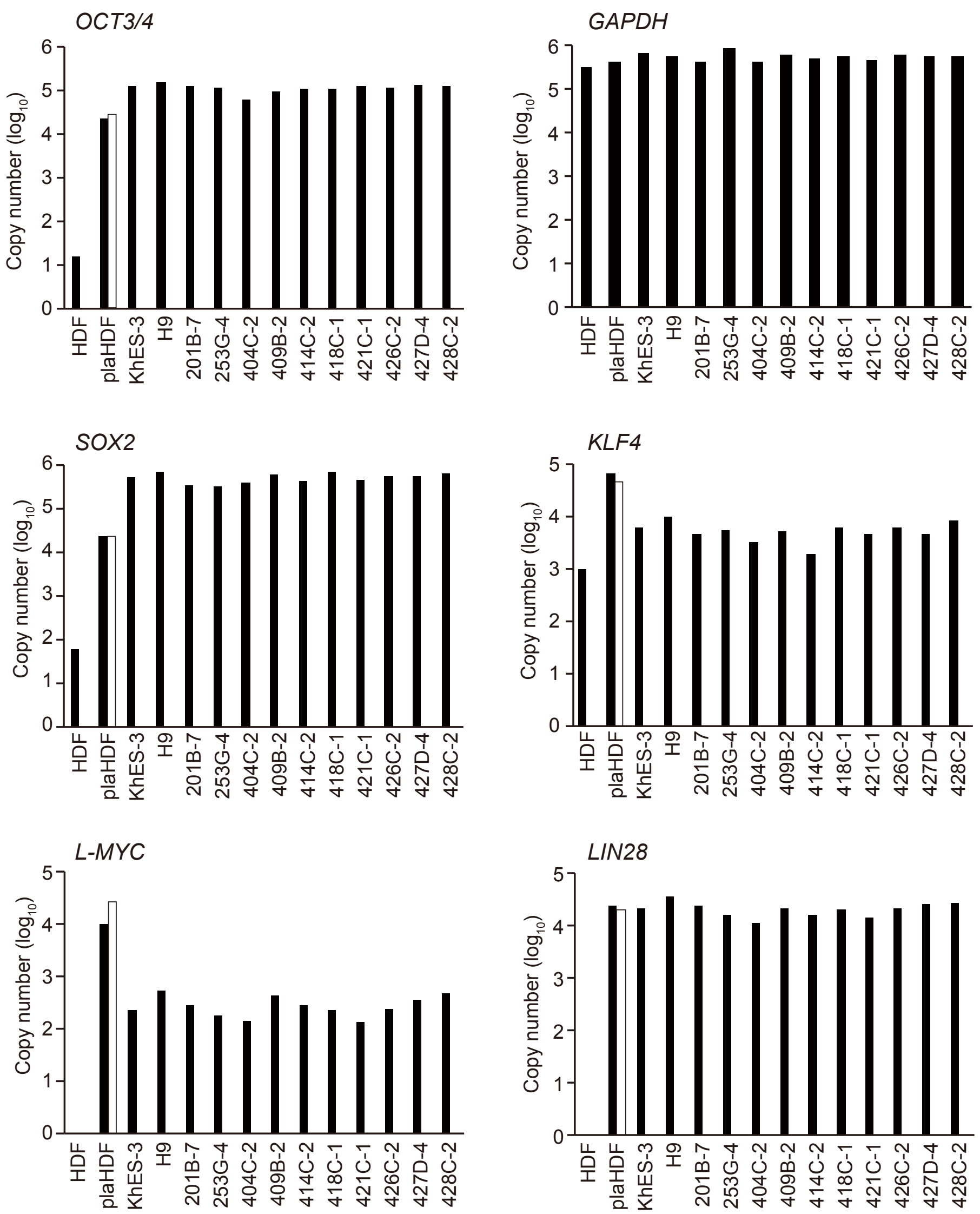

Supplementary Figure 5. Quantitative PCR for the detection of transgene expression in fibroblast-derived pla-iPSCs.

Total RNA was extracted from ESCs (KhES-3 and H9), retrovirus-derived iPSCs (201B-7 and 253G-4), and pla-iPSCs (404C-2, 409B-2, 414C-2, 418C-1, 421C-1, 426C-2, 427D-4, and 428C-2), and were used for the RT-PCR analyses. White columns show the copy numbers obtained with specific primers against the episomal vector of the indicated genes. Black columns show the copy numbers obtained with primers for the coding sequence, which could detect both endogenous and episomal vector-derived transcripts. As a control, fibroblasts at 4 days after electroporation of $Y 4$ mixture were analyzed (plaHDF). 


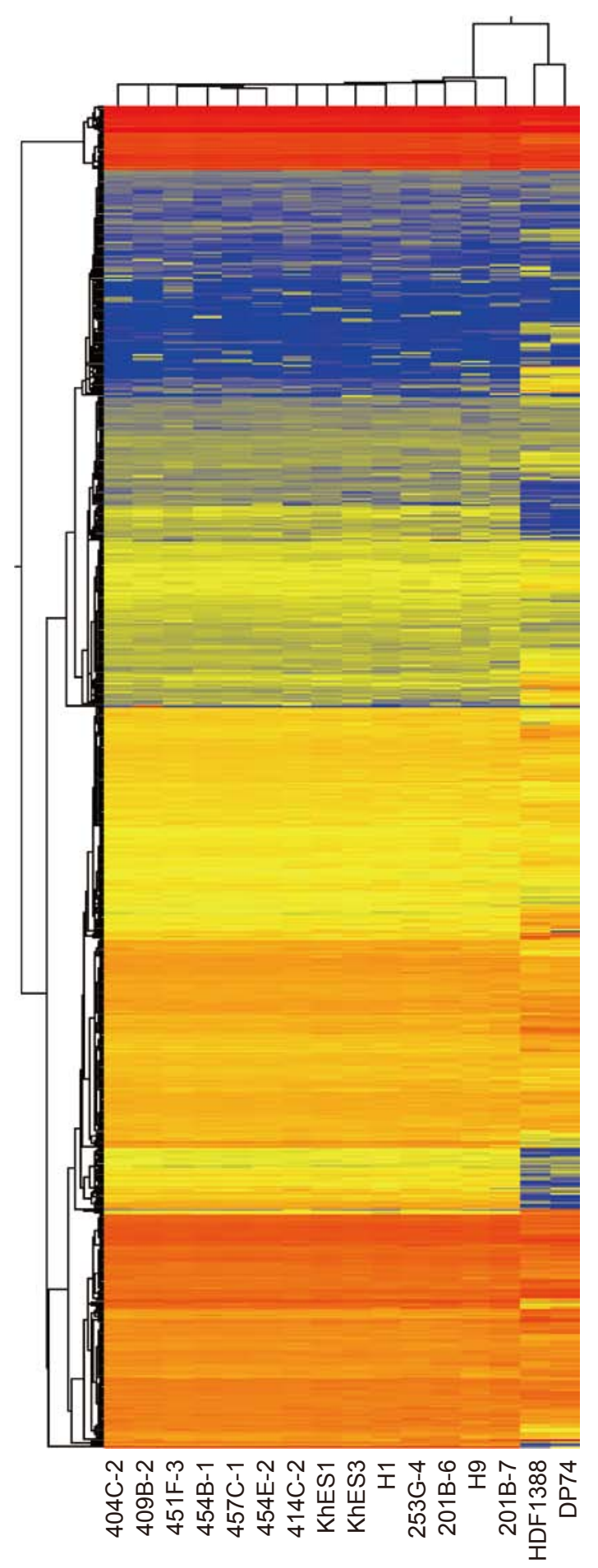

Supplementary Figure 6. Comparison of the global gene expression profiles.

Unsupervised clustering was performed using microarray data from ESC clones (KhES1, KhES3, $\mathrm{H} 1$, and H9), pla-iPSC clones (404C-2, 409B-2, 414C-2, 451F-3, 454B-1, 454E-2, and 457C-1), retrovirus-derived iPSC clones (201B-6, 201B-7, and 253G-4), fibroblasts (HDF1388), and DP cells (DP74). 
Phase
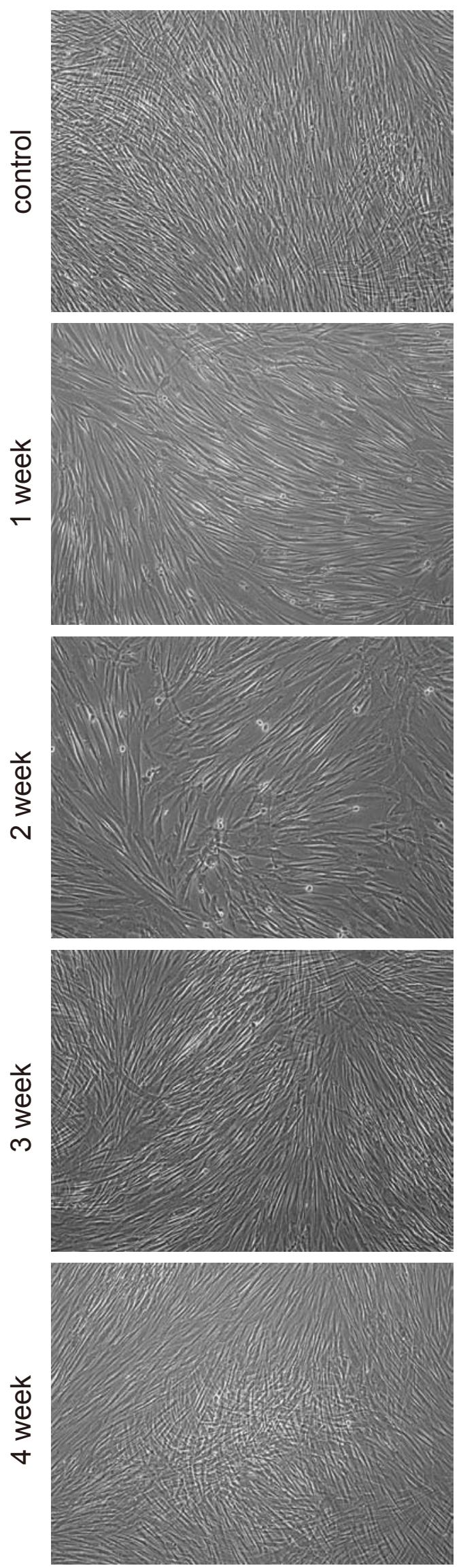

Fluorescence
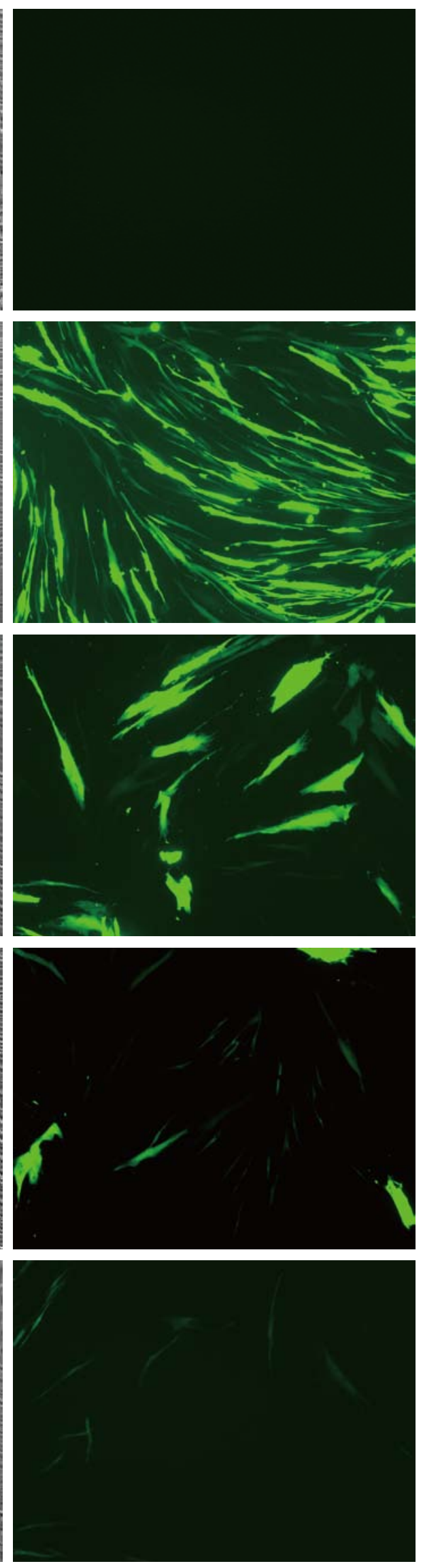

FACS
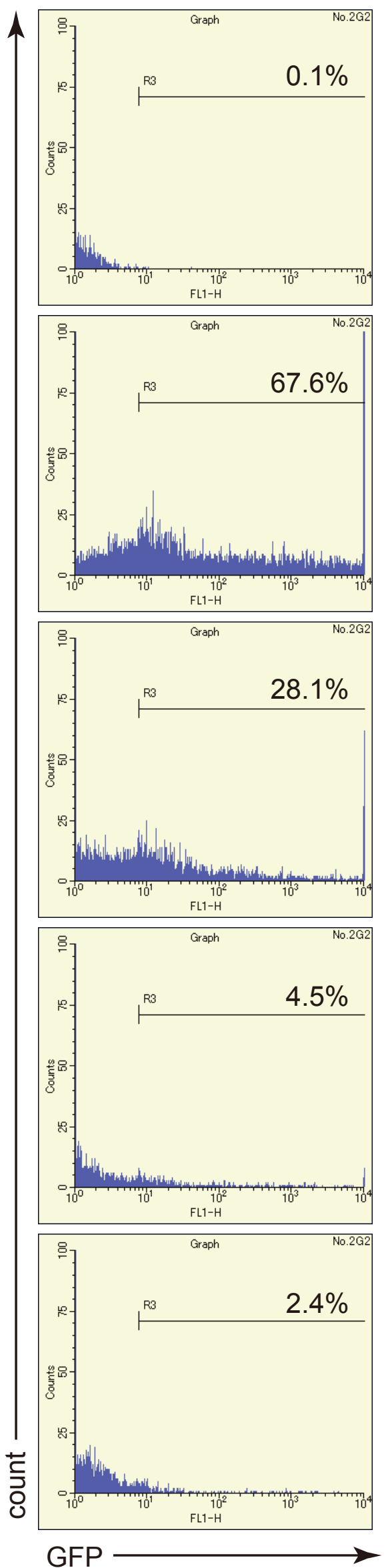

Supplementary Figure 7. Expression of GFP fluorescence from episomal vector.

Human fibroblasts were transduced with an episomal vector encoding GFP via electroporation. The cells were photographed and analyzed by flow cytometry at 1, 2, 3, and 4 weeks after transduction. 
Neural tissue

Cartilage

Gut-like epithelium
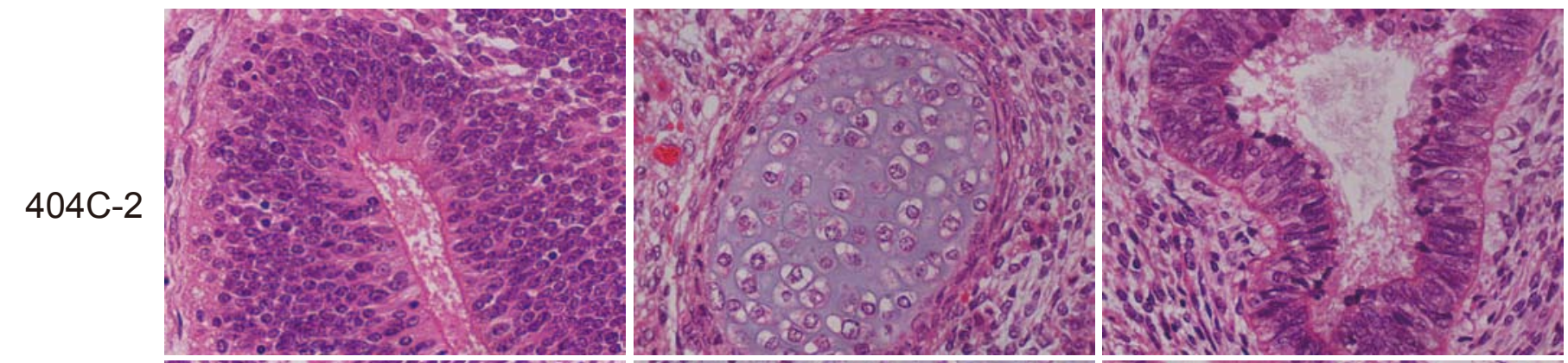

409B-2

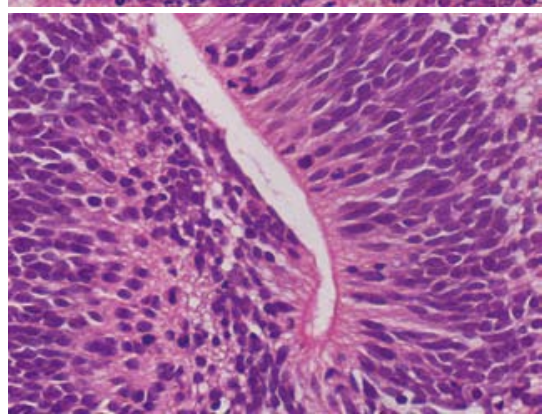

418C-1

421C-1

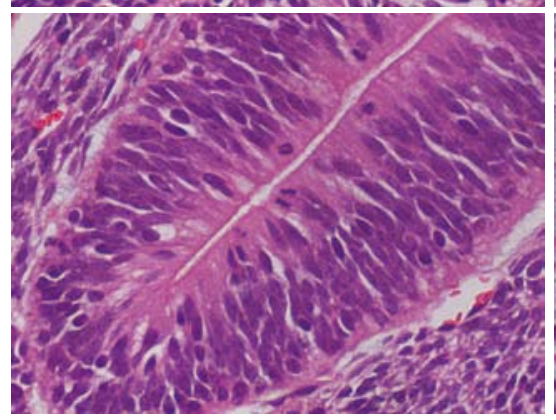

428C-2
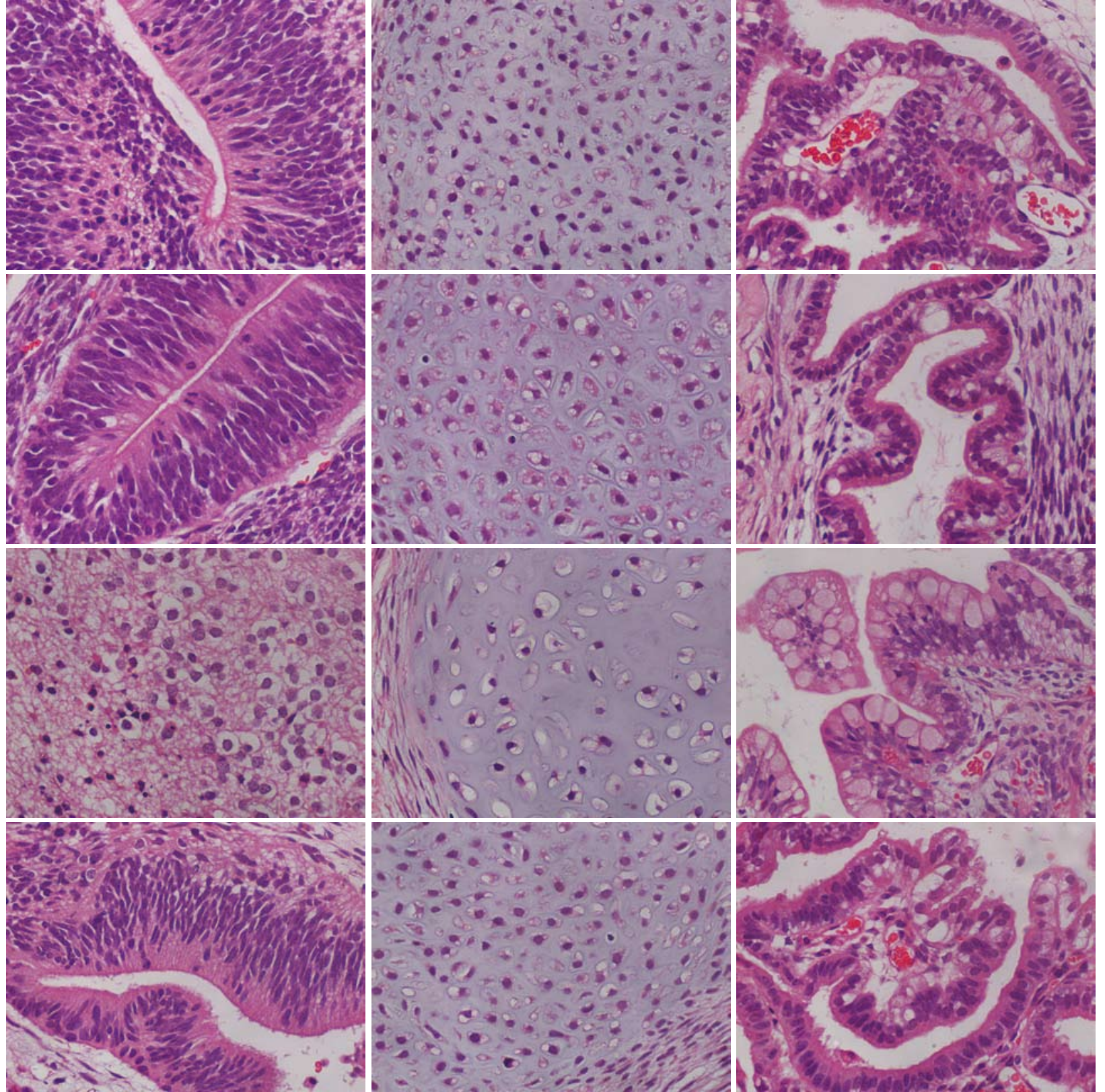

454E-2
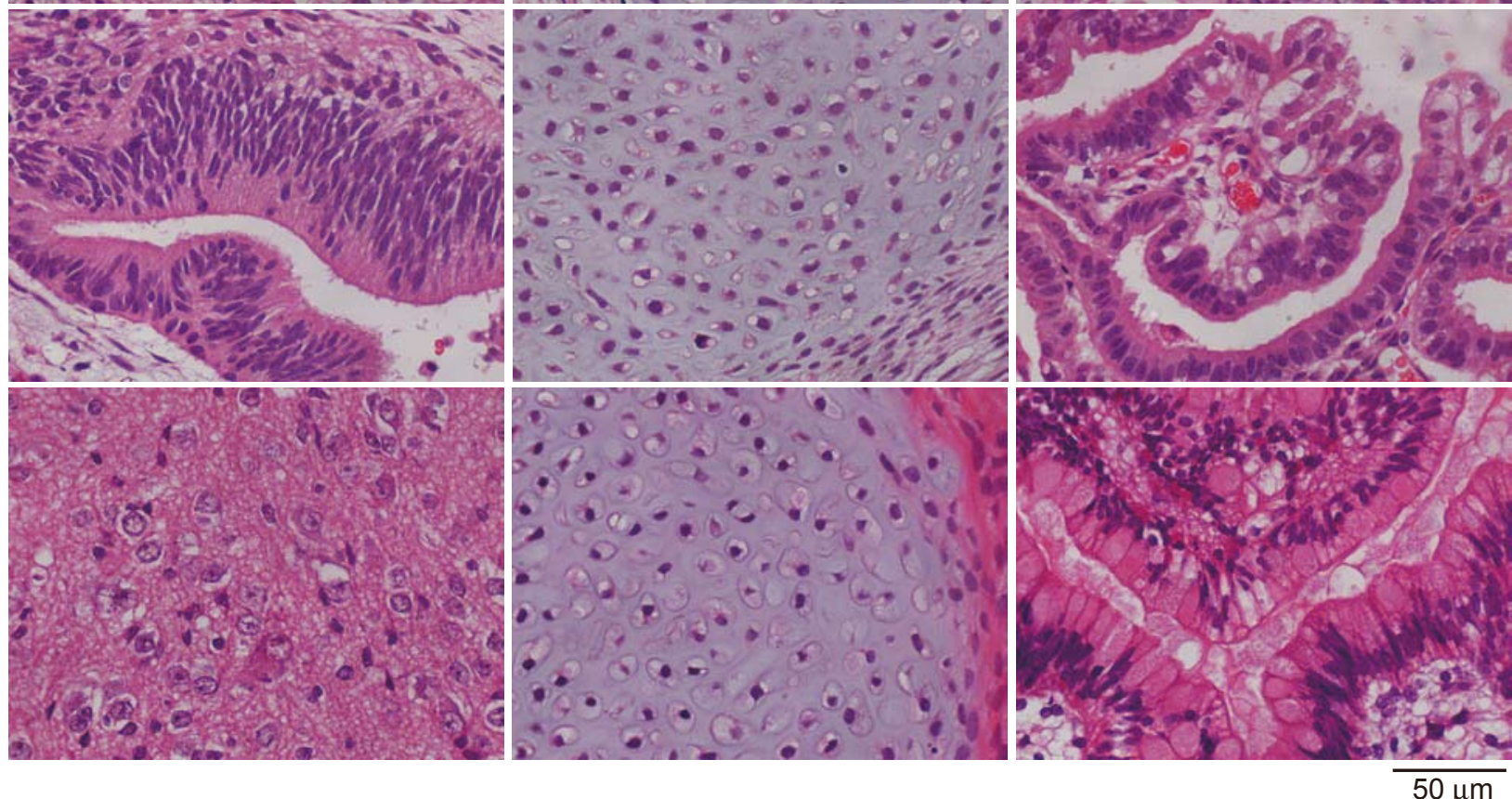

Supplementary Figure 8. Teratoma derived from pla-iPSCs.

Hematoxylin and eosin staining of teratoma derived from the indicated iPSC clones. 
a
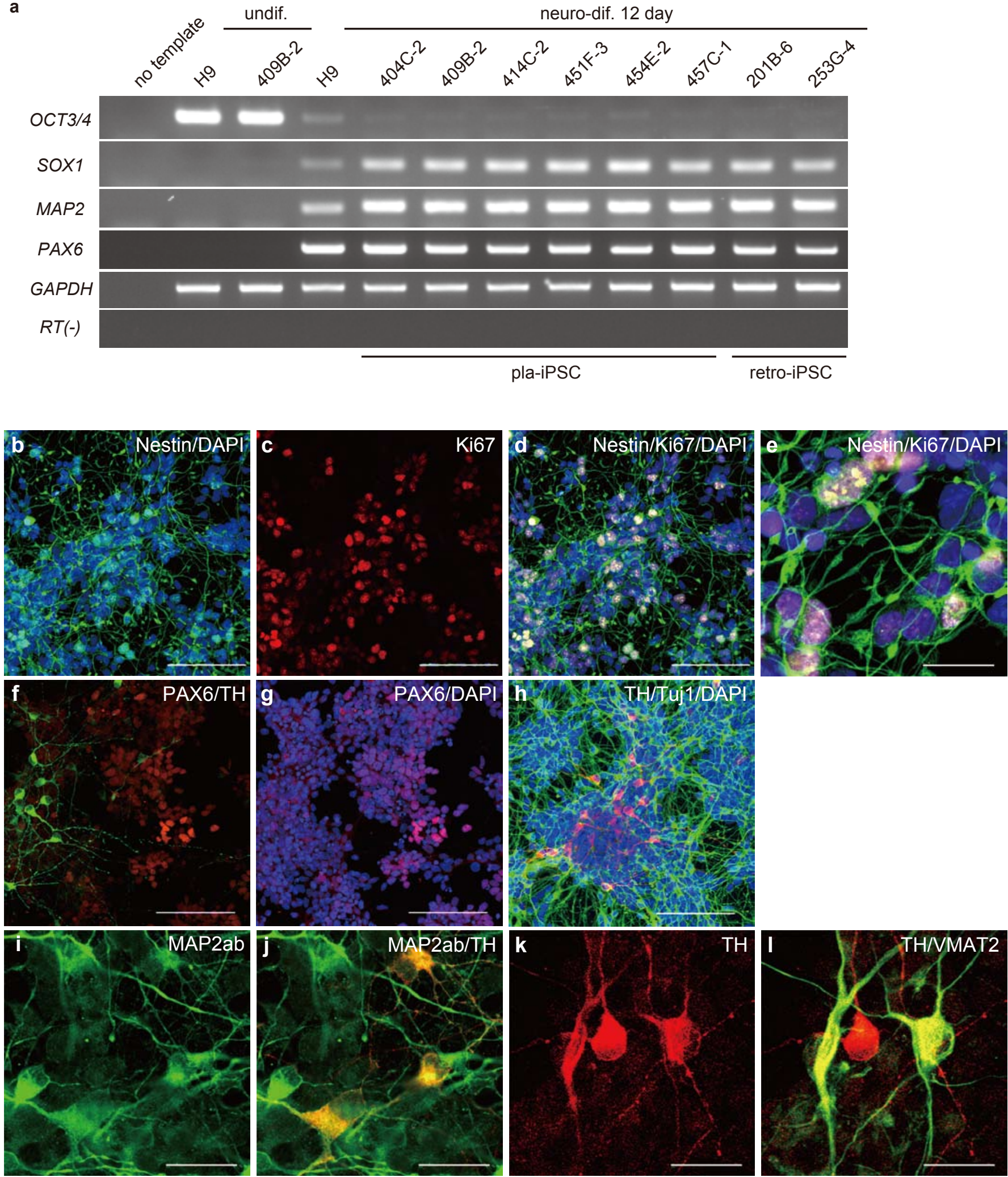

Supplementary Figure 9. Differentiation of pla-iPSCs into dopamine neurons.

(a) Expression of neural marker genes (SOX1, MAP2, and PAX6) detected by RT-PCR analyse. Total RNA was isolated from H9 ESCs, pla-iPSCs, and retro-iPSCs under undifferentiation condition (undif.) or 12 days after neural differentiation (neuro-dif.). (b-I) Twenty-nine days after induction using serum-free culture of embryoid body-like aggregates (SFEB) combined with double SMAD inhibition by a BMP antagonist and an Activin/Nodal inhibitor, the pla-iPSC clone, 454E-2, had differentiated into neurons. (b-d) Double immunostaining for Nestin (green) and Ki67 (red) with DAPI nuclear staining (blue). A high magnification image is shown in (e). ( $\mathbf{f}$ and $\mathbf{g})$ Double immunostaining for PAX6 (red) and TH (green) with DAPI nuclear staining (blue) in a same visual field. (h) Double immunostaining for TH (red) and TuJ1 (green) with DAPI. (i and $\mathbf{j}$ ) Double immunostaining for TH (red) and MAP2ab (green). ( $\mathbf{k}$ and $\mathbf{I})$ Double immunostaining for TH (Red) and the vesicular monoamine transporter 2 (VMAT2, green). Scale bars: $100 \mu \mathrm{m}$ in (b-e, f-h); $20 \mu \mathrm{m}$ in (e, i-I). 
Supplementary Table 1. Comparison of integration-free methods of human iPSC generation.

\begin{tabular}{|c|c|c|c|c|c|c|}
\hline Method & Efficiency $^{\mathrm{a}}$ & $\begin{array}{l}\text { iPSC generation } \\
\text { from adult cells }\end{array}$ & $\begin{array}{l}\text { Preparation } \\
\text { of materials }\end{array}$ & Delivery procedure & $\begin{array}{l}\text { Removal of } \\
\text { exogenous factors }\end{array}$ & References \\
\hline Adenovirus & $+\left(2 \times 10^{-6}\right)^{b}$ & Not tested & $+^{\mathrm{h}}$ & $\begin{array}{l}++ \\
\text { (2 infections) }\end{array}$ & $++^{\prime}$ & $n$ \\
\hline Sendai virus & $\begin{array}{l}+++ \\
\left(1 \times 10^{-2}\right)^{c}\end{array}$ & $\begin{array}{l}++ \\
(1 \mathrm{HDF}, 1 \mathrm{~T} \text { cell })\end{array}$ & $+^{h}$ & $\begin{array}{l}+++ \\
\quad(1 \text { infection })\end{array}$ & $+^{m}$ & $0, p$ \\
\hline Episomal plasmi & $\left(3.9 \times 10^{-4}\right)^{d}$ & $\begin{array}{l}+++ \\
(8 \mathrm{HDF}, 2 \mathrm{DP})\end{array}$ & +++ & $\begin{array}{l}+++ \\
(1 \text { transfection })\end{array}$ & $++^{1}$ & \\
\hline Minicircle DNA & $+\left(5 \times 10^{-5}\right)^{e}$ & $\begin{array}{l}++ \\
(3 \mathrm{ASC})\end{array}$ & $t^{i}$ & $+{ }^{+}(3$ transfections, FACS sorting) & ++1 & $q$ \\
\hline RNA & $\begin{array}{l}+++ \\
\left(4.4 \times 10^{-2}\right)^{f}\end{array}$ & $+(1 \mathrm{HDF})$ & $t^{\mathrm{j}}$ & $+(16$ daily transfections) & +++ & $r$ \\
\hline Protein & $+\left(2.6 \times 10^{-5}\right)^{g}$ & Not tested & $+^{k}$ & $\begin{array}{l}+ \\
(6 \text { cycle treatments })\end{array}$ & +++ & s \\
\hline
\end{tabular}

${ }^{\mathrm{a}}$ The highest figure reported in each study.

IIMR90 embryonic fibroblasts.

${ }^{\mathrm{c}} \mathrm{BJ}$ neonate fibroblasts.

${ }^{\mathrm{d}}$ Adult fibroblasts.

${ }^{\mathrm{e}}$ Adult adipose stem cells (ASC), after sorting of transfected cells.

${ }^{f}$ Fibroblasts produced by directed differentiation of H1 ESC.

${ }^{9}$ Newborn fibroblasts.

${ }^{\mathrm{h}}$ Production of virus in packaging cells.

'Construction of minicircle vectors.

${ }^{i}$ in vitro transcription with modified nucleotide.

${ }^{\mathrm{k}}$ Production of proteins in HEK293 cells.

'Rare integration events need to be ruled out.

${ }^{\mathrm{m}}$ Sendai virus tend to replicate in cytoplasm.

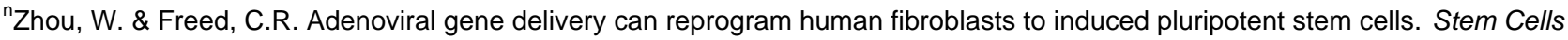
27, 2667-2674 (2009).

${ }^{\circ}$ Fusaki, N., Ban, H., Nishiyama, A., Saeki, K. \& Hasegawa, M. Efficient induction of transgene-free human pluripotent stem cells using a vector based on Sendai virus, an RNA virus that does not integrate into the host genome. Proc Jpn Acad Ser B Phys Biol Sci 85, 348-362 (2009).

${ }^{\mathrm{p}}$ Seki, T., et al. Generation of induced pluripotent stem cells from human terminally differentiated circulating T cells. Cell Stem Cell 7, 11-14 (2010).

${ }^{\mathrm{q}} \mathrm{Jia}, \mathrm{F} .$, et al. A nonviral minicircle vector for deriving human iPS cells. Nat Methods 7, 197-199 (2010).

'Warren, L., et al. Highly Efficient Reprogramming to Pluripotency and Directed Differentiation of Human Cells with Synthetic Modified mRNA. Cell Stem Cell 7, 618-30 (2010).

${ }^{\mathrm{s}} \mathrm{Kim}$, D.H., et al. Generation of Human Induced Pluripotent Stem Cells by Direct Delivery of Reprogramming Proteins. Cell Stem Cell 4, 472-476 (2009). 
Supplementary Table 2. Summary of plasmid mixtures.

\begin{tabular}{|c|c|c|c|}
\hline Mixture name & Plasmid name & Amount ( $\mu \mathrm{g})$ & Genes \\
\hline \multirow[t]{3}{*}{$\mathrm{Y} 1$} & pCXLE-hOCT3/4 & 1 & $0 C T 3 / 4$ \\
\hline & pCXLE-hSK & 1 & SOX2, KLF4 \\
\hline & $\mathrm{pCXLE}-\mathrm{hMLN}$ & & $c-M Y C, \angle I N 28, N A N O G$ \\
\hline \multirow[t]{3}{*}{ Y2 } & pCXLE-hOCT3/4-shp53 & 1 & OCT3/4, p53 shRNA \\
\hline & pCXLE-hSK & 1 & SOX2, KLF4 \\
\hline & pCXLE-hMLN & 1 & $c-M Y C, \angle I N 28, N A N O G$ \\
\hline \multirow[t]{3}{*}{ Y3 } & pCXLE-hOCT3/4 & 1 & OCT3/4 \\
\hline & pCXLE-hSK & 1 & SOX2, KLF4 \\
\hline & $\mathrm{pCXLE}-\mathrm{hUL}$ & & $\angle-M Y C, \angle I N 28$ \\
\hline \multirow[t]{3}{*}{$\overline{Y 4}$} & pCXLE-hOCT3/4-shp53 & 1 & OCT3/4,p53 shRNA \\
\hline & pCXLE-hSK & 1 & SOX2, KLF4 \\
\hline & pCXLE-hUL & 1 & $\angle-M Y C, L I N 28$ \\
\hline \multirow[t]{3}{*}{ T1 } & pEP4EO2SET2K & 1.125 & OCT3/4, SOX2, SV4OLT, KLF4 \\
\hline & pEP4EO2SEN2K & 1.125 & OCT3/4, SOX2, NANOG, KLF4 \\
\hline & pCEP4-M2L & 0.75 & $c-M Y C, L I N 28$ \\
\hline \multirow[t]{2}{*}{ T2 } & pEP4EO2SET2K & 0.91 & $O C T 3 / 4, S O X 2, S V 4 O L T, K L F 4$ \\
\hline & pEP4EO2SCK2MEN2L & 2.09 & OCT3/4, SOX2, KLF4, c-MYC, NANOG, LIN28 \\
\hline \multirow[t]{3}{*}{ T3 } & pEP4EO2SET2K & 0.8 & OCT3/4, SOX2, SV $40 L T, K L F 4$ \\
\hline & pEP4EO2SEN2L & 1.05 & $O C T 3 / 4, S O X 2, N A N O G, L I N 28$ \\
\hline & pEP4EO2SEM2K & 1.15 & $O C T 3 / 4, S O X 2, c-M Y C, K L F 4$ \\
\hline
\end{tabular}


Supplementary Table 3. Summary of the iPSC induction experiments.

\begin{tabular}{|c|c|c|c|c|c|c|}
\hline \multirow{2}{*}{\begin{tabular}{|c|} 
Parental \\
cells
\end{tabular}} & \multirow[b]{2}{*}{ Exp. ID } & \multirow[b]{2}{*}{ Factors } & \multicolumn{2}{|c|}{ MEF feeder } & \multicolumn{2}{|c|}{ SNL feeder } \\
\hline & & & $\begin{array}{c}\text { non } \\
\text { ES-like }\end{array}$ & ES-like & $\begin{array}{c}\text { non } \\
\text { ES-like }\end{array}$ & ES-like \\
\hline \multirow[t]{7}{*}{ HDF1419 } & \multirow{5}{*}{438} & Y1 & 1 & 0 & 0 & 1 \\
\hline & & Y2 & 24 & 17 & 5 & 17 \\
\hline & & Y3 & 18 & 8 & 5 & 11 \\
\hline & & $\mathrm{Y4}$ & 81 & 60 & 66 & 108 \\
\hline & & control & 0 & 0 & 0 & 0 \\
\hline & \multirow{2}{*}{449} & Y4 & 47 & 134 & 43 & 150 \\
\hline & & control & 0 & 0 & 0 & 0 \\
\hline \multirow[t]{5}{*}{ TIG121 } & \multirow[t]{5}{*}{380} & $\mathrm{Y} 1$ & 2 & 3 & 0 & 0 \\
\hline & & $\mathrm{Y} 2$ & 11 & 10 & 1 & 4 \\
\hline & & $\mathrm{Y3}$ & 9 & 23 & 0 & 0 \\
\hline & & Y4 & 24 & 97 & 5 & 11 \\
\hline & & control & 0 & 0 & 0 & 0 \\
\hline \multirow{34}{*}{ TIG120 } & \multirow{5}{*}{380} & Y1 & 1 & 3 & 0 & 0 \\
\hline & & Y2 & 7 & 11 & 1 & 2 \\
\hline & & Y3 & 4 & 16 & 0 & 4 \\
\hline & & Y4 & 37 & 71 & 17 & 25 \\
\hline & & control & 0 & 0 & 0 & 0 \\
\hline & \multirow{5}{*}{428} & Y1 & 0 & 1 & 0 & 0 \\
\hline & & $\mathrm{Y} 2$ & 5 & 10 & 3 & 0 \\
\hline & & Y3 & - & - & 2 & 1 \\
\hline & & Y4 & 20 & 61 & 3 & 15 \\
\hline & & control & 0 & 0 & 0 & 0 \\
\hline & \multirow[t]{8}{*}{448} & Y1 & 0 & 3 & 0 & 0 \\
\hline & & $\mathrm{Y} 2$ & 2 & 12 & 0 & 4 \\
\hline & & Y3 & 4 & 30 & 1 & 1 \\
\hline & & Y4 & 23 & 92 & 6 & 19 \\
\hline & & T1 & 7 & 0 & 14 & 0 \\
\hline & & $\mathrm{T} 2$ & 8 & 0 & 1 & 0 \\
\hline & & T3 & 20 & 0 & 5 & 0 \\
\hline & & control & 0 & 0 & 0 & 0 \\
\hline & \multirow[t]{8}{*}{455} & Y1 & 3 & 5 & 0 & 0 \\
\hline & & Y2 & 5 & 24 & 1 & 8 \\
\hline & & Y3 & 9 & 45 & 2 & 9 \\
\hline & & Y4 & 37 & 154 & 10 & 67 \\
\hline & & T1 & 15 & 0 & 8 & 0 \\
\hline & & T2 & 11 & 0 & 1 & 1 \\
\hline & & T3 & 48 & 0 & 6 & 1 \\
\hline & & control & 0 & 0 & 0 & 0 \\
\hline & \multirow[t]{8}{*}{522} & $\mathrm{Y} 1$ & 1 & 1 & 0 & 0 \\
\hline & & $\mathrm{Y} 2$ & 4 & 4 & 2 & 2 \\
\hline & & Y3 & 2 & 0 & 0 & 0 \\
\hline & & Y4 & 5 & 16 & 2 & 3 \\
\hline & & T1 & 4 & 0 & 0 & 0 \\
\hline & & T2 & 1 & 0 & 0 & 0 \\
\hline & & T3 & 6 & 0 & 0 & 0 \\
\hline & & control & 0 & 0 & 0 & 0 \\
\hline \multirow[t]{4}{*}{ HDF1388 } & \multirow[t]{4}{*}{404} & Y1 & 0 & 0 & 0 & 0 \\
\hline & & $\mathrm{Y} 2$ & 0 & 1 & 0 & 0 \\
\hline & & Y3 & 1 & 2 & 0 & 0 \\
\hline & & $Y 4$ & 1 & 5 & 0 & 2 \\
\hline
\end{tabular}




\begin{tabular}{|c|c|c|c|c|c|c|}
\hline & & control & 0 & 0 & $\overline{0}$ & 0 \\
\hline & 409 & $\mathrm{Y} 1$ & 0 & 0 & 0 & 0 \\
\hline & & $\overline{Y 2}$ & 0 & $\overline{0}$ & $\overline{0}$ & 0 \\
\hline & & Y3 & 0 & 2 & 0 & 0 \\
\hline & & $\overline{Y 4}$ & 2 & 12 & $\overline{0}$ & 1 \\
\hline & & T1 & 9 & 0 & 5 & 0 \\
\hline & & T2 & 14 & 4 & 3 & 5 \\
\hline & & T3 & 56 & 2 & 36 & 1 \\
\hline & & $\overline{\text { control }}$ & 0 & 0 & 0 & 0 \\
\hline & 414 & $\mathrm{Y1}$ & 0 & 0 & 0 & 0 \\
\hline & & $\overline{Y 2}$ & 4 & 1 & 1 & 0 \\
\hline & & Y3 & 0 & 1 & 0 & 0 \\
\hline & & Y4 & 3 & 9 & 0 & 2 \\
\hline & & T1 & 5 & $\overline{0}$ & 3 & $\overline{0}$ \\
\hline & & T2 & 30 & 0 & 1 & 6 \\
\hline & & T3 & 18 & 0 & 5 & 0 \\
\hline & & control & 0 & 0 & 0 & 0 \\
\hline & 415 & Y1 & 0 & 0 & 0 & 0 \\
\hline & & $\mathrm{Y} 2$ & 0 & 0 & 1 & 0 \\
\hline & & $\overline{Y 3}$ & 0 & 0 & 0 & 0 \\
\hline & & $\overline{Y 4}$ & 1 & 5 & 2 & 1 \\
\hline & & T1 & 13 & 0 & 3 & 0 \\
\hline & & $\bar{T} 2$ & 28 & 0 & 0 & 2 \\
\hline & & T3 & 26 & 0 & 12 & 0 \\
\hline & & control & 0 & 0 & 0 & 0 \\
\hline & 417 & $\mathrm{Y} 1$ & 0 & 0 & 0 & 0 \\
\hline & & Y2 & 103 & 0 & 0 & 0 \\
\hline & & Y3 & 0 & 1 & 0 & 0 \\
\hline & & $\overline{Y 4}$ & 1 & 4 & 0 & 3 \\
\hline & & control & 0 & 0 & 0 & 0 \\
\hline TIG114 & 418 & $\mathrm{Y} 1$ & 0 & 0 & 0 & 0 \\
\hline & & $\mathrm{Y} 2$ & 1 & 0 & 1 & 0 \\
\hline & & $\mathrm{Y3}$ & 2 & 0 & 0 & 0 \\
\hline & & Y4 & 12 & 4 & 0 & 1 \\
\hline & & \begin{tabular}{|c|} 
control \\
\end{tabular} & 0 & 0 & 0 & 0 \\
\hline HDF1377 & 436 & $\mathrm{Y} 1$ & 3 & 1 & 0 & 0 \\
\hline & & $\mathrm{Y} 2$ & 78 & 2 & 3 & 1 \\
\hline & & Y3 & 7 & 8 & 1 & 1 \\
\hline & & $\overline{Y 4}$ & 27 & 26 & 8 & 11 \\
\hline & & T2 & 16 & 0 & 2 & 0 \\
\hline & & control & 0 & 0 & 0 & 0 \\
\hline HDF1429 & 437 & Y4 & 34 & 50 & 4 & 24 \\
\hline & & $\mathrm{T} 2$ & 22 & 0 & 1 & 0 \\
\hline & & control & 0 & 0 & 0 & 0 \\
\hline HDF1437 & $427^{a}$ & $\mathrm{Y} 1$ & 5 & 0 & 0 & 1 \\
\hline & & $\overline{Y 2}$ & 9 & 1 & 0 & 1 \\
\hline & & Y3 & 11 & 2 & 3 & 5 \\
\hline & & Y4 & 27 & 4 & 2 & 12 \\
\hline & & control & 0 & 0 & 0 & 0 \\
\hline & 460 & Y1 & 0 & 0 & 0 & 0 \\
\hline & & $\overline{Y 2}$ & 6 & 0 & 1 & 3 \\
\hline & & $\overline{Y 3}$ & 15 & 2 & 0 & 1 \\
\hline & & Y4 & 43 & 11 & 19 & 29 \\
\hline & & $\overline{T 1}$ & 9 & 0 & 10 & 0 \\
\hline
\end{tabular}




\begin{tabular}{|c|c|c|c|c|c|c|}
\hline & & & & & & \\
\hline & & $\mathrm{T} 2$ & 18 & 0 & 8 & 1 \\
\hline & & T3 & 25 & 0 & 19 & 0 \\
\hline & & control & 0 & 0 & 0 & 0 \\
\hline & \multirow[t]{8}{*}{466} & Y1 & 0 & 0 & 0 & 0 \\
\hline & & $\mathrm{Y} 2$ & 21 & 0 & 4 & 13 \\
\hline & & $\mathrm{Y3}$ & 22 & 0 & 0 & 8 \\
\hline & & $\mathrm{Y4}$ & 174 & 44 & 81 & 100 \\
\hline & & $\overline{T 1}$ & 13 & 0 & 8 & 0 \\
\hline & & $\mathrm{T} 2$ & 10 & 0 & 3 & 0 \\
\hline & & T3 & 38 & 0 & 40 & 0 \\
\hline & & control & 0 & 0 & 0 & 0 \\
\hline & \multirow{8}{*}{521} & Y1 & 0 & 0 & 0 & 0 \\
\hline & & $\mathrm{Y} 2$ & 5 & 1 & 0 & 3 \\
\hline & & $\mathrm{Y3}$ & 1 & 0 & 0 & 0 \\
\hline & & Y4 & 17 & 5 & 6 & 11 \\
\hline & & $\mathrm{T} 1$ & 14 & 0 & 2 & 0 \\
\hline & & $\mathrm{T} 2$ & 5 & 0 & 3 & 0 \\
\hline & & T3 & 32 & 0 & 19 & 0 \\
\hline & & control & 0 & 0 & 0 & 0 \\
\hline \multirow[t]{15}{*}{ HDF1554 } & \multirow[t]{5}{*}{426} & $\mathrm{Y} 1$ & 0 & 0 & 0 & 0 \\
\hline & & $\mathrm{Y} 2$ & 1 & 0 & 0 & 0 \\
\hline & & $\mathrm{Y3}$ & 2 & 0 & 0 & 0 \\
\hline & & $\overline{Y 4}$ & 6 & 0 & 1 & 2 \\
\hline & & control & 0 & 0 & 0 & 0 \\
\hline & \multirow[t]{5}{*}{465} & $\mathrm{Y} 1$ & 0 & 0 & 0 & 1 \\
\hline & & $\mathrm{Y} 2$ & 6 & 0 & 1 & 2 \\
\hline & & $\overline{Y 3}$ & 4 & 0 & 0 & 0 \\
\hline & & Y4 & 35 & 0 & 8 & 22 \\
\hline & & control & 0 & 0 & 0 & 0 \\
\hline & \multirow[t]{5}{*}{468} & Y1 & 0 & 0 & 0 & 0 \\
\hline & & $\mathrm{Y} 2$ & 7 & 0 & 1 & 2 \\
\hline & & $\mathrm{Y3}$ & 2 & 0 & 1 & 2 \\
\hline & & Y4 & 11 & 5 & 11 & 25 \\
\hline & & control & 0 & 0 & 0 & 0 \\
\hline \multirow{11}{*}{ TIG107 } & \multirow{5}{*}{421} & Y1 & 1 & 0 & - & - \\
\hline & & $\mathrm{Y} 2$ & 9 & 0 & - & - \\
\hline & & $\mathrm{Y3}$ & 0 & 0 & - & - \\
\hline & & $\overline{Y 4}$ & 3 & 4 & - & - \\
\hline & & control & 0 & 0 & - & - \\
\hline & \multirow[t]{6}{*}{478} & $\mathrm{Y} 1$ & 0 & 0 & 0 & 0 \\
\hline & & $\mathrm{Y} 2$ & 0 & 0 & 0 & 0 \\
\hline & & Y3 & 0 & 0 & 0 & 0 \\
\hline & & $\mathrm{Y4}$ & 2 & 5 & 0 & 0 \\
\hline & & $\overline{Y 4}$ & 0 & 2 & 0 & 0 \\
\hline & & control & 0 & 0 & 0 & 0 \\
\hline \multirow[t]{9}{*}{ DP74 } & \multirow[t]{7}{*}{451} & $\mathrm{Y} 1$ & 0 & 0 & 0 & 0 \\
\hline & & $\mathrm{Y} 2$ & 5 & 8 & 3 & 13 \\
\hline & & Y3 & 2 & 5 & 3 & 7 \\
\hline & & $\mathrm{Y4}$ & 7 & 27 & 10 & 83 \\
\hline & & $\mathrm{T} 2$ & 1 & 0 & 1 & 4 \\
\hline & & T3 & 0 & 0 & 3 & 0 \\
\hline & & control & 0 & 0 & 0 & 0 \\
\hline & \multirow[t]{2}{*}{454} & $\mathrm{Y} 1$ & 0 & 5 & 0 & 3 \\
\hline & & Y2 & 8 & 12 & 2 & 11 \\
\hline
\end{tabular}




\begin{tabular}{|c|c|c|c|c|c|c|}
\hline & & Y3 & 4 & 8 & 2 & 11 \\
\hline & & $\mathrm{Y4}$ & 10 & 20 & 10 & 27 \\
\hline & & T1 & 0 & 0 & 0 & 0 \\
\hline & & T2 & 0 & 0 & 1 & 4 \\
\hline & & T3 & 0 & 0 & 0 & 1 \\
\hline & & control & 0 & 0 & 0 & 0 \\
\hline & 457 & Y1 & 1 & 0 & 0 & 0 \\
\hline & & Y2 & 2 & 0 & 0 & 3 \\
\hline & & Y3 & 2 & 2 & 0 & 1 \\
\hline & & Y4 & 2 & 7 & 2 & 13 \\
\hline & & T1 & 0 & 0 & 0 & 0 \\
\hline & & T2 & 0 & 0 & 2 & 0 \\
\hline & & T3 & 1 & 0 & 6 & 0 \\
\hline & & control & 0 & 0 & 0 & 0 \\
\hline & 469 & $\mathrm{Y} 1$ & 2 & 0 & 1 & 1 \\
\hline & & Y2 & 4 & 6 & 4 & 14 \\
\hline & & Y3 & 8 & 11 & 4 & 17 \\
\hline & & $\overline{Y 4}$ & 28 & 23 & 18 & 97 \\
\hline & & T1 & 0 & 0 & 0 & 0 \\
\hline & & T2 & 2 & 0 & 1 & 2 \\
\hline & & T3 & 0 & 0 & 4 & 4 \\
\hline & & control & 0 & 0 & 0 & 0 \\
\hline DP94 & 446 & $\mathrm{Y1}$ & 2 & 2 & 5 & 5 \\
\hline & & Y2 & 17 & 4 & 19 & 21 \\
\hline & & $\overline{Y 3}$ & 14 & 3 & 11 & 7 \\
\hline & & Y4 & 27 & 10 & 31 & 30 \\
\hline & & $\mathrm{T1}$ & 0 & 0 & 3 & 1 \\
\hline & & T2 & 0 & 0 & 0 & 0 \\
\hline & & T3 & 0 & 0 & 0 & 0 \\
\hline & & control & 0 & 0 & 0 & 0 \\
\hline & 450 & $\mathrm{Y1}$ & 5 & 0 & 2 & 0 \\
\hline & & Y2 & 8 & 0 & 11 & 19 \\
\hline & & Y3 & 9 & 2 & 10 & 8 \\
\hline & & Y4 & 24 & 2 & 21 & 35 \\
\hline & & T1 & 0 & 0 & 2 & 1 \\
\hline & & T2 & 0 & 0 & 0 & 2 \\
\hline & & T3 & 0 & 0 & 3 & 1 \\
\hline & & control & 0 & 0 & 0 & 0 \\
\hline & 453 & Y1 & 5 & 1 & 1 & 3 \\
\hline & & $\mathrm{Y} 2$ & 12 & 3 & 9 & 12 \\
\hline & & Y3 & 9 & 5 & 7 & 7 \\
\hline & & Y4 & 36 & 22 & 30 & 24 \\
\hline & & T1 & 0 & 0 & 1 & 0 \\
\hline & & $\mathrm{T} 2$ & 1 & 0 & 0 & 0 \\
\hline & & T3 & 0 & 0 & 0 & 0 \\
\hline & & control & 0 & 0 & 0 & 0 \\
\hline
\end{tabular}

${ }^{a}$ In the exp. 427, only $0.8 \times 10^{5}$ cells were plated onto SNL feeder cells.

${ }^{*}$ Exp. ID 448, 455, 522, 409, 414, 415, 460, 466, 521, 454, 457, 469, 446, 450, and 453 were used for Fig. 1d. 
Supplementary Table 4. Karyotype of episomal vector-derived iPSCs.

\begin{tabular}{|c|c|c|c|c|c|c|c|}
\hline Clone & passage & Karyotype & origin & Age & $\operatorname{Sex}^{2}$ & Race & actors \\
\hline & & & fibroblasts & & & & \\
\hline 409B-2 & p19 & $46, X X[20]$ & HDF1388 & 36 & $\mathrm{~F}$ & C & Y4 \\
\hline 414C-2 & p19 & $46, X X[20]$ & HDF1388 & 36 & $\mathrm{~F}$ & C & Y4 \\
\hline $418 C-1$ & p19 & $46, X Y[20]$ & TIG114 & 36 & $M$ & J & Y4 \\
\hline $421 C-1$ & p19 & $46, X X[20]$ & TIG107 & 81 & $\mathrm{~F}$ & $\mathrm{~J}$ & Y4 \\
\hline $426 C-2$ & p14 & $46, X X[20]$ & HDF1554 & 77 & $\mathrm{~F}$ & C & Y4 \\
\hline 427D-4 & p13 & $46, \mathrm{XY}, \operatorname{add}(3)(\mathrm{q} 13.2)[20]$ & HDF1437 & 56 & $M$ & C & Y4 \\
\hline \multirow[t]{2}{*}{$428 C-2$} & p20 & $46, X X[20]$ & TIG120 & 6 & $\mathrm{~F}$ & $\mathrm{~J}$ & Y4 \\
\hline & & & DP cells & & & & \\
\hline $451 \mathrm{~F}-3$ & p9 & $46, X X[20]$ & DP74 & 16 & $\mathrm{~F}$ & $\mathrm{~J}$ & Y4 \\
\hline $453 F-2$ & p12 & $46, X X[20]$ & DP94 & 16 & $\mathrm{~F}$ & $J$ & Y4 \\
\hline 454B-1 & p12 & $46, X X[20]$ & DP74 & 16 & $\mathrm{~F}$ & $\mathrm{~J}$ & Y1 \\
\hline $454 C-2$ & p10 & $46, X X[20]$ & DP74 & 16 & $\mathrm{~F}$ & $\mathrm{~J}$ & Y2 \\
\hline 454D-1 & $\mathrm{p} 11$ & $46, X X[20]$ & DP74 & 16 & $\mathrm{~F}$ & $\mathrm{~J}$ & Y3 \\
\hline $454 \mathrm{E}-2$ & p12 & $46, X X[20]$ & DP74 & 16 & $\mathrm{~F}$ & $J$ & Y4 \\
\hline $454 \mathrm{~F}-1$ & $\mathrm{p} 13$ & $46, X X, \operatorname{add}(17)(q 25)[15] / 46, X X[5]$ & DP74 & 16 & $\mathrm{~F}$ & $\mathrm{~J}$ & T2 \\
\hline $457 \mathrm{C}-1$ & p12 & $46, X X[20]$ & DP74 & 16 & $\mathrm{~F}$ & $\mathrm{~J}$ & Y4 \\
\hline
\end{tabular}

${ }^{\mathrm{a}} \mathrm{M}$, male; $F$, female

${ }^{\mathrm{b}} \mathrm{J}$, Japanese; C, Caucasian. 
SupplementaryTable 5. STR analyses of pla-iPSCs.

\begin{tabular}{|c|c|c|c|c|c|c|c|c|c|c|c|c|}
\hline Locus/clone & \multicolumn{2}{|c|}{ HDF1388 } & \multicolumn{2}{|c|}{ 409B-2 } & \multicolumn{2}{|c|}{ 414C-2 } & \multicolumn{2}{|c|}{ DP74 } & \multicolumn{2}{|c|}{$454 \mathrm{E}-2$} & \multicolumn{2}{|c|}{$457 C-1$} \\
\hline D3S1358 & 16 & 18 & 16 & 18 & 16 & 18 & 17 & & 17 & & 17 & \\
\hline THO1 & 6 & & 6 & & 6 & & 7 & & 7 & & 7 & \\
\hline D21S11 & 29 & & 29 & & 29 & & 29 & 30 & 29 & 30 & 29 & 30 \\
\hline D18S51 & 15 & & 15 & & 15 & & 13 & 19 & 13 & 19 & 13 & 19 \\
\hline Penta_E & 7 & 19 & 7 & 19 & 7 & 19 & 5 & 15 & 5 & 15 & 5 & 15 \\
\hline D5S818 & 12 & & 12 & & 12 & & 12 & 13 & 12 & 13 & 12 & 13 \\
\hline D13S317 & 9 & 13 & 9 & 13 & 9 & 13 & 10 & 14 & 10 & 14 & 10 & 14 \\
\hline D7S820 & 9 & 10 & 9 & 10 & 9 & 10 & 11 & & 11 & & 11 & \\
\hline D16S539 & 11 & 13 & 11 & 13 & 11 & 13 & 9 & 10 & 9 & 10 & 9 & 10 \\
\hline CSF1PO & 11 & & 11 & & 11 & & 12 & 14 & 12 & 14 & 12 & 14 \\
\hline Penta_D & 9 & 11 & 9 & 11 & 9 & 11 & 10 & 13 & 10 & 13 & 10 & 13 \\
\hline$A M E L$ & $X$ & & $X$ & & $x$ & & $X$ & & $X$ & & $X$ & \\
\hline$v W A$ & 15 & 18 & 15 & 18 & 15 & 18 & 17 & 21 & 17 & 21 & 17 & 21 \\
\hline D8S1179 & 8 & 10 & 8 & 10 & 8 & 10 & 11 & 12 & 11 & 12 & 11 & 12 \\
\hline TPOX & 8 & 9 & 8 & 9 & 8 & 9 & 8 & & 8 & & 8 & \\
\hline$F G A$ & 20 & 22 & 20 & 22 & 20 & 22 & 21 & 24 & 21 & 24 & 21 & 24 \\
\hline
\end{tabular}


Supplementary Table 6. Pearson correlation coefficient table.

\begin{tabular}{|c|c|c|c|c|c|c|c|c|c|c|c|c|c|c|c|c|c|}
\hline Cells & $404 C-2$ & 409B-2 & $414 C-2$ & $451 \mathrm{~F}-3$ & 454B-1 & $454 \mathrm{E}-2$ & $457 \mathrm{C}-1$ & 201B-6 & 201B-7 & $253 G-4$ & $\mathrm{H} 1$ & $\mathrm{H} 9$ & KhES1 & KhES3 & HDF1388 & DP74 & CellType \\
\hline $404 C-2$ & 1 & 0.99134 & 0.99538 & 0.99044 & 0.99031 & 0.99198 & 0.98828 & 0.99044 & 0.97643 & 0.99336 & 0.99492 & 0.97962 & 0.99175 & 0.98902 & 0.82664 & 0.85313 & Pla-iPSCs \\
\hline 409B-2 & 0.99134 & 1 & 0.99169 & 0.99285 & 0.99592 & 0.99557 & 0.99460 & 0.98239 & 0.98645 & 0.98373 & 0.99082 & 0.99221 & 0.98944 & 0.99396 & 0.82676 & 0.85663 & Pla-iPSCs \\
\hline $414 C-2$ & 0.99538 & 0.99169 & 1 & 0.99241 & 0.99252 & 0.99232 & 0.99139 & 0.99200 & 0.97867 & 0.99466 & 0.99332 & 0.98108 & 0.99549 & 0.98914 & 0.83475 & 0.86055 & Pla-iPSCs \\
\hline $451 \mathrm{~F}-3$ & 0.99044 & 0.99285 & 0.99241 & 1 & 0.99668 & 0.99784 & 0.99786 & 0.98416 & 0.98480 & 0.98417 & 0.99305 & 0.98925 & 0.99432 & 0.99540 & 0.83299 & 0.86326 & Pla-iPSCs \\
\hline $454 \mathrm{~B}-1$ & 0.99031 & 0.99592 & 0.99252 & 0.99668 & 1 & 0.99840 & 0.99856 & 0.98459 & 0.98912 & 0.98475 & 0.99225 & 0.99269 & 0.99324 & 0.99471 & 0.83082 & 0.86259 & Pla-iPSCs \\
\hline $454 \mathrm{E}-2$ & 0.99198 & 0.99557 & 0.99232 & 0.99784 & 0.99840 & 1 & 0.99809 & 0.98424 & 0.98611 & 0.98469 & 0.99286 & 0.99199 & 0.99286 & 0.99596 & 0.83134 & 0.86163 & Pla-iPSCs \\
\hline $457 \mathrm{C}-1$ & 0.98828 & 0.99460 & 0.99139 & 0.99786 & 0.99856 & 0.99809 & 1 & 0.98275 & 0.98878 & 0.98256 & 0.99213 & 0.99286 & 0.99335 & 0.99577 & 0.83051 & 0.86185 & Pla-iPSCs \\
\hline 201B-6 & 0.99044 & 0.98239 & 0.99200 & 0.98416 & 0.98459 & 0.98424 & 0.98275 & 1 & 0.97506 & 0.99221 & 0.98866 & 0.97083 & 0.99045 & 0.97956 & 0.82601 & 0.85383 & Retro-iPSCs \\
\hline 201B-7 & 0.97643 & 0.98645 & 0.97867 & 0.98480 & 0.98912 & 0.98611 & 0.98878 & 0.97506 & 1 & 0.97174 & 0.98394 & 0.98785 & 0.98483 & 0.98621 & 0.81791 & 0.85376 & Retro-iPSCs \\
\hline $253 G-4$ & 0.99336 & 0.98373 & 0.99466 & 0.98417 & 0.98475 & 0.98469 & 0.98256 & 0.99221 & 0.97174 & 1 & 0.99009 & 0.97106 & 0.99112 & 0.98138 & 0.82652 & 0.85200 & Retro-iPSCs \\
\hline $\mathrm{H} 1$ & 0.99492 & 0.99082 & 0.99332 & 0.99305 & 0.99225 & 0.99286 & 0.99213 & 0.98866 & 0.98394 & 0.99009 & 1 & 0.98488 & 0.99498 & 9393 & 0.82599 & 0.85782 & ESCs \\
\hline $\mathrm{H} 9$ & 0.97962 & 0.99221 & 0.98108 & 0.98925 & 0.99269 & 0.99199 & 0.99286 & 0.97083 & 0.98785 & 0.97106 & 0.98488 & 1 & 0.98325 & 0.99272 & 0.81722 & 0.85115 & ESCs \\
\hline KhES1 & 0.99175 & 0.98944 & 0.99549 & 0.99432 & 0.99324 & 0.99286 & 0.99335 & 0.99045 & 0.98483 & 0.99112 & 0.99498 & 0.98325 & 1 & 0.99191 & 0.83019 & 0.85962 & ESCs \\
\hline KhES3 & 0.98902 & 0.99396 & 0.98914 & 0.99540 & 0.99471 & 0.99596 & 0.99577 & 0.97956 & 0.98621 & 0.98138 & 0.99393 & 0.99272 & 0.99191 & 1 & 0.82553 & 0.85750 & ESCs \\
\hline HDF1388 & 0.82664 & 0.82676 & 0.83475 & 0.83299 & 0.83082 & 0.83134 & 0.83051 & 0.82601 & 0.81791 & 0.82652 & 0.82599 & 0.81722 & 0.83019 & 0.82553 & 1 & 0.96628 & Fibroblasts \\
\hline DP74 & 0.85313 & 0.85663 & 0.86055 & 0.86326 & 0.86259 & 0.86163 & 0.86185 & 0.85383 & 0.85376 & 0.85200 & 0.85782 & 0.85115 & 0.85962 & 0.85750 & 0.96628 & & DP cells \\
\hline
\end{tabular}


Supplementary Table 7. HLA Type of 107 Individuals.

\begin{tabular}{|c|c|c|c|c|c|c|c|c|}
\hline No. & Cell line & \multicolumn{2}{|c|}{ HLA-A } & \multicolumn{2}{|c|}{ HLA-B } & \multicolumn{2}{|c|}{ HLA-DRB1 } & \multirow[t]{2}{*}{ Match } \\
\hline 1 & DP1 & ${ }^{*} 24$ & *33 & ${ }^{*} 40$ & $* 44$ & ${ }^{*} 04$ & *13 & \\
\hline 2 & DP4 & ${ }^{*} 03$ & ${ }^{*} 24$ & ${ }^{*} 07$ & $* 13$ & ${ }^{*} 01$ & ${ }^{*} 07$ & \\
\hline 3 & DP6 & ${ }^{*} 02$ & ${ }^{*} 02$ & *35 & $* 40$ & ${ }^{*} 04$ & ${ }^{*} 09$ & \\
\hline 4 & DP7 & ${ }^{*} 03$ & ${ }^{*} 24$ & *44 & $* 52$ & *09 & $* 15$ & DP74 \\
\hline 5 & DP10 & *24 & ${ }^{*} 26$ & $* 13$ & $* 52$ & $* 12$ & $* 15$ & DP74 \\
\hline 6 & DP12 & ${ }^{*} 24$ & *31 & ${ }^{*} 07$ & ${ }^{*} 56$ & ${ }^{\star} 01$ & *14 & \\
\hline 7 & DP13 & ${ }^{*} 31$ & - & $* 40$ & $* 51$ & ${ }^{*} 08$ & ${ }^{*} 09$ & \\
\hline 8 & DP14 & ${ }^{*} 24$ & *31 & $* 15$ & $* 52$ & ${ }^{*} 09$ & $* 15$ & DP74 \\
\hline 9 & DP15 & ${ }^{*} 02$ & ${ }^{*} 02$ & $* 15$ & $* 46$ & ${ }^{*} 08$ & $* 14$ & \\
\hline 10 & DP17 & ${ }^{*} 24$ & ${ }^{*} 26$ & ${ }^{*} 52$ & ${ }^{*} 54$ & ${ }^{*} 09$ & $* 15$ & DP74 \\
\hline 11 & DP20 & ${ }^{*} 26$ & ${ }^{*} 33$ & $* 44$ & $* 46$ & ${ }^{*} 04$ & ${ }^{*} 08$ & \\
\hline 12 & DP25 & ${ }^{*} 02$ & ${ }^{*} 24$ & ${ }^{*} 35$ & ${ }^{*} 51$ & ${ }^{*} 04$ & ${ }^{*} 08$ & \\
\hline 13 & DP26 & ${ }^{*} 02$ & ${ }^{*} 02$ & $* 15$ & $* 35$ & ${ }^{*} 04$ & $* 12$ & \\
\hline 14 & DP28 & ${ }^{\star} 02$ & ${ }^{*} 31$ & $* 35$ & $* 46$ & ${ }^{*} 04$ & ${ }^{*} 08$ & \\
\hline 15 & DP30 & ${ }^{\star} 24$ & *31 & $* 15$ & $* 52$ & ${ }^{*} 09$ & $* 15$ & DP74 \\
\hline 16 & DP31 & *11 & ${ }^{*} 31$ & *48 & $* 55$ & ${ }^{*} 09$ & $* 11$ & \\
\hline 17 & DP32 & ${ }^{*} 24$ & ${ }^{*} 26$ & $* 40$ & $* 40$ & ${ }^{*} 04$ & ${ }^{*} 08$ & \\
\hline 18 & DP33 & *24 & ${ }^{*} 26$ & ${ }^{*} 52$ & $* 54$ & $* 14$ & $* 15$ & DP74 \\
\hline 19 & DP35 & ${ }^{*} 33$ & - & ${ }^{*} 44$ & - & ${ }^{*} 08$ & $* 13$ & \\
\hline 20 & DP38 & $* 11$ & $* 31$ & ${ }^{*} 40$ & *51 & ${ }^{*} 04$ & $* 14$ & \\
\hline 21 & DP39 & *11 & ${ }^{*} 24$ & *15 & $* 40$ & ${ }^{*} 04$ & $* 15$ & DP94 \\
\hline 22 & DP40 & ${ }^{*} 24$ & ${ }^{*} 26$ & *15 & ${ }^{*} 52$ & $* 14$ & $* 15$ & DP74 \\
\hline 23 & DP41 & ${ }^{*} 24$ & ${ }^{*} 26$ & ${ }^{*} 54$ & ${ }^{*} 54$ & ${ }^{*} 01$ & ${ }^{*} 04$ & \\
\hline 24 & DP42 & ${ }^{*} 24$ & ${ }^{*} 24$ & ${ }^{*} 07$ & $* 51$ & ${ }^{*} 01$ & ${ }^{*} 08$ & \\
\hline 25 & DP44 & ${ }^{*} 24$ & ${ }^{*} 24$ & $* 40$ & *52 & $* 12$ & $* 15$ & DP74 \\
\hline 26 & DP46 & *11 & ${ }^{*} 24$ & *15 & ${ }^{*} 51$ & ${ }^{*} 04$ & $* 14$ & DP94 \\
\hline 27 & DP48 & ${ }^{*} 24$ & ${ }^{*} 26$ & *15 & $* 52$ & $* 14$ & $* 15$ & DP74 \\
\hline 28 & DP49 & *24 & ${ }^{*} 33$ & ${ }^{*} 07$ & $* 44$ & ${ }^{*} 01$ & ${ }^{*} 08$ & \\
\hline 29 & DP52 & $* 11$ & ${ }^{*} 26$ & ${ }^{*} 55$ & ${ }^{* 67}$ & ${ }^{*} 08$ & $\star 16$ & \\
\hline 30 & DP53 & ${ }^{*} 24$ & $* 31$ & ${ }^{\star} 07$ & $* 54$ & ${ }^{*} 01$ & ${ }^{*} 04$ & \\
\hline 31 & DP54 & ${ }^{*} 02$ & ${ }^{*} 26$ & $* 40$ & $* 46$ & ${ }^{*} 08$ & ${ }^{*} 09$ & \\
\hline 32 & DP56 & ${ }^{*} 24$ & ${ }^{*} 24$ & $* 40$ & ${ }^{*} 52$ & ${ }^{*} 09$ & $* 15$ & DP74 \\
\hline 33 & DP57 & ${ }^{\star} 24$ & ${ }^{*} 33$ & ${ }^{*} 44$ & ${ }^{*} 54$ & ${ }^{*} 04$ & $* 13$ & \\
\hline 34 & DP58 & *24 & ${ }^{*} 26$ & *40 & $* 40$ & *11 & ${ }^{*} 09$ & \\
\hline 35 & DP59 & ${ }^{*} 24$ & ${ }^{*} 24$ & ${ }^{*} 07$ & $* 15$ & ${ }^{*} 01$ & $* 14$ & \\
\hline 36 & DP60 & ${ }^{*} 02$ & *24 & $* 46$ & $* 52$ & ${ }^{\star} 08$ & *15 & DP74 \\
\hline 37 & DP62 & ${ }^{*} 24$ & ${ }^{*} 24$ & *48 & $* 59$ & ${ }^{*} 04$ & ${ }^{*} 09$ & \\
\hline 38 & DP64 & ${ }^{*} 02$ & ${ }^{*} 02$ & $* 40$ & $* 46$ & ${ }^{*} 08$ & $* 14$ & \\
\hline 39 & DP65 & ${ }^{*} 24$ & ${ }^{*} 24$ & *40 & $* 40$ & ${ }^{*} 09$ & *14 & \\
\hline 40 & DP66 & ${ }^{*} 24$ & ${ }^{*} 24$ & $* 40$ & $* 51$ & ${ }^{*} 08$ & *11 & \\
\hline 41 & DP68 & ${ }^{*} 24$ & *33 & $* 15$ & *55 & ${ }^{*} 04$ & ${ }^{*} 04$ & \\
\hline 42 & DP69 & ${ }^{*} 02$ & *24 & *46 & $* 52$ & ${ }^{*} 09$ & $* 15$ & DP74 \\
\hline 43 & DP72 & ${ }^{*} 02$ & *11 & $* 40$ & $* 46$ & ${ }^{*} 08$ & $* 15$ & \\
\hline 44 & DP73 & ${ }^{*} 02$ & ${ }^{*} 31$ & *15 & $* 40$ & ${ }^{*} 08$ & ${ }^{*} 09$ & \\
\hline 45 & DP74 & ${ }^{*} 24$ & - & ${ }^{*} 52$ & - & $* 15$ & - & DP74 \\
\hline 46 & DP75 & ${ }^{*} 02$ & ${ }^{*} 24$ & $* 15$ & ${ }^{*} 51$ & ${ }^{*} 08$ & - & \\
\hline 47 & DP80 & ${ }^{*} 31$ & ${ }^{*} 33$ & $* 44$ & $* 51$ & ${ }^{*} 09$ & $* 13$ & \\
\hline 48 & DP81 & *24 & ${ }^{*} 24$ & "*07 & $* 52$ & ${ }^{*} 01$ & $* 15$ & DP74 \\
\hline 49 & DP83 & ${ }^{*} 02$ & ${ }^{*} 26$ & $* 15$ & $* 40$ & ${ }^{*} 08$ & $* 15$ & \\
\hline 50 & DP86 & $* 24$ & *31 & ${ }^{*} 51$ & $* 52$ & ${ }^{*} 09$ & $* 15$ & DP74 \\
\hline 51 & DP87 & ${ }^{*} 24$ & ${ }^{*} 33$ & ${ }^{*} 51$ & $* 52$ & $* 14$ & $* 15$ & DP74 \\
\hline 52 & DP92 & ${ }^{*} 02$ & ${ }^{*} 24$ & $* 15$ & $* 35$ & ${ }^{*} 04$ & $* 15$ & \\
\hline 53 & DP94 ${ }^{\#}$ & $* 11$ & - & $* 15$ & - & ${ }^{*} 04$ & - & DP94 \\
\hline 54 & DP95 & ${ }^{*} 24$ & ${ }^{*} 24$ & $\star 35$ & $* 40$ & ${ }^{*} 04$ & ${ }^{*} 09$ & \\
\hline 55 & DP96 & $* 11$ & *24 & $* 54$ & $* 58$ & ${ }^{*} 08$ & $* 13$ & \\
\hline 56 & DP97 & ${ }^{*} 24$ & ${ }^{*} 24$ & ${ }^{*} 52$ & ${ }^{*} 54$ & ${ }^{*} 04$ & $* 15$ & DP74 \\
\hline 57 & DP98 & ${ }^{*} 02$ & *24 & *48 & $* 54$ & ${ }^{*} 04$ & - & \\
\hline 58 & DP99 & ${ }^{*} 24$ & $* 31$ & $* 15$ & $* 52$ & ${ }^{*} 08$ & $* 15$ & DP74 \\
\hline
\end{tabular}




\begin{tabular}{|c|c|c|c|c|c|c|c|c|}
\hline 59 & DP100 & ${ }^{*} 02$ & ${ }^{*} 33$ & ${ }^{*} 35$ & ${ }^{*} 51$ & ${ }^{*} 04$ & ${ }^{*} 04$ & \\
\hline 60 & DP101 & *33 & - & ${ }^{*} 44$ & - & ${ }^{*} 04$ & $* 13$ & \\
\hline 61 & DP105 & ${ }^{*} 02$ & ${ }^{*} 24$ & *35 & ${ }^{*} 40$ & ${ }^{*} 04$ & ${ }^{*} 04$ & \\
\hline 62 & DP106 & ${ }^{*} 24$ & ${ }^{*} 26$ & $* 15$ & $* 40$ & ${ }^{*} 04$ & $* 13$ & \\
\hline 63 & DP109 & ${ }^{*} 24$ & ${ }^{*} 24$ & ${ }^{*} 46$ & ${ }^{*} 54$ & ${ }^{*} 04$ & *15 & \\
\hline 64 & DP111 & $* 33$ & - & ${ }^{*} 44$ & - & ${ }^{*} 09$ & $* 13$ & \\
\hline 65 & DP112 & ${ }^{*} 26$ & ${ }^{*} 33$ & ${ }^{*} 44$ & ${ }^{*} 55$ & $* 13$ & $* 15$ & \\
\hline 66 & DP113 & *11 & ${ }^{*} 24$ & ${ }^{*} 51$ & ${ }^{*} 54$ & ${ }^{*} 04$ & ${ }^{*} 08$ & \\
\hline 67 & DP115 & ${ }^{*} 02$ & ${ }^{*} 24$ & ${ }^{*} 51$ & ${ }^{*} 40$ & ${ }^{*} 09$ & $* 14$ & \\
\hline 68 & DP128 & ${ }^{*} 24$ & ${ }^{*} 24$ & *15 & ${ }^{*} 37$ & ${ }^{*} 09$ & $* 10$ & \\
\hline 69 & DP129 & ${ }^{*} 02$ & $* 11$ & $* 39$ & ${ }^{*} 67$ & ${ }^{*} 04$ & $* 15$ & \\
\hline 70 & DP134 & *31 & ${ }^{*} 33$ & ${ }^{*} 44$ & ${ }^{*} 51$ & ${ }^{*} 04$ & $* 13$ & \\
\hline 71 & DP135 & ${ }^{*} 33$ & - & $* 15$ & ${ }^{*} 44$ & $* 13$ & $* 14$ & \\
\hline 72 & DP136 & *11 & ${ }^{*} 24$ & ${ }^{*} 52$ & ${ }^{*} 54$ & ${ }^{*} 08$ & *15 & DP74 \\
\hline 73 & DP138 & ${ }^{*} 02$ & ${ }^{*} 24$ & ${ }^{*} 40$ & ${ }^{*} 46$ & ${ }^{*} 08$ & $* 12$ & \\
\hline 74 & DP140 & *31 & ${ }^{*} 33$ & *15 & *39 & ${ }^{*} 09$ & $* 15$ & \\
\hline 75 & DP141 & ${ }^{*} 02$ & ${ }^{*} 24$ & ${ }^{*} 40$ & ${ }^{*} 52$ & $* 15$ & $* 15$ & DP74 \\
\hline 76 & DP142 & ${ }^{*} 02$ & ${ }^{*} 33$ & $* 15$ & ${ }^{*} 44$ & ${ }^{*} 09$ & $* 15$ & \\
\hline 77 & DP143 & ${ }^{*} 24$ & ${ }^{*} 24$ & ${ }^{*} 54$ & ${ }^{*} 52$ & $* 14$ & $* 15$ & DP74 \\
\hline 78 & DP144 & ${ }^{*} 02$ & ${ }^{*} 33$ & *44 & - & $* 13$ & - & \\
\hline 79 & DP147 & ${ }^{*} 24$ & ${ }^{*} 24$ & ${ }^{*} 51$ & ${ }^{*} 52$ & ${ }^{*} 04$ & $* 15$ & DP74 \\
\hline 80 & DP153 & ${ }^{*} 02$ & ${ }^{*} 02$ & $* 15$ & $* 55$ & ${ }^{*} 04$ & - & \\
\hline 81 & DP154 & ${ }^{*} 02$ & ${ }^{*} 24$ & *15 & ${ }^{*} 52$ & *15 & $* 15$ & DP74 \\
\hline 82 & DP157 & ${ }^{*} 02$ & ${ }^{*} 33$ & ${ }^{*} 07$ & ${ }^{*} 44$ & ${ }^{*} 01$ & ${ }^{*} 04$ & \\
\hline 83 & DP158 & ${ }^{*} 24$ & ${ }^{*} 33$ & ${ }^{*} 51$ & ${ }^{*} 52$ & ${ }^{*} 09$ & *15 & DP74 \\
\hline 84 & DP159 & ${ }^{*} 02$ & ${ }^{*} 24$ & $* 15$ & ${ }^{*} 46$ & ${ }^{*} 08$ & $* 15$ & \\
\hline 85 & DP160 & ${ }^{*} 02$ & ${ }^{*} 24$ & $* 40$ & ${ }^{*} 54$ & ${ }^{*} 04$ & $* 13$ & \\
\hline 86 & DP163 & ${ }^{*} 24$ & *26 & ${ }^{*} 40$ & ${ }^{*} 46$ & ${ }^{*} 04$ & ${ }^{*} 09$ & \\
\hline 87 & DP164 & ${ }^{*} 02$ & ${ }^{*} 24$ & $* 39$ & $* 40$ & $* 12$ & - & \\
\hline 88 & DP165 & ${ }^{*} 24$ & ${ }^{*} 24$ & ${ }^{*} 07$ & $* 15$ & ${ }^{*} 01$ & ${ }^{*} 09$ & \\
\hline 89 & DP166 & ${ }^{*} 24$ & ${ }^{*} 33$ & ${ }^{*} 40$ & ${ }^{*} 44$ & ${ }^{*} 04$ & $* 16$ & \\
\hline 90 & DP167 & ${ }^{*} 24$ & ${ }^{*} 24$ & ${ }^{*} 40$ & ${ }^{*} 52$ & ${ }^{*} 04$ & $* 15$ & DP74 \\
\hline 91 & DP169 & ${ }^{*} 02$ & ${ }^{*} 26$ & $* 40$ & ${ }^{*} 51$ & ${ }^{*} 08$ & $* 15$ & \\
\hline 92 & DP170 & ${ }^{*} 02$ & ${ }^{*} 24$ & ${ }^{*} 51$ & ${ }^{*} 54$ & ${ }^{*} 04$ & ${ }^{*} 09$ & \\
\hline 93 & DP172 & ${ }^{*} 02$ & ${ }^{*} 24$ & ${ }^{*} 40$ & ${ }^{*} 52$ & ${ }^{*} 04$ & $* 15$ & DP74 \\
\hline 94 & DP173 & ${ }^{*} 01$ & *33 & *37 & ${ }^{*} 44$ & *10 & $* 14$ & \\
\hline 95 & DP174 & ${ }^{*} 02$ & ${ }^{*} 24$ & ${ }^{*} 44$ & ${ }^{*} 48$ & ${ }^{*} 04$ & $* 13$ & \\
\hline 96 & DP175 & ${ }^{*} 02$ & ${ }^{*} 33$ & ${ }^{*} 07$ & ${ }^{*} 44$ & ${ }^{*} 01$ & ${ }^{*} 04$ & \\
\hline 97 & DP176 & $* 24$ & ${ }^{*} 24$ & $* 15$ & $* 40$ & ${ }^{*} 04$ & ${ }^{*} 09$ & \\
\hline 98 & DP177 & $* 24$ & $* 33$ & $* 40$ & $* 44$ & $* 13$ & $* 14$ & \\
\hline 99 & DP178 & ${ }^{*} 24$ & ${ }^{*} 24$ & ${ }^{*} 40$ & ${ }^{*} 52$ & $* 12$ & $* 15$ & DP74 \\
\hline 100 & DP179 & ${ }^{*} 02$ & ${ }^{*} 24$ & *39 & ${ }^{*} 51$ & ${ }^{\star} 08$ & $\star 15$ & \\
\hline 101 & DP181 & ${ }^{*} 02$ & ${ }^{*} 26$ & ${ }^{*} 46$ & ${ }^{*} 48$ & ${ }^{*} 08$ & ${ }^{*} 09$ & \\
\hline 102 & DP182 & *24 & $* 31$ & *27 & *59 & $* 13$ & $* 15$ & \\
\hline 103 & DP184 & ${ }^{*} 24$ & ${ }^{*} 33$ & $* 44$ & ${ }^{*} 52$ & $* 13$ & $* 15$ & DP74 \\
\hline 104 & DP185 & ${ }^{*} 02$ & ${ }^{*} 24$ & *39 & ${ }^{*} 51$ & $* 15$ & - & \\
\hline 105 & DP186 & ${ }^{*} 02$ & ${ }^{* 11}$ & ${ }^{*} 54$ & - & ${ }^{*} 04$ & $* 14$ & \\
\hline 106 & DP187 & ${ }^{*} 02$ & ${ }^{*} 02$ & $* 13$ & $* 46$ & ${ }^{\star} 08$ & $* 12$ & \\
\hline 107 & DP193 & ${ }^{*} 24$ & ${ }^{*} 26$ & $* 39$ & ${ }^{*} 52$ & ${ }^{*} 08$ & $* 15$ & DP74 \\
\hline
\end{tabular}

"DP lines used for induction of pla-iPS cells in this study.

This data was based on the results of 4-digit PCR-rSSOP.

DP74 covers 29, and DP94 covers 3 lines.

Total coverage is $29.9 \%(32 / 107)$. 
Supplementary Table 8. Haplotype frequency for HLA-A, B, and DRB1 loci in Japanese population.

\begin{tabular}{|c|c|c|c|c|c|c|c|}
\hline Rank & HLA-A & HLA-B & HLA-DRB1 & Haplotype Frequency & LD value ${ }^{a}$ & RD value & $\begin{array}{c}\text { Cumulative } \\
\text { matched }\end{array}$ \\
\hline 1 & *24:02 & *52:01 & $\star 15: 02$ & $8.468 \%$ & $8.033 \%$ & 0.786 & $16.922 \%$ \\
\hline 2 & *33:03 & *44:03 & *13:02 & $4.081 \%$ & $4.057 \%$ & 0.737 & $24.285 \%$ \\
\hline 3 & *24:02 & $\star 07: 02$ & *01:01 & $3.746 \%$ & $3.627 \%$ & 0.654 & $31.171 \%$ \\
\hline 4 & *24:02 & $\star 54: 01$ & *04:05 & $2.609 \%$ & $2.238 \%$ & 0.312 & $36.584 \%$ \\
\hline 5 & *02:07 & $\star 46: 01$ & *08:03 & $2.003 \%$ & $1.988 \%$ & 0.573 & $39.908 \%$ \\
\hline 6 & *11:01 & *15:01 & ${ }^{\star} 04: 06$ & $1.281 \%$ & $1.259 \%$ & 0.397 & $42.065 \%$ \\
\hline 7 & *24:02 & *59:01 & ${ }^{*} 04: 05$ & $1.062 \%$ & $0.968 \%$ & 0.535 & $43.797 \%$ \\
\hline 8 & *11:01 & *54:01 & ${ }^{*} 04: 05$ & $0.958 \%$ & $0.866 \%$ & 0.116 & $45.112 \%$ \\
\hline 9 & *24:02 & *40:06 & ${ }^{\star} 09: 01$ & $0.866 \%$ & $0.630 \%$ & 0.146 & $47.274 \%$ \\
\hline 10 & *26:01 & *40:02 & $\star 09: 01$ & $0.843 \%$ & $0.754 \%$ & 0.100 & $49.000 \%$ \\
\hline 11 & *24:02 & *51:01 & ${ }^{\star} 09: 01$ & $0.756 \%$ & $0.274 \%$ & 0.031 & $50.972 \%$ \\
\hline 12 & *31:01 & *51:01 & ${ }^{\star} 08: 02$ & $0.606 \%$ & $0.571 \%$ & 0.134 & $52.049 \%$ \\
\hline 13 & *26:02 & *40:06 & ${ }^{\star} 09: 01$ & $0.600 \%$ & $0.588 \%$ & 0.319 & $52.738 \%$ \\
\hline 14 & *24:02 & *46:01 & ${ }^{\star} 08: 03$ & $0.548 \%$ & $0.397 \%$ & 0.084 & $53.795 \%$ \\
\hline 15 & *02:01 & *13:01 & *12:02 & $0.468 \%$ & $0.465 \%$ & 0.383 & $54.578 \%$ \\
\hline 15 & *11:01 & *39:01 & *08:03 & $0.468 \%$ & $0.441 \%$ & 0.129 & $55.333 \%$ \\
\hline 17 & *24:02 & *40:01 & ${ }^{*} 09: 01$ & $0.456 \%$ & $0.179 \%$ & 0.035 & $56.329 \%$ \\
\hline 17 & *26:01 & *40:02 & ${ }^{*} 08: 02$ & $0.456 \%$ & $0.430 \%$ & 0.101 & $56.996 \%$ \\
\hline 19 & *33:03 & *44:03 & *08:03 & $0.450 \%$ & $0.413 \%$ & 0.067 & $57.691 \%$ \\
\hline 20 & ${ }^{\star} 02: 06$ & $\star 35: 01$ & *15:01 & $0.427 \%$ & $0.369 \%$ & 0.048 & $58.672 \%$ \\
\hline 21 & *02:06 & *40:06 & *09:01 & $0.416 \%$ & $0.355 \%$ & 0.079 & $59.265 \%$ \\
\hline 22 & *02:06 & *39:01 & *15:01 & $0.398 \%$ & $0.374 \%$ & 0.110 & $59.884 \%$ \\
\hline 22 & *24:02 & *52:01 & ${ }^{\star} 09: 01$ & $0.398 \%$ & $-0.187 \%$ & -0.017 & $60.413 \%$ \\
\hline 24 & *24:02 & $\star 40: 02$ & ${ }^{\star} 09: 01$ & $0.392 \%$ & $-0.024 \%$ & -0.003 & $61.198 \%$ \\
\hline 25 & *31:01 & *51:01 & *14:03 & $0.381 \%$ & $0.368 \%$ & 0.232 & $61.788 \%$ \\
\hline 26 & *02:07 & *46:01 & ${ }^{\star 09: 01}$ & $0.375 \%$ & $0.351 \%$ & 0.101 & $62.258 \%$ \\
\hline 27 & ${ }^{\star} 02: 01$ & *54:01 & ${ }^{\star 04: 05}$ & $0.364 \%$ & $0.244 \%$ & 0.033 & $62.802 \%$ \\
\hline 28 & *11:01 & *67:01 & $\star 16: 02$ & $0.352 \%$ & $0.351 \%$ & 0.360 & $63.275 \%$ \\
\hline 29 & $\star 24: 02$ & *40:01 & *04:05 & $0.346 \%$ & $0.084 \%$ & 0.016 & $63.999 \%$ \\
\hline 29 & *26:03 & *15:01 & *15:01 & $0.346 \%$ & $0.333 \%$ & 0.148 & $64.554 \%$ \\
\hline 31 & *26:01 & *35:01 & ${ }^{*} 04: 10$ & $0.335 \%$ & $0.322 \%$ & 0.154 & $65.063 \%$ \\
\hline 32 & *33:03 & *58:01 & *13:02 & $0.329 \%$ & $0.327 \%$ & 0.536 & $65.480 \%$ \\
\hline 33 & *31:01 & *51:01 & *09:01 & $0.323 \%$ & $0.205 \%$ & 0.023 & $66.096 \%$ \\
\hline 34 & $\star 24: 02$ & $\star 15: 18$ & ${ }^{\star} 04: 01$ & $0.312 \%$ & $0.307 \%$ & 0.345 & $66.524 \%$ \\
\hline 34 & *26:03 & *15:01 & ${ }^{\star} 09: 01$ & $0.312 \%$ & $0.286 \%$ & 0.128 & $66.918 \%$ \\
\hline 36 & *26:02 & *15:01 & $\star 14: 06$ & $0.306 \%$ & $0.304 \%$ & 0.239 & $67.307 \%$ \\
\hline 37 & $\star 02: 01$ & $\star 15: 11$ & ${ }^{\star} 09: 01$ & $0.300 \%$ & $0.284 \%$ & 0.294 & $67.688 \%$ \\
\hline 37 & $\star 24: 02$ & $\star 35: 01$ & ${ }^{\star} 09: 01$ & $0.300 \%$ & $-0.120 \%$ & -0.016 & $68.269 \%$ \\
\hline 37 & *24:02 & *51:01 & *14:03 & $0.300 \%$ & $0.247 \%$ & 0.160 & $68.676 \%$ \\
\hline 40 & $\star 02: 01$ & $* 07: 02$ & *01:01 & $0.294 \%$ & $0.256 \%$ & 0.045 & $69.112 \%$ \\
\hline 40 & *02:01 & *40:06 & *09:01 & $0.294 \%$ & $0.219 \%$ & 0.049 & $69.429 \%$ \\
\hline 40 & $\star 24: 02$ & *15:01 & $\star 04: 06$ & $0.294 \%$ & $0.205 \%$ & 0.066 & $69.913 \%$ \\
\hline 40 & $\star 24: 02$ & *40:02 & *15:01 & $0.294 \%$ & $0.069 \%$ & 0.009 & $70.568 \%$ \\
\hline 40 & *31:01 & *51:01 & ${ }^{\star} 04: 05$ & $0.294 \%$ & $0.182 \%$ & 0.021 & $71.099 \%$ \\
\hline 45 & $\star 02: 06$ & *59:01 & $\star 04: 05$ & $0.283 \%$ & $0.259 \%$ & 0.138 & $71.382 \%$ \\
\hline 46 & $\star 02: 06$ & *54:01 & ${ }^{\star} 04: 05$ & $0.277 \%$ & $0.182 \%$ & 0.024 & $71.629 \%$ \\
\hline 47 & *01:01 & *37:01 & *10:01 & $0.266 \%$ & $0.266 \%$ & 0.568 & $71.949 \%$ \\
\hline 47 & *24:02 & *44:03 & *13:02 & $0.266 \%$ & $0.141 \%$ & 0.026 & $72.311 \%$ \\
\hline 49 & *24:02 & *15:07 & ${ }^{\star} 04: 03$ & $0.260 \%$ & $0.254 \%$ & 0.435 & $72.609 \%$ \\
\hline 49 & *24:02 & *35:01 & *15:01 & $0.260 \%$ & $0.033 \%$ & 0.004 & $73.118 \%$ \\
\hline 51 & $\star 02: 01$ & *40:02 & ${ }^{\star} 09: 01$ & $0.248 \%$ & $0.114 \%$ & 0.014 & $73.488 \%$ \\
\hline
\end{tabular}




\begin{tabular}{|c|c|c|c|c|c|c|c|}
\hline 51 & ${ }^{\star} 02: 01$ & $\star 51: 01$ & *15:01 & $0.248 \%$ & $0.164 \%$ & 0.021 & $73.968 \%$ \\
\hline 51 & *11:01 & $\star 54: 01$ & $\star^{\star} 08: 03$ & $0.248 \%$ & $0.190 \%$ & 0.025 & $74.274 \%$ \\
\hline 51 & $\star 24: 02$ & $\star 40: 02$ & ${ }^{*} 04: 05$ & $0.248 \%$ & $-0.147 \%$ & -0.019 & $74.732 \%$ \\
\hline 51 & *24:02 & *51:01 & ${ }^{*} 04: 05$ & $0.248 \%$ & $-0.209 \%$ & -0.024 & $75.115 \%$ \\
\hline 56 & ${ }^{\star} 02: 06$ & *35:01 & ${ }^{*} 09: 01$ & $0.242 \%$ & $0.134 \%$ & 0.017 & $75.470 \%$ \\
\hline 56 & *11:01 & *35:01 & ${ }^{*} 04: 05$ & $0.242 \%$ & $0.143 \%$ & 0.018 & $75.847 \%$ \\
\hline 56 & *24:02 & *51:01 & *15:01 & $0.242 \%$ & $-0.018 \%$ & -0.002 & $76.149 \%$ \\
\hline 59 & *11:01 & *55:02 & ${ }^{*} 04: 05$ & $0.237 \%$ & $0.207 \%$ & 0.085 & $76.418 \%$ \\
\hline 59 & *31:01 & *52:01 & *15:02 & $0.237 \%$ & $0.130 \%$ & 0.015 & $76.745 \%$ \\
\hline 61 & *03:01 & *44:02 & *13:01 & $0.231 \%$ & $0.231 \%$ & 0.606 & $77.007 \%$ \\
\hline 62 & ${ }^{*} 02: 01$ & *54:01 & ${ }^{\star} 08: 03$ & $0.225 \%$ & $0.149 \%$ & 0.020 & $77.270 \%$ \\
\hline 62 & *02:06 & *51:01 & ${ }^{\star} 09: 01$ & $0.225 \%$ & $0.101 \%$ & 0.011 & $77.507 \%$ \\
\hline 62 & ${ }^{*} 02: 10$ & *40:06 & ${ }^{*} 04: 05$ & $0.225 \%$ & $0.223 \%$ & 0.547 & $77.725 \%$ \\
\hline 62 & *24:02 & *15:01 & *09:01 & $0.225 \%$ & $-0.177 \%$ & -0.024 & $78.009 \%$ \\
\hline 62 & *24:02 & *35:01 & ${ }^{*} 04: 05$ & $0.225 \%$ & $-0.174 \%$ & -0.023 & $78.288 \%$ \\
\hline 62 & *26:01 & *54:01 & ${ }^{*} 04: 05$ & $0.225 \%$ & $0.146 \%$ & 0.020 & $78.421 \%$ \\
\hline 68 & ${ }^{\star} 02: 01$ & $\star 46: 01$ & ${ }^{\star} 08: 03$ & $0.219 \%$ & $0.171 \%$ & 0.035 & $78.698 \%$ \\
\hline 68 & ${ }^{\star} 02: 06$ & $\star 39: 01$ & ${ }^{\star} 08: 02$ & $0.219 \%$ & $0.206 \%$ & 0.060 & $78.941 \%$ \\
\hline 68 & *24:02 & $\star 40: 02$ & *14:54 & $0.219 \%$ & $0.119 \%$ & 0.035 & $79.271 \%$ \\
\hline 68 & *24:02 & *48:01 & *15:01 & $0.219 \%$ & $0.140 \%$ & 0.051 & $79.514 \%$ \\
\hline 68 & *26:01 & $\star 40: 06$ & ${ }^{\star} 09: 01$ & $0.219 \%$ & $0.169 \%$ & 0.038 & $79.639 \%$ \\
\hline 73 & ${ }^{\star} 02: 06$ & *35:01 & ${ }^{\star} 04: 03$ & $0.214 \%$ & $0.193 \%$ & 0.070 & $79.899 \%$ \\
\hline 73 & ${ }^{\star} 02: 06$ & *48:01 & ${ }^{\star} 04: 07$ & $0.214 \%$ & $0.212 \%$ & 0.429 & $80.116 \%$ \\
\hline 73 & *24:02 & *13:01 & ${ }^{\star} 12: 02$ & $0.214 \%$ & $0.206 \%$ & 0.170 & $80.345 \%$ \\
\hline 73 & *31:01 & *56:01 & ${ }^{*} 09: 01$ & $0.214 \%$ & $0.202 \%$ & 0.223 & $80.538 \%$ \\
\hline 77 & ${ }^{*} 02: 01$ & *35:01 & ${ }^{*} 04: 10$ & $0.208 \%$ & $0.188 \%$ & 0.090 & $80.768 \%$ \\
\hline 77 & *24:02 & *40:02 & ${ }^{*} 08: 02$ & $0.208 \%$ & $0.084 \%$ & 0.020 & $81.001 \%$ \\
\hline 77 & *26:01 & ${ }^{\star} 07: 02$ & ${ }^{\star} 01: 01$ & $0.208 \%$ & $0.183 \%$ & 0.032 & $81.192 \%$ \\
\hline 80 & ${ }^{\star} 02: 06$ & ${ }^{\star} 07: 02$ & ${ }^{*} 01: 01$ & $0.202 \%$ & $0.171 \%$ & 0.030 & $81.344 \%$ \\
\hline 80 & *11:01 & *67:01 & *15:01 & $0.202 \%$ & $0.194 \%$ & 0.170 & $81.539 \%$ \\
\hline 80 & *26:01 & *52:01 & *15:02 & $0.202 \%$ & $0.110 \%$ & 0.015 & $81.744 \%$ \\
\hline 83 & ${ }^{*} 02: 01$ & *40:01 & ${ }^{*} 04: 05$ & $0.196 \%$ & $0.112 \%$ & 0.021 & $82.016 \%$ \\
\hline 83 & ${ }^{*} 02: 01$ & *40:01 & ${ }^{*} 09: 01$ & $0.196 \%$ & $0.107 \%$ & 0.020 & $82.229 \%$ \\
\hline 83 & ${ }^{*} 02: 01$ & $\star 51: 01$ & ${ }^{*} 09: 01$ & $0.196 \%$ & $0.041 \%$ & 0.004 & $82.385 \%$ \\
\hline 83 & *02:06 & *15:01 & *15:01 & $0.196 \%$ & $0.140 \%$ & 0.018 & $82.633 \%$ \\
\hline 83 & *24:02 & *35:01 & ${ }^{*} 04: 03$ & $0.196 \%$ & $0.115 \%$ & 0.043 & $82.865 \%$ \\
\hline 83 & *24:02 & *40:01 & *11:01 & $0.196 \%$ & $0.142 \%$ & 0.052 & $83.099 \%$ \\
\hline 83 & *31:01 & *40:02 & ${ }^{*} 04: 10$ & $0.196 \%$ & $0.181 \%$ & 0.087 & $83.307 \%$ \\
\hline 83 & *31:01 & $\star 40: 02$ & *14:54 & $0.196 \%$ & $0.171 \%$ & 0.050 & $83.515 \%$ \\
\hline 83 & *31:01 & $\star 54: 01$ & ${ }^{\star} 04: 05$ & $0.196 \%$ & $0.105 \%$ & 0.014 & $83.583 \%$ \\
\hline 92 & ${ }^{\star} 02: 06$ & *52:01 & *15:02 & $0.190 \%$ & $0.079 \%$ & 0.009 & $83.742 \%$ \\
\hline 92 & *24:02 & *40:01 & *15:01 & $0.190 \%$ & $0.041 \%$ & 0.008 & $83.913 \%$ \\
\hline 92 & *24:02 & *52:01 & ${ }^{*} 04: 05$ & $0.190 \%$ & $-0.365 \%$ & -0.034 & $84.033 \%$ \\
\hline 92 & *31:01 & *51:01 & *14:54 & $0.190 \%$ & $0.162 \%$ & 0.047 & $84.207 \%$ \\
\hline 96 & *24:02 & *51:01 & *11:01 & $0.185 \%$ & $0.091 \%$ & 0.034 & $84.397 \%$ \\
\hline 96 & *24:02 & *54:01 & ${ }^{*} 08: 03$ & $0.185 \%$ & $-0.050 \%$ & -0.007 & $84.552 \%$ \\
\hline 96 & *31:01 & *40:02 & ${ }^{*} 08: 02$ & $0.185 \%$ & $0.154 \%$ & 0.036 & $84.687 \%$ \\
\hline 99 & ${ }^{*} 02: 01$ & *35:01 & ${ }^{*} 09: 01$ & $0.179 \%$ & $0.044 \%$ & 0.005 & $84.873 \%$ \\
\hline 99 & *24:02 & *40:06 & *12:01 & $0.179 \%$ & $0.119 \%$ & 0.033 & $85.060 \%$ \\
\hline 99 & *24:02 & *51:01 & *12:01 & $0.179 \%$ & $0.057 \%$ & 0.016 & $85.262 \%$ \\
\hline 99 & *26:01 & ${ }^{\star} 40: 02$ & *14:54 & $0.179 \%$ & $0.158 \%$ & 0.046 & $85.391 \%$ \\
\hline 103 & ${ }^{\star} 02: 01$ & $\star 51: 01$ & *14:03 & $0.173 \%$ & $0.156 \%$ & 0.099 & $85.506 \%$ \\
\hline 103 & ${ }^{\star} 02: 06$ & *46:01 & ${ }^{\star} 08: 03$ & $0.173 \%$ & $0.134 \%$ & 0.028 & $85.641 \%$ \\
\hline 103 & *11:01 & $\star 51: 01$ & *04:05 & $0.173 \%$ & $0.059 \%$ & 0.007 & $85.771 \%$ \\
\hline
\end{tabular}




\begin{tabular}{|c|c|c|c|c|c|c|c|}
\hline 103 & $\star 24: 02$ & *35:01 & ${ }^{\star} 08: 02$ & $0.173 \%$ & $0.048 \%$ & 0.011 & $85.949 \%$ \\
\hline 103 & $\star 24: 02$ & *55:02 & *04:05 & $0.173 \%$ & $0.052 \%$ & 0.022 & $86.076 \%$ \\
\hline 103 & $\star 26: 01$ & *15:01 & $\star^{\star} 04: 06$ & $0.173 \%$ & $0.154 \%$ & 0.049 & $86.219 \%$ \\
\hline 103 & *26:01 & *35:01 & *15:01 & $0.173 \%$ & $0.125 \%$ & 0.016 & $86.400 \%$ \\
\hline 103 & *31:01 & *15:01 & *15:01 & $0.173 \%$ & $0.120 \%$ & 0.016 & $86.586 \%$ \\
\hline 111 & *02:01 & *52:01 & *15:02 & $0.167 \%$ & $0.027 \%$ & 0.003 & $86.711 \%$ \\
\hline 111 & *11:01 & *15:01 & *09:01 & $0.167 \%$ & $0.067 \%$ & 0.009 & $86.883 \%$ \\
\hline 111 & *24:02 & *40:02 & *14:05 & $0.167 \%$ & $0.110 \%$ & 0.057 & $87.010 \%$ \\
\hline 111 & *26:01 & *35:01 & ${ }^{\star} 08: 02$ & $0.167 \%$ & $0.141 \%$ & 0.033 & $87.129 \%$ \\
\hline 111 & *30:01 & *13:02 & ${ }^{\star} 07: 01$ & $0.167 \%$ & $0.167 \%$ & 0.806 & $87.283 \%$ \\
\hline 111 & *31:01 & *07:02 & ${ }^{\star} 01: 01$ & $0.167 \%$ & $0.138 \%$ & 0.024 & $87.369 \%$ \\
\hline 117 & *02:01 & *15:01 & ${ }^{\star} 09: 01$ & $0.162 \%$ & $0.032 \%$ & 0.004 & $87.479 \%$ \\
\hline 117 & *24:02 & *35:01 & *12:01 & $0.162 \%$ & $0.055 \%$ & 0.016 & $87.626 \%$ \\
\hline 117 & *24:02 & *40:01 & *12:01 & $0.162 \%$ & $0.092 \%$ & 0.026 & $87.748 \%$ \\
\hline 117 & *24:02 & *54:01 & *01:01 & $0.162 \%$ & $0.003 \%$ & 0.001 & $87.850 \%$ \\
\hline 117 & *31:01 & *35:01 & *09:01 & $0.162 \%$ & $0.058 \%$ & 0.007 & $87.961 \%$ \\
\hline 117 & *31:01 & *40:01 & ${ }^{*} 08: 03$ & $0.162 \%$ & $0.121 \%$ & 0.023 & $88.145 \%$ \\
\hline 117 & $\star 33: 03$ & $\star 44: 03$ & ${ }^{\star} 09: 01$ & $0.162 \%$ & $0.099 \%$ & 0.016 & $88.308 \%$ \\
\hline 124 & $\star 02: 01$ & *48:01 & *14:54 & $0.156 \%$ & $0.145 \%$ & 0.052 & $88.456 \%$ \\
\hline 124 & $\star 02: 06$ & *40:02 & ${ }^{*} 09: 01$ & $0.156 \%$ & $0.049 \%$ & 0.006 & $88.547 \%$ \\
\hline 124 & *11:01 & *46:01 & ${ }^{\star} 08: 03$ & $0.156 \%$ & $0.118 \%$ & 0.024 & $88.625 \%$ \\
\hline 124 & $\star 24: 02$ & $\star 40: 01$ & *14:54 & $0.156 \%$ & $0.089 \%$ & 0.026 & $88.731 \%$ \\
\hline 124 & *31:01 & *40:02 & ${ }^{\star} 04: 05$ & $0.156 \%$ & $0.059 \%$ & 0.007 & $88.856 \%$ \\
\hline 129 & ${ }^{\star} 02: 01$ & $\star 35: 01$ & ${ }^{\star} 12: 01$ & $0.150 \%$ & $0.116 \%$ & 0.032 & $89.008 \%$ \\
\hline 129 & $\star 02: 01$ & *35:01 & *15:01 & $0.150 \%$ & $0.077 \%$ & 0.010 & $89.114 \%$ \\
\hline 129 & *24:02 & *39:01 & ${ }^{*} 08: 03$ & $0.150 \%$ & $0.043 \%$ & 0.013 & $89.275 \%$ \\
\hline 129 & *24:02 & *40:02 & *11:01 & $0.150 \%$ & $0.069 \%$ & 0.025 & $89.370 \%$ \\
\hline 129 & *24:02 & *46:01 & ${ }^{*} 09: 01$ & $0.150 \%$ & $-0.102 \%$ & -0.022 & $89.441 \%$ \\
\hline 129 & *24:02 & *51:01 & ${ }^{\star} 08: 02$ & $0.150 \%$ & $0.006 \%$ & 0.002 & $89.527 \%$ \\
\hline 129 & *31:01 & *40:02 & *11:01 & $0.150 \%$ & $0.130 \%$ & 0.047 & $89.621 \%$ \\
\hline 136 & *02:06 & *39:01 & ${ }^{*} 08: 03$ & $0.144 \%$ & $0.117 \%$ & 0.034 & $89.716 \%$ \\
\hline 136 & *02:06 & *51:01 & *12:01 & $0.144 \%$ & $0.113 \%$ & 0.031 & $89.833 \%$ \\
\hline 136 & *11:01 & *40:01 & ${ }^{*} 08: 03$ & $0.144 \%$ & $0.103 \%$ & 0.019 & $89.940 \%$ \\
\hline 136 & $\star 31: 01$ & *40:02 & ${ }^{*} 09: 01$ & $0.144 \%$ & $0.042 \%$ & 0.005 & $89.979 \%$ \\
\hline 136 & $\star 31: 01$ & *51:01 & *14:05 & $0.144 \%$ & $0.128 \%$ & 0.065 & $90.074 \%$ \\
\hline 141 & ${ }^{\star} 02: 01$ & *35:01 & ${ }^{*} 08: 02$ & $0.139 \%$ & $0.098 \%$ & 0.023 & $90.154 \%$ \\
\hline 141 & ${ }^{\star} 02: 01$ & $\star 55: 02$ & ${ }^{\star} 04: 05$ & $0.139 \%$ & $0.100 \%$ & 0.041 & $90.251 \%$ \\
\hline 141 & $\star 02: 06$ & *40:01 & ${ }^{\star} 04: 05$ & $0.139 \%$ & $0.071 \%$ & 0.013 & $90.346 \%$ \\
\hline 141 & *11:01 & *48:01 & ${ }^{\star} 09: 01$ & $0.139 \%$ & $0.102 \%$ & 0.037 & $90.455 \%$ \\
\hline 141 & *11:01 & $\star 52: 01$ & *15:02 & $0.139 \%$ & $0.030 \%$ & 0.003 & $90.508 \%$ \\
\hline 141 & $\star 24: 02$ & *40:06 & ${ }^{\star} 04: 05$ & $0.139 \%$ & $-0.085 \%$ & -0.020 & $90.575 \%$ \\
\hline 141 & *24:02 & *54:01 & *13:01 & $0.139 \%$ & $0.123 \%$ & 0.219 & $90.648 \%$ \\
\hline 141 & *31:01 & *51:01 & *11:01 & $0.139 \%$ & $0.115 \%$ & 0.042 & $90.714 \%$ \\
\hline 149 & ${ }^{\star} 02: 01$ & *54:01 & *15:01 & $0.133 \%$ & $0.065 \%$ & 0.009 & $90.802 \%$ \\
\hline 149 & ${ }^{\star} 02: 06$ & *35:01 & ${ }^{*} 04: 05$ & $0.133 \%$ & $0.030 \%$ & 0.004 & $90.885 \%$ \\
\hline 149 & *24:02 & *15:01 & *15:01 & $0.133 \%$ & $-0.085 \%$ & -0.011 & $90.983 \%$ \\
\hline 149 & *24:02 & *52:01 & *15:01 & $0.133 \%$ & $-0.184 \%$ & -0.025 & $91.034 \%$ \\
\hline 149 & *26:01 & *40:02 & ${ }^{*} 04: 05$ & $0.133 \%$ & $0.049 \%$ & 0.006 & $91.120 \%$ \\
\hline 149 & *31:01 & *35:01 & ${ }^{*} 04: 03$ & $0.133 \%$ & $0.113 \%$ & 0.041 & $91.201 \%$ \\
\hline 149 & *31:01 & *51:01 & *15:01 & $0.133 \%$ & $0.069 \%$ & 0.009 & $91.259 \%$ \\
\hline 156 & *02:06 & *40:02 & ${ }^{\star} 08: 02$ & $0.127 \%$ & $0.095 \%$ & 0.022 & $91.326 \%$ \\
\hline 156 & *02:06 & *40:02 & *15:01 & $0.127 \%$ & $0.069 \%$ & 0.009 & $91.399 \%$ \\
\hline 156 & *11:01 & *56:03 & $\star 12: 01$ & $0.127 \%$ & $0.126 \%$ & 0.563 & $91.488 \%$ \\
\hline 156 & *24:02 & *35:01 & *14:54 & $0.127 \%$ & $0.025 \%$ & 0.008 & $91.568 \%$ \\
\hline
\end{tabular}




\begin{tabular}{|c|c|c|c|c|c|c|c|}
\hline 156 & *24:02 & *48:01 & ${ }^{*} 04: 05$ & $0.127 \%$ & $-0.012 \%$ & -0.004 & $91.635 \%$ \\
\hline 156 & *24:02 & *52:01 & ${ }^{\star} 08: 03$ & $0.127 \%$ & $-0.225 \%$ & -0.027 & $91.701 \%$ \\
\hline 156 & *24:02 & *54:01 & ${ }^{\star} 09: 01$ & $0.127 \%$ & $-0.264 \%$ & -0.037 & $91.772 \%$ \\
\hline 156 & *26:01 & $\star 35: 01$ & ${ }^{\star} 04: 03$ & $0.127 \%$ & $0.110 \%$ & 0.040 & $91.836 \%$ \\
\hline 156 & *33:03 & *44:03 & ${ }^{\star} 04: 05$ & $0.127 \%$ & $0.068 \%$ & 0.011 & $91.940 \%$ \\
\hline 165 & ${ }^{\star} 02: 01$ & *39:01 & *15:01 & $0.121 \%$ & $0.090 \%$ & 0.027 & $92.028 \%$ \\
\hline 165 & ${ }^{*} 02: 06$ & $\star 15: 01$ & ${ }^{*} 09: 01$ & $0.121 \%$ & $0.018 \%$ & 0.002 & $92.080 \%$ \\
\hline 165 & *24:02 & *07:02 & ${ }^{*} 09: 01$ & $0.121 \%$ & $-0.173 \%$ & -0.032 & $92.131 \%$ \\
\hline 165 & *24:02 & *40:02 & ${ }^{*} 04: 10$ & $0.121 \%$ & $0.060 \%$ & 0.029 & $92.190 \%$ \\
\hline 165 & *26:01 & *35:01 & *14:54 & $0.121 \%$ & $0.100 \%$ & 0.029 & $92.251 \%$ \\
\hline 165 & *31:01 & *40:06 & ${ }^{*} 09: 01$ & $0.121 \%$ & $0.063 \%$ & 0.014 & $92.283 \%$ \\
\hline 171 & ${ }^{*} 02: 01$ & *40:02 & *15:01 & $0.115 \%$ & $0.043 \%$ & 0.006 & $92.351 \%$ \\
\hline 171 & *02:01 & *51:01 & *14:54 & $0.115 \%$ & $0.078 \%$ & 0.023 & $92.434 \%$ \\
\hline 171 & *02:06 & *35:01 & *11:01 & $0.115 \%$ & $0.094 \%$ & 0.034 & $92.501 \%$ \\
\hline 171 & *11:01 & *07:02 & ${ }^{*} 01: 01$ & $0.115 \%$ & $0.086 \%$ & 0.015 & $92.537 \%$ \\
\hline 171 & *24:02 & *07:02 & ${ }^{*} 04: 05$ & $0.115 \%$ & $-0.163 \%$ & -0.030 & $92.574 \%$ \\
\hline 171 & *24:02 & *15:01 & *14:06 & $0.115 \%$ & $0.080 \%$ & 0.064 & $92.664 \%$ \\
\hline 171 & $\star 24: 02$ & *40:01 & ${ }^{\star} 08: 03$ & $0.115 \%$ & $-0.051 \%$ & -0.010 & $92.724 \%$ \\
\hline 171 & $\star 24: 02$ & *40:01 & *14:05 & $0.115 \%$ & $0.077 \%$ & 0.040 & $92.773 \%$ \\
\hline 171 & *26:01 & *40:01 & ${ }^{*} 12: 01$ & $0.115 \%$ & $0.101 \%$ & 0.028 & $92.853 \%$ \\
\hline 171 & *26:03 & *35:01 & ${ }^{\star} 04: 03$ & $0.115 \%$ & $0.110 \%$ & 0.049 & $92.892 \%$ \\
\hline 181 & ${ }^{*} 02: 01$ & *35:01 & ${ }^{\star} 04: 03$ & $0.110 \%$ & $0.083 \%$ & 0.030 & $92.920 \%$ \\
\hline 181 & ${ }^{\star} 02: 01$ & *38:02 & ${ }^{\star} 08: 03$ & $0.110 \%$ & $0.107 \%$ & 0.390 & $92.983 \%$ \\
\hline 181 & ${ }^{\star} 02: 01$ & *40:01 & ${ }^{\star} 08: 03$ & $0.110 \%$ & $0.056 \%$ & 0.011 & $93.039 \%$ \\
\hline 181 & ${ }^{*} 02: 06$ & *51:01 & ${ }^{*} 04: 05$ & $0.110 \%$ & $-0.008 \%$ & -0.001 & $93.088 \%$ \\
\hline 181 & *11:01 & *15:01 & ${ }^{*} 04: 05$ & $0.110 \%$ & $0.015 \%$ & 0.002 & $93.161 \%$ \\
\hline 181 & *11:01 & *15:01 & *15:01 & $0.110 \%$ & $0.056 \%$ & 0.007 & $93.210 \%$ \\
\hline 181 & *11:01 & *39:01 & ${ }^{*} 04: 05$ & $0.110 \%$ & $0.068 \%$ & 0.020 & $93.255 \%$ \\
\hline 181 & *33:03 & *44:03 & *15:01 & $0.110 \%$ & $0.076 \%$ & 0.012 & $93.327 \%$ \\
\hline 189 & ${ }^{*} 02: 01$ & *39:01 & ${ }^{*} 08: 03$ & $0.104 \%$ & $0.070 \%$ & 0.020 & $93.376 \%$ \\
\hline 189 & ${ }^{*} 02: 01$ & *40:01 & *15:01 & $0.104 \%$ & $0.056 \%$ & 0.010 & $93.419 \%$ \\
\hline 189 & *24:02 & *40:02 & ${ }^{*} 08: 03$ & $0.104 \%$ & $-0.146 \%$ & -0.019 & $93.462 \%$ \\
\hline 189 & *24:02 & *40:02 & *12:01 & $0.104 \%$ & $-0.002 \%$ & 0.000 & $93.496 \%$ \\
\hline 189 & *24:02 & *51:01 & *14:54 & $0.104 \%$ & $-0.013 \%$ & -0.004 & $93.539 \%$ \\
\hline 189 & *26:01 & *39:01 & ${ }^{*} 08: 03$ & $0.104 \%$ & $0.081 \%$ & 0.024 & $93.576 \%$ \\
\hline 189 & *26:01 & *40:01 & *15:01 & $0.104 \%$ & $0.072 \%$ & 0.014 & $93.626 \%$ \\
\hline 189 & *31:01 & *13:01 & ${ }^{\star} 12: 02$ & $0.104 \%$ & $0.102 \%$ & 0.084 & $93.682 \%$ \\
\hline 189 & *31:01 & *15:01 & ${ }^{\star} 04: 06$ & $0.104 \%$ & $0.082 \%$ & 0.026 & $93.735 \%$ \\
\hline 198 & ${ }^{\star} 02: 01$ & *15:01 & *11:01 & $0.098 \%$ & $0.073 \%$ & 0.026 & $93.809 \%$ \\
\hline 198 & ${ }^{\star} 02: 01$ & *15:01 & ${ }^{\star} 15: 01$ & $0.098 \%$ & $0.028 \%$ & 0.004 & $93.838 \%$ \\
\hline 198 & ${ }^{\star} 02: 01$ & *15:18 & ${ }^{\star} 04: 01$ & $0.098 \%$ & $0.097 \%$ & 0.108 & $93.896 \%$ \\
\hline 198 & ${ }^{*} 02: 01$ & *35:01 & *14:54 & $0.098 \%$ & $0.065 \%$ & 0.019 & $93.928 \%$ \\
\hline 198 & ${ }^{\star} 02: 01$ & *40:02 & ${ }^{\star} 08: 02$ & $0.098 \%$ & $0.058 \%$ & 0.014 & $93.963 \%$ \\
\hline 198 & ${ }^{*} 02: 06$ & *48:01 & ${ }^{\star} 09: 01$ & $0.098 \%$ & $0.061 \%$ & 0.022 & $94.018 \%$ \\
\hline 198 & *24:02 & *35:01 & ${ }^{\star} 04: 10$ & $0.098 \%$ & $0.037 \%$ & 0.018 & $94.055 \%$ \\
\hline 198 & *24:02 & *48:01 & ${ }^{\star} 09: 01$ & $0.098 \%$ & $-0.048 \%$ & -0.018 & $94.093 \%$ \\
\hline 198 & *24:02 & *55:02 & ${ }^{\star} 15: 01$ & $0.098 \%$ & $0.029 \%$ & 0.012 & $94.138 \%$ \\
\hline 198 & *24:02 & *67:01 & *16:02 & $0.098 \%$ & $0.094 \%$ & 0.097 & $94.191 \%$ \\
\hline 198 & *26:01 & *35:01 & ${ }^{*} 09: 01$ & $0.098 \%$ & $0.009 \%$ & 0.001 & $94.219 \%$ \\
\hline 198 & *26:01 & *46:01 & ${ }^{*} 08: 03$ & $0.098 \%$ & $0.066 \%$ & 0.014 & $94.255 \%$ \\
\hline 198 & *31:01 & *46:01 & ${ }^{\star} 08: 03$ & $0.098 \%$ & $0.061 \%$ & 0.013 & $94.280 \%$ \\
\hline 198 & *31:01 & *48:01 & ${ }^{\star} 09: 01$ & $0.098 \%$ & $0.062 \%$ & 0.022 & $94.309 \%$ \\
\hline 212 & ${ }^{\star} 02: 01$ & *15:01 & ${ }^{\star} 04: 06$ & $0.092 \%$ & $0.064 \%$ & 0.020 & $94.348 \%$ \\
\hline 212 & *02:01 & *40:02 & ${ }^{*} 04: 05$ & $0.092 \%$ & $-0.035 \%$ & -0.004 & $94.388 \%$ \\
\hline
\end{tabular}




\begin{tabular}{|c|c|c|c|c|c|c|c|}
\hline 212 & ${ }^{\star} 02: 01$ & $* 46: 01$ & $\star^{\star} 09: 01$ & $0.092 \%$ & $0.011 \%$ & 0.002 & $94.413 \%$ \\
\hline 212 & ${ }^{\star} 02: 07$ & $\star 46: 01$ & *04:05 & $0.092 \%$ & $0.069 \%$ & 0.020 & $94.462 \%$ \\
\hline 212 & *24:02 & $\star 51: 01$ & *04:04 & $0.092 \%$ & $0.083 \%$ & 0.325 & $94.491 \%$ \\
\hline 212 & *24:02 & *54:01 & ${ }^{*} 04: 06$ & $0.092 \%$ & $0.005 \%$ & 0.002 & $94.528 \%$ \\
\hline 212 & *24:02 & $\star 55: 02$ & ${ }^{*} 09: 01$ & $0.092 \%$ & $-0.035 \%$ & -0.015 & $94.565 \%$ \\
\hline 212 & *26:01 & *40:02 & *15:01 & $0.092 \%$ & $0.045 \%$ & 0.006 & $94.593 \%$ \\
\hline 212 & *31:01 & *39:01 & *15:01 & $0.092 \%$ & $0.069 \%$ & 0.020 & $94.627 \%$ \\
\hline 212 & *33:03 & *52:01 & *15:02 & $0.092 \%$ & $0.008 \%$ & 0.001 & $94.646 \%$ \\
\hline 222 & ${ }^{*} 02: 01$ & *40:01 & *12:01 & $0.087 \%$ & $0.064 \%$ & 0.018 & $94.684 \%$ \\
\hline 222 & ${ }^{*} 02: 01$ & *40:02 & *14:54 & $0.087 \%$ & $0.054 \%$ & 0.016 & $94.711 \%$ \\
\hline 222 & *02:01 & *51:01 & ${ }^{\star} 04: 03$ & $0.087 \%$ & $0.057 \%$ & 0.021 & $94.760 \%$ \\
\hline 222 & *02:01 & *51:01 & ${ }^{\star} 08: 02$ & $0.087 \%$ & $0.040 \%$ & 0.009 & $94.790 \%$ \\
\hline 222 & *02:06 & *15:01 & *04:06 & $0.087 \%$ & $0.064 \%$ & 0.020 & $94.813 \%$ \\
\hline 222 & ${ }^{*} 02: 06$ & *40:01 & *09:01 & $0.087 \%$ & $0.015 \%$ & 0.003 & $94.843 \%$ \\
\hline 222 & *03:02 & *13:02 & *07:01 & $0.087 \%$ & $0.087 \%$ & 0.883 & $94.899 \%$ \\
\hline 222 & *26:02 & *15:01 & *15:01 & $0.087 \%$ & $0.075 \%$ & 0.041 & $94.916 \%$ \\
\hline 222 & *26:03 & *15:01 & *14:06 & $0.087 \%$ & $0.084 \%$ & 0.066 & $94.954 \%$ \\
\hline 222 & *26:03 & *35:01 & *15:01 & $0.087 \%$ & $0.072 \%$ & 0.032 & $94.979 \%$ \\
\hline 222 & *33:03 & $\star 44: 03$ & ${ }^{\star} 07: 01$ & $0.087 \%$ & $0.085 \%$ & 0.197 & $95.014 \%$ \\
\hline 233 & ${ }^{\star} 02: 01$ & $\star 15: 01$ & *14:06 & $0.081 \%$ & $0.069 \%$ & 0.055 & $95.047 \%$ \\
\hline 233 & ${ }^{\star} 02: 01$ & $\star 35: 01$ & ${ }^{\star} 04: 05$ & $0.081 \%$ & $-0.048 \%$ & -0.006 & $95.074 \%$ \\
\hline 233 & ${ }^{\star} 02: 01$ & *40:01 & *14:05 & $0.081 \%$ & $0.069 \%$ & 0.035 & $95.104 \%$ \\
\hline 233 & ${ }^{\star} 02: 01$ & $\star 40: 02$ & *11:01 & $0.081 \%$ & $0.055 \%$ & 0.020 & $95.134 \%$ \\
\hline 233 & ${ }^{\star} 02: 01$ & $\star 40: 02$ & *14:05 & $0.081 \%$ & $0.062 \%$ & 0.032 & $95.162 \%$ \\
\hline 233 & ${ }^{*} 02: 01$ & *44:03 & *13:02 & $0.081 \%$ & $0.041 \%$ & 0.007 & $95.212 \%$ \\
\hline 233 & ${ }^{*} 02: 01$ & *51:01 & ${ }^{*} 04: 05$ & $0.081 \%$ & $-0.067 \%$ & -0.007 & $95.229 \%$ \\
\hline 233 & ${ }^{*} 02: 01$ & ${ }^{*} 67: 01$ & *12:01 & $0.081 \%$ & $0.076 \%$ & 0.066 & $95.266 \%$ \\
\hline 233 & *02:06 & *48:01 & $\star 15: 01$ & $0.081 \%$ & $0.061 \%$ & 0.022 & $95.302 \%$ \\
\hline 233 & *11:01 & *13:01 & *12:02 & $0.081 \%$ & $0.079 \%$ & 0.065 & $95.336 \%$ \\
\hline 233 & *24:02 & *15:01 & ${ }^{*} 08: 03$ & $0.081 \%$ & $-0.161 \%$ & -0.021 & $95.387 \%$ \\
\hline 233 & *24:02 & *35:01 & *11:01 & $0.081 \%$ & $-0.001 \%$ & 0.000 & $95.415 \%$ \\
\hline 233 & *24:02 & *37:01 & *10:01 & $0.081 \%$ & $0.080 \%$ & 0.139 & $95.467 \%$ \\
\hline 233 & *24:02 & *54:01 & *14:05 & $0.081 \%$ & $0.027 \%$ & 0.014 & $95.506 \%$ \\
\hline 233 & *24:20 & *35:01 & *11:01 & $0.081 \%$ & $0.079 \%$ & 0.117 & $95.533 \%$ \\
\hline 233 & *26:01 & *51:01 & *15:01 & $0.081 \%$ & $0.026 \%$ & 0.003 & $95.563 \%$ \\
\hline 233 & *26:01 & *59:01 & ${ }^{*} 04: 05$ & $0.081 \%$ & $0.061 \%$ & 0.032 & $95.592 \%$ \\
\hline 233 & $\star 26: 03$ & $\star 35: 01$ & ${ }^{\star} 08: 02$ & $0.081 \%$ & $0.073 \%$ & 0.032 & $95.617 \%$ \\
\hline 233 & *31:01 & *15:01 & ${ }^{\star} 09: 01$ & $0.081 \%$ & $-0.018 \%$ & -0.002 & $95.639 \%$ \\
\hline 233 & *31:01 & *35:01 & *15:01 & $0.081 \%$ & $0.025 \%$ & 0.003 & $95.657 \%$ \\
\hline 233 & *31:01 & $\star 39: 02$ & ${ }^{\star} 09: 01$ & $0.081 \%$ & $0.077 \%$ & 0.283 & $95.680 \%$ \\
\hline 233 & *31:01 & *44:03 & *13:02 & $0.081 \%$ & $0.050 \%$ & 0.009 & $95.717 \%$ \\
\hline 233 & *31:01 & *51:01 & ${ }^{*} 08: 03$ & $0.081 \%$ & $0.010 \%$ & 0.001 & $95.754 \%$ \\
\hline 233 & *33:03 & *44:03 & *14:54 & $0.081 \%$ & $0.066 \%$ & 0.019 & $95.790 \%$ \\
\hline 233 & *33:03 & *58:01 & ${ }^{*} 03: 01$ & $0.081 \%$ & $0.081 \%$ & 0.667 & $95.837 \%$ \\
\hline 258 & ${ }^{\star} 02: 01$ & *39:01 & ${ }^{*} 09: 01$ & $0.075 \%$ & $0.018 \%$ & 0.005 & $95.865 \%$ \\
\hline 258 & ${ }^{\star} 02: 01$ & *40:01 & *14:54 & $0.075 \%$ & $0.053 \%$ & 0.015 & $95.882 \%$ \\
\hline 258 & ${ }^{\star} 02: 01$ & *59:01 & ${ }^{*} 04: 05$ & $0.075 \%$ & $0.045 \%$ & 0.024 & $95.898 \%$ \\
\hline 258 & ${ }^{*} 02: 06$ & *51:01 & *14:03 & $0.075 \%$ & $0.061 \%$ & 0.039 & $95.925 \%$ \\
\hline 258 & *24:02 & *15:01 & ${ }^{\star} 04: 05$ & $0.075 \%$ & $-0.306 \%$ & -0.042 & $95.952 \%$ \\
\hline 258 & *24:02 & *39:01 & ${ }^{\star} 09: 01$ & $0.075 \%$ & $-0.103 \%$ & -0.032 & $95.970 \%$ \\
\hline 258 & *24:02 & *48:01 & *14:54 & $0.075 \%$ & $0.040 \%$ & 0.014 & $95.997 \%$ \\
\hline 258 & *24:02 & $\star 51: 01$ & *08:03 & $0.075 \%$ & $-0.214 \%$ & -0.026 & $96.022 \%$ \\
\hline 258 & *26:01 & *35:01 & *12:01 & $0.075 \%$ & $0.053 \%$ & 0.015 & $96.045 \%$ \\
\hline 258 & *31:01 & *35:01 & *04:05 & $0.075 \%$ & $-0.023 \%$ & -0.003 & $96.064 \%$ \\
\hline
\end{tabular}




\begin{tabular}{|c|c|c|c|c|c|c|c|}
\hline 258 & *31:01 & *35:01 & ${ }^{\star} 04: 10$ & $0.075 \%$ & $0.060 \%$ & 0.029 & $96.081 \%$ \\
\hline 258 & *31:01 & *40:01 & ${ }^{*} 09: 01$ & $0.075 \%$ & $0.007 \%$ & 0.001 & $96.102 \%$ \\
\hline 270 & ${ }^{\star} 02: 01$ & *39:04 & ${ }^{\star} 09: 01$ & $0.069 \%$ & $0.066 \%$ & 0.322 & $96.116 \%$ \\
\hline 270 & ${ }^{\star} 02: 01$ & *51:01 & ${ }^{\star} 12: 01$ & $0.069 \%$ & $0.030 \%$ & 0.008 & $96.136 \%$ \\
\hline 270 & ${ }^{*} 02: 06$ & *35:01 & ${ }^{\star} 12: 01$ & $0.069 \%$ & $0.042 \%$ & 0.012 & $96.159 \%$ \\
\hline 270 & ${ }^{*} 02: 06$ & *39:01 & ${ }^{\star} 04: 05$ & $0.069 \%$ & $0.026 \%$ & 0.008 & $96.184 \%$ \\
\hline 270 & ${ }^{*} 02: 06$ & $\star 51: 01$ & ${ }^{*} 08: 03$ & $0.069 \%$ & $-0.005 \%$ & -0.001 & $96.212 \%$ \\
\hline 270 & ${ }^{*} 02: 06$ & *55:02 & ${ }^{*} 09: 01$ & $0.069 \%$ & $0.036 \%$ & 0.015 & $96.238 \%$ \\
\hline 270 & *11:01 & *40:01 & ${ }^{*} 04: 05$ & $0.069 \%$ & $0.004 \%$ & 0.001 & $96.256 \%$ \\
\hline 270 & *11:01 & *48:01 & *15:01 & $0.069 \%$ & $0.050 \%$ & 0.018 & $96.278 \%$ \\
\hline 270 & *11:01 & *51:01 & *15:01 & $0.069 \%$ & $0.004 \%$ & 0.001 & $96.301 \%$ \\
\hline 270 & *24:02 & *48:01 & *16:02 & $0.069 \%$ & $0.059 \%$ & 0.061 & $96.326 \%$ \\
\hline 270 & *24:02 & *51:01 & *14:05 & $0.069 \%$ & $0.003 \%$ & 0.001 & $96.345 \%$ \\
\hline 270 & *24:02 & *52:01 & *11:01 & $0.069 \%$ & $-0.045 \%$ & -0.017 & $96.365 \%$ \\
\hline 270 & *26:01 & *51:01 & *09:01 & $0.069 \%$ & $-0.033 \%$ & -0.004 & $96.392 \%$ \\
\hline 270 & *26:01 & *51:01 & *14:03 & $0.069 \%$ & $0.058 \%$ & 0.036 & $96.411 \%$ \\
\hline 270 & *26:03 & *15:01 & ${ }^{*} 04: 05$ & $0.069 \%$ & $0.045 \%$ & 0.020 & $96.437 \%$ \\
\hline 270 & $\star 26: 03$ & *52:01 & $\star 15: 02$ & $0.069 \%$ & $0.042 \%$ & 0.019 & $96.446 \%$ \\
\hline 270 & *31:01 & *51:01 & ${ }^{\star} 12: 01$ & $0.069 \%$ & $0.039 \%$ & 0.011 & $96.461 \%$ \\
\hline 287 & ${ }^{\star} 02: 01$ & *51:01 & ${ }^{\star} 08: 03$ & $0.063 \%$ & $-0.030 \%$ & -0.003 & $96.472 \%$ \\
\hline 287 & ${ }^{\star} 02: 06$ & *40:02 & ${ }^{\star} 04: 10$ & $0.063 \%$ & $0.048 \%$ & 0.023 & $96.495 \%$ \\
\hline 287 & ${ }^{\star} 02: 06$ & *40:02 & *14:54 & $0.063 \%$ & $0.038 \%$ & 0.011 & $96.510 \%$ \\
\hline 287 & ${ }^{\star} 02: 06$ & *40:06 & ${ }^{\star} 04: 05$ & $0.063 \%$ & $0.006 \%$ & 0.001 & $96.532 \%$ \\
\hline 287 & *11:01 & *51:01 & *11:01 & $0.063 \%$ & $0.040 \%$ & 0.014 & $96.557 \%$ \\
\hline 287 & *11:01 & *55:02 & ${ }^{*} 09: 01$ & $0.063 \%$ & $0.032 \%$ & 0.013 & $96.584 \%$ \\
\hline 287 & *24:02 & *51:01 & ${ }^{*} 04: 03$ & $0.063 \%$ & $-0.030 \%$ & -0.011 & $96.606 \%$ \\
\hline 287 & *24:02 & *52:01 & ${ }^{\star} 01: 01$ & $0.063 \%$ & $-0.174 \%$ & -0.031 & $96.625 \%$ \\
\hline 287 & *24:02 & $\star 54: 01$ & *12:01 & $0.063 \%$ & $-0.035 \%$ & -0.010 & $96.648 \%$ \\
\hline 287 & *24:20 & $\star 55: 02$ & ${ }^{*} 04: 05$ & $0.063 \%$ & $0.061 \%$ & 0.090 & $96.665 \%$ \\
\hline 287 & *26:01 & $\star 54: 01$ & *14:05 & $0.063 \%$ & $0.052 \%$ & 0.026 & $96.692 \%$ \\
\hline 298 & *02:01 & *15:01 & *12:02 & $0.058 \%$ & $0.041 \%$ & 0.023 & $96.716 \%$ \\
\hline 298 & *02:01 & *55:02 & ${ }^{*} 09: 01$ & $0.058 \%$ & $0.017 \%$ & 0.007 & $96.729 \%$ \\
\hline 298 & ${ }^{*} 02: 06$ & *48:01 & *14:05 & $0.058 \%$ & $0.053 \%$ & 0.027 & $96.758 \%$ \\
\hline 298 & ${ }^{*} 02: 06$ & *51:01 & *15:01 & $0.058 \%$ & $-0.009 \%$ & -0.001 & $96.767 \%$ \\
\hline 298 & ${ }^{*} 02: 06$ & *54:01 & *15:01 & $0.058 \%$ & $0.003 \%$ & 0.000 & $96.783 \%$ \\
\hline 298 & ${ }^{*} 02: 07$ & *44:03 & *13:02 & $0.058 \%$ & $0.046 \%$ & 0.013 & $96.801 \%$ \\
\hline 298 & *11:01 & *15:01 & ${ }^{\star} 04: 03$ & $0.058 \%$ & $0.038 \%$ & 0.014 & $96.830 \%$ \\
\hline 298 & *11:01 & *40:06 & ${ }^{\star} 09: 01$ & $0.058 \%$ & $-0.001 \%$ & 0.000 & $96.843 \%$ \\
\hline 298 & *11:01 & *51:01 & ${ }^{\star} 09: 01$ & $0.058 \%$ & $-0.062 \%$ & -0.007 & $96.860 \%$ \\
\hline 298 & $\star 24: 02$ & ${ }^{*} 07: 02$ & ${ }^{\star} 15: 01$ & $0.058 \%$ & $-0.101 \%$ & -0.018 & $96.875 \%$ \\
\hline 298 & $\star 24: 02$ & *39:01 & $\star 15: 01$ & $0.058 \%$ & $-0.039 \%$ & -0.012 & $96.890 \%$ \\
\hline 298 & *24:02 & *51:01 & ${ }^{\star} 15: 02$ & $0.058 \%$ & $-0.300 \%$ & -0.034 & $96.905 \%$ \\
\hline 298 & *24:02 & *52:01 & ${ }^{\star} 04: 03$ & $0.058 \%$ & $-0.056 \%$ & -0.021 & $96.922 \%$ \\
\hline 298 & *24:02 & *54:01 & *15:01 & $0.058 \%$ & $-0.154 \%$ & -0.021 & $96.930 \%$ \\
\hline 298 & *26:01 & *15:01 & ${ }^{\star} 08: 03$ & $0.058 \%$ & $0.007 \%$ & 0.001 & $96.959 \%$ \\
\hline 298 & *26:01 & *15:01 & ${ }^{\star} 09: 01$ & $0.058 \%$ & $-0.028 \%$ & -0.004 & $96.970 \%$ \\
\hline 298 & *26:01 & *37:01 & ${ }^{\star} 10: 01$ & $0.058 \%$ & $0.057 \%$ & 0.100 & $96.996 \%$ \\
\hline 298 & *26:01 & *39:01 & *15:01 & $0.058 \%$ & $0.037 \%$ & 0.011 & $97.006 \%$ \\
\hline 298 & *26:01 & *40:01 & ${ }^{*} 08: 03$ & $0.058 \%$ & $0.023 \%$ & 0.004 & $97.020 \%$ \\
\hline 298 & *26:01 & *40:01 & ${ }^{*} 09: 01$ & $0.058 \%$ & $-0.001 \%$ & 0.000 & $97.031 \%$ \\
\hline 298 & *26:02 & *40:06 & *12:01 & $0.058 \%$ & $0.055 \%$ & 0.029 & $97.053 \%$ \\
\hline 298 & *26:03 & $\star 15: 01$ & *14:54 & $0.058 \%$ & $0.052 \%$ & 0.023 & $97.075 \%$ \\
\hline 298 & *26:03 & $\star 51: 01$ & *14:54 & $0.058 \%$ & $0.050 \%$ & 0.022 & $97.087 \%$ \\
\hline 298 & *31:01 & *35:01 & ${ }^{\star} 08: 02$ & $0.058 \%$ & $0.027 \%$ & 0.006 & $97.098 \%$ \\
\hline
\end{tabular}




\begin{tabular}{|c|c|c|c|c|c|c|c|}
\hline 298 & *31:01 & *39:01 & ${ }^{\star} 08: 03$ & $0.058 \%$ & $0.031 \%$ & 0.009 & $97.105 \%$ \\
\hline 298 & *31:01 & *40:01 & *11:01 & $0.058 \%$ & $0.044 \%$ & 0.016 & $97.124 \%$ \\
\hline 298 & *31:01 & *40:02 & *15:01 & $0.058 \%$ & $0.002 \%$ & 0.000 & $97.132 \%$ \\
\hline 298 & *31:01 & *51:01 & ${ }^{\star} 04: 03$ & $0.058 \%$ & $0.035 \%$ & 0.013 & $97.143 \%$ \\
\hline 298 & *31:01 & *51:01 & *16:02 & $0.058 \%$ & $0.050 \%$ & 0.051 & $97.155 \%$ \\
\hline 298 & *33:03 & *44:03 & ${ }^{\star} 11: 01$ & $0.058 \%$ & $0.046 \%$ & 0.016 & $97.176 \%$ \\
\hline 298 & *33:03 & *44:03 & *15:02 & $0.058 \%$ & $0.011 \%$ & 0.002 & $97.195 \%$ \\
\hline 298 & *33:03 & *54:01 & ${ }^{*} 04: 05$ & $0.058 \%$ & $-0.014 \%$ & -0.002 & $97.203 \%$ \\
\hline 330 & ${ }^{\star} 02: 01$ & $\star 15: 01$ & ${ }^{*} 04: 05$ & $0.052 \%$ & $-0.071 \%$ & -0.009 & $97.218 \%$ \\
\hline 330 & ${ }^{*} 02: 01$ & *15:18 & ${ }^{*} 04: 05$ & $0.052 \%$ & $0.029 \%$ & 0.020 & $97.234 \%$ \\
\hline 330 & ${ }^{\star} 02: 01$ & *35:01 & ${ }^{*} 04: 07$ & $0.052 \%$ & $0.047 \%$ & 0.096 & $97.247 \%$ \\
\hline 330 & ${ }^{*} 02: 01$ & *39:04 & ${ }^{*} 04: 03$ & $0.052 \%$ & $0.051 \%$ & 0.248 & $97.264 \%$ \\
\hline 330 & *02:01 & *40:01 & *11:01 & $0.052 \%$ & $0.035 \%$ & 0.012 & $97.275 \%$ \\
\hline 330 & *02:01 & *48:01 & *09:01 & $0.052 \%$ & $0.005 \%$ & 0.002 & $97.283 \%$ \\
\hline 330 & *02:01 & *56:01 & *09:01 & $0.052 \%$ & $0.037 \%$ & 0.041 & $97.295 \%$ \\
\hline 330 & *02:06 & *13:01 & *12:02 & $0.052 \%$ & $0.050 \%$ & 0.041 & $97.310 \%$ \\
\hline 330 & ${ }^{\star} 02: 06$ & *15:18 & ${ }^{\star} 04: 01$ & $0.052 \%$ & $0.051 \%$ & 0.057 & $97.329 \%$ \\
\hline 330 & ${ }^{\star} 02: 06$ & *39:01 & ${ }^{\star} 09: 01$ & $0.052 \%$ & $0.006 \%$ & 0.002 & $97.339 \%$ \\
\hline 330 & ${ }^{\star} 02: 06$ & *39:01 & *14:54 & $0.052 \%$ & $0.041 \%$ & 0.012 & $97.356 \%$ \\
\hline 330 & ${ }^{\star} 02: 06$ & $* 40: 02$ & ${ }^{\star} 12: 01$ & $0.052 \%$ & $0.025 \%$ & 0.007 & $97.374 \%$ \\
\hline 330 & ${ }^{\star} 02: 06$ & $\star 55: 02$ & ${ }^{\star} 04: 05$ & $0.052 \%$ & $0.021 \%$ & 0.009 & $97.385 \%$ \\
\hline 330 & ${ }^{\star} 02: 06$ & *55:02 & ${ }^{\star} 08: 02$ & $0.052 \%$ & $0.042 \%$ & 0.017 & $97.407 \%$ \\
\hline 330 & ${ }^{\star} 02: 07$ & *15:01 & ${ }^{\star} 04: 06$ & $0.052 \%$ & $0.043 \%$ & 0.014 & $97.414 \%$ \\
\hline 330 & ${ }^{\star} 02: 07$ & *15:01 & *15:01 & $0.052 \%$ & $0.031 \%$ & 0.009 & $97.420 \%$ \\
\hline 330 & *11:01 & *15:01 & ${ }^{*} 08: 03$ & $0.052 \%$ & $-0.008 \%$ & -0.001 & $97.435 \%$ \\
\hline 330 & *11:01 & *39:01 & *15:01 & $0.052 \%$ & $0.028 \%$ & 0.008 & $97.441 \%$ \\
\hline 330 & *11:01 & *40:01 & ${ }^{*} 09: 01$ & $0.052 \%$ & $-0.017 \%$ & -0.003 & $97.450 \%$ \\
\hline 330 & *11:01 & *48:01 & *14:05 & $0.052 \%$ & $0.047 \%$ & 0.024 & $97.468 \%$ \\
\hline 330 & *11:01 & $\star 54: 01$ & ${ }^{\star} 09: 01$ & $0.052 \%$ & $-0.045 \%$ & -0.006 & $97.484 \%$ \\
\hline 330 & *24:02 & *07:02 & ${ }^{\star} 08: 02$ & $0.052 \%$ & $-0.036 \%$ & -0.009 & $97.500 \%$ \\
\hline 330 & *24:02 & *15:01 & ${ }^{*} 04: 03$ & $0.052 \%$ & $-0.026 \%$ & -0.010 & $97.522 \%$ \\
\hline 330 & *24:02 & *15:01 & *12:02 & $0.052 \%$ & $0.001 \%$ & 0.001 & $97.537 \%$ \\
\hline 330 & *24:02 & *15:11 & ${ }^{*} 04: 05$ & $0.052 \%$ & $0.004 \%$ & 0.004 & $97.548 \%$ \\
\hline 330 & *24:02 & *15:18 & ${ }^{*} 04: 10$ & $0.052 \%$ & $0.041 \%$ & 0.029 & $97.566 \%$ \\
\hline 330 & *24:02 & *40:03 & *12:01 & $0.052 \%$ & $0.048 \%$ & 0.141 & $97.581 \%$ \\
\hline 330 & *24:02 & *40:06 & ${ }^{\star} 15: 01$ & $0.052 \%$ & $-0.075 \%$ & -0.017 & $97.593 \%$ \\
\hline 330 & $\star 24: 02$ & *56:01 & ${ }^{\star} 09: 01$ & $0.052 \%$ & $0.004 \%$ & 0.005 & $97.602 \%$ \\
\hline 330 & $\star 26: 01$ & *35:01 & ${ }^{\star} 04: 05$ & $0.052 \%$ & $-0.033 \%$ & -0.004 & $97.609 \%$ \\
\hline 330 & $\star 26: 01$ & *40:01 & ${ }^{\star} 04: 05$ & $0.052 \%$ & $-0.004 \%$ & -0.001 & $97.620 \%$ \\
\hline 330 & $\star 26: 01$ & *40:02 & ${ }^{\star} 04: 03$ & $0.052 \%$ & $0.035 \%$ & 0.013 & $97.640 \%$ \\
\hline 330 & *26:01 & *40:02 & ${ }^{\star} 04: 10$ & $0.052 \%$ & $0.039 \%$ & 0.019 & $97.650 \%$ \\
\hline 330 & *26:02 & ${ }^{*} 07: 02$ & ${ }^{\star} 01: 01$ & $0.052 \%$ & $0.046 \%$ & 0.025 & $97.657 \%$ \\
\hline 330 & *26:02 & *52:01 & $\star 15: 02$ & $0.052 \%$ & $0.030 \%$ & 0.016 & $97.661 \%$ \\
\hline 330 & *31:01 & *15:11 & ${ }^{\star} 09: 01$ & $0.052 \%$ & $0.039 \%$ & 0.041 & $97.670 \%$ \\
\hline 330 & *31:01 & *40:02 & ${ }^{\star} 04: 06$ & $0.052 \%$ & $0.029 \%$ & 0.009 & $97.685 \%$ \\
\hline 367 & ${ }^{\star} 02: 01$ & *15:01 & ${ }^{\star} 08: 03$ & $0.046 \%$ & $-0.032 \%$ & -0.004 & $97.696 \%$ \\
\hline 367 & ${ }^{\star} 02: 01$ & *15:11 & ${ }^{\star} 04: 05$ & $0.046 \%$ & $0.031 \%$ & 0.032 & $97.705 \%$ \\
\hline 367 & ${ }^{\star} 02: 01$ & *40:02 & ${ }^{*} 08: 03$ & $0.046 \%$ & $-0.035 \%$ & -0.004 & $97.716 \%$ \\
\hline 367 & ${ }^{*} 02: 01$ & *55:02 & *12:01 & $0.046 \%$ & $0.036 \%$ & 0.015 & $97.735 \%$ \\
\hline 367 & ${ }^{\star} 02: 06$ & *15:11 & *12:01 & $0.046 \%$ & $0.043 \%$ & 0.044 & $97.750 \%$ \\
\hline 367 & ${ }^{\star} 02: 06$ & *40:01 & ${ }^{\star} 08: 03$ & $0.046 \%$ & $0.003 \%$ & 0.001 & $97.758 \%$ \\
\hline 367 & ${ }^{\star} 02: 06$ & *51:01 & *14:05 & $0.046 \%$ & $0.029 \%$ & 0.015 & $97.769 \%$ \\
\hline 367 & ${ }^{\star} 02: 07$ & *46:01 & *12:01 & $0.046 \%$ & $0.040 \%$ & 0.011 & $97.787 \%$ \\
\hline 367 & ${ }^{\star} 02: 10$ & *40:06 & ${ }^{\star} 09: 01$ & $0.046 \%$ & $0.044 \%$ & 0.107 & $97.792 \%$ \\
\hline
\end{tabular}




\begin{tabular}{|c|c|c|c|c|c|c|c|}
\hline 367 & ${ }^{\star} 02: 10$ & *40:06 & *12:01 & $0.046 \%$ & $0.046 \%$ & 0.111 & $97.804 \%$ \\
\hline 367 & *11:01 & *35:01 & $\star 12: 01$ & $0.046 \%$ & $0.020 \%$ & 0.005 & $97.815 \%$ \\
\hline 367 & *11:01 & *51:01 & ${ }^{*} 08: 02$ & $0.046 \%$ & $0.010 \%$ & 0.002 & $97.828 \%$ \\
\hline 367 & *11:01 & *54:01 & *14:05 & $0.046 \%$ & $0.033 \%$ & 0.017 & $97.839 \%$ \\
\hline 367 & *11:01 & *59:01 & ${ }^{\star} 04: 05$ & $0.046 \%$ & $0.023 \%$ & 0.012 & $97.845 \%$ \\
\hline 367 & *24:02 & ${ }^{*} 07: 02$ & ${ }^{\star} 08: 03$ & $0.046 \%$ & $-0.130 \%$ & -0.024 & $97.855 \%$ \\
\hline 367 & *24:02 & *39:01 & ${ }^{*} 08: 02$ & $0.046 \%$ & $-0.007 \%$ & -0.002 & $97.873 \%$ \\
\hline 367 & *24:02 & *40:01 & ${ }^{*} 08: 02$ & $0.046 \%$ & $-0.036 \%$ & -0.009 & $97.885 \%$ \\
\hline 367 & *24:02 & *40:02 & ${ }^{*} 04: 06$ & $0.046 \%$ & $-0.047 \%$ & -0.015 & $97.896 \%$ \\
\hline 367 & *24:02 & *46:01 & *04:05 & $0.046 \%$ & $-0.193 \%$ & -0.042 & $97.903 \%$ \\
\hline 367 & *24:02 & $\star 51: 01$ & *04:10 & $0.046 \%$ & $-0.025 \%$ & -0.012 & $97.913 \%$ \\
\hline 367 & *24:02 & *52:01 & *12:02 & $0.046 \%$ & $-0.028 \%$ & -0.016 & $97.922 \%$ \\
\hline 367 & *24:02 & *55:02 & *08:02 & $0.046 \%$ & $0.008 \%$ & 0.003 & $97.935 \%$ \\
\hline 367 & *24:02 & *58:01 & *13:02 & $0.046 \%$ & $0.034 \%$ & 0.057 & $97.950 \%$ \\
\hline 367 & *24:20 & *35:01 & *04:05 & $0.046 \%$ & $0.039 \%$ & 0.057 & $97.955 \%$ \\
\hline 367 & *26:01 & *15:07 & ${ }^{*} 04: 03$ & $0.046 \%$ & $0.045 \%$ & 0.076 & $97.970 \%$ \\
\hline 367 & *26:01 & *35:01 & *11:01 & $0.046 \%$ & $0.029 \%$ & 0.010 & $97.979 \%$ \\
\hline 367 & $\star 26: 01$ & *39:01 & ${ }^{\star} 08: 02$ & $0.046 \%$ & $0.035 \%$ & 0.010 & $97.991 \%$ \\
\hline 367 & $\star 26: 01$ & *40:01 & *11:01 & $0.046 \%$ & $0.035 \%$ & 0.012 & $98.000 \%$ \\
\hline 367 & $\star 26: 01$ & *40:02 & *11:01 & $0.046 \%$ & $0.029 \%$ & 0.010 & $98.007 \%$ \\
\hline 367 & *26:01 & *40:06 & ${ }^{\star} 04: 05$ & $0.046 \%$ & $-0.001 \%$ & 0.000 & $98.017 \%$ \\
\hline 367 & *26:01 & *48:01 & *15:01 & $0.046 \%$ & $0.029 \%$ & 0.011 & $98.026 \%$ \\
\hline 367 & *26:01 & *56:01 & ${ }^{\star} 09: 01$ & $0.046 \%$ & $0.036 \%$ & 0.040 & $98.034 \%$ \\
\hline 367 & *26:02 & *15:01 & ${ }^{\star} 09: 01$ & $0.046 \%$ & $0.026 \%$ & 0.014 & $98.039 \%$ \\
\hline 367 & *26:03 & *51:01 & *14:03 & $0.046 \%$ & $0.043 \%$ & 0.027 & $98.046 \%$ \\
\hline 367 & *31:01 & *51:01 & $\star 12: 02$ & $0.046 \%$ & $0.031 \%$ & 0.017 & $98.054 \%$ \\
\hline 367 & *31:01 & $\star 51: 02$ & ${ }^{*} 09: 01$ & $0.046 \%$ & $0.044 \%$ & 0.226 & $98.061 \%$ \\
\hline 367 & *31:01 & $\star 59: 01$ & ${ }^{\star} 04: 05$ & $0.046 \%$ & $0.023 \%$ & 0.012 & $98.065 \%$ \\
\hline 367 & *33:03 & ${ }^{\star} 07: 02$ & *01:01 & $0.046 \%$ & $0.023 \%$ & 0.004 & $98.071 \%$ \\
\hline 367 & *33:03 & *44:03 & *12:01 & $0.046 \%$ & $0.030 \%$ & 0.008 & $98.084 \%$ \\
\hline 367 & *33:03 & *44:03 & *14:05 & $0.046 \%$ & $0.038 \%$ & 0.019 & $98.094 \%$ \\
\hline 407 & ${ }^{*} 02: 01$ & *15:01 & ${ }^{\star} 08: 02$ & $0.040 \%$ & $0.002 \%$ & 0.000 & $98.105 \%$ \\
\hline 407 & ${ }^{\star} 02: 01$ & *15:18 & *08:03 & $0.040 \%$ & $0.026 \%$ & 0.018 & $98.116 \%$ \\
\hline 407 & ${ }^{\star} 02: 01$ & *35:01 & *08:03 & $0.040 \%$ & $-0.041 \%$ & -0.005 & $98.125 \%$ \\
\hline 407 & ${ }^{\star} 02: 01$ & *39:01 & ${ }^{\star} 04: 10$ & $0.040 \%$ & $0.032 \%$ & 0.015 & $98.137 \%$ \\
\hline 407 & ${ }^{\star} 02: 01$ & $* 40: 02$ & *15:02 & $0.040 \%$ & $-0.059 \%$ & -0.007 & $98.147 \%$ \\
\hline 407 & ${ }^{\star} 02: 01$ & *48:01 & $\star 12: 01$ & $0.040 \%$ & $0.029 \%$ & 0.010 & $98.159 \%$ \\
\hline 407 & ${ }^{\star} 02: 01$ & *55:02 & $\star 15: 01$ & $0.040 \%$ & $0.018 \%$ & 0.007 & $98.169 \%$ \\
\hline 407 & ${ }^{\star} 02: 01$ & *55:04 & ${ }^{\star} 09: 01$ & $0.040 \%$ & $0.038 \%$ & 0.293 & $98.174 \%$ \\
\hline 407 & ${ }^{\star} 02: 06$ & *15:01 & $\star 14: 06$ & $0.040 \%$ & $0.031 \%$ & 0.025 & $98.186 \%$ \\
\hline 407 & ${ }^{\star} 02: 06$ & *35:01 & $\star 14: 54$ & $0.040 \%$ & $0.014 \%$ & 0.004 & $98.195 \%$ \\
\hline 407 & ${ }^{\star} 02: 06$ & *40:06 & *12:01 & $0.040 \%$ & $0.025 \%$ & 0.007 & $98.205 \%$ \\
\hline 407 & ${ }^{\star} 02: 06$ & *40:06 & *14:54 & $0.040 \%$ & $0.026 \%$ & 0.007 & $98.218 \%$ \\
\hline 407 & ${ }^{\star} 02: 06$ & *44:03 & *13:02 & $0.040 \%$ & $0.008 \%$ & 0.002 & $98.229 \%$ \\
\hline 407 & ${ }^{\star} 02: 06$ & *48:01 & ${ }^{*} 04: 05$ & $0.040 \%$ & $0.005 \%$ & 0.002 & $98.238 \%$ \\
\hline 407 & ${ }^{\star} 02: 06$ & *51:01 & *16:02 & $0.040 \%$ & $0.032 \%$ & 0.033 & $98.247 \%$ \\
\hline 407 & ${ }^{\star} 02: 07$ & ${ }^{*} 07: 02$ & ${ }^{*} 01: 01$ & $0.040 \%$ & $0.029 \%$ & 0.008 & $98.250 \%$ \\
\hline 407 & ${ }^{\star} 02: 07$ & $\star 52: 01$ & *15:02 & $0.040 \%$ & $-0.002 \%$ & 0.000 & $98.252 \%$ \\
\hline 407 & ${ }^{\star} 02: 18$ & *46:01 & ${ }^{*} 08: 03$ & $0.040 \%$ & $0.040 \%$ & 0.874 & $98.255 \%$ \\
\hline 407 & *11:01 & *15:18 & *09:01 & $0.040 \%$ & $0.022 \%$ & 0.015 & $98.266 \%$ \\
\hline 407 & *11:01 & *35:01 & *09:01 & $0.040 \%$ & $-0.064 \%$ & -0.008 & $98.273 \%$ \\
\hline 407 & *11:01 & *35:01 & *15:01 & $0.040 \%$ & $-0.016 \%$ & -0.002 & $98.278 \%$ \\
\hline 407 & *11:01 & *40:01 & *14:54 & $0.040 \%$ & $0.024 \%$ & 0.007 & $98.289 \%$ \\
\hline 407 & *11:01 & *40:02 & *08:02 & $0.040 \%$ & $0.010 \%$ & 0.002 & $98.296 \%$ \\
\hline
\end{tabular}




\begin{tabular}{|c|c|c|c|c|c|c|c|}
\hline 407 & *11:01 & *44:02 & $\star 13: 01$ & $0.040 \%$ & $0.040 \%$ & 0.106 & $98.311 \%$ \\
\hline 407 & *11:01 & *52:01 & *09:01 & $0.040 \%$ & $-0.105 \%$ & -0.012 & $98.316 \%$ \\
\hline 407 & *24:02 & *07:02 & *12:01 & $0.040 \%$ & $-0.034 \%$ & -0.010 & $98.323 \%$ \\
\hline 407 & *24:02 & $\star 07: 02$ & *15:02 & $0.040 \%$ & $-0.178 \%$ & -0.033 & $98.331 \%$ \\
\hline 407 & *24:02 & *35:01 & *08:03 & $0.040 \%$ & $-0.212 \%$ & -0.027 & $98.340 \%$ \\
\hline 407 & *24:02 & *35:01 & *14:06 & $0.040 \%$ & $0.003 \%$ & 0.003 & $98.350 \%$ \\
\hline 407 & *24:02 & *40:01 & ${ }^{\star} 01: 01$ & $0.040 \%$ & $-0.072 \%$ & -0.014 & $98.358 \%$ \\
\hline 407 & *24:02 & *40:01 & ${ }^{\star} 04: 03$ & $0.040 \%$ & $-0.013 \%$ & -0.005 & $98.366 \%$ \\
\hline 407 & *24:02 & *40:02 & ${ }^{\star} 04: 03$ & $0.040 \%$ & $-0.040 \%$ & -0.015 & $98.373 \%$ \\
\hline 407 & *24:02 & *40:06 & *11:01 & $0.040 \%$ & $-0.006 \%$ & -0.002 & $98.383 \%$ \\
\hline 407 & *24:02 & *40:06 & *14:54 & $0.040 \%$ & $-0.017 \%$ & -0.005 & $98.392 \%$ \\
\hline 407 & *24:02 & *52:01 & ${ }^{*} 08: 02$ & $0.040 \%$ & $-0.134 \%$ & -0.033 & $98.399 \%$ \\
\hline 407 & *24:02 & *52:01 & *12:01 & $0.040 \%$ & $-0.108 \%$ & -0.031 & $98.404 \%$ \\
\hline 407 & *24:20 & *40:01 & *08:03 & $0.040 \%$ & $0.037 \%$ & 0.055 & $98.409 \%$ \\
\hline 407 & *26:01 & *15:01 & *15:01 & $0.040 \%$ & $-0.006 \%$ & -0.001 & $98.411 \%$ \\
\hline 407 & *26:01 & *35:01 & ${ }^{\star} 04: 06$ & $0.040 \%$ & $0.021 \%$ & 0.006 & $98.419 \%$ \\
\hline 407 & *26:01 & *40:02 & *12:01 & $0.040 \%$ & $0.018 \%$ & 0.005 & $98.426 \%$ \\
\hline 407 & *26:01 & *48:01 & *14:07 & $0.040 \%$ & $0.040 \%$ & 0.436 & $98.437 \%$ \\
\hline 407 & *26:01 & *51:01 & ${ }^{\star} 04: 05$ & $0.040 \%$ & $-0.057 \%$ & -0.007 & $98.442 \%$ \\
\hline 407 & *26:01 & $\star 56: 01$ & *11:01 & $0.040 \%$ & $0.038 \%$ & 0.042 & $98.454 \%$ \\
\hline 407 & *26:03 & $\star 54: 01$ & ${ }^{\star} 04: 05$ & $0.040 \%$ & $0.017 \%$ & 0.008 & $98.457 \%$ \\
\hline 407 & *31:01 & *15:01 & $\star 08: 03$ & $0.040 \%$ & $-0.019 \%$ & -0.002 & $98.466 \%$ \\
\hline 407 & *31:01 & $\star 35: 01$ & *12:01 & $0.040 \%$ & $0.014 \%$ & 0.004 & $98.471 \%$ \\
\hline 407 & *31:01 & *40:01 & *04:05 & $0.040 \%$ & $-0.024 \%$ & -0.005 & $98.477 \%$ \\
\hline 407 & *31:01 & *40:01 & *14:54 & $0.040 \%$ & $0.024 \%$ & 0.007 & $98.483 \%$ \\
\hline 455 & ${ }^{\star} 02: 01$ & *13:01 & ${ }^{\star} 04: 05$ & $0.035 \%$ & $0.015 \%$ & 0.013 & $98.489 \%$ \\
\hline 455 & ${ }^{\star} 02: 01$ & *35:01 & *11:01 & $0.035 \%$ & $0.008 \%$ & 0.003 & $98.493 \%$ \\
\hline 455 & ${ }^{\star} 02: 01$ & *39:02 & *14:54 & $0.035 \%$ & $0.034 \%$ & 0.121 & $98.500 \%$ \\
\hline 455 & ${ }^{\star} 02: 01$ & *40:03 & *14:05 & $0.035 \%$ & $0.034 \%$ & 0.099 & $98.512 \%$ \\
\hline 455 & ${ }^{\star} 02: 01$ & *40:06 & *15:01 & $0.035 \%$ & $-0.006 \%$ & -0.001 & $98.520 \%$ \\
\hline 455 & ${ }^{\star} 02: 01$ & *48:01 & *11:01 & $0.035 \%$ & $0.026 \%$ & 0.009 & $98.527 \%$ \\
\hline 455 & ${ }^{\star} 02: 01$ & *54:01 & *12:01 & $0.035 \%$ & $0.003 \%$ & 0.001 & $98.533 \%$ \\
\hline 455 & ${ }^{*} 02: 01$ & *59:01 & *09:01 & $0.035 \%$ & $0.003 \%$ & 0.001 & $98.537 \%$ \\
\hline 455 & ${ }^{*} 02: 01$ & *67:01 & *16:02 & $0.035 \%$ & $0.033 \%$ & 0.034 & $98.545 \%$ \\
\hline 455 & ${ }^{*} 02: 06$ & *15:01 & *08:03 & $0.035 \%$ & $-0.028 \%$ & -0.004 & $98.551 \%$ \\
\hline 455 & ${ }^{\star} 02: 06$ & *15:11 & ${ }^{\star} 09: 01$ & $0.035 \%$ & $0.022 \%$ & 0.022 & $98.555 \%$ \\
\hline 455 & ${ }^{\star} 02: 06$ & *15:18 & $\star 04: 05$ & $0.035 \%$ & $0.016 \%$ & 0.012 & $98.561 \%$ \\
\hline 455 & ${ }^{\star} 02: 06$ & *15:18 & *12:01 & $0.035 \%$ & $0.030 \%$ & 0.021 & $98.569 \%$ \\
\hline 455 & ${ }^{\star} 02: 06$ & *39:02 & ${ }^{\star} 09: 01$ & $0.035 \%$ & $0.031 \%$ & 0.113 & $98.572 \%$ \\
\hline 455 & ${ }^{\star} 02: 06$ & *40:01 & *12:01 & $0.035 \%$ & $0.017 \%$ & 0.005 & $98.578 \%$ \\
\hline 455 & ${ }^{\star} 02: 06$ & *40:01 & *15:01 & $0.035 \%$ & $-0.004 \%$ & -0.001 & $98.582 \%$ \\
\hline 455 & ${ }^{\star} 02: 06$ & *40:06 & *15:01 & $0.035 \%$ & $0.002 \%$ & 0.000 & $98.587 \%$ \\
\hline 455 & *02:06 & *48:01 & *14:54 & $0.035 \%$ & $0.026 \%$ & 0.009 & $98.593 \%$ \\
\hline 455 & *02:06 & $\star 51: 01$ & *14:54 & $0.035 \%$ & $0.005 \%$ & 0.001 & $98.597 \%$ \\
\hline 455 & ${ }^{\star} 02: 06$ & *55:02 & *08:03 & $0.035 \%$ & $0.015 \%$ & 0.006 & $98.607 \%$ \\
\hline 455 & ${ }^{\star} 02: 06$ & *55:02 & *15:01 & $0.035 \%$ & $0.017 \%$ & 0.007 & $98.611 \%$ \\
\hline 455 & ${ }^{\star} 02: 07$ & *15:01 & *04:05 & $0.035 \%$ & $-0.002 \%$ & -0.001 & $98.616 \%$ \\
\hline 455 & *11:01 & *15:11 & *09:01 & $0.035 \%$ & $0.022 \%$ & 0.023 & $98.619 \%$ \\
\hline 455 & *11:01 & *40:01 & *15:01 & $0.035 \%$ & $-0.003 \%$ & 0.000 & $98.623 \%$ \\
\hline 455 & *11:01 & $\star 44: 03$ & *13:02 & $0.035 \%$ & $0.004 \%$ & 0.001 & $98.629 \%$ \\
\hline 455 & *11:01 & *46:01 & ${ }^{\star} 09: 01$ & $0.035 \%$ & $-0.028 \%$ & -0.006 & $98.633 \%$ \\
\hline 455 & *11:01 & *51:01 & *12:01 & $0.035 \%$ & $0.004 \%$ & 0.001 & $98.638 \%$ \\
\hline 455 & *11:01 & *51:01 & *14:03 & $0.035 \%$ & $0.021 \%$ & 0.013 & $98.641 \%$ \\
\hline 455 & *24:02 & *15:11 & ${ }^{\star} 09: 01$ & $0.035 \%$ & $-0.016 \%$ & -0.017 & $98.644 \%$ \\
\hline
\end{tabular}




\begin{tabular}{|c|c|c|c|c|c|c|c|}
\hline 455 & $\star 24: 02$ & $\star 15: 18$ & ${ }^{\star} 04: 03$ & $0.035 \%$ & $0.020 \%$ & 0.014 & $98.653 \%$ \\
\hline 455 & $\star 24: 02$ & *15:18 & $\star^{\star} 08: 03$ & $0.035 \%$ & $-0.010 \%$ & -0.007 & $98.658 \%$ \\
\hline 455 & $\star 24: 02$ & *15:18 & $\star^{\star} 09: 01$ & $0.035 \%$ & $-0.040 \%$ & -0.029 & $98.662 \%$ \\
\hline 455 & *24:02 & *39:01 & ${ }^{*} 04: 05$ & $0.035 \%$ & $-0.134 \%$ & -0.041 & $98.666 \%$ \\
\hline 455 & *24:02 & *40:01 & ${ }^{\star} 04: 06$ & $0.035 \%$ & $-0.027 \%$ & -0.009 & $98.672 \%$ \\
\hline 455 & *24:02 & *40:06 & ${ }^{*} 04: 10$ & $0.035 \%$ & $0.000 \%$ & 0.000 & $98.679 \%$ \\
\hline 455 & *24:02 & *48:01 & *11:01 & $0.035 \%$ & $0.006 \%$ & 0.002 & $98.686 \%$ \\
\hline 455 & *24:02 & *48:01 & *12:01 & $0.035 \%$ & $-0.002 \%$ & -0.001 & $98.692 \%$ \\
\hline 455 & *24:02 & *52:01 & ${ }^{\star} 04: 06$ & $0.035 \%$ & $-0.096 \%$ & -0.031 & $98.696 \%$ \\
\hline 455 & *24:02 & *52:01 & *14:54 & $0.035 \%$ & $-0.107 \%$ & -0.032 & $98.700 \%$ \\
\hline 455 & *24:02 & *54:01 & ${ }^{\star} 04: 03$ & $0.035 \%$ & $-0.041 \%$ & -0.015 & $98.705 \%$ \\
\hline 455 & *24:02 & *54:01 & ${ }^{\star} 08: 02$ & $0.035 \%$ & $-0.082 \%$ & -0.020 & $98.712 \%$ \\
\hline 455 & *24:02 & *54:01 & *14:03 & $0.035 \%$ & $-0.009 \%$ & -0.006 & $98.718 \%$ \\
\hline 455 & *24:02 & $\star 54: 01$ & *15:02 & $0.035 \%$ & $-0.256 \%$ & -0.035 & $98.724 \%$ \\
\hline 455 & *24:02 & *55:02 & *14:54 & $0.035 \%$ & $0.004 \%$ & 0.002 & $98.732 \%$ \\
\hline 455 & *24:02 & $\star 56: 01$ & ${ }^{*} 04: 05$ & $0.035 \%$ & $-0.011 \%$ & -0.012 & $98.736 \%$ \\
\hline 455 & *24:20 & *46:01 & ${ }^{*} 08: 03$ & $0.035 \%$ & $0.032 \%$ & 0.047 & $98.739 \%$ \\
\hline 455 & $\star 26: 01$ & $\star 40: 01$ & *14:05 & $0.035 \%$ & $0.027 \%$ & 0.013 & $98.746 \%$ \\
\hline 455 & *26:01 & *48:01 & ${ }^{\star} 09: 01$ & $0.035 \%$ & $0.004 \%$ & 0.001 & $98.749 \%$ \\
\hline 455 & *26:01 & $\star 51: 01$ & ${ }^{\star} 08: 02$ & $0.035 \%$ & $0.004 \%$ & 0.001 & $98.753 \%$ \\
\hline 455 & *26:01 & $\star 54: 01$ & ${ }^{\star} 09: 01$ & $0.035 \%$ & $-0.048 \%$ & -0.006 & $98.757 \%$ \\
\hline 455 & *26:02 & $\star 35: 01$ & *15:01 & $0.035 \%$ & $0.023 \%$ & 0.012 & $98.760 \%$ \\
\hline 455 & *26:03 & $\star 15: 01$ & ${ }^{\star} 08: 02$ & $0.035 \%$ & $0.027 \%$ & 0.012 & $98.767 \%$ \\
\hline 455 & *26:03 & *15:01 & ${ }^{\star} 12: 01$ & $0.035 \%$ & $0.028 \%$ & 0.012 & $98.773 \%$ \\
\hline 455 & *26:03 & *46:01 & ${ }^{*} 08: 03$ & $0.035 \%$ & $0.025 \%$ & 0.011 & $98.776 \%$ \\
\hline 455 & *31:01 & *15:01 & ${ }^{*} 04: 05$ & $0.035 \%$ & $-0.059 \%$ & -0.008 & $98.782 \%$ \\
\hline 455 & *31:01 & *15:01 & ${ }^{\star} 08: 02$ & $0.035 \%$ & $0.005 \%$ & 0.001 & $98.790 \%$ \\
\hline 455 & *31:01 & *15:01 & *11:01 & $0.035 \%$ & $0.015 \%$ & 0.006 & $98.796 \%$ \\
\hline 455 & *31:01 & *15:07 & ${ }^{*} 04: 05$ & $0.035 \%$ & $0.028 \%$ & 0.047 & $98.801 \%$ \\
\hline 455 & *31:01 & *15:18 & ${ }^{\star} 09: 01$ & $0.035 \%$ & $0.016 \%$ & 0.011 & $98.805 \%$ \\
\hline 455 & *31:01 & *40:01 & *14:05 & $0.035 \%$ & $0.025 \%$ & 0.013 & $98.810 \%$ \\
\hline 455 & *31:01 & *40:02 & ${ }^{*} 08: 03$ & $0.035 \%$ & $-0.027 \%$ & -0.003 & $98.816 \%$ \\
\hline 455 & $\star 31: 01$ & *40:02 & *14:05 & $0.035 \%$ & $0.021 \%$ & 0.010 & $98.821 \%$ \\
\hline 455 & $\star 31: 01$ & *51:01 & ${ }^{*} 04: 01$ & $0.035 \%$ & $0.027 \%$ & 0.031 & $98.826 \%$ \\
\hline 455 & $\star 31: 01$ & *56:01 & ${ }^{*} 04: 05$ & $0.035 \%$ & $0.024 \%$ & 0.026 & $98.831 \%$ \\
\hline 455 & $\star 33: 03$ & *44:03 & ${ }^{*} 08: 02$ & $0.035 \%$ & $0.016 \%$ & 0.004 & $98.837 \%$ \\
\hline 455 & *33:03 & *46:01 & ${ }^{\star} 08: 03$ & $0.035 \%$ & $0.005 \%$ & 0.001 & $98.838 \%$ \\
\hline 521 & ${ }^{\star} 02: 01$ & *15:11 & ${ }^{*} 01: 01$ & $0.029 \%$ & $0.022 \%$ & 0.023 & $98.846 \%$ \\
\hline 521 & ${ }^{\star} 02: 01$ & *15:11 & *15:01 & $0.029 \%$ & $0.020 \%$ & 0.021 & $98.850 \%$ \\
\hline 521 & ${ }^{\star} 02: 01$ & *15:18 & ${ }^{\star} 08: 02$ & $0.029 \%$ & $0.022 \%$ & 0.015 & $98.855 \%$ \\
\hline 521 & $\star 02: 01$ & *27:05 & ${ }^{\star} 01: 01$ & $0.029 \%$ & $0.028 \%$ & 0.353 & $98.863 \%$ \\
\hline 521 & $\star 02: 01$ & *37:01 & *10:01 & $0.029 \%$ & $0.028 \%$ & 0.050 & $98.871 \%$ \\
\hline 521 & ${ }^{\star} 02: 01$ & *40:02 & ${ }^{*} 04: 03$ & $0.029 \%$ & $0.003 \%$ & 0.001 & $98.875 \%$ \\
\hline 521 & ${ }^{\star} 02: 01$ & *40:02 & ${ }^{*} 04: 10$ & $0.029 \%$ & $0.009 \%$ & 0.004 & $98.877 \%$ \\
\hline 521 & ${ }^{\star} 02: 01$ & *48:01 & *15:01 & $0.029 \%$ & $0.003 \%$ & 0.001 & $98.880 \%$ \\
\hline 521 & ${ }^{\star} 02: 01$ & *51:01 & ${ }^{*} 04: 06$ & $0.029 \%$ & $-0.006 \%$ & -0.002 & $98.887 \%$ \\
\hline 521 & *02:01 & *51:01 & *12:02 & $0.029 \%$ & $0.009 \%$ & 0.005 & $98.891 \%$ \\
\hline 521 & *02:01 & *52:01 & ${ }^{*} 09: 01$ & $0.029 \%$ & $-0.160 \%$ & -0.014 & $98.892 \%$ \\
\hline 521 & *02:01 & *55:02 & *11:01 & $0.029 \%$ & $0.021 \%$ & 0.008 & $98.898 \%$ \\
\hline 521 & *02:01 & *56:03 & *12:01 & $0.029 \%$ & $0.028 \%$ & 0.124 & $98.902 \%$ \\
\hline 521 & *02:06 & *15:01 & ${ }^{\star} 04: 03$ & $0.029 \%$ & $0.009 \%$ & 0.003 & $98.909 \%$ \\
\hline 521 & *02:06 & *15:11 & ${ }^{\star} 04: 03$ & $0.029 \%$ & $0.026 \%$ & 0.027 & $98.915 \%$ \\
\hline 521 & ${ }^{\star} 02: 06$ & *35:01 & ${ }^{\star} 08: 02$ & $0.029 \%$ & $-0.003 \%$ & -0.001 & $98.919 \%$ \\
\hline 521 & *02:06 & *35:01 & *08:03 & $0.029 \%$ & $-0.036 \%$ & -0.004 & $98.923 \%$ \\
\hline
\end{tabular}




\begin{tabular}{|c|c|c|c|c|c|c|c|}
\hline 521 & ${ }^{*} 02: 06$ & *37:01 & *10:01 & $0.029 \%$ & $0.029 \%$ & 0.050 & $98.929 \%$ \\
\hline 521 & ${ }^{\star} 02: 06$ & *39:01 & $\star 04: 03$ & $0.029 \%$ & $0.020 \%$ & 0.007 & $98.935 \%$ \\
\hline 521 & ${ }^{\star} 02: 06$ & *39:01 & *14:06 & $0.029 \%$ & $0.025 \%$ & 0.020 & $98.941 \%$ \\
\hline 521 & *02:06 & *40:01 & *14:54 & $0.029 \%$ & $0.012 \%$ & 0.003 & $98.943 \%$ \\
\hline 521 & *02:06 & *40:02 & *04:05 & $0.029 \%$ & $-0.073 \%$ & -0.009 & $98.946 \%$ \\
\hline 521 & *02:06 & *40:02 & *11:01 & $0.029 \%$ & $0.008 \%$ & 0.003 & $98.950 \%$ \\
\hline 521 & ${ }^{\star} 02: 06$ & *40:02 & *14:05 & $0.029 \%$ & $0.014 \%$ & 0.007 & $98.953 \%$ \\
\hline 521 & ${ }^{\star} 02: 06$ & *46:01 & *09:01 & $0.029 \%$ & $-0.036 \%$ & -0.007 & $98.955 \%$ \\
\hline 521 & ${ }^{\star} 02: 06$ & *51:01 & ${ }^{\star} 08: 02$ & $0.029 \%$ & $-0.008 \%$ & -0.002 & $98.957 \%$ \\
\hline 521 & ${ }^{\star} 02: 06$ & *54:01 & *08:03 & $0.029 \%$ & $-0.032 \%$ & -0.004 & $98.960 \%$ \\
\hline 521 & ${ }^{\star} 02: 06$ & $\star 54: 01$ & *11:01 & $0.029 \%$ & $0.009 \%$ & 0.003 & $98.965 \%$ \\
\hline 521 & ${ }^{\star} 02: 06$ & *55:02 & *14:54 & $0.029 \%$ & $0.021 \%$ & 0.009 & $98.968 \%$ \\
\hline 521 & ${ }^{\star} 02: 07$ & *46:01 & *15:01 & $0.029 \%$ & $0.016 \%$ & 0.005 & $98.975 \%$ \\
\hline 521 & *03:01 & *52:01 & *15:02 & $0.029 \%$ & $0.024 \%$ & 0.060 & $98.976 \%$ \\
\hline 521 & *11:01 & *40:01 & *11:01 & $0.029 \%$ & $0.015 \%$ & 0.006 & $98.979 \%$ \\
\hline 521 & *11:01 & *40:01 & $\star 12: 02$ & $0.029 \%$ & $0.020 \%$ & 0.011 & $98.985 \%$ \\
\hline 521 & *11:01 & $* 40: 02$ & ${ }^{\star} 04: 05$ & $0.029 \%$ & $-0.070 \%$ & -0.009 & $98.989 \%$ \\
\hline 521 & *11:01 & *40:02 & ${ }^{\star} 09: 01$ & $0.029 \%$ & $-0.075 \%$ & -0.009 & $98.992 \%$ \\
\hline 521 & *11:01 & *40:02 & *11:01 & $0.029 \%$ & $0.009 \%$ & 0.003 & $98.994 \%$ \\
\hline 521 & *11:01 & *48:01 & *04:05 & $0.029 \%$ & $-0.006 \%$ & -0.002 & $98.998 \%$ \\
\hline 521 & *11:01 & *51:01 & *04:04 & $0.029 \%$ & $0.027 \%$ & 0.101 & $99.001 \%$ \\
\hline 521 & *11:01 & *51:01 & *04:06 & $0.029 \%$ & $0.002 \%$ & 0.001 & $99.007 \%$ \\
\hline 521 & *11:01 & *54:01 & *08:02 & $0.029 \%$ & $0.000 \%$ & 0.000 & $99.012 \%$ \\
\hline 521 & *11:01 & *55:02 & *12:01 & $0.029 \%$ & $0.021 \%$ & 0.008 & $99.019 \%$ \\
\hline 521 & *11:01 & *55:04 & *09:01 & $0.029 \%$ & $0.027 \%$ & 0.207 & $99.021 \%$ \\
\hline 521 & *24:02 & *15:01 & *08:02 & $0.029 \%$ & $-0.091 \%$ & -0.022 & $99.025 \%$ \\
\hline 521 & *24:02 & *40:02 & *04:01 & $0.029 \%$ & $0.003 \%$ & 0.003 & $99.029 \%$ \\
\hline 521 & *24:02 & *48:01 & *04:03 & $0.029 \%$ & $0.001 \%$ & 0.000 & $99.034 \%$ \\
\hline 521 & *24:02 & *48:01 & *04:07 & $0.029 \%$ & $0.024 \%$ & 0.048 & $99.039 \%$ \\
\hline 521 & *24:02 & *48:01 & *04:10 & $0.029 \%$ & $0.007 \%$ & 0.004 & $99.044 \%$ \\
\hline 521 & *24:02 & *51:02 & *14:54 & $0.029 \%$ & $0.026 \%$ & 0.136 & $99.047 \%$ \\
\hline 521 & *24:02 & $\star 52: 01$ & *14:05 & $0.029 \%$ & $-0.052 \%$ & -0.027 & $99.050 \%$ \\
\hline 521 & *24:02 & *55:02 & ${ }^{\star} 04: 06$ & $0.029 \%$ & $0.001 \%$ & 0.000 & $99.056 \%$ \\
\hline 521 & *24:02 & *55:02 & *11:01 & $0.029 \%$ & $0.004 \%$ & 0.002 & $99.061 \%$ \\
\hline 521 & *24:02 & *55:02 & *12:01 & $0.029 \%$ & $-0.003 \%$ & -0.001 & $99.064 \%$ \\
\hline 521 & *24:02 & *59:01 & *09:01 & $0.029 \%$ & $-0.070 \%$ & -0.039 & $99.066 \%$ \\
\hline 521 & $\star 24: 20$ & $* 40: 01$ & ${ }^{\star} 04: 05$ & $0.029 \%$ & $0.024 \%$ & 0.035 & $99.069 \%$ \\
\hline 521 & *24:20 & *40:02 & *09:01 & $0.029 \%$ & $0.021 \%$ & 0.031 & $99.072 \%$ \\
\hline 521 & *24:20 & *55:02 & *09:01 & $0.029 \%$ & $0.027 \%$ & 0.039 & $99.075 \%$ \\
\hline 521 & *26:01 & *15:01 & ${ }^{*} 04: 05$ & $0.029 \%$ & $-0.052 \%$ & -0.007 & $99.078 \%$ \\
\hline 521 & *26:01 & *15:01 & *12:02 & $0.029 \%$ & $0.018 \%$ & 0.010 & $99.083 \%$ \\
\hline 521 & *26:01 & *15:01 & *14:54 & $0.029 \%$ & $0.008 \%$ & 0.002 & $99.090 \%$ \\
\hline 521 & *26:01 & $\star 40: 01$ & *14:06 & $0.029 \%$ & $0.024 \%$ & 0.019 & $99.095 \%$ \\
\hline 521 & *26:01 & *40:02 & *14:05 & $0.029 \%$ & $0.017 \%$ & 0.008 & $99.097 \%$ \\
\hline 521 & *26:01 & *44:03 & ${ }^{\star} 13: 02$ & $0.029 \%$ & $0.003 \%$ & 0.000 & $99.100 \%$ \\
\hline 521 & *26:01 & *51:01 & *11:01 & $0.029 \%$ & $0.009 \%$ & 0.003 & $99.104 \%$ \\
\hline 521 & *26:01 & *55:02 & ${ }^{\star} 08: 03$ & $0.029 \%$ & $0.013 \%$ & 0.005 & $99.110 \%$ \\
\hline 521 & *26:01 & *55:02 & *15:01 & $0.029 \%$ & $0.014 \%$ & 0.006 & $99.114 \%$ \\
\hline 521 & *26:01 & *56:01 & *14:54 & $0.029 \%$ & $0.026 \%$ & 0.029 & $99.119 \%$ \\
\hline 521 & *26:02 & *40:06 & *15:01 & $0.029 \%$ & $0.022 \%$ & 0.012 & $99.123 \%$ \\
\hline 521 & *26:03 & *15:01 & ${ }^{\star} 04: 03$ & $0.029 \%$ & $0.024 \%$ & 0.011 & $99.126 \%$ \\
\hline 521 & *26:03 & *35:01 & ${ }^{\star} 04: 05$ & $0.029 \%$ & $0.004 \%$ & 0.002 & $99.128 \%$ \\
\hline 521 & *31:01 & *15:18 & ${ }^{\star} 04: 01$ & $0.029 \%$ & $0.028 \%$ & 0.031 & $99.134 \%$ \\
\hline 521 & $\star 31: 01$ & *37:01 & *10:01 & $0.029 \%$ & $0.029 \%$ & 0.050 & $99.138 \%$ \\
\hline
\end{tabular}




\begin{tabular}{|c|c|c|c|c|c|c|c|}
\hline 521 & *31:01 & *40:01 & *12:01 & $0.029 \%$ & $0.012 \%$ & 0.003 & $99.140 \%$ \\
\hline 521 & *31:01 & $\star 40: 02$ & $\star^{\star} 04: 03$ & $0.029 \%$ & $0.009 \%$ & 0.003 & $99.143 \%$ \\
\hline 521 & *31:01 & *40:03 & *14:05 & $0.029 \%$ & $0.028 \%$ & 0.083 & $99.149 \%$ \\
\hline 521 & *31:01 & *44:03 & ${ }^{*} 08: 03$ & $0.029 \%$ & $-0.019 \%$ & -0.003 & $99.153 \%$ \\
\hline 521 & *31:01 & *51:01 & ${ }^{*} 04: 06$ & $0.029 \%$ & $0.003 \%$ & 0.001 & $99.156 \%$ \\
\hline 521 & *31:01 & *51:01 & *14:06 & $0.029 \%$ & $0.018 \%$ & 0.014 & $99.159 \%$ \\
\hline 521 & *33:03 & *15:01 & ${ }^{*} 09: 01$ & $0.029 \%$ & $-0.049 \%$ & -0.007 & $99.161 \%$ \\
\hline 521 & *33:03 & *15:01 & *14:06 & $0.029 \%$ & $0.022 \%$ & 0.017 & $99.165 \%$ \\
\hline 521 & *33:03 & *40:03 & *14:05 & $0.029 \%$ & $0.028 \%$ & 0.084 & $99.170 \%$ \\
\hline 521 & *33:03 & *40:06 & ${ }^{*} 09: 01$ & $0.029 \%$ & $-0.017 \%$ & -0.004 & $99.173 \%$ \\
\hline 521 & *33:03 & *44:03 & ${ }^{*} 04: 03$ & $0.029 \%$ & $0.017 \%$ & 0.006 & $99.176 \%$ \\
\hline 521 & *33:03 & *51:01 & *09:01 & $0.029 \%$ & $-0.064 \%$ & -0.009 & $99.180 \%$ \\
\hline 604 & ${ }^{\star} 02: 01$ & *15:01 & ${ }^{*} 04: 03$ & $0.023 \%$ & $-0.002 \%$ & -0.001 & $99.183 \%$ \\
\hline 604 & ${ }^{\star} 02: 01$ & *15:18 & *14:54 & $0.023 \%$ & $0.017 \%$ & 0.012 & $99.186 \%$ \\
\hline 604 & ${ }^{\star} 02: 01$ & *35:01 & ${ }^{\star} 01: 01$ & $0.023 \%$ & $-0.032 \%$ & -0.006 & $99.191 \%$ \\
\hline 604 & ${ }^{\star} 02: 01$ & *35:01 & *14:03 & $0.023 \%$ & $0.008 \%$ & 0.005 & $99.194 \%$ \\
\hline 604 & *02:01 & *35:01 & *14:06 & $0.023 \%$ & $0.011 \%$ & 0.009 & $99.196 \%$ \\
\hline 604 & *02:01 & *38:02 & ${ }^{*} 04: 10$ & $0.023 \%$ & $0.022 \%$ & 0.081 & $99.200 \%$ \\
\hline 604 & *02:01 & *39:01 & ${ }^{*} 04: 05$ & $0.023 \%$ & $-0.031 \%$ & -0.009 & $99.203 \%$ \\
\hline 604 & *02:01 & *39:01 & ${ }^{*} 08: 02$ & $0.023 \%$ & $0.006 \%$ & 0.002 & $99.205 \%$ \\
\hline 604 & *02:01 & *40:01 & ${ }^{*} 04: 06$ & $0.023 \%$ & $0.003 \%$ & 0.001 & $99.208 \%$ \\
\hline 604 & ${ }^{*} 02: 01$ & *40:01 & *15:02 & $0.023 \%$ & $-0.043 \%$ & -0.008 & $99.212 \%$ \\
\hline 604 & *02:01 & *40:02 & *12:01 & $0.023 \%$ & $-0.011 \%$ & -0.003 & $99.214 \%$ \\
\hline 604 & *02:01 & *40:06 & ${ }^{*} 08: 03$ & $0.023 \%$ & $-0.023 \%$ & -0.005 & $99.219 \%$ \\
\hline 604 & *02:01 & *40:06 & *12:02 & $0.023 \%$ & $0.014 \%$ & 0.008 & $99.223 \%$ \\
\hline 604 & ${ }^{\star} 02: 01$ & *44:02 & *13:01 & $0.023 \%$ & $0.023 \%$ & 0.060 & $99.228 \%$ \\
\hline 604 & ${ }^{\star} 02: 01$ & *51:01 & *11:01 & $0.023 \%$ & $-0.007 \%$ & -0.003 & $99.229 \%$ \\
\hline 604 & *02:06 & *15:01 & ${ }^{*} 04: 05$ & $0.023 \%$ & $-0.075 \%$ & -0.010 & $99.230 \%$ \\
\hline 604 & *02:06 & *15:01 & ${ }^{*} 08: 02$ & $0.023 \%$ & $-0.008 \%$ & -0.002 & $99.232 \%$ \\
\hline 604 & *02:06 & *15:01 & ${ }^{*} 12: 01$ & $0.023 \%$ & $-0.003 \%$ & -0.001 & $99.236 \%$ \\
\hline 604 & ${ }^{\star} 02: 06$ & *15:01 & *14:54 & $0.023 \%$ & $-0.002 \%$ & -0.001 & $99.238 \%$ \\
\hline 604 & ${ }^{\star} 02: 06$ & *35:01 & *14:06 & $0.023 \%$ & $0.014 \%$ & 0.011 & $99.240 \%$ \\
\hline 604 & ${ }^{\star} 02: 06$ & *40:02 & ${ }^{\star} 04: 01$ & $0.023 \%$ & $0.016 \%$ & 0.018 & $99.242 \%$ \\
\hline 604 & ${ }^{\star} 02: 06$ & *40:02 & *15:02 & $0.023 \%$ & $-0.057 \%$ & -0.007 & $99.246 \%$ \\
\hline 604 & ${ }^{\star} 02: 06$ & *48:01 & *16:02 & $0.023 \%$ & $0.021 \%$ & 0.021 & $99.249 \%$ \\
\hline 604 & ${ }^{\star} 02: 06$ & $\star 51: 01$ & ${ }^{\star} 04: 03$ & $0.023 \%$ & $-0.001 \%$ & 0.000 & $99.251 \%$ \\
\hline 604 & *02:06 & $\star 51: 01$ & *11:01 & $0.023 \%$ & $-0.001 \%$ & 0.000 & $99.252 \%$ \\
\hline 604 & ${ }^{*} 02: 06$ & *55:02 & *11:01 & $0.023 \%$ & $0.017 \%$ & 0.007 & $99.255 \%$ \\
\hline 604 & ${ }^{*} 02: 06$ & *67:01 & *16:02 & $0.023 \%$ & $0.022 \%$ & 0.023 & $99.258 \%$ \\
\hline 604 & ${ }^{\star} 02: 07$ & *15:01 & *12:01 & $0.023 \%$ & $0.013 \%$ & 0.004 & $99.260 \%$ \\
\hline 604 & ${ }^{\star} 02: 07$ & *40:02 & *08:03 & $0.023 \%$ & $-0.001 \%$ & 0.000 & $99.263 \%$ \\
\hline 604 & ${ }^{\star} 02: 07$ & *46:01 & *14:05 & $0.023 \%$ & $0.020 \%$ & 0.010 & $99.269 \%$ \\
\hline 604 & *11:01 & *15:01 & *11:01 & $0.023 \%$ & $0.004 \%$ & 0.001 & $99.272 \%$ \\
\hline 604 & *11:01 & *15:01 & *14:54 & $0.023 \%$ & $-0.001 \%$ & 0.000 & $99.276 \%$ \\
\hline 604 & *11:01 & *15:01 & *14:06 & $0.023 \%$ & $0.014 \%$ & 0.011 & $99.278 \%$ \\
\hline 604 & *11:01 & *15:01 & *15:02 & $0.023 \%$ & $-0.051 \%$ & -0.007 & $99.282 \%$ \\
\hline 604 & *11:01 & *15:02 & ${ }^{\star} 12: 02$ & $0.023 \%$ & $0.023 \%$ & 0.499 & $99.287 \%$ \\
\hline 604 & *11:01 & *15:18 & ${ }^{\star} 04: 01$ & $0.023 \%$ & $0.022 \%$ & 0.025 & $99.291 \%$ \\
\hline 604 & *11:01 & *35:01 & ${ }^{\star} 08: 03$ & $0.023 \%$ & $-0.040 \%$ & -0.005 & $99.294 \%$ \\
\hline 604 & *11:01 & $\star 40: 02$ & *15:01 & $0.023 \%$ & $-0.033 \%$ & -0.004 & $99.296 \%$ \\
\hline 604 & *11:01 & *51:01 & ${ }^{\star} 04: 01$ & $0.023 \%$ & $0.016 \%$ & 0.018 & $99.299 \%$ \\
\hline 604 & *11:01 & $\star 52: 01$ & ${ }^{\star 04: 05}$ & $0.023 \%$ & $-0.115 \%$ & -0.013 & $99.300 \%$ \\
\hline 604 & *11:01 & $\star 54: 01$ & *14:54 & $0.023 \%$ & $-0.001 \%$ & 0.000 & $99.304 \%$ \\
\hline 604 & *11:01 & *54:01 & *15:01 & $0.023 \%$ & $-0.030 \%$ & -0.004 & $99.306 \%$ \\
\hline
\end{tabular}




\begin{tabular}{|c|c|c|c|c|c|c|c|}
\hline 604 & *11:01 & *55:02 & ${ }^{\star} 01: 01$ & $0.023 \%$ & $0.010 \%$ & 0.004 & $99.311 \%$ \\
\hline 604 & *11:01 & *55:02 & ${ }^{\star} 08: 02$ & $0.023 \%$ & $0.014 \%$ & 0.006 & $99.314 \%$ \\
\hline 604 & *11:01 & *55:02 & *15:01 & $0.023 \%$ & $0.006 \%$ & 0.002 & $99.315 \%$ \\
\hline 604 & $\star 11: 01$ & *67:01 & *14:05 & $0.023 \%$ & $0.021 \%$ & 0.018 & $99.320 \%$ \\
\hline 604 & *11:02 & *27:04 & ${ }^{\star} 12: 02$ & $0.023 \%$ & $0.023 \%$ & 0.138 & $99.326 \%$ \\
\hline 604 & *24:02 & *07:02 & ${ }^{\star} 04: 10$ & $0.023 \%$ & $-0.020 \%$ & -0.010 & $99.328 \%$ \\
\hline 604 & *24:02 & ${ }^{*} 07: 02$ & *11:01 & $0.023 \%$ & $-0.034 \%$ & -0.012 & $99.330 \%$ \\
\hline 604 & *24:02 & *07:02 & *14:03 & $0.023 \%$ & $-0.010 \%$ & -0.006 & $99.332 \%$ \\
\hline 604 & *24:02 & *15:01 & ${ }^{*} 04: 10$ & $0.023 \%$ & $-0.036 \%$ & -0.018 & $99.336 \%$ \\
\hline 604 & *24:02 & *15:01 & *12:01 & $0.023 \%$ & $-0.079 \%$ & -0.022 & $99.339 \%$ \\
\hline 604 & *24:02 & *15:01 & *14:54 & $0.023 \%$ & $-0.074 \%$ & -0.022 & $99.340 \%$ \\
\hline 604 & *24:02 & *15:07 & ${ }^{*} 04: 06$ & $0.023 \%$ & $0.016 \%$ & 0.028 & $99.344 \%$ \\
\hline 604 & *24:02 & *15:07 & *15:01 & $0.023 \%$ & $0.007 \%$ & 0.012 & $99.346 \%$ \\
\hline 604 & *24:02 & *15:18 & ${ }^{*} 04: 06$ & $0.023 \%$ & $0.007 \%$ & 0.005 & $99.349 \%$ \\
\hline 604 & *24:02 & *15:27 & ${ }^{*} 04: 06$ & $0.023 \%$ & $0.022 \%$ & 0.326 & $99.351 \%$ \\
\hline 604 & *24:02 & *15:27 & *09:01 & $0.023 \%$ & $0.020 \%$ & 0.297 & $99.353 \%$ \\
\hline 604 & *24:02 & *35:01 & ${ }^{*} 04: 04$ & $0.023 \%$ & $0.015 \%$ & 0.059 & $99.355 \%$ \\
\hline 604 & $\star 24: 02$ & *35:01 & *14:03 & $0.023 \%$ & $-0.024 \%$ & -0.015 & $99.358 \%$ \\
\hline 604 & $\star 24: 02$ & *35:01 & *14:05 & $0.023 \%$ & $-0.035 \%$ & -0.018 & $99.361 \%$ \\
\hline 604 & $\star 24: 02$ & *35:01 & ${ }^{\star} 15: 02$ & $0.023 \%$ & $-0.289 \%$ & -0.037 & $99.364 \%$ \\
\hline 604 & $\star 24: 02$ & *39:01 & ${ }^{\star} 14: 06$ & $0.023 \%$ & $0.007 \%$ & 0.006 & $99.368 \%$ \\
\hline 604 & $\star 24: 02$ & *40:01 & ${ }^{\star} 04: 01$ & $0.023 \%$ & $0.006 \%$ & 0.007 & $99.370 \%$ \\
\hline 604 & $\star 24: 02$ & *40:01 & ${ }^{\star} 15: 02$ & $0.023 \%$ & $-0.183 \%$ & -0.036 & $99.372 \%$ \\
\hline 604 & $\star 24: 02$ & *40:02 & ${ }^{\star} 14: 03$ & $0.023 \%$ & $-0.023 \%$ & -0.015 & $99.374 \%$ \\
\hline 604 & *24:02 & *40:02 & *14:06 & $0.023 \%$ & $-0.014 \%$ & -0.011 & $99.376 \%$ \\
\hline 604 & *24:02 & *40:03 & *14:05 & $0.023 \%$ & $0.021 \%$ & 0.061 & $99.378 \%$ \\
\hline 604 & *24:02 & *40:06 & ${ }^{*} 08: 03$ & $0.023 \%$ & $-0.118 \%$ & -0.027 & $99.382 \%$ \\
\hline 604 & *24:02 & *51:01 & ${ }^{\star} 01: 01$ & $0.023 \%$ & $-0.172 \%$ & -0.031 & $99.384 \%$ \\
\hline 604 & *24:02 & *51:01 & *12:02 & $0.023 \%$ & $-0.038 \%$ & -0.021 & $99.386 \%$ \\
\hline 604 & *24:02 & $\star 51: 01$ & *16:02 & $0.023 \%$ & $-0.010 \%$ & -0.010 & $99.388 \%$ \\
\hline 604 & *24:02 & $\star 52: 01$ & *13:02 & $0.023 \%$ & $-0.203 \%$ & -0.038 & $99.389 \%$ \\
\hline 604 & *24:02 & *59:01 & ${ }^{\star} 08: 03$ & $0.023 \%$ & $-0.036 \%$ & -0.020 & $99.392 \%$ \\
\hline 604 & *24:02 & *59:01 & ${ }^{*} 12: 01$ & $0.023 \%$ & $-0.002 \%$ & -0.001 & $99.394 \%$ \\
\hline 604 & *24:02 & *59:01 & *15:01 & $0.023 \%$ & $-0.030 \%$ & -0.016 & $99.396 \%$ \\
\hline 604 & *26:01 & *15:18 & ${ }^{*} 04: 01$ & $0.023 \%$ & $0.022 \%$ & 0.025 & $99.398 \%$ \\
\hline 604 & *26:01 & *35:01 & *14:06 & $0.023 \%$ & $0.015 \%$ & 0.012 & $99.400 \%$ \\
\hline 604 & *26:01 & *40:01 & ${ }^{*} 08: 02$ & $0.023 \%$ & $0.006 \%$ & 0.001 & $99.402 \%$ \\
\hline 604 & $\star 26: 01$ & *48:01 & ${ }^{\star} 04: 03$ & $0.023 \%$ & $0.017 \%$ & 0.006 & $99.406 \%$ \\
\hline 604 & $\star 26: 01$ & *51:01 & ${ }^{\star} 04: 06$ & $0.023 \%$ & $0.000 \%$ & 0.000 & $99.408 \%$ \\
\hline 604 & $\star 26: 01$ & *51:01 & ${ }^{\star} 08: 03$ & $0.023 \%$ & $-0.038 \%$ & -0.005 & $99.409 \%$ \\
\hline 604 & $\star 26: 01$ & $\star 55: 02$ & ${ }^{\star} 04: 10$ & $0.023 \%$ & $0.019 \%$ & 0.009 & $99.413 \%$ \\
\hline 604 & *26:01 & *55:02 & ${ }^{\star} 08: 02$ & $0.023 \%$ & $0.015 \%$ & 0.006 & $99.415 \%$ \\
\hline 604 & *26:01 & *56:01 & ${ }^{\star} 04: 05$ & $0.023 \%$ & $0.014 \%$ & 0.015 & $99.417 \%$ \\
\hline 604 & *26:02 & *15:01 & $\star 12: 01$ & $0.023 \%$ & $0.018 \%$ & 0.010 & $99.419 \%$ \\
\hline 604 & *26:02 & *40:02 & ${ }^{\star} 09: 01$ & $0.023 \%$ & $0.002 \%$ & 0.001 & $99.422 \%$ \\
\hline 604 & *26:02 & *44:03 & ${ }^{\star} 13: 02$ & $0.023 \%$ & $0.017 \%$ & 0.009 & $99.423 \%$ \\
\hline 604 & *26:03 & *35:01 & ${ }^{\star} 09: 01$ & $0.023 \%$ & $-0.003 \%$ & -0.001 & $99.426 \%$ \\
\hline 604 & *26:03 & *35:01 & *11:01 & $0.023 \%$ & $0.018 \%$ & 0.008 & $99.428 \%$ \\
\hline 604 & *26:03 & *51:01 & *15:01 & $0.023 \%$ & $0.007 \%$ & 0.003 & $99.429 \%$ \\
\hline 604 & *26:05 & *51:01 & *14:54 & $0.023 \%$ & $0.023 \%$ & 0.305 & $99.432 \%$ \\
\hline 604 & *29:01 & $* 07: 05$ & ${ }^{\star} 08: 03$ & $0.023 \%$ & $0.023 \%$ & 0.667 & $99.436 \%$ \\
\hline 604 & *31:01 & $\star 15: 01$ & ${ }^{*} 04: 03$ & $0.023 \%$ & $0.004 \%$ & 0.001 & $99.437 \%$ \\
\hline 604 & *31:01 & *35:01 & ${ }^{\star} 04: 07$ & $0.023 \%$ & $0.020 \%$ & 0.040 & $99.440 \%$ \\
\hline 604 & *31:01 & *35:01 & ${ }^{*} 08: 03$ & $0.023 \%$ & $-0.039 \%$ & -0.005 & $99.441 \%$ \\
\hline
\end{tabular}




\begin{tabular}{|c|c|c|c|c|c|c|c|}
\hline 604 & $\star 31: 01$ & *35:01 & *14:54 & $0.023 \%$ & $-0.002 \%$ & -0.001 & $99.443 \%$ \\
\hline 604 & *31:01 & *48:01 & *15:01 & $0.023 \%$ & $0.004 \%$ & 0.001 & $99.445 \%$ \\
\hline 604 & *31:01 & *54:01 & ${ }^{\star} 09: 01$ & $0.023 \%$ & $-0.073 \%$ & -0.010 & $99.447 \%$ \\
\hline 604 & *31:01 & *55:02 & ${ }^{\star} 04: 05$ & $0.023 \%$ & $-0.007 \%$ & -0.003 & $99.450 \%$ \\
\hline 604 & *31:01 & *55:02 & ${ }^{\star} 09: 01$ & $0.023 \%$ & $-0.008 \%$ & -0.003 & $99.452 \%$ \\
\hline 604 & *33:03 & *15:01 & ${ }^{\star} 04: 06$ & $0.023 \%$ & $0.006 \%$ & 0.002 & $99.453 \%$ \\
\hline 604 & *33:03 & *40:02 & ${ }^{*} 08: 02$ & $0.023 \%$ & $-0.001 \%$ & 0.000 & $99.455 \%$ \\
\hline 604 & *33:03 & $\star 51: 01$ & *14:03 & $0.023 \%$ & $0.013 \%$ & 0.008 & $99.457 \%$ \\
\hline 604 & *33:03 & $\star 54: 01$ & *08:03 & $0.023 \%$ & $-0.022 \%$ & -0.003 & $99.459 \%$ \\
\hline 604 & *33:03 & $\star 54: 01$ & *15:01 & $0.023 \%$ & $-0.018 \%$ & -0.003 & $99.461 \%$ \\
\hline 710 & *01:01 & $\star 37: 01$ & *04:05 & $0.017 \%$ & $0.017 \%$ & 0.036 & $99.465 \%$ \\
\hline 710 & *01:01 & $\star 37: 01$ & *15:02 & $0.017 \%$ & $0.017 \%$ & 0.036 & $99.469 \%$ \\
\hline 710 & *02:01 & *13:01 & *13:02 & $0.017 \%$ & $0.010 \%$ & 0.008 & $99.472 \%$ \\
\hline 710 & *02:01 & *13:01 & *14:05 & $0.017 \%$ & $0.015 \%$ & 0.012 & $99.474 \%$ \\
\hline 710 & *02:01 & *15:01 & *12:01 & $0.017 \%$ & $-0.016 \%$ & -0.004 & $99.475 \%$ \\
\hline 710 & *02:01 & *15:01 & *14:54 & $0.017 \%$ & $-0.014 \%$ & -0.004 & $99.476 \%$ \\
\hline 710 & ${ }^{\star} 02: 01$ & *15:07 & *04:03 & $0.017 \%$ & $0.015 \%$ & 0.026 & $99.479 \%$ \\
\hline 710 & ${ }^{\star} 02: 01$ & *15:11 & ${ }^{\star} 08: 03$ & $0.017 \%$ & $0.008 \%$ & 0.008 & $99.481 \%$ \\
\hline 710 & ${ }^{\star} 02: 01$ & *15:18 & ${ }^{\star} 04: 03$ & $0.017 \%$ & $0.013 \%$ & 0.009 & $99.483 \%$ \\
\hline 710 & ${ }^{\star} 02: 01$ & *15:18 & *12:01 & $0.017 \%$ & $0.011 \%$ & 0.008 & $99.484 \%$ \\
\hline 710 & ${ }^{\star} 02: 01$ & *15:18 & $\star 15: 01$ & $0.017 \%$ & $0.004 \%$ & 0.003 & $99.486 \%$ \\
\hline 710 & ${ }^{\star} 02: 01$ & *39:01 & $\star 14: 06$ & $0.017 \%$ & $0.012 \%$ & 0.010 & $99.487 \%$ \\
\hline 710 & ${ }^{\star} 02: 01$ & *40:01 & ${ }^{*} 01: 01$ & $0.017 \%$ & $-0.019 \%$ & -0.004 & $99.489 \%$ \\
\hline 710 & ${ }^{\star} 02: 01$ & *40:01 & ${ }^{*} 04: 03$ & $0.017 \%$ & $0.000 \%$ & 0.000 & $99.490 \%$ \\
\hline 710 & ${ }^{\star} 02: 01$ & *40:01 & ${ }^{*} 04: 10$ & $0.017 \%$ & $0.004 \%$ & 0.002 & $99.491 \%$ \\
\hline 710 & ${ }^{\star} 02: 01$ & *40:03 & ${ }^{\star} 08: 02$ & $0.017 \%$ & $0.016 \%$ & 0.046 & $99.494 \%$ \\
\hline 710 & ${ }^{\star} 02: 01$ & *40:03 & *12:01 & $0.017 \%$ & $0.016 \%$ & 0.047 & $99.495 \%$ \\
\hline 710 & ${ }^{\star} 02: 01$ & *44:03 & ${ }^{*} 04: 05$ & $0.017 \%$ & $-0.082 \%$ & -0.013 & $99.496 \%$ \\
\hline 710 & ${ }^{\star} 02: 01$ & *48:01 & *04:05 & $0.017 \%$ & $-0.027 \%$ & -0.010 & $99.497 \%$ \\
\hline 710 & ${ }^{\star} 02: 01$ & *48:01 & *04:06 & $0.017 \%$ & $0.007 \%$ & 0.002 & $99.499 \%$ \\
\hline 710 & ${ }^{*} 02: 01$ & *48:01 & *14:05 & $0.017 \%$ & $0.011 \%$ & 0.005 & $99.501 \%$ \\
\hline 710 & *02:01 & *54:01 & *14:05 & $0.017 \%$ & $0.000 \%$ & 0.000 & $99.503 \%$ \\
\hline 710 & ${ }^{\star} 02: 01$ & *55:02 & ${ }^{\star} 04: 06$ & $0.017 \%$ & $0.008 \%$ & 0.003 & $99.505 \%$ \\
\hline 710 & ${ }^{\star} 02: 01$ & *55:02 & ${ }^{*} 12: 02$ & $0.017 \%$ & $0.012 \%$ & 0.007 & $99.507 \%$ \\
\hline 710 & ${ }^{\star} 02: 01$ & *56:01 & *14:05 & $0.017 \%$ & $0.015 \%$ & 0.017 & $99.509 \%$ \\
\hline 710 & ${ }^{\star} 02: 01$ & ${ }^{*} 67: 01$ & *15:01 & $0.017 \%$ & $0.007 \%$ & 0.006 & $99.510 \%$ \\
\hline 710 & ${ }^{\star} 02: 06$ & *15:01 & ${ }^{\star} 04: 01$ & $0.017 \%$ & $0.011 \%$ & 0.012 & $99.512 \%$ \\
\hline 710 & *02:06 & *15:01 & *14:05 & $0.017 \%$ & $0.003 \%$ & 0.002 & $99.514 \%$ \\
\hline 710 & ${ }^{\star} 02: 06$ & *15:18 & ${ }^{\star} 09: 01$ & $0.017 \%$ & $-0.002 \%$ & -0.001 & $99.515 \%$ \\
\hline 710 & ${ }^{\star} 02: 06$ & *15:18 & *15:01 & $0.017 \%$ & $0.007 \%$ & 0.005 & $99.516 \%$ \\
\hline 710 & ${ }^{\star} 02: 06$ & *27:04 & ${ }^{\star} 04: 05$ & $0.017 \%$ & $0.015 \%$ & 0.074 & $99.518 \%$ \\
\hline 710 & ${ }^{\star} 02: 06$ & *27:05 & ${ }^{\star} 01: 01$ & $0.017 \%$ & $0.017 \%$ & 0.210 & $99.520 \%$ \\
\hline 710 & ${ }^{\star} 02: 06$ & *35:01 & ${ }^{\star} 04: 01$ & $0.017 \%$ & $0.011 \%$ & 0.012 & $99.522 \%$ \\
\hline 710 & ${ }^{\star} 02: 06$ & *35:01 & ${ }^{\star} 04: 06$ & $0.017 \%$ & $-0.007 \%$ & -0.002 & $99.524 \%$ \\
\hline 710 & ${ }^{\star} 02: 06$ & $\star 35: 01$ & ${ }^{*} 04: 10$ & $0.017 \%$ & $0.001 \%$ & 0.001 & $99.525 \%$ \\
\hline 710 & ${ }^{*} 02: 06$ & *39:01 & *04:06 & $0.017 \%$ & $0.007 \%$ & 0.002 & $99.527 \%$ \\
\hline 710 & ${ }^{\star} 02: 06$ & *39:01 & ${ }^{*} 04: 10$ & $0.017 \%$ & $0.011 \%$ & 0.005 & $99.529 \%$ \\
\hline 710 & ${ }^{\star} 02: 06$ & *40:01 & *11:01 & $0.017 \%$ & $0.003 \%$ & 0.001 & $99.530 \%$ \\
\hline 710 & *02:06 & *40:01 & *15:02 & $0.017 \%$ & $-0.036 \%$ & -0.007 & $99.532 \%$ \\
\hline 710 & *02:06 & *40:02 & *08:03 & $0.017 \%$ & $-0.047 \%$ & -0.006 & $99.533 \%$ \\
\hline 710 & ${ }^{\star} 02: 06$ & *40:06 & *04:06 & $0.017 \%$ & $0.004 \%$ & 0.001 & $99.535 \%$ \\
\hline 710 & $\star 02: 06$ & *40:06 & ${ }^{\star} 08: 02$ & $0.017 \%$ & $-0.001 \%$ & 0.000 & $99.537 \%$ \\
\hline 710 & *02:06 & *40:06 & *08:03 & $0.017 \%$ & $-0.019 \%$ & -0.004 & $99.539 \%$ \\
\hline 710 & *02:06 & *46:01 & ${ }^{*} 04: 05$ & $0.017 \%$ & $-0.044 \%$ & -0.009 & $99.540 \%$ \\
\hline
\end{tabular}




\begin{tabular}{|c|c|c|c|c|c|c|c|}
\hline 710 & ${ }^{\star} 02: 06$ & *48:01 & ${ }^{\star} 12: 02$ & $0.017 \%$ & $0.013 \%$ & 0.007 & $99.542 \%$ \\
\hline 710 & ${ }^{\star} 02: 06$ & *51:01 & ${ }^{*} 04: 10$ & $0.017 \%$ & $-0.001 \%$ & 0.000 & $99.543 \%$ \\
\hline 710 & ${ }^{\star} 02: 06$ & *55:02 & *15:02 & $0.017 \%$ & $-0.007 \%$ & -0.003 & $99.545 \%$ \\
\hline 710 & ${ }^{\star} 02: 06$ & *56:01 & ${ }^{\star} 04: 05$ & $0.017 \%$ & $0.006 \%$ & 0.006 & $99.546 \%$ \\
\hline 710 & ${ }^{\star} 02: 06$ & *56:01 & ${ }^{\star} 09: 01$ & $0.017 \%$ & $0.005 \%$ & 0.006 & $99.547 \%$ \\
\hline 710 & ${ }^{\star} 02: 06$ & *59:01 & ${ }^{\star} 08: 03$ & $0.017 \%$ & $0.002 \%$ & 0.001 & $99.548 \%$ \\
\hline 710 & ${ }^{\star} 02: 07$ & *46:01 & ${ }^{*} 04: 06$ & $0.017 \%$ & $0.012 \%$ & 0.004 & $99.551 \%$ \\
\hline 710 & ${ }^{*} 02: 07$ & *46:01 & *11:01 & $0.017 \%$ & $0.013 \%$ & 0.005 & $99.554 \%$ \\
\hline 710 & ${ }^{\star} 02: 07$ & *46:01 & *16:02 & $0.017 \%$ & $0.016 \%$ & 0.016 & $99.556 \%$ \\
\hline 710 & ${ }^{\star} 02: 07$ & $\star 51: 01$ & ${ }^{*} 04: 05$ & $0.017 \%$ & $-0.027 \%$ & -0.008 & $99.558 \%$ \\
\hline 710 & ${ }^{\star} 02: 07$ & *51:01 & ${ }^{*} 09: 01$ & $0.017 \%$ & $-0.029 \%$ & -0.008 & $99.559 \%$ \\
\hline 710 & ${ }^{\star} 02: 07$ & *55:02 & ${ }^{*} 04: 05$ & $0.017 \%$ & $0.006 \%$ & 0.002 & $99.561 \%$ \\
\hline 710 & *03:01 & *07:02 & *15:01 & $0.017 \%$ & $0.016 \%$ & 0.039 & $99.563 \%$ \\
\hline 710 & *03:01 & *13:01 & *12:02 & $0.017 \%$ & $0.017 \%$ & 0.043 & $99.565 \%$ \\
\hline 710 & *11:01 & *13:02 & ${ }^{*} 07: 01$ & $0.017 \%$ & $0.017 \%$ & 0.054 & $99.568 \%$ \\
\hline 710 & *11:01 & *15:01 & *08:02 & $0.017 \%$ & $-0.013 \%$ & -0.003 & $99.569 \%$ \\
\hline 710 & *11:01 & *15:01 & *12:01 & $0.017 \%$ & $-0.008 \%$ & -0.002 & $99.569 \%$ \\
\hline 710 & *11:01 & *15:01 & *14:03 & $0.017 \%$ & $0.006 \%$ & 0.004 & $99.571 \%$ \\
\hline 710 & *11:01 & *15:18 & *15:01 & $0.017 \%$ & $0.007 \%$ & 0.005 & $99.572 \%$ \\
\hline 710 & *11:01 & *35:01 & ${ }^{\star} 04: 03$ & $0.017 \%$ & $-0.003 \%$ & -0.001 & $99.574 \%$ \\
\hline 710 & *11:01 & *35:01 & ${ }^{\star} 04: 10$ & $0.017 \%$ & $0.002 \%$ & 0.001 & $99.575 \%$ \\
\hline 710 & *11:01 & *35:01 & ${ }^{\star} 08: 02$ & $0.017 \%$ & $-0.014 \%$ & -0.003 & $99.576 \%$ \\
\hline 710 & *11:01 & *35:01 & ${ }^{\star} 11: 01$ & $0.017 \%$ & $-0.003 \%$ & -0.001 & $99.577 \%$ \\
\hline 710 & *11:01 & *35:01 & ${ }^{\star} 14: 03$ & $0.017 \%$ & $0.006 \%$ & 0.004 & $99.578 \%$ \\
\hline 710 & *11:01 & *39:01 & *11:01 & $0.017 \%$ & $0.009 \%$ & 0.003 & $99.580 \%$ \\
\hline 710 & *11:01 & *51:01 & ${ }^{*} 04: 10$ & $0.017 \%$ & $0.000 \%$ & 0.000 & $99.582 \%$ \\
\hline 710 & *11:01 & *54:01 & *15:02 & $0.017 \%$ & $-0.055 \%$ & -0.007 & $99.584 \%$ \\
\hline 710 & *11:01 & *55:04 & ${ }^{*} 04: 05$ & $0.017 \%$ & $0.016 \%$ & 0.120 & $99.585 \%$ \\
\hline 710 & *11:01 & $\star 56: 01$ & ${ }^{\star} 09: 01$ & $0.017 \%$ & $0.006 \%$ & 0.006 & $99.586 \%$ \\
\hline 710 & *11:01 & $\star 56: 03$ & ${ }^{*} 09: 01$ & $0.017 \%$ & $0.014 \%$ & 0.065 & $99.587 \%$ \\
\hline 710 & *11:01 & *67:01 & ${ }^{*} 04: 05$ & $0.017 \%$ & $0.003 \%$ & 0.003 & $99.589 \%$ \\
\hline 710 & *11:01 & *67:01 & ${ }^{*} 04: 06$ & $0.017 \%$ & $0.014 \%$ & 0.012 & $99.591 \%$ \\
\hline 710 & *11:01 & *67:01 & ${ }^{*} 09: 01$ & $0.017 \%$ & $0.003 \%$ & 0.002 & $99.592 \%$ \\
\hline 710 & *11:01 & *67:01 & *14:54 & $0.017 \%$ & $0.014 \%$ & 0.012 & $99.594 \%$ \\
\hline 710 & *11:02 & *27:04 & ${ }^{*} 08: 03$ & $0.017 \%$ & $0.017 \%$ & 0.103 & $99.597 \%$ \\
\hline 710 & *11:02 & *51:01 & ${ }^{*} 09: 01$ & $0.017 \%$ & $0.015 \%$ & 0.091 & $99.598 \%$ \\
\hline 710 & *24:02 & ${ }^{* 07: 02}$ & ${ }^{\star} 04: 03$ & $0.017 \%$ & $-0.040 \%$ & -0.015 & $99.599 \%$ \\
\hline 710 & $\star 24: 02$ & *15:01 & ${ }^{\star} 01: 01$ & $0.017 \%$ & $-0.146 \%$ & -0.026 & $99.601 \%$ \\
\hline 710 & $\star 24: 02$ & *15:01 & ${ }^{\star} 04: 01$ & $0.017 \%$ & $-0.008 \%$ & -0.009 & $99.602 \%$ \\
\hline 710 & $\star 24: 02$ & *15:01 & ${ }^{\star} 11: 01$ & $0.017 \%$ & $-0.061 \%$ & -0.022 & $99.603 \%$ \\
\hline 710 & $\star 24: 02$ & *15:07 & ${ }^{\star} 09: 01$ & $0.017 \%$ & $-0.013 \%$ & -0.024 & $99.603 \%$ \\
\hline 710 & *24:02 & *15:11 & ${ }^{\star} 04: 03$ & $0.017 \%$ & $0.008 \%$ & 0.008 & $99.605 \%$ \\
\hline 710 & *24:02 & *15:18 & ${ }^{\star} 08: 02$ & $0.017 \%$ & $-0.005 \%$ & -0.004 & $99.607 \%$ \\
\hline 710 & *24:02 & *15:18 & $\star 13: 02$ & $0.017 \%$ & $-0.012 \%$ & -0.008 & $99.608 \%$ \\
\hline 710 & *24:02 & *15:18 & *14:54 & $0.017 \%$ & $-0.001 \%$ & -0.001 & $99.609 \%$ \\
\hline 710 & *24:02 & *35:01 & ${ }^{\star} 01: 01$ & $0.017 \%$ & $-0.153 \%$ & -0.027 & $99.611 \%$ \\
\hline 710 & *24:02 & *35:01 & ${ }^{\star} 04: 06$ & $0.017 \%$ & $-0.076 \%$ & -0.025 & $99.611 \%$ \\
\hline 710 & *24:02 & *35:01 & *12:02 & $0.017 \%$ & $-0.036 \%$ & -0.020 & $99.612 \%$ \\
\hline 710 & *24:02 & *35:01 & *13:02 & $0.017 \%$ & $-0.145 \%$ & -0.027 & $99.613 \%$ \\
\hline 710 & *24:02 & *39:01 & ${ }^{*} 04: 03$ & $0.017 \%$ & $-0.017 \%$ & -0.006 & $99.614 \%$ \\
\hline 710 & *24:02 & *39:01 & ${ }^{*} 04: 10$ & $0.017 \%$ & $-0.009 \%$ & -0.004 & $99.616 \%$ \\
\hline 710 & *24:02 & *39:01 & *11:01 & $0.017 \%$ & $-0.017 \%$ & -0.006 & $99.617 \%$ \\
\hline 710 & *24:02 & *40:01 & *14:06 & $0.017 \%$ & $-0.007 \%$ & -0.006 & $99.618 \%$ \\
\hline 710 & *24:02 & *40:02 & *15:02 & $0.017 \%$ & $-0.292 \%$ & -0.038 & $99.619 \%$ \\
\hline
\end{tabular}




\begin{tabular}{|c|c|c|c|c|c|c|c|}
\hline 710 & $\star 24: 02$ & $\star 40: 06$ & ${ }^{\star} 08: 02$ & $0.017 \%$ & $-0.053 \%$ & -0.013 & $99.621 \%$ \\
\hline 710 & $\star 24: 02$ & *40:06 & *15:02 & $0.017 \%$ & $-0.158 \%$ & -0.036 & $99.622 \%$ \\
\hline 710 & $\star 24: 02$ & *44:02 & *13:01 & $0.017 \%$ & $0.017 \%$ & 0.043 & $99.624 \%$ \\
\hline 710 & *24:02 & *44:03 & ${ }^{*} 04: 05$ & $0.017 \%$ & $-0.289 \%$ & -0.049 & $99.625 \%$ \\
\hline 710 & *24:02 & *46:01 & *15:01 & $0.017 \%$ & $-0.119 \%$ & -0.025 & $99.626 \%$ \\
\hline 710 & *24:02 & *48:01 & ${ }^{*} 08: 02$ & $0.017 \%$ & $-0.026 \%$ & -0.009 & $99.628 \%$ \\
\hline 710 & *24:02 & *48:01 & *14:05 & $0.017 \%$ & $-0.003 \%$ & -0.001 & $99.629 \%$ \\
\hline 710 & *24:02 & *51:01 & ${ }^{*} 04: 01$ & $0.017 \%$ & $-0.013 \%$ & -0.015 & $99.629 \%$ \\
\hline 710 & *24:02 & *51:01 & ${ }^{\star} 04: 06$ & $0.017 \%$ & $-0.090 \%$ & -0.029 & $99.630 \%$ \\
\hline 710 & *24:02 & *51:01 & *14:06 & $0.017 \%$ & $-0.026 \%$ & -0.021 & $99.630 \%$ \\
\hline 710 & *24:02 & *52:01 & *04:10 & $0.017 \%$ & $-0.069 \%$ & -0.034 & $99.631 \%$ \\
\hline 710 & *24:02 & *54:01 & ${ }^{*} 04: 10$ & $0.017 \%$ & $-0.040 \%$ & -0.020 & $99.632 \%$ \\
\hline 710 & *24:02 & *54:01 & *11:01 & $0.017 \%$ & $-0.059 \%$ & -0.022 & $99.633 \%$ \\
\hline 710 & *24:02 & $\star 55: 02$ & *08:03 & $0.017 \%$ & $-0.059 \%$ & -0.025 & $99.634 \%$ \\
\hline 710 & *24:02 & *56:01 & *08:02 & $0.017 \%$ & $0.003 \%$ & 0.003 & $99.635 \%$ \\
\hline 710 & *24:02 & *59:01 & ${ }^{*} 04: 10$ & $0.017 \%$ & $0.003 \%$ & 0.001 & $99.636 \%$ \\
\hline 710 & *24:02 & *59:01 & *08:02 & $0.017 \%$ & $-0.012 \%$ & -0.006 & $99.637 \%$ \\
\hline 710 & *24:02 & *59:01 & *10:01 & $0.017 \%$ & $0.013 \%$ & 0.024 & $99.638 \%$ \\
\hline 710 & $\star 24: 02$ & $\star 59: 01$ & $\star 15: 02$ & $0.017 \%$ & $-0.056 \%$ & -0.031 & $99.639 \%$ \\
\hline 710 & *24:20 & $\star 54: 01$ & ${ }^{\star} 04: 05$ & $0.017 \%$ & $0.010 \%$ & 0.015 & $99.640 \%$ \\
\hline 710 & *26:01 & ${ }^{\star} 07: 02$ & ${ }^{\star} 09: 01$ & $0.017 \%$ & $-0.045 \%$ & -0.008 & $99.641 \%$ \\
\hline 710 & *26:01 & *13:01 & $\star 12: 02$ & $0.017 \%$ & $0.016 \%$ & 0.013 & $99.643 \%$ \\
\hline 710 & *26:01 & $\star 15: 01$ & ${ }^{\star} 04: 03$ & $0.017 \%$ & $0.001 \%$ & 0.000 & $99.644 \%$ \\
\hline 710 & *26:01 & $\star 15: 01$ & ${ }^{\star} 04: 10$ & $0.017 \%$ & $0.005 \%$ & 0.002 & $99.646 \%$ \\
\hline 710 & *26:01 & *15:01 & *14:02 & $0.017 \%$ & $0.017 \%$ & 0.268 & $99.647 \%$ \\
\hline 710 & *26:01 & *15:01 & *14:06 & $0.017 \%$ & $0.010 \%$ & 0.008 & $99.648 \%$ \\
\hline 710 & *26:01 & *15:01 & $\star 15: 02$ & $0.017 \%$ & $-0.046 \%$ & -0.006 & $99.649 \%$ \\
\hline 710 & *26:01 & *15:11 & ${ }^{\star} 04: 05$ & $0.017 \%$ & $0.007 \%$ & 0.007 & $99.651 \%$ \\
\hline 710 & *26:01 & *15:11 & ${ }^{\star} 09: 01$ & $0.017 \%$ & $0.007 \%$ & 0.007 & $99.651 \%$ \\
\hline 710 & *26:01 & *38:01 & ${ }^{\star} 04: 01$ & $0.017 \%$ & $0.017 \%$ & 0.600 & $99.654 \%$ \\
\hline 710 & *26:01 & *39:01 & ${ }^{\star} 01: 01$ & $0.017 \%$ & $0.002 \%$ & 0.001 & $99.656 \%$ \\
\hline 710 & *26:01 & *39:01 & ${ }^{*} 04: 05$ & $0.017 \%$ & $-0.019 \%$ & -0.005 & $99.657 \%$ \\
\hline 710 & *26:01 & *39:01 & *14:54 & $0.017 \%$ & $0.008 \%$ & 0.002 & $99.659 \%$ \\
\hline 710 & *26:01 & *40:02 & ${ }^{\star} 01: 01$ & $0.017 \%$ & $-0.019 \%$ & -0.003 & $99.661 \%$ \\
\hline 710 & *26:01 & *40:02 & ${ }^{\star} 08: 03$ & $0.017 \%$ & $-0.036 \%$ & -0.005 & $99.662 \%$ \\
\hline 710 & *26:01 & *40:02 & *12:02 & $0.017 \%$ & $0.006 \%$ & 0.003 & $99.663 \%$ \\
\hline 710 & *26:01 & *40:02 & $\star 13: 02$ & $0.017 \%$ & $-0.017 \%$ & -0.003 & $99.665 \%$ \\
\hline 710 & *26:01 & $\star 40: 02$ & $\star 14: 03$ & $0.017 \%$ & $0.008 \%$ & 0.005 & $99.666 \%$ \\
\hline 710 & *26:01 & *40:02 & $\star 15: 02$ & $0.017 \%$ & $-0.048 \%$ & -0.006 & $99.666 \%$ \\
\hline 710 & *26:01 & *40:06 & ${ }^{\star} 04: 06$ & $0.017 \%$ & $0.006 \%$ & 0.002 & $99.668 \%$ \\
\hline 710 & *26:01 & *40:06 & $\star 12: 01$ & $0.017 \%$ & $0.005 \%$ & 0.001 & $99.670 \%$ \\
\hline 710 & *26:01 & *40:06 & *15:01 & $0.017 \%$ & $-0.010 \%$ & -0.002 & $99.671 \%$ \\
\hline 710 & *26:01 & *48:01 & *14:54 & $0.017 \%$ & $0.010 \%$ & 0.003 & $99.673 \%$ \\
\hline 710 & *26:01 & *51:02 & ${ }^{*} 08: 02$ & $0.017 \%$ & $0.017 \%$ & 0.085 & $99.674 \%$ \\
\hline 710 & *26:01 & *52:01 & *14:05 & $0.017 \%$ & $0.000 \%$ & 0.000 & $99.676 \%$ \\
\hline 710 & *26:01 & *54:01 & ${ }^{*} 08: 03$ & $0.017 \%$ & $-0.033 \%$ & -0.004 & $99.677 \%$ \\
\hline 710 & *26:01 & *54:01 & *15:02 & $0.017 \%$ & $-0.044 \%$ & -0.006 & $99.678 \%$ \\
\hline 710 & *26:01 & *55:02 & ${ }^{*} 04: 05$ & $0.017 \%$ & $-0.008 \%$ & -0.003 & $99.679 \%$ \\
\hline 710 & *26:01 & *55:02 & *11:01 & $0.017 \%$ & $0.012 \%$ & 0.005 & $99.680 \%$ \\
\hline 710 & *26:01 & *55:02 & *14:54 & $0.017 \%$ & $0.011 \%$ & 0.004 & $99.681 \%$ \\
\hline 710 & *26:01 & *56:01 & ${ }^{\star} 01: 01$ & $0.017 \%$ & $0.013 \%$ & 0.014 & $99.683 \%$ \\
\hline 710 & *26:01 & *56:01 & *14:05 & $0.017 \%$ & $0.016 \%$ & 0.017 & $99.684 \%$ \\
\hline 710 & *26:02 & *15:01 & *08:03 & $0.017 \%$ & $0.005 \%$ & 0.003 & $99.685 \%$ \\
\hline 710 & *26:02 & *15:01 & *14:03 & $0.017 \%$ & $0.015 \%$ & 0.009 & $99.686 \%$ \\
\hline
\end{tabular}




\begin{tabular}{|c|c|c|c|c|c|c|c|}
\hline 710 & *26:02 & *40:01 & *09:01 & $0.017 \%$ & $0.003 \%$ & 0.002 & $99.688 \%$ \\
\hline 710 & *26:02 & *40:06 & ${ }^{*} 08: 03$ & $0.017 \%$ & $0.010 \%$ & 0.005 & $99.689 \%$ \\
\hline 710 & *26:02 & *48:01 & ${ }^{\star} 09: 01$ & $0.017 \%$ & $0.010 \%$ & 0.005 & $99.690 \%$ \\
\hline 710 & *26:02 & $\star 51: 01$ & ${ }^{\star} 09: 01$ & $0.017 \%$ & $-0.008 \%$ & -0.004 & $99.691 \%$ \\
\hline 710 & *26:02 & $\star 56: 01$ & ${ }^{\star} 09: 01$ & $0.017 \%$ & $0.015 \%$ & 0.016 & $99.692 \%$ \\
\hline 710 & *26:03 & *13:01 & ${ }^{\star} 12: 02$ & $0.017 \%$ & $0.017 \%$ & 0.014 & $99.693 \%$ \\
\hline 710 & $\star 26: 03$ & $\star 15: 01$ & ${ }^{\star} 04: 06$ & $0.017 \%$ & $0.012 \%$ & 0.005 & $99.694 \%$ \\
\hline 710 & *26:03 & $\star 35: 01$ & ${ }^{\star} 04: 10$ & $0.017 \%$ & $0.013 \%$ & 0.006 & $99.695 \%$ \\
\hline 710 & *26:03 & *35:01 & ${ }^{\star} 14: 03$ & $0.017 \%$ & $0.014 \%$ & 0.009 & $99.696 \%$ \\
\hline 710 & *26:03 & *39:01 & ${ }^{\star} 14: 06$ & $0.017 \%$ & $0.016 \%$ & 0.013 & $99.698 \%$ \\
\hline 710 & *26:03 & *40:01 & ${ }^{\star} 04: 05$ & $0.017 \%$ & $0.001 \%$ & 0.000 & $99.699 \%$ \\
\hline 710 & $\star 26: 03$ & *40:06 & ${ }^{\star} 09: 01$ & $0.017 \%$ & $0.003 \%$ & 0.001 & $99.700 \%$ \\
\hline 710 & $\star 26: 03$ & *55:02 & *15:01 & $0.017 \%$ & $0.013 \%$ & 0.006 & $99.701 \%$ \\
\hline 710 & $\star 26: 03$ & *59:01 & ${ }^{\star} 04: 05$ & $0.017 \%$ & $0.011 \%$ & 0.006 & $99.702 \%$ \\
\hline 710 & $\star 26: 05$ & *51:01 & ${ }^{\star} 09: 01$ & $0.017 \%$ & $0.016 \%$ & 0.220 & $99.702 \%$ \\
\hline 710 & *31:01 & *15:01 & *14:54 & $0.017 \%$ & $-0.007 \%$ & -0.002 & $99.703 \%$ \\
\hline 710 & *31:01 & *15:01 & *14:06 & $0.017 \%$ & $0.009 \%$ & 0.007 & $99.704 \%$ \\
\hline 710 & *31:01 & *35:01 & ${ }^{\star} 04: 06$ & $0.017 \%$ & $-0.006 \%$ & -0.002 & $99.704 \%$ \\
\hline 710 & *31:01 & *35:01 & *14:05 & $0.017 \%$ & $0.003 \%$ & 0.002 & $99.705 \%$ \\
\hline 710 & *31:01 & *39:01 & ${ }^{*} 04: 05$ & $0.017 \%$ & $-0.024 \%$ & -0.007 & $99.706 \%$ \\
\hline 710 & *31:01 & *39:01 & ${ }^{\star} 08: 02$ & $0.017 \%$ & $0.004 \%$ & 0.001 & $99.707 \%$ \\
\hline 710 & *31:01 & $\star 39: 02$ & *14:54 & $0.017 \%$ & $0.017 \%$ & 0.060 & $99.709 \%$ \\
\hline 710 & *31:01 & *40:01 & ${ }^{\star} 15: 01$ & $0.017 \%$ & $-0.020 \%$ & -0.004 & $99.710 \%$ \\
\hline 710 & *31:01 & *40:02 & ${ }^{\star} 12: 01$ & $0.017 \%$ & $-0.009 \%$ & -0.002 & $99.711 \%$ \\
\hline 710 & *31:01 & $\star 40: 02$ & ${ }^{\star} 13: 02$ & $0.017 \%$ & $-0.022 \%$ & -0.004 & $99.712 \%$ \\
\hline 710 & *31:01 & $\star 40: 06$ & ${ }^{\star} 08: 03$ & $0.017 \%$ & $-0.017 \%$ & -0.004 & $99.714 \%$ \\
\hline 710 & $\star 31: 01$ & $\star 40: 06$ & ${ }^{\star} 12: 01$ & $0.017 \%$ & $0.003 \%$ & 0.001 & $99.715 \%$ \\
\hline 710 & $\star 31: 01$ & $\star 40: 06$ & ${ }^{\star} 14: 54$ & $0.017 \%$ & $0.003 \%$ & 0.001 & $99.717 \%$ \\
\hline 710 & $\star 31: 01$ & $\star 40: 06$ & *14:05 & $0.017 \%$ & $0.009 \%$ & 0.005 & $99.719 \%$ \\
\hline 710 & $\star 31: 01$ & $\star 46: 01$ & ${ }^{\star} 09: 01$ & $0.017 \%$ & $-0.045 \%$ & -0.009 & $99.719 \%$ \\
\hline 710 & *31:01 & $\star 48: 01$ & ${ }^{\star} 08: 02$ & $0.017 \%$ & $0.007 \%$ & 0.002 & $99.721 \%$ \\
\hline 710 & *31:01 & $\star 51: 01$ & ${ }^{\star} 01: 01$ & $0.017 \%$ & $-0.031 \%$ & -0.005 & $99.722 \%$ \\
\hline 710 & *31:01 & $\star 51: 01$ & ${ }^{\star} 04: 10$ & $0.017 \%$ & $0.000 \%$ & 0.000 & $99.723 \%$ \\
\hline 710 & *31:01 & $\star 51: 01$ & $\star 15: 02$ & $0.017 \%$ & $-0.071 \%$ & -0.008 & $99.723 \%$ \\
\hline 710 & *31:01 & $\star 54: 01$ & ${ }^{\star} 08: 03$ & $0.017 \%$ & $-0.040 \%$ & -0.005 & $99.724 \%$ \\
\hline 710 & *31:01 & $\star 54: 01$ & *13:01 & $0.017 \%$ & $0.013 \%$ & 0.023 & $99.726 \%$ \\
\hline 710 & *31:01 & $\star 54: 01$ & *15:01 & $0.017 \%$ & $-0.035 \%$ & -0.005 & $99.727 \%$ \\
\hline 710 & *31:01 & *55:02 & *08:03 & $0.017 \%$ & $-0.002 \%$ & -0.001 & $99.728 \%$ \\
\hline 710 & *31:01 & *55:02 & *12:01 & $0.017 \%$ & $0.009 \%$ & 0.004 & $99.729 \%$ \\
\hline 710 & *31:01 & *55:02 & *14:54 & $0.017 \%$ & $0.010 \%$ & 0.004 & $99.730 \%$ \\
\hline 710 & *33:03 & *35:01 & $* 04: 10$ & $0.017 \%$ & $0.005 \%$ & 0.003 & $99.731 \%$ \\
\hline 710 & $\star 33: 03$ & $\star 35: 01$ & ${ }^{\star} 09: 01$ & $0.017 \%$ & $-0.064 \%$ & -0.009 & $99.732 \%$ \\
\hline 710 & *33:03 & $\star 44: 03$ & ${ }^{\star} 01: 01$ & $0.017 \%$ & $-0.008 \%$ & -0.001 & $99.734 \%$ \\
\hline 710 & $\star 33: 03$ & $\star 51: 01$ & ${ }^{*} 04: 05$ & $0.017 \%$ & $-0.071 \%$ & -0.010 & $99.735 \%$ \\
\hline 710 & $\star 33: 03$ & $\star 51: 01$ & *11:01 & $0.017 \%$ & $-0.001 \%$ & 0.000 & $99.736 \%$ \\
\hline 710 & *33:03 & $\star 58: 01$ & ${ }^{\star} 04: 06$ & $0.017 \%$ & $0.016 \%$ & 0.026 & $99.738 \%$ \\
\hline 908 & *01:01 & *13:01 & ${ }^{\star} 12: 02$ & $0.012 \%$ & $0.011 \%$ & 0.024 & $99.739 \%$ \\
\hline 908 & *01:01 & $\star 37: 01$ & *14:54 & $0.012 \%$ & $0.011 \%$ & 0.024 & $99.741 \%$ \\
\hline 908 & $\star 01: 01$ & $\star 51: 01$ & *16:02 & $0.012 \%$ & $0.011 \%$ & 0.024 & $99.742 \%$ \\
\hline 908 & *01:01 & *67:01 & *16:02 & $0.012 \%$ & $0.012 \%$ & 0.025 & $99.743 \%$ \\
\hline 908 & *02:01 & *13:01 & *12:01 & $0.012 \%$ & $0.006 \%$ & 0.005 & $99.743 \%$ \\
\hline 908 & *02:01 & *13:01 & *14:54 & $0.012 \%$ & $0.007 \%$ & 0.005 & $99.744 \%$ \\
\hline 908 & $\star 02: 01$ & $\star 15: 01$ & ${ }^{\star} 04: 10$ & $0.012 \%$ & $-0.008 \%$ & -0.004 & $99.745 \%$ \\
\hline 908 & $\star 02: 01$ & $\star 15: 11$ & $\star 04: 10$ & $0.012 \%$ & $0.009 \%$ & 0.009 & $99.746 \%$ \\
\hline
\end{tabular}




\begin{tabular}{|c|c|c|c|c|c|c|c|}
\hline 908 & ${ }^{*} 02: 01$ & *15:11 & ${ }^{\star} 08: 02$ & $0.012 \%$ & $0.007 \%$ & 0.007 & $99.746 \%$ \\
\hline 908 & ${ }^{\star} 02: 01$ & $\star 15: 11$ & $\star 12: 01$ & $0.012 \%$ & $0.007 \%$ & 0.008 & $99.747 \%$ \\
\hline 908 & ${ }^{\star} 02: 01$ & *15:11 & *14:05 & $0.012 \%$ & $0.009 \%$ & 0.010 & $99.748 \%$ \\
\hline 908 & ${ }^{\star} 02: 01$ & *15:18 & *09:01 & $0.012 \%$ & $-0.013 \%$ & -0.009 & $99.748 \%$ \\
\hline 908 & ${ }^{\star} 02: 01$ & *35:01 & *04:06 & $0.012 \%$ & $-0.019 \%$ & -0.006 & $99.748 \%$ \\
\hline 908 & *02:01 & *38:02 & *09:01 & $0.012 \%$ & $0.007 \%$ & 0.025 & $99.749 \%$ \\
\hline 908 & ${ }^{\star} 02: 01$ & *39:01 & ${ }^{*} 01: 01$ & $0.012 \%$ & $-0.012 \%$ & -0.003 & $99.750 \%$ \\
\hline 908 & ${ }^{\star} 02: 01$ & *39:02 & *14:06 & $0.012 \%$ & $0.011 \%$ & 0.040 & $99.751 \%$ \\
\hline 908 & ${ }^{*} 02: 01$ & *39:04 & *04:05 & $0.012 \%$ & $0.008 \%$ & 0.041 & $99.752 \%$ \\
\hline 908 & ${ }^{*} 02: 01$ & *39:04 & *08:03 & $0.012 \%$ & $0.010 \%$ & 0.046 & $99.752 \%$ \\
\hline 908 & *02:01 & *40:01 & *08:09 & $0.012 \%$ & $0.011 \%$ & 0.246 & $99.753 \%$ \\
\hline 908 & *02:01 & *40:02 & ${ }^{*} 04: 06$ & $0.012 \%$ & $-0.018 \%$ & -0.006 & $99.753 \%$ \\
\hline 908 & ${ }^{\star} 02: 01$ & *40:02 & *11:06 & $0.012 \%$ & $0.011 \%$ & 0.494 & $99.754 \%$ \\
\hline 908 & ${ }^{\star} 02: 01$ & *40:02 & $\star 12: 02$ & $0.012 \%$ & $-0.005 \%$ & -0.003 & $99.754 \%$ \\
\hline 908 & ${ }^{\star} 02: 01$ & *40:02 & $\star 13: 02$ & $0.012 \%$ & $-0.040 \%$ & -0.007 & $99.755 \%$ \\
\hline 908 & ${ }^{\star} 02: 01$ & $* 40: 02$ & *14:03 & $0.012 \%$ & $-0.003 \%$ & -0.002 & $99.755 \%$ \\
\hline 908 & *02:01 & *40:03 & *04:05 & $0.012 \%$ & $0.006 \%$ & 0.018 & $99.756 \%$ \\
\hline 908 & *02:01 & *40:06 & *14:54 & $0.012 \%$ & $-0.007 \%$ & -0.002 & $99.757 \%$ \\
\hline 908 & *02:01 & *40:06 & *16:02 & $0.012 \%$ & $0.006 \%$ & 0.007 & $99.758 \%$ \\
\hline 908 & *02:01 & *44:03 & *09:01 & $0.012 \%$ & $-0.093 \%$ & -0.015 & $99.758 \%$ \\
\hline 908 & *02:01 & *46:01 & *01:01 & $0.012 \%$ & $-0.021 \%$ & -0.004 & $99.760 \%$ \\
\hline 908 & ${ }^{*} 02: 01$ & *46:01 & *14:03 & $0.012 \%$ & $0.003 \%$ & 0.002 & $99.761 \%$ \\
\hline 908 & *02:01 & *46:01 & *15:01 & $0.012 \%$ & $-0.032 \%$ & -0.007 & $99.761 \%$ \\
\hline 908 & *02:01 & *46:01 & *15:02 & $0.012 \%$ & $-0.049 \%$ & -0.010 & $99.762 \%$ \\
\hline 908 & *02:01 & *48:01 & *04:07 & $0.012 \%$ & $0.010 \%$ & 0.020 & $99.763 \%$ \\
\hline 908 & ${ }^{\star} 02: 01$ & *48:01 & *16:02 & $0.012 \%$ & $0.008 \%$ & 0.009 & $99.764 \%$ \\
\hline 908 & ${ }^{\star} 02: 01$ & *51:01 & *04:10 & $0.012 \%$ & $-0.011 \%$ & -0.005 & $99.764 \%$ \\
\hline 908 & ${ }^{\star} 02: 01$ & *51:01 & *16:02 & $0.012 \%$ & $0.001 \%$ & 0.001 & $99.765 \%$ \\
\hline 908 & *02:01 & *51:02 & *09:01 & $0.012 \%$ & $0.008 \%$ & 0.043 & $99.765 \%$ \\
\hline 908 & ${ }^{\star} 02: 01$ & *52:01 & *04:05 & $0.012 \%$ & $-0.168 \%$ & -0.015 & $99.765 \%$ \\
\hline 908 & ${ }^{\star} 02: 01$ & *52:01 & *08:03 & $0.012 \%$ & $-0.102 \%$ & -0.012 & $99.766 \%$ \\
\hline 908 & ${ }^{\star} 02: 01$ & *52:01 & *11:01 & $0.012 \%$ & $-0.025 \%$ & -0.009 & $99.766 \%$ \\
\hline 908 & ${ }^{\star} 02: 01$ & *54:01 & *14:54 & $0.012 \%$ & $-0.019 \%$ & -0.006 & $99.767 \%$ \\
\hline 908 & ${ }^{\star} 02: 01$ & *55:02 & ${ }^{\star} 04: 10$ & $0.012 \%$ & $0.006 \%$ & 0.003 & $99.768 \%$ \\
\hline 908 & ${ }^{\star} 02: 01$ & *55:02 & *08:03 & $0.012 \%$ & $-0.013 \%$ & -0.005 & $99.768 \%$ \\
\hline 908 & ${ }^{\star} 02: 01$ & *55:02 & *14:05 & $0.012 \%$ & $0.006 \%$ & 0.003 & $99.769 \%$ \\
\hline 908 & ${ }^{\star} 02: 01$ & *55:04 & *11:01 & $0.012 \%$ & $0.011 \%$ & 0.084 & $99.769 \%$ \\
\hline 908 & ${ }^{*} 02: 01$ & *67:01 & *04:05 & $0.012 \%$ & $-0.007 \%$ & -0.006 & $99.770 \%$ \\
\hline 908 & ${ }^{*} 02: 03$ & *38:02 & *15:01 & $0.012 \%$ & $0.012 \%$ & 0.285 & $99.771 \%$ \\
\hline 908 & ${ }^{\star} 02: 06$ & *15:01 & ${ }^{\star} 01: 01$ & $0.012 \%$ & $-0.030 \%$ & -0.005 & $99.772 \%$ \\
\hline 908 & ${ }^{\star} 02: 06$ & *15:01 & *11:01 & $0.012 \%$ & $-0.009 \%$ & -0.003 & $99.772 \%$ \\
\hline 908 & ${ }^{\star} 02: 06$ & *15:07 & *04:03 & $0.012 \%$ & $0.010 \%$ & 0.017 & $99.773 \%$ \\
\hline 908 & ${ }^{\star} 02: 06$ & *15:11 & *08:03 & $0.012 \%$ & $0.004 \%$ & 0.004 & $99.774 \%$ \\
\hline 908 & ${ }^{\star} 02: 06$ & *15:11 & *12:02 & $0.012 \%$ & $0.010 \%$ & 0.010 & $99.775 \%$ \\
\hline 908 & ${ }^{\star} 02: 06$ & *15:11 & *14:05 & $0.012 \%$ & $0.010 \%$ & 0.010 & $99.776 \%$ \\
\hline 908 & ${ }^{\star} 02: 06$ & *15:18 & ${ }^{\star} 04: 03$ & $0.012 \%$ & $0.008 \%$ & 0.005 & $99.776 \%$ \\
\hline 908 & ${ }^{\star} 02: 06$ & *35:01 & ${ }^{\star} 04: 07$ & $0.012 \%$ & $0.008 \%$ & 0.016 & $99.777 \%$ \\
\hline 908 & $\star 02: 06$ & *35:01 & *13:02 & $0.012 \%$ & $-0.030 \%$ & -0.005 & $99.778 \%$ \\
\hline 908 & $\star 02: 06$ & *35:01 & *15:02 & $0.012 \%$ & $-0.069 \%$ & -0.009 & $99.778 \%$ \\
\hline 908 & $\star 02: 06$ & *39:01 & *11:01 & $0.012 \%$ & $0.003 \%$ & 0.001 & $99.779 \%$ \\
\hline 908 & $\star 02: 06$ & *39:01 & *14:03 & $0.012 \%$ & $0.006 \%$ & 0.004 & $99.779 \%$ \\
\hline 908 & $\star 02: 06$ & *39:01 & *14:05 & $0.012 \%$ & $0.005 \%$ & 0.003 & $99.780 \%$ \\
\hline 908 & *02:06 & *39:04 & ${ }^{\star} 04: 03$ & $0.012 \%$ & $0.011 \%$ & 0.053 & $99.781 \%$ \\
\hline 908 & ${ }^{*} 02: 06$ & *40:01 & ${ }^{\star} 04: 03$ & $0.012 \%$ & $-0.002 \%$ & -0.001 & $99.781 \%$ \\
\hline
\end{tabular}




\begin{tabular}{|c|c|c|c|c|c|c|c|}
\hline 908 & ${ }^{\star} 02: 06$ & $\star 40: 01$ & ${ }^{\star} 12: 02$ & $0.012 \%$ & $0.003 \%$ & 0.001 & $99.782 \%$ \\
\hline 908 & ${ }^{\star} 02: 06$ & $\star 40: 01$ & *14:05 & $0.012 \%$ & $0.002 \%$ & 0.001 & $99.782 \%$ \\
\hline 908 & ${ }^{\star} 02: 06$ & $\star 40: 02$ & ${ }^{\star} 03: 01$ & $0.012 \%$ & $0.011 \%$ & 0.088 & $99.783 \%$ \\
\hline 908 & ${ }^{\star} 02: 06$ & *40:06 & ${ }^{\star} 04: 10$ & $0.012 \%$ & $0.003 \%$ & 0.001 & $99.783 \%$ \\
\hline 908 & ${ }^{\star} 02: 06$ & *44:02 & *13:01 & $0.012 \%$ & $0.011 \%$ & 0.030 & $99.784 \%$ \\
\hline 908 & ${ }^{\star} 02: 06$ & *46:01 & ${ }^{\star} 14: 06$ & $0.012 \%$ & $0.006 \%$ & 0.005 & $99.785 \%$ \\
\hline 908 & ${ }^{\star} 02: 06$ & *48:01 & ${ }^{*} 01: 01$ & $0.012 \%$ & $-0.004 \%$ & -0.001 & $99.786 \%$ \\
\hline 908 & ${ }^{*} 02: 06$ & *48:01 & ${ }^{*} 04: 10$ & $0.012 \%$ & $0.006 \%$ & 0.003 & $99.787 \%$ \\
\hline 908 & ${ }^{*} 02: 06$ & *48:01 & ${ }^{*} 08: 02$ & $0.012 \%$ & $0.000 \%$ & 0.000 & $99.787 \%$ \\
\hline 908 & ${ }^{*} 02: 06$ & *48:01 & *14:06 & $0.012 \%$ & $0.008 \%$ & 0.006 & $99.788 \%$ \\
\hline 908 & *02:06 & *48:01 & *15:02 & $0.012 \%$ & $-0.016 \%$ & -0.006 & $99.789 \%$ \\
\hline 908 & *02:06 & *51:01 & *14:12 & $0.012 \%$ & $0.011 \%$ & 0.395 & $99.789 \%$ \\
\hline 908 & *02:06 & *51:01 & *15:02 & $0.012 \%$ & $-0.081 \%$ & -0.009 & $99.789 \%$ \\
\hline 908 & *02:06 & *54:01 & ${ }^{*} 08: 02$ & $0.012 \%$ & $-0.019 \%$ & -0.004 & $99.790 \%$ \\
\hline 908 & *02:06 & $\star 54: 01$ & *12:01 & $0.012 \%$ & $-0.014 \%$ & -0.004 & $99.790 \%$ \\
\hline 908 & *02:06 & $\star 55: 02$ & ${ }^{*} 04: 03$ & $0.012 \%$ & $0.005 \%$ & 0.002 & $99.791 \%$ \\
\hline 908 & *02:06 & $\star 55: 02$ & ${ }^{*} 04: 06$ & $0.012 \%$ & $0.004 \%$ & 0.002 & $99.792 \%$ \\
\hline 908 & ${ }^{\star} 02: 06$ & $\star 55: 02$ & ${ }^{\star} 12: 01$ & $0.012 \%$ & $0.003 \%$ & 0.001 & $99.792 \%$ \\
\hline 908 & ${ }^{\star} 02: 06$ & $\star 56: 01$ & *14:54 & $0.012 \%$ & $0.009 \%$ & 0.009 & $99.792 \%$ \\
\hline 908 & ${ }^{\star} 02: 06$ & $\star 56: 01$ & ${ }^{\star} 15: 01$ & $0.012 \%$ & $0.005 \%$ & 0.005 & $99.793 \%$ \\
\hline 908 & ${ }^{\star} 02: 06$ & *67:01 & ${ }^{\star} 15: 01$ & $0.012 \%$ & $0.003 \%$ & 0.003 & $99.794 \%$ \\
\hline 908 & ${ }^{\star} 02: 07$ & *13:01 & ${ }^{\star} 12: 02$ & $0.012 \%$ & $0.011 \%$ & 0.009 & $99.794 \%$ \\
\hline 908 & ${ }^{\star} 02: 07$ & *15:01 & ${ }^{\star} 08: 03$ & $0.012 \%$ & $-0.012 \%$ & -0.003 & $99.795 \%$ \\
\hline 908 & ${ }^{\star} 02: 07$ & *15:18 & ${ }^{\star} 04: 01$ & $0.012 \%$ & $0.011 \%$ & 0.012 & $99.795 \%$ \\
\hline 908 & ${ }^{\star} 02: 07$ & *35:01 & ${ }^{*} 04: 05$ & $0.012 \%$ & $-0.027 \%$ & -0.008 & $99.796 \%$ \\
\hline 908 & ${ }^{\star} 02: 07$ & *35:01 & *15:01 & $0.012 \%$ & $-0.010 \%$ & -0.003 & $99.797 \%$ \\
\hline 908 & ${ }^{\star} 02: 07$ & *39:01 & ${ }^{*} 04: 05$ & $0.012 \%$ & $-0.005 \%$ & -0.001 & $99.797 \%$ \\
\hline 908 & ${ }^{*} 02: 07$ & *40:01 & ${ }^{\star} 08: 03$ & $0.012 \%$ & $-0.005 \%$ & -0.001 & $99.798 \%$ \\
\hline 908 & ${ }^{\star} 02: 07$ & *40:06 & ${ }^{*} 09: 01$ & $0.012 \%$ & $-0.011 \%$ & -0.003 & $99.798 \%$ \\
\hline 908 & ${ }^{\star} 02: 07$ & *46:01 & ${ }^{\star} 01: 01$ & $0.012 \%$ & $0.002 \%$ & 0.000 & $99.799 \%$ \\
\hline 908 & *02:07 & *46:01 & ${ }^{*} 04: 10$ & $0.012 \%$ & $0.008 \%$ & 0.004 & $99.800 \%$ \\
\hline 908 & *02:07 & *46:01 & *12:02 & $0.012 \%$ & $0.009 \%$ & 0.005 & $99.801 \%$ \\
\hline 908 & ${ }^{\star} 02: 07$ & *46:01 & *13:02 & $0.012 \%$ & $0.002 \%$ & 0.001 & $99.802 \%$ \\
\hline 908 & ${ }^{\star} 02: 07$ & *46:01 & ${ }^{*} 14: 06$ & $0.012 \%$ & $0.009 \%$ & 0.007 & $99.803 \%$ \\
\hline 908 & ${ }^{\star} 02: 07$ & *48:01 & ${ }^{*} 08: 03$ & $0.012 \%$ & $0.003 \%$ & 0.001 & $99.804 \%$ \\
\hline 908 & ${ }^{\star} 02: 07$ & *48:01 & *14:54 & $0.012 \%$ & $0.008 \%$ & 0.003 & $99.805 \%$ \\
\hline 908 & $\star 02: 07$ & $\star 48: 01$ & *15:01 & $0.012 \%$ & $0.004 \%$ & 0.001 & $99.805 \%$ \\
\hline 908 & ${ }^{\star} 02: 07$ & $\star 51: 01$ & $\star 14: 03$ & $0.012 \%$ & $0.006 \%$ & 0.004 & $99.805 \%$ \\
\hline 908 & ${ }^{\star} 02: 07$ & $\star 51: 01$ & *15:01 & $0.012 \%$ & $-0.014 \%$ & -0.004 & $99.806 \%$ \\
\hline 908 & ${ }^{\star} 02: 07$ & $\star 54: 01$ & ${ }^{\star} 04: 05$ & $0.012 \%$ & $-0.024 \%$ & -0.007 & $99.806 \%$ \\
\hline 908 & ${ }^{\star} 02: 10$ & $\star 15: 07$ & ${ }^{\star} 04: 05$ & $0.012 \%$ & $0.011 \%$ & 0.027 & $99.807 \%$ \\
\hline 908 & ${ }^{\star} 02: 10$ & *40:06 & ${ }^{\star} 08: 03$ & $0.012 \%$ & $0.010 \%$ & 0.024 & $99.808 \%$ \\
\hline 908 & ${ }^{\star} 02: 10$ & *40:06 & $\star 15: 02$ & $0.012 \%$ & $0.010 \%$ & 0.024 & $99.809 \%$ \\
\hline 908 & ${ }^{*} 03: 01$ & *07:02 & ${ }^{\star} 01: 01$ & $0.012 \%$ & $0.010 \%$ & 0.025 & $99.809 \%$ \\
\hline 908 & ${ }^{\star} 03: 01$ & *15:01 & ${ }^{\star} 04: 06$ & $0.012 \%$ & $0.011 \%$ & 0.026 & $99.809 \%$ \\
\hline 908 & *03:01 & *44:02 & ${ }^{\star} 08: 02$ & $0.012 \%$ & $0.012 \%$ & 0.030 & $99.811 \%$ \\
\hline 908 & *11:01 & *07:02 & ${ }^{\star} 15: 01$ & $0.012 \%$ & $-0.028 \%$ & -0.005 & $99.811 \%$ \\
\hline 908 & *11:01 & *13:01 & ${ }^{*} 04: 05$ & $0.012 \%$ & $-0.003 \%$ & -0.003 & $99.812 \%$ \\
\hline 908 & *11:01 & *15:01 & ${ }^{*} 04: 01$ & $0.012 \%$ & $0.005 \%$ & 0.006 & $99.812 \%$ \\
\hline 908 & *11:01 & *15:07 & ${ }^{*} 04: 03$ & $0.012 \%$ & $0.010 \%$ & 0.017 & $99.813 \%$ \\
\hline 908 & *11:01 & *15:07 & ${ }^{*} 09: 01$ & $0.012 \%$ & $0.004 \%$ & 0.007 & $99.814 \%$ \\
\hline 908 & *11:01 & *15:18 & ${ }^{*} 04: 03$ & $0.012 \%$ & $0.008 \%$ & 0.005 & $99.815 \%$ \\
\hline 908 & *11:01 & *27:04 & ${ }^{\star} 04: 05$ & $0.012 \%$ & $0.009 \%$ & 0.046 & $99.815 \%$ \\
\hline 908 & *11:01 & *35:01 & *12:02 & $0.012 \%$ & $-0.002 \%$ & -0.001 & $99.816 \%$ \\
\hline
\end{tabular}




\begin{tabular}{|c|c|c|c|c|c|c|c|}
\hline 908 & *11:01 & *35:01 & *14:54 & $0.012 \%$ & $-0.014 \%$ & -0.004 & $99.816 \%$ \\
\hline 908 & *11:01 & *38:02 & *08:03 & $0.012 \%$ & $0.009 \%$ & 0.034 & $99.817 \%$ \\
\hline 908 & *11:01 & *39:01 & *09:01 & $0.012 \%$ & $-0.033 \%$ & -0.010 & $99.818 \%$ \\
\hline 908 & *11:01 & *40:01 & *12:01 & $0.012 \%$ & $-0.006 \%$ & -0.002 & $99.818 \%$ \\
\hline 908 & *11:01 & *40:01 & *14:05 & $0.012 \%$ & $0.002 \%$ & 0.001 & $99.819 \%$ \\
\hline 908 & *11:01 & *40:01 & *15:02 & $0.012 \%$ & $-0.040 \%$ & -0.007 & $99.819 \%$ \\
\hline 908 & *11:01 & *40:02 & ${ }^{\star} 04: 10$ & $0.012 \%$ & $-0.004 \%$ & -0.002 & $99.820 \%$ \\
\hline 908 & *11:01 & *40:06 & $\star 04: 05$ & $0.012 \%$ & $-0.044 \%$ & -0.010 & $99.821 \%$ \\
\hline 908 & *11:01 & *40:06 & *12:01 & $0.012 \%$ & $-0.003 \%$ & -0.001 & $99.821 \%$ \\
\hline 908 & *11:01 & *46:01 & *15:01 & $0.012 \%$ & $-0.022 \%$ & -0.005 & $99.822 \%$ \\
\hline 908 & *11:01 & *48:01 & ${ }^{\star} 04: 03$ & $0.012 \%$ & $0.005 \%$ & 0.002 & $99.822 \%$ \\
\hline 908 & *11:01 & *48:01 & ${ }^{*} 08: 03$ & $0.012 \%$ & $-0.010 \%$ & -0.004 & $99.823 \%$ \\
\hline 908 & *11:01 & *48:01 & *12:01 & $0.012 \%$ & $0.002 \%$ & 0.001 & $99.824 \%$ \\
\hline 908 & *11:01 & *51:01 & ${ }^{\star} 04: 07$ & $0.012 \%$ & $0.007 \%$ & 0.015 & $99.824 \%$ \\
\hline 908 & *11:01 & *51:01 & ${ }^{\star} 08: 03$ & $0.012 \%$ & $-0.060 \%$ & -0.007 & $99.825 \%$ \\
\hline 908 & *11:01 & *51:01 & *14:54 & $0.012 \%$ & $-0.018 \%$ & -0.005 & $99.825 \%$ \\
\hline 908 & *11:01 & *51:01 & *14:05 & $0.012 \%$ & $-0.005 \%$ & -0.003 & $99.826 \%$ \\
\hline 908 & *11:01 & *51:01 & *15:02 & $0.012 \%$ & $-0.078 \%$ & -0.009 & $99.826 \%$ \\
\hline 908 & *11:01 & *51:01 & *16:02 & $0.012 \%$ & $0.003 \%$ & 0.004 & $99.827 \%$ \\
\hline 908 & *11:01 & *51:02 & *08:02 & $0.012 \%$ & $0.011 \%$ & 0.055 & $99.827 \%$ \\
\hline 908 & *11:01 & *52:01 & *08:03 & $0.012 \%$ & $-0.076 \%$ & -0.009 & $99.828 \%$ \\
\hline 908 & *11:01 & *54:01 & *04:03 & $0.012 \%$ & $-0.007 \%$ & -0.003 & $99.829 \%$ \\
\hline 908 & *11:01 & *54:01 & ${ }^{\star} 04: 06$ & $0.012 \%$ & $-0.010 \%$ & -0.003 & $99.829 \%$ \\
\hline 908 & *11:01 & *54:01 & ${ }^{\star} 04: 10$ & $0.012 \%$ & $-0.003 \%$ & -0.001 & $99.830 \%$ \\
\hline 908 & *11:01 & *54:01 & *11:01 & $0.012 \%$ & $-0.007 \%$ & -0.003 & $99.830 \%$ \\
\hline 908 & *11:01 & *54:01 & *12:01 & $0.012 \%$ & $-0.013 \%$ & -0.004 & $99.831 \%$ \\
\hline 908 & *11:01 & *54:01 & *13:01 & $0.012 \%$ & $0.008 \%$ & 0.013 & $99.831 \%$ \\
\hline 908 & *11:01 & $\star 54: 01$ & *13:02 & $0.012 \%$ & $-0.026 \%$ & -0.005 & $99.832 \%$ \\
\hline 908 & *11:01 & *55:02 & ${ }^{\star} 08: 03$ & $0.012 \%$ & $-0.008 \%$ & -0.003 & $99.832 \%$ \\
\hline 908 & *11:01 & *55:02 & *11:01 & $0.012 \%$ & $0.005 \%$ & 0.002 & $99.832 \%$ \\
\hline 908 & *11:01 & *55:02 & *12:02 & $0.012 \%$ & $0.008 \%$ & 0.004 & $99.833 \%$ \\
\hline 908 & *11:01 & *56:01 & ${ }^{\star} 04: 05$ & $0.012 \%$ & $0.000 \%$ & 0.000 & $99.833 \%$ \\
\hline 908 & *11:01 & *56:03 & *15:01 & $0.012 \%$ & $0.010 \%$ & 0.045 & $99.834 \%$ \\
\hline 908 & *11:01 & *67:01 & *04:03 & $0.012 \%$ & $0.009 \%$ & 0.008 & $99.834 \%$ \\
\hline 908 & *11:02 & *15:07 & ${ }^{*} 04: 03$ & $0.012 \%$ & $0.012 \%$ & 0.069 & $99.835 \%$ \\
\hline 908 & *11:02 & *58:01 & *13:02 & $0.012 \%$ & $0.012 \%$ & 0.069 & $99.836 \%$ \\
\hline 908 & *11:02 & *67:01 & *12:01 & $0.012 \%$ & $0.012 \%$ & 0.069 & $99.837 \%$ \\
\hline 908 & *24:02 & *07:02 & $\star 04: 06$ & $0.012 \%$ & $-0.054 \%$ & -0.017 & $99.838 \%$ \\
\hline 908 & *24:02 & *07:02 & *14:54 & $0.012 \%$ & $-0.060 \%$ & -0.017 & $99.838 \%$ \\
\hline 908 & *24:02 & *13:01 & *14:06 & $0.012 \%$ & $0.006 \%$ & 0.005 & $99.839 \%$ \\
\hline 908 & *24:02 & *15:01 & $\star 04: 04$ & $0.012 \%$ & $0.004 \%$ & 0.016 & $99.839 \%$ \\
\hline 908 & *24:02 & *15:01 & *14:03 & $0.012 \%$ & $-0.033 \%$ & -0.021 & $99.840 \%$ \\
\hline 908 & *24:02 & *15:01 & *14:05 & $0.012 \%$ & $-0.044 \%$ & -0.023 & $99.840 \%$ \\
\hline 908 & *24:02 & *15:01 & *14:29 & $0.012 \%$ & $0.011 \%$ & 0.486 & $99.840 \%$ \\
\hline 908 & *24:02 & *15:07 & *08:03 & $0.012 \%$ & $-0.007 \%$ & -0.012 & $99.841 \%$ \\
\hline 908 & *24:02 & *15:11 & *15:02 & $0.012 \%$ & $-0.026 \%$ & -0.028 & $99.842 \%$ \\
\hline 908 & *24:02 & *15:18 & *04:05 & $0.012 \%$ & $-0.059 \%$ & -0.043 & $99.842 \%$ \\
\hline 908 & *24:02 & $\star 15: 18$ & *15:01 & $0.012 \%$ & $-0.029 \%$ & -0.021 & $99.842 \%$ \\
\hline 908 & *24:02 & *15:27 & ${ }^{\star} 04: 05$ & $0.012 \%$ & $0.008 \%$ & 0.123 & $99.843 \%$ \\
\hline 908 & *24:02 & *27:04 & ${ }^{\star} 04: 03$ & $0.012 \%$ & $0.010 \%$ & 0.048 & $99.843 \%$ \\
\hline 908 & *24:02 & *27:04 & ${ }^{\star} 08: 03$ & $0.012 \%$ & $0.005 \%$ & 0.027 & $99.844 \%$ \\
\hline 908 & *24:02 & *27:05 & ${ }^{\star} 01: 01$ & $0.012 \%$ & $0.010 \%$ & 0.124 & $99.845 \%$ \\
\hline 908 & *24:02 & *35:01 & ${ }^{\star} 04: 07$ & $0.012 \%$ & $-0.003 \%$ & -0.006 & $99.845 \%$ \\
\hline 908 & *24:02 & *38:02 & *08:03 & $0.012 \%$ & $0.003 \%$ & 0.011 & $99.845 \%$ \\
\hline
\end{tabular}




\begin{tabular}{|c|c|c|c|c|c|c|c|}
\hline 908 & *24:02 & *39:01 & ${ }^{\star} 01: 01$ & $0.012 \%$ & $-0.061 \%$ & -0.018 & $99.846 \%$ \\
\hline 908 & *24:02 & *39:01 & *12:01 & $0.012 \%$ & $-0.034 \%$ & -0.010 & $99.846 \%$ \\
\hline 908 & *24:02 & *39:01 & *14:54 & $0.012 \%$ & $-0.032 \%$ & -0.009 & $99.847 \%$ \\
\hline 908 & *24:02 & *39:02 & ${ }^{\star} 09: 01$ & $0.012 \%$ & $-0.003 \%$ & -0.011 & $99.847 \%$ \\
\hline 908 & *24:02 & *40:01 & ${ }^{\star} 04: 10$ & $0.012 \%$ & $-0.029 \%$ & -0.014 & $99.847 \%$ \\
\hline 908 & *24:02 & *40:01 & ${ }^{\star} 07: 01$ & $0.012 \%$ & $0.003 \%$ & 0.008 & $99.848 \%$ \\
\hline 908 & *24:02 & *40:01 & *12:02 & $0.012 \%$ & $-0.023 \%$ & -0.013 & $99.848 \%$ \\
\hline 908 & *24:02 & *40:01 & *13:02 & $0.012 \%$ & $-0.095 \%$ & -0.018 & $99.849 \%$ \\
\hline 908 & *24:02 & *40:01 & *14:03 & $0.012 \%$ & $-0.019 \%$ & -0.012 & $99.849 \%$ \\
\hline 908 & *24:02 & *40:02 & *12:02 & $0.012 \%$ & $-0.041 \%$ & -0.023 & $99.849 \%$ \\
\hline 908 & *24:02 & *40:02 & *13:02 & $0.012 \%$ & $-0.149 \%$ & -0.028 & $99.849 \%$ \\
\hline 908 & *24:02 & *40:03 & ${ }^{*} 04: 06$ & $0.012 \%$ & $0.008 \%$ & 0.023 & $99.850 \%$ \\
\hline 908 & *24:02 & *40:03 & ${ }^{*} 08: 02$ & $0.012 \%$ & $0.006 \%$ & 0.019 & $99.850 \%$ \\
\hline 908 & *24:02 & *40:06 & ${ }^{*} 04: 06$ & $0.012 \%$ & $-0.041 \%$ & -0.013 & $99.851 \%$ \\
\hline 908 & *24:02 & *40:06 & *12:02 & $0.012 \%$ & $-0.018 \%$ & -0.010 & $99.851 \%$ \\
\hline 908 & *24:02 & *40:06 & *14:05 & $0.012 \%$ & $-0.021 \%$ & -0.011 & $99.852 \%$ \\
\hline 908 & *24:02 & *46:01 & *12:02 & $0.012 \%$ & $-0.020 \%$ & -0.011 & $99.852 \%$ \\
\hline 908 & *24:02 & *46:01 & ${ }^{\star} 13: 02$ & $0.012 \%$ & $-0.086 \%$ & -0.018 & $99.853 \%$ \\
\hline 908 & *24:02 & *46:01 & *14:03 & $0.012 \%$ & $-0.017 \%$ & -0.011 & $99.854 \%$ \\
\hline 908 & *24:02 & *48:01 & ${ }^{\star} 01: 01$ & $0.012 \%$ & $-0.048 \%$ & -0.017 & $99.854 \%$ \\
\hline 908 & *24:02 & *48:01 & ${ }^{\star} 08: 03$ & $0.012 \%$ & $-0.076 \%$ & -0.028 & $99.854 \%$ \\
\hline 908 & *24:02 & *48:01 & ${ }^{\star} 12: 02$ & $0.012 \%$ & $-0.007 \%$ & -0.004 & $99.855 \%$ \\
\hline 908 & *24:02 & *48:01 & ${ }^{\star} 14: 06$ & $0.012 \%$ & $-0.001 \%$ & -0.001 & $99.855 \%$ \\
\hline 908 & *24:02 & *51:02 & ${ }^{\star} 08: 02$ & $0.012 \%$ & $0.009 \%$ & 0.044 & $99.855 \%$ \\
\hline 908 & *24:02 & *51:02 & *12:01 & $0.012 \%$ & $0.009 \%$ & 0.046 & $99.856 \%$ \\
\hline 908 & *24:02 & $\star 52: 01$ & *14:03 & $0.012 \%$ & $-0.054 \%$ & -0.035 & $99.856 \%$ \\
\hline 908 & *24:02 & $\star 54: 01$ & ${ }^{*} 04: 01$ & $0.012 \%$ & $-0.013 \%$ & -0.015 & $99.856 \%$ \\
\hline 908 & *24:02 & $\star 54: 01$ & ${ }^{*} 04: 04$ & $0.012 \%$ & $0.004 \%$ & 0.017 & $99.857 \%$ \\
\hline 908 & *24:02 & $\star 54: 01$ & *10:01 & $0.012 \%$ & $-0.004 \%$ & -0.007 & $99.857 \%$ \\
\hline 908 & *24:02 & $\star 54: 01$ & *12:02 & $0.012 \%$ & $-0.038 \%$ & -0.021 & $99.857 \%$ \\
\hline 908 & *24:02 & $\star 54: 01$ & *13:02 & $0.012 \%$ & $-0.139 \%$ & -0.026 & $99.857 \%$ \\
\hline 908 & *24:02 & *54:01 & *14:54 & $0.012 \%$ & $-0.083 \%$ & -0.025 & $99.857 \%$ \\
\hline 908 & *24:02 & *54:01 & *16:02 & $0.012 \%$ & $-0.015 \%$ & -0.016 & $99.858 \%$ \\
\hline 908 & *24:02 & *55:02 & ${ }^{*} 12: 02$ & $0.012 \%$ & $-0.005 \%$ & -0.003 & $99.858 \%$ \\
\hline 908 & *24:02 & *55:02 & *14:05 & $0.012 \%$ & $-0.006 \%$ & -0.003 & $99.858 \%$ \\
\hline 908 & *24:02 & *56:01 & ${ }^{*} 08: 03$ & $0.012 \%$ & $-0.017 \%$ & -0.019 & $99.859 \%$ \\
\hline 908 & *24:02 & *56:01 & *11:01 & $0.012 \%$ & $0.002 \%$ & 0.003 & $99.859 \%$ \\
\hline 908 & *24:02 & *56:01 & ${ }^{\star} 12: 01$ & $0.012 \%$ & $-0.001 \%$ & -0.001 & $99.860 \%$ \\
\hline 908 & *24:02 & *56:03 & ${ }^{\star} 12: 01$ & $0.012 \%$ & $0.009 \%$ & 0.039 & $99.860 \%$ \\
\hline 908 & *24:02 & *67:01 & ${ }^{\star} 04: 05$ & $0.012 \%$ & $-0.045 \%$ & -0.041 & $99.861 \%$ \\
\hline 908 & *24:02 & *67:01 & *15:01 & $0.012 \%$ & $-0.021 \%$ & -0.018 & $99.861 \%$ \\
\hline 908 & *24:20 & *13:01 & ${ }^{\star} 12: 02$ & $0.012 \%$ & $0.011 \%$ & 0.017 & $99.861 \%$ \\
\hline 908 & *24:20 & *35:01 & ${ }^{\star} 04: 10$ & $0.012 \%$ & $0.010 \%$ & 0.015 & $99.862 \%$ \\
\hline 908 & *24:20 & *35:01 & ${ }^{\star} 08: 03$ & $0.012 \%$ & $0.007 \%$ & 0.010 & $99.862 \%$ \\
\hline 908 & *24:20 & $\star 35: 01$ & ${ }^{\star} 09: 01$ & $0.012 \%$ & $0.004 \%$ & 0.005 & $99.863 \%$ \\
\hline 908 & *24:20 & *39:01 & ${ }^{\star} 15: 01$ & $0.012 \%$ & $0.010 \%$ & 0.014 & $99.863 \%$ \\
\hline 908 & *24:20 & *40:01 & ${ }^{\star} 08: 02$ & $0.012 \%$ & $0.010 \%$ & 0.015 & $99.864 \%$ \\
\hline 908 & *24:20 & *40:01 & *11:01 & $0.012 \%$ & $0.011 \%$ & 0.015 & $99.864 \%$ \\
\hline 908 & *24:20 & *40:02 & *14:54 & $0.012 \%$ & $0.010 \%$ & 0.014 & $99.865 \%$ \\
\hline 908 & *24:20 & *40:06 & ${ }^{*} 09: 01$ & $0.012 \%$ & $0.007 \%$ & 0.010 & $99.865 \%$ \\
\hline 908 & *24:20 & *46:01 & ${ }^{*} 09: 01$ & $0.012 \%$ & $0.007 \%$ & 0.010 & $99.866 \%$ \\
\hline 908 & *24:20 & *55:02 & *15:01 & $0.012 \%$ & $0.010 \%$ & 0.015 & $99.866 \%$ \\
\hline 908 & *24:25 & *15:01 & *09:01 & $0.012 \%$ & $0.011 \%$ & 0.999 & $99.866 \%$ \\
\hline 908 & *24:88 & *55:02 & ${ }^{*} 09: 01$ & $0.012 \%$ & $0.012 \%$ & 1.000 & $99.867 \%$ \\
\hline
\end{tabular}




\begin{tabular}{|c|c|c|c|c|c|c|c|}
\hline 908 & *26:01 & \begin{tabular}{|l|}
$* 15: 01$ \\
\end{tabular} & *14:05 & $0.012 \%$ & $0.000 \%$ & 0.000 & $99.867 \%$ \\
\hline 908 & *26:01 & $\star 15: 02$ & $\star 12: 02$ & $0.012 \%$ & $0.012 \%$ & 0.249 & $99.868 \%$ \\
\hline 908 & *26:01 & *15:07 & *04:05 & $0.012 \%$ & $0.005 \%$ & 0.009 & $99.868 \%$ \\
\hline 908 & *26:01 & *15:11 & ${ }^{\star} 04: 03$ & $0.012 \%$ & $0.010 \%$ & 0.010 & $99.869 \%$ \\
\hline 908 & *26:01 & *15:18 & *04:03 & $0.012 \%$ & $0.009 \%$ & 0.006 & $99.870 \%$ \\
\hline 908 & *26:01 & *15:18 & *08:02 & $0.012 \%$ & $0.007 \%$ & 0.005 & $99.870 \%$ \\
\hline 908 & *26:01 & *35:01 & ${ }^{\star} 01: 01$ & $0.012 \%$ & $-0.025 \%$ & -0.004 & $99.871 \%$ \\
\hline 908 & *26:01 & *35:01 & *14:05 & $0.012 \%$ & $-0.001 \%$ & 0.000 & $99.871 \%$ \\
\hline 908 & *26:01 & *35:01 & *15:02 & $0.012 \%$ & $-0.055 \%$ & -0.007 & $99.871 \%$ \\
\hline 908 & *26:01 & *39:01 & ${ }^{\star} 04: 03$ & $0.012 \%$ & $0.004 \%$ & 0.002 & $99.872 \%$ \\
\hline 908 & *26:01 & *39:01 & *09:01 & $0.012 \%$ & $-0.026 \%$ & -0.008 & $99.872 \%$ \\
\hline 908 & *26:01 & *39:01 & *11:01 & $0.012 \%$ & $0.004 \%$ & 0.002 & $99.873 \%$ \\
\hline 908 & *26:01 & *40:01 & *14:54 & $0.012 \%$ & $-0.003 \%$ & -0.001 & $99.873 \%$ \\
\hline 908 & *26:01 & *40:01 & *15:02 & $0.012 \%$ & $-0.032 \%$ & -0.006 & $99.873 \%$ \\
\hline 908 & *26:01 & $\star 40: 02$ & ${ }^{\star} 04: 07$ & $0.012 \%$ & $0.009 \%$ & 0.017 & $99.873 \%$ \\
\hline 908 & *26:01 & $\star 40: 02$ & *14:06 & $0.012 \%$ & $0.004 \%$ & 0.003 & $99.874 \%$ \\
\hline 908 & *26:01 & *40:06 & *11:01 & $0.012 \%$ & $0.002 \%$ & 0.001 & $99.874 \%$ \\
\hline 908 & *26:01 & $* 40: 06$ & $\star 12: 02$ & $0.012 \%$ & $0.005 \%$ & 0.003 & $99.875 \%$ \\
\hline 908 & *26:01 & $* 40: 06$ & *14:54 & $0.012 \%$ & $-0.001 \%$ & 0.000 & $99.875 \%$ \\
\hline 908 & *26:01 & *48:01 & *08:03 & $0.012 \%$ & $-0.007 \%$ & -0.003 & $99.875 \%$ \\
\hline 908 & *26:01 & *51:01 & *04:03 & $0.012 \%$ & $-0.008 \%$ & -0.003 & $99.876 \%$ \\
\hline 908 & *26:01 & *51:01 & *04:10 & $0.012 \%$ & $-0.003 \%$ & -0.002 & $99.876 \%$ \\
\hline 908 & *26:01 & *51:01 & *12:01 & $0.012 \%$ & $-0.014 \%$ & -0.004 & $99.876 \%$ \\
\hline 908 & *26:01 & *51:01 & *14:54 & $0.012 \%$ & $-0.013 \%$ & -0.004 & $99.876 \%$ \\
\hline 908 & *26:01 & *51:01 & *14:05 & $0.012 \%$ & $-0.003 \%$ & -0.001 & $99.877 \%$ \\
\hline 908 & *26:01 & $\star 54: 01$ & *16:02 & $0.012 \%$ & $0.006 \%$ & 0.006 & $99.877 \%$ \\
\hline 908 & *26:01 & $\star 55: 02$ & *04:03 & $0.012 \%$ & $0.006 \%$ & 0.003 & $99.878 \%$ \\
\hline 908 & *26:01 & *67:01 & *15:01 & $0.012 \%$ & $0.005 \%$ & 0.004 & $99.878 \%$ \\
\hline 908 & *26:01 & *67:01 & *16:02 & $0.012 \%$ & $0.011 \%$ & 0.011 & $99.879 \%$ \\
\hline 908 & *26:02 & *15:01 & *15:02 & $0.012 \%$ & $-0.004 \%$ & -0.002 & $99.879 \%$ \\
\hline 908 & *26:02 & *35:01 & ${ }^{\star} 04: 10$ & $0.012 \%$ & $0.008 \%$ & 0.005 & $99.880 \%$ \\
\hline 908 & *26:02 & *35:01 & ${ }^{\star} 08: 02$ & $0.012 \%$ & $0.005 \%$ & 0.003 & $99.880 \%$ \\
\hline 908 & *26:02 & *35:01 & ${ }^{\star} 09: 01$ & $0.012 \%$ & $-0.010 \%$ & -0.005 & $99.880 \%$ \\
\hline 908 & *26:02 & *39:01 & ${ }^{\star} 08: 03$ & $0.012 \%$ & $0.006 \%$ & 0.003 & $99.881 \%$ \\
\hline 908 & *26:02 & *40:01 & *04:05 & $0.012 \%$ & $-0.002 \%$ & -0.001 & $99.881 \%$ \\
\hline 908 & *26:02 & *40:01 & *12:01 & $0.012 \%$ & $0.008 \%$ & 0.004 & $99.882 \%$ \\
\hline 908 & *26:02 & *40:02 & ${ }^{\star} 04: 05$ & $0.012 \%$ & $-0.009 \%$ & -0.005 & $99.883 \%$ \\
\hline 908 & *26:02 & *40:02 & ${ }^{*} 04: 06$ & $0.012 \%$ & $0.007 \%$ & 0.004 & $99.883 \%$ \\
\hline 908 & *26:02 & *40:02 & *04:10 & $0.012 \%$ & $0.008 \%$ & 0.005 & $99.884 \%$ \\
\hline 908 & *26:02 & *40:02 & *15:01 & $0.012 \%$ & $0.000 \%$ & 0.000 & $99.884 \%$ \\
\hline 908 & *26:02 & *40:06 & ${ }^{\star} 01: 01$ & $0.012 \%$ & $0.007 \%$ & 0.004 & $99.885 \%$ \\
\hline 908 & *26:02 & *40:06 & *14:54 & $0.012 \%$ & $0.009 \%$ & 0.005 & $99.885 \%$ \\
\hline 908 & *26:02 & *40:06 & *14:05 & $0.012 \%$ & $0.010 \%$ & 0.005 & $99.886 \%$ \\
\hline 908 & *26:02 & *55:02 & ${ }^{\star} 09: 01$ & $0.012 \%$ & $0.005 \%$ & 0.003 & $99.886 \%$ \\
\hline 908 & *26:03 & ${ }^{*} 07: 02$ & ${ }^{\star} 01: 01$ & $0.012 \%$ & $0.004 \%$ & 0.002 & $99.886 \%$ \\
\hline 908 & *26:03 & *15:01 & ${ }^{\star} 01: 01$ & $0.012 \%$ & $0.001 \%$ & 0.001 & $99.887 \%$ \\
\hline 908 & *26:03 & *15:01 & ${ }^{\star} 04: 10$ & $0.012 \%$ & $0.008 \%$ & 0.004 & $99.887 \%$ \\
\hline 908 & *26:03 & $\star 15: 01$ & *11:01 & $0.012 \%$ & $0.007 \%$ & 0.003 & $99.888 \%$ \\
\hline 908 & *26:03 & $\star 35: 01$ & ${ }^{\star} 01: 01$ & $0.012 \%$ & $0.001 \%$ & 0.000 & $99.888 \%$ \\
\hline 908 & *26:03 & $\star 35: 01$ & ${ }^{\star} 04: 06$ & $0.012 \%$ & $0.006 \%$ & 0.003 & $99.888 \%$ \\
\hline 908 & *26:03 & $\star 35: 01$ & *12:01 & $0.012 \%$ & $0.005 \%$ & 0.002 & $99.889 \%$ \\
\hline 908 & *26:03 & $\star 35: 01$ & *14:54 & $0.012 \%$ & $0.005 \%$ & 0.002 & $99.889 \%$ \\
\hline 908 & $\star 26: 03$ & $\star 39: 01$ & ${ }^{\star} 04: 03$ & $0.012 \%$ & $0.009 \%$ & 0.004 & $99.890 \%$ \\
\hline 908 & $\star 26: 03$ & $\star 39: 01$ & ${ }^{\star} 04: 05$ & $0.012 \%$ & $0.001 \%$ & 0.000 & $99.890 \%$ \\
\hline
\end{tabular}




\begin{tabular}{|c|c|c|c|c|c|c|c|}
\hline 908 & *26:03 & *39:01 & ${ }^{\star} 09: 01$ & $0.012 \%$ & $0.000 \%$ & 0.000 & $99.891 \%$ \\
\hline 908 & *26:03 & $\star 39: 01$ & *15:01 & $0.012 \%$ & $0.006 \%$ & 0.002 & $99.891 \%$ \\
\hline 908 & *26:03 & $\star 40: 02$ & $\star^{*} 04: 05$ & $0.012 \%$ & $-0.013 \%$ & -0.006 & $99.891 \%$ \\
\hline 908 & $\star 26: 03$ & *40:02 & ${ }^{\star} 04: 10$ & $0.012 \%$ & $0.008 \%$ & 0.004 & $99.892 \%$ \\
\hline 908 & $\star 26: 03$ & *40:02 & ${ }^{\star} 08: 02$ & $0.012 \%$ & $0.004 \%$ & 0.002 & $99.892 \%$ \\
\hline 908 & *26:03 & *40:02 & ${ }^{\star} 09: 01$ & $0.012 \%$ & $-0.015 \%$ & -0.007 & $99.893 \%$ \\
\hline 908 & *26:03 & *48:01 & ${ }^{*} 09: 01$ & $0.012 \%$ & $0.002 \%$ & 0.001 & $99.893 \%$ \\
\hline 908 & *26:03 & *51:01 & ${ }^{*} 08: 02$ & $0.012 \%$ & $0.003 \%$ & 0.001 & $99.894 \%$ \\
\hline 908 & *26:03 & $\star 51: 01$ & ${ }^{*} 09: 01$ & $0.012 \%$ & $-0.019 \%$ & -0.008 & $99.894 \%$ \\
\hline 908 & *26:03 & $\star 55: 02$ & ${ }^{*} 09: 01$ & $0.012 \%$ & $0.004 \%$ & 0.002 & $99.894 \%$ \\
\hline 908 & *26:03 & *56:01 & ${ }^{*} 09: 01$ & $0.012 \%$ & $0.009 \%$ & 0.009 & $99.894 \%$ \\
\hline 908 & *30:04 & *14:01 & ${ }^{*} 04: 04$ & $0.012 \%$ & $0.012 \%$ & 0.498 & $99.896 \%$ \\
\hline 908 & *31:01 & *15:07 & ${ }^{*} 04: 03$ & $0.012 \%$ & $0.010 \%$ & 0.017 & $99.896 \%$ \\
\hline 908 & *31:01 & *15:08 & *08:02 & $0.012 \%$ & $0.012 \%$ & 1.000 & $99.897 \%$ \\
\hline 908 & *31:01 & *15:11 & ${ }^{*} 04: 05$ & $0.012 \%$ & $0.000 \%$ & 0.000 & $99.897 \%$ \\
\hline 908 & *31:01 & *15:18 & ${ }^{*} 04: 03$ & $0.012 \%$ & $0.008 \%$ & 0.006 & $99.898 \%$ \\
\hline 908 & *31:01 & *15:18 & ${ }^{*} 04: 05$ & $0.012 \%$ & $-0.006 \%$ & -0.004 & $99.898 \%$ \\
\hline 908 & *31:01 & *15:38 & *11:01 & $0.012 \%$ & $0.012 \%$ & 0.666 & $99.899 \%$ \\
\hline 908 & *31:01 & *27:04 & ${ }^{\star} 08: 03$ & $0.012 \%$ & $0.010 \%$ & 0.050 & $99.899 \%$ \\
\hline 908 & *31:01 & *27:06 & ${ }^{\star} 08: 03$ & $0.012 \%$ & $0.012 \%$ & 1.004 & $99.900 \%$ \\
\hline 908 & *31:01 & $\star 37: 01$ & *15:01 & $0.012 \%$ & $0.007 \%$ & 0.011 & $99.900 \%$ \\
\hline 908 & *31:01 & $\star 38: 02$ & ${ }^{\star} 08: 03$ & $0.012 \%$ & $0.009 \%$ & 0.034 & $99.901 \%$ \\
\hline 908 & *31:01 & $\star 38: 02$ & ${ }^{\star} 09: 01$ & $0.012 \%$ & $0.008 \%$ & 0.029 & $99.901 \%$ \\
\hline 908 & *31:01 & *39:01 & ${ }^{\star} 09: 01$ & $0.012 \%$ & $-0.032 \%$ & -0.009 & $99.901 \%$ \\
\hline 908 & *31:01 & *39:01 & *12:01 & $0.012 \%$ & $0.001 \%$ & 0.000 & $99.902 \%$ \\
\hline 908 & *31:01 & *39:02 & ${ }^{*} 01: 01$ & $0.012 \%$ & $0.010 \%$ & 0.037 & $99.903 \%$ \\
\hline 908 & *31:01 & *39:04 & ${ }^{*} 04: 03$ & $0.012 \%$ & $0.011 \%$ & 0.053 & $99.903 \%$ \\
\hline 908 & *31:01 & *40:01 & ${ }^{*} 04: 10$ & $0.012 \%$ & $0.002 \%$ & 0.001 & $99.904 \%$ \\
\hline 908 & *31:01 & *40:01 & ${ }^{*} 08: 02$ & $0.012 \%$ & $-0.009 \%$ & -0.002 & $99.904 \%$ \\
\hline 908 & *31:01 & *40:01 & *12:02 & $0.012 \%$ & $0.003 \%$ & 0.002 & $99.904 \%$ \\
\hline 908 & *31:01 & *40:02 & ${ }^{*} 04: 01$ & $0.012 \%$ & $0.005 \%$ & 0.006 & $99.905 \%$ \\
\hline 908 & *31:01 & *40:02 & *12:05 & $0.012 \%$ & $0.011 \%$ & 0.663 & $99.905 \%$ \\
\hline 908 & *31:01 & *40:02 & ${ }^{*} 14: 03$ & $0.012 \%$ & $0.000 \%$ & 0.000 & $99.905 \%$ \\
\hline 908 & *31:01 & *40:02 & *15:02 & $0.012 \%$ & $-0.065 \%$ & -0.008 & $99.905 \%$ \\
\hline 908 & *31:01 & $* 40: 03$ & ${ }^{*} 04: 10$ & $0.012 \%$ & $0.011 \%$ & 0.032 & $99.906 \%$ \\
\hline 908 & *31:01 & $* 40: 06$ & ${ }^{*} 04: 05$ & $0.012 \%$ & $-0.043 \%$ & -0.010 & $99.907 \%$ \\
\hline 908 & *31:01 & $\star 40: 06$ & ${ }^{\star} 04: 06$ & $0.012 \%$ & $-0.001 \%$ & 0.000 & $99.907 \%$ \\
\hline 908 & *31:01 & $\star 40: 06$ & *15:01 & $0.012 \%$ & $-0.020 \%$ & -0.004 & $99.907 \%$ \\
\hline 908 & *31:01 & $\star 46: 01$ & ${ }^{\star} 12: 01$ & $0.012 \%$ & $-0.004 \%$ & -0.001 & $99.908 \%$ \\
\hline 908 & *31:01 & $\star 46: 01$ & ${ }^{\star} 15: 02$ & $0.012 \%$ & $-0.035 \%$ & -0.007 & $99.908 \%$ \\
\hline 908 & *31:01 & *48:01 & ${ }^{\star} 04: 04$ & $0.012 \%$ & $0.011 \%$ & 0.041 & $99.909 \%$ \\
\hline 908 & *31:01 & *48:01 & ${ }^{\star} 04: 07$ & $0.012 \%$ & $0.010 \%$ & 0.021 & $99.910 \%$ \\
\hline 908 & *31:01 & *48:01 & ${ }^{\star} 04: 10$ & $0.012 \%$ & $0.006 \%$ & 0.003 & $99.910 \%$ \\
\hline 908 & *31:01 & $\star 52: 01$ & ${ }^{\star} 04: 03$ & $0.012 \%$ & $-0.016 \%$ & -0.006 & $99.911 \%$ \\
\hline 908 & *31:01 & $\star 52: 01$ & *14:54 & $0.012 \%$ & $-0.023 \%$ & -0.007 & $99.911 \%$ \\
\hline 908 & *31:01 & $\star 54: 01$ & ${ }^{\star} 01: 01$ & $0.012 \%$ & $-0.028 \%$ & -0.005 & $99.912 \%$ \\
\hline 908 & *31:01 & *54:01 & ${ }^{\star} 08: 02$ & $0.012 \%$ & $-0.017 \%$ & -0.004 & $99.912 \%$ \\
\hline 908 & *31:01 & $\star 54: 01$ & *13:02 & $0.012 \%$ & $-0.026 \%$ & -0.005 & $99.912 \%$ \\
\hline 908 & *31:01 & $\star 54: 01$ & *14:05 & $0.012 \%$ & $-0.002 \%$ & -0.001 & $99.913 \%$ \\
\hline 908 & *31:01 & $\star 55: 02$ & ${ }^{*} 04: 01$ & $0.012 \%$ & $0.010 \%$ & 0.011 & $99.913 \%$ \\
\hline 908 & *31:01 & $\star 55: 02$ & *15:02 & $0.012 \%$ & $-0.012 \%$ & -0.005 & $99.914 \%$ \\
\hline 908 & *31:01 & $\star 55: 04$ & ${ }^{*} 09: 01$ & $0.012 \%$ & $0.010 \%$ & 0.076 & $99.914 \%$ \\
\hline 908 & *31:01 & $\star 56: 01$ & *14:03 & $0.012 \%$ & $0.010 \%$ & 0.011 & $99.915 \%$ \\
\hline 908 & *32:01 & *44:02 & ${ }^{*} 04: 05$ & $0.012 \%$ & $0.012 \%$ & 0.332 & $99.915 \%$ \\
\hline
\end{tabular}




\begin{tabular}{|c|c|c|c|c|c|c|c|}
\hline 908 & *33:01 & \begin{tabular}{|l|} 
*14:02 \\
\end{tabular} & ${ }^{\star} 01: 02$ & $0.012 \%$ & $0.012 \%$ & 0.664 & $99.916 \%$ \\
\hline 908 & *33:03 & $* 07: 02$ & *13:02 & $0.012 \%$ & $-0.010 \%$ & -0.002 & $99.917 \%$ \\
\hline 908 & *33:03 & $\star 15: 18$ & *15:01 & $0.012 \%$ & $0.004 \%$ & 0.003 & $99.918 \%$ \\
\hline 908 & *33:03 & $\star 27: 04$ & ${ }^{\star} 08: 03$ & $0.012 \%$ & $0.010 \%$ & 0.051 & $99.918 \%$ \\
\hline 908 & *33:03 & *35:01 & *15:01 & $0.012 \%$ & $-0.032 \%$ & -0.005 & $99.919 \%$ \\
\hline 908 & *33:03 & *37:01 & *10:01 & $0.012 \%$ & $0.011 \%$ & 0.020 & $99.919 \%$ \\
\hline 908 & *33:03 & *39:01 & ${ }^{*} 08: 03$ & $0.012 \%$ & $-0.009 \%$ & -0.003 & $99.920 \%$ \\
\hline 908 & *33:03 & *39:01 & *15:01 & $0.012 \%$ & $-0.007 \%$ & -0.002 & $99.920 \%$ \\
\hline 908 & *33:03 & *40:01 & ${ }^{\star} 04: 05$ & $0.012 \%$ & $-0.039 \%$ & -0.007 & $99.921 \%$ \\
\hline 908 & *33:03 & *40:01 & *12:01 & $0.012 \%$ & $-0.002 \%$ & -0.001 & $99.921 \%$ \\
\hline 908 & *33:03 & *40:01 & *15:02 & $0.012 \%$ & $-0.028 \%$ & -0.005 & $99.921 \%$ \\
\hline 908 & *33:03 & *40:02 & *12:01 & $0.012 \%$ & $-0.009 \%$ & -0.002 & $99.922 \%$ \\
\hline 908 & *33:03 & *40:02 & $\star 14: 54$ & $0.012 \%$ & $-0.008 \%$ & -0.002 & $99.922 \%$ \\
\hline 908 & *33:03 & *40:02 & $\star 15: 01$ & $0.012 \%$ & $-0.032 \%$ & -0.005 & $99.923 \%$ \\
\hline 908 & *33:03 & *40:06 & $\star 15: 01$ & $0.012 \%$ & $-0.013 \%$ & -0.003 & $99.923 \%$ \\
\hline 908 & *33:03 & *44:03 & $\star^{\star} 04: 06$ & $0.012 \%$ & $-0.002 \%$ & -0.001 & $99.923 \%$ \\
\hline 908 & *33:03 & *44:03 & *14:03 & $0.012 \%$ & $0.005 \%$ & 0.003 & $99.924 \%$ \\
\hline 908 & *33:03 & *44:03 & *14:07 & $0.012 \%$ & $0.011 \%$ & 0.121 & $99.924 \%$ \\
\hline 908 & *33:03 & *46:01 & ${ }^{\star} 01: 01$ & $0.012 \%$ & $-0.008 \%$ & -0.002 & $99.925 \%$ \\
\hline 908 & *33:03 & *46:01 & *04:05 & $0.012 \%$ & $-0.035 \%$ & -0.007 & $99.925 \%$ \\
\hline 908 & *33:03 & *48:01 & *15:01 & $0.012 \%$ & $-0.004 \%$ & -0.001 & $99.925 \%$ \\
\hline 908 & *33:03 & *51:01 & ${ }^{*} 04: 04$ & $0.012 \%$ & $0.010 \%$ & 0.037 & $99.926 \%$ \\
\hline 908 & *33:03 & *51:01 & *13:01 & $0.012 \%$ & $0.008 \%$ & 0.014 & $99.926 \%$ \\
\hline 908 & *33:03 & *51:01 & *16:02 & $0.012 \%$ & $0.005 \%$ & 0.005 & $99.927 \%$ \\
\hline 908 & *33:03 & *55:02 & *14:05 & $0.012 \%$ & $0.008 \%$ & 0.004 & $99.927 \%$ \\
\hline 908 & *33:03 & $\star 56: 01$ & *09:01 & $0.012 \%$ & $0.002 \%$ & 0.003 & $99.928 \%$ \\
\hline 908 & *33:03 & *58:01 & ${ }^{*} 04: 01$ & $0.012 \%$ & $0.011 \%$ & 0.018 & $99.928 \%$ \\
\hline 908 & *33:03 & $\star 58: 01$ & *13:01 & $0.012 \%$ & $0.011 \%$ & 0.020 & $99.929 \%$ \\
\hline 908 & *33:03 & *58:01 & *15:01 & $0.012 \%$ & $0.008 \%$ & 0.013 & $99.930 \%$ \\
\hline 908 & *33:03 & *59:01 & ${ }^{*} 04: 05$ & $0.012 \%$ & $-0.007 \%$ & -0.003 & $99.930 \%$ \\
\hline 1270 & ${ }^{\star} 01: 01$ & ${ }^{\star} 08: 01$ & ${ }^{*} 07: 01$ & $0.006 \%$ & $0.006 \%$ & 1.005 & $99.931 \%$ \\
\hline 1270 & ${ }^{\star} 01: 01$ & *15:01 & ${ }^{*} 09: 01$ & $0.006 \%$ & $0.001 \%$ & 0.001 & $99.931 \%$ \\
\hline 1270 & ${ }^{*} 01: 01$ & *35:01 & ${ }^{\star} 09: 01$ & $0.006 \%$ & $0.000 \%$ & 0.001 & $99.931 \%$ \\
\hline 1270 & ${ }^{\star} 01: 01$ & *35:01 & *13:02 & $0.006 \%$ & $0.004 \%$ & 0.008 & $99.931 \%$ \\
\hline 1270 & ${ }^{\star} 01: 01$ & *35:05 & *12:02 & $0.006 \%$ & $0.006 \%$ & 0.502 & $99.932 \%$ \\
\hline 1270 & ${ }^{\star} 01: 01$ & *37:01 & ${ }^{\star} 04: 03$ & $0.006 \%$ & $0.006 \%$ & 0.012 & $99.932 \%$ \\
\hline 1270 & ${ }^{\star} 01: 01$ & *37:01 & *08:03 & $0.006 \%$ & $0.006 \%$ & 0.012 & $99.932 \%$ \\
\hline 1270 & ${ }^{\star} 01: 01$ & *37:01 & *11:01 & $0.006 \%$ & $0.006 \%$ & 0.012 & $99.933 \%$ \\
\hline 1270 & ${ }^{\star} 01: 01$ & *37:01 & $\star 12: 02$ & $0.006 \%$ & $0.006 \%$ & 0.012 & $99.933 \%$ \\
\hline 1270 & ${ }^{\star} 01: 01$ & *37:01 & $\star 13: 01$ & $0.006 \%$ & $0.006 \%$ & 0.012 & $99.934 \%$ \\
\hline 1270 & *01:01 & *37:01 & *14:03 & $0.006 \%$ & $0.006 \%$ & 0.012 & $99.934 \%$ \\
\hline 1270 & *01:01 & $\star 37: 01$ & *14:06 & $0.006 \%$ & $0.006 \%$ & 0.012 & $99.934 \%$ \\
\hline 1270 & ${ }^{*} 01: 01$ & *37:01 & *15:01 & $0.006 \%$ & $0.006 \%$ & 0.012 & $99.934 \%$ \\
\hline 1270 & ${ }^{\star} 01: 01$ & $\star 39: 01$ & ${ }^{\star} 08: 02$ & $0.006 \%$ & $0.005 \%$ & 0.011 & $99.935 \%$ \\
\hline 1270 & ${ }^{\star} 01: 01$ & $\star 40: 02$ & ${ }^{\star} 04: 05$ & $0.006 \%$ & $0.001 \%$ & 0.002 & $99.935 \%$ \\
\hline 1270 & ${ }^{\star} 01: 01$ & $\star 40: 02$ & *11:01 & $0.006 \%$ & $0.005 \%$ & 0.010 & $99.935 \%$ \\
\hline 1270 & ${ }^{\star} 01: 01$ & $\star 40: 06$ & ${ }^{\star} 09: 01$ & $0.006 \%$ & $0.003 \%$ & 0.006 & $99.935 \%$ \\
\hline 1270 & ${ }^{\star} 01: 01$ & $\star 54: 01$ & ${ }^{\star} 04: 05$ & $0.006 \%$ & $0.001 \%$ & 0.002 & $99.935 \%$ \\
\hline 1270 & ${ }^{\star} 01: 01$ & $\star 56: 01$ & ${ }^{\star} 09: 01$ & $0.006 \%$ & $0.005 \%$ & 0.011 & $99.935 \%$ \\
\hline 1270 & ${ }^{\star} 01: 01$ & $\star 57: 01$ & ${ }^{\star} 07: 01$ & $0.006 \%$ & $0.006 \%$ & 1.005 & $99.936 \%$ \\
\hline 1270 & ${ }^{\star} 01: 01$ & *58:01 & ${ }^{\star} 03: 01$ & $0.006 \%$ & $0.006 \%$ & 0.048 & $99.936 \%$ \\
\hline 1270 & ${ }^{\star} 02: 01$ & ${ }^{\star} 07: 02$ & ${ }^{\star} 04: 01$ & $0.006 \%$ & $0.000 \%$ & 0.000 & $99.936 \%$ \\
\hline 1270 & ${ }^{\star} 02: 01$ & ${ }^{\star} 07: 02$ & ${ }^{\star} 04: 05$ & $0.006 \%$ & $-0.084 \%$ & -0.015 & $99.937 \%$ \\
\hline 1270 & ${ }^{\star} 02: 01$ & ${ }^{\star} 07: 02$ & ${ }^{*} 09: 01$ & $0.006 \%$ & $-0.089 \%$ & -0.016 & $99.937 \%$ \\
\hline
\end{tabular}




\begin{tabular}{|c|c|c|c|c|c|c|c|}
\hline 1270 & ${ }^{\star} 02: 01$ & *07:02 & *14:03 & $0.006 \%$ & $-0.005 \%$ & -0.003 & $99.937 \%$ \\
\hline 1270 & ${ }^{\star} 02: 01$ & *07:02 & *15:01 & $0.006 \%$ & $-0.045 \%$ & -0.008 & $99.937 \%$ \\
\hline 1270 & ${ }^{\star} 02: 01$ & *13:01 & ${ }^{\star} 08: 03$ & $0.006 \%$ & $-0.006 \%$ & -0.005 & $99.937 \%$ \\
\hline 1270 & ${ }^{\star} 02: 01$ & *13:01 & *15:01 & $0.006 \%$ & $-0.005 \%$ & -0.004 & $99.937 \%$ \\
\hline 1270 & ${ }^{\star} 02: 01$ & *13:01 & ${ }^{\star} 15: 02$ & $0.006 \%$ & $-0.009 \%$ & -0.008 & $99.938 \%$ \\
\hline 1270 & ${ }^{\star} 02: 01$ & *14:02 & ${ }^{\star} 01: 02$ & $0.006 \%$ & $0.006 \%$ & 0.251 & $99.938 \%$ \\
\hline 1270 & ${ }^{\star} 02: 01$ & *15:01 & ${ }^{*} 04: 04$ & $0.006 \%$ & $0.003 \%$ & 0.013 & $99.938 \%$ \\
\hline 1270 & ${ }^{*} 02: 01$ & *15:01 & *14:07 & $0.006 \%$ & $0.005 \%$ & 0.054 & $99.938 \%$ \\
\hline 1270 & ${ }^{*} 02: 01$ & *15:01 & *15:02 & $0.006 \%$ & $-0.091 \%$ & -0.012 & $99.938 \%$ \\
\hline 1270 & ${ }^{*} 02: 01$ & *15:04 & *14:02 & $0.006 \%$ & $0.006 \%$ & 1.005 & $99.939 \%$ \\
\hline 1270 & *02:01 & *15:07 & ${ }^{*} 09: 01$ & $0.006 \%$ & $-0.004 \%$ & -0.007 & $99.939 \%$ \\
\hline 1270 & ${ }^{*} 02: 01$ & *15:07 & *11:01 & $0.006 \%$ & $0.004 \%$ & 0.007 & $99.939 \%$ \\
\hline 1270 & ${ }^{*} 02: 01$ & *15:07 & *13:02 & $0.006 \%$ & $0.002 \%$ & 0.003 & $99.939 \%$ \\
\hline 1270 & ${ }^{*} 02: 01$ & *15:11 & ${ }^{*} 04: 01$ & $0.006 \%$ & $0.005 \%$ & 0.005 & $99.939 \%$ \\
\hline 1270 & *02:01 & *15:11 & ${ }^{*} 04: 07$ & $0.006 \%$ & $0.005 \%$ & 0.010 & $99.940 \%$ \\
\hline 1270 & ${ }^{*} 02: 01$ & *15:11 & *12:02 & $0.006 \%$ & $0.004 \%$ & 0.004 & $99.940 \%$ \\
\hline 1270 & ${ }^{*} 02: 01$ & *15:11 & *14:03 & $0.006 \%$ & $0.004 \%$ & 0.004 & $99.940 \%$ \\
\hline 1270 & *02:01 & *15:18 & ${ }^{\star} 01: 01$ & $0.006 \%$ & $-0.004 \%$ & - & $99.940 \%$ \\
\hline 1270 & ${ }^{\star} 02: 01$ & *15:18 & ${ }^{\star} 04: 06$ & $0.006 \%$ & $0.000 \%$ & 0.000 & $99.940 \%$ \\
\hline 1270 & ${ }^{\star} 02: 01$ & *15:18 & *14:03 & $0.006 \%$ & $0.003 \%$ & 0.002 & $99.940 \%$ \\
\hline 1270 & ${ }^{\star} 02: 01$ & *15:18 & *14:05 & $0.006 \%$ & $0.002 \%$ & 0.002 & $99.941 \%$ \\
\hline 1270 & ${ }^{\star} 02: 01$ & *15:27 & ${ }^{\star} 09: 01$ & $0.006 \%$ & $0.005 \%$ & 0.068 & $99.941 \%$ \\
\hline 1270 & ${ }^{\star} 02: 01$ & *18:01 & ${ }^{\star} 04: 03$ & $0.006 \%$ & $0.006 \%$ & 1.008 & $99.941 \%$ \\
\hline 1270 & ${ }^{\star} 02: 01$ & *27:04 & *11:01 & $0.006 \%$ & $0.005 \%$ & 0.025 & $99.941 \%$ \\
\hline 1270 & ${ }^{*} 02: 01$ & *27:04 & *12:02 & $0.006 \%$ & $0.005 \%$ & 0.026 & $99.941 \%$ \\
\hline 1270 & $\star 02: 01$ & $\star 35: 01$ & ${ }^{*} 04: 01$ & $0.006 \%$ & $-0.003 \%$ & -0.003 & $99.941 \%$ \\
\hline 1270 & $\star 02: 01$ & *35:01 & ${ }^{*} 04: 04$ & $0.006 \%$ & $0.003 \%$ & 0.013 & $99.941 \%$ \\
\hline 1270 & ${ }^{\star} 02: 01$ & *35:01 & *14:02 & $0.006 \%$ & $0.005 \%$ & 0.083 & $99.942 \%$ \\
\hline 1270 & ${ }^{\star} 02: 01$ & *35:01 & *14:05 & $0.006 \%$ & $-0.013 \%$ & -0.007 & $99.942 \%$ \\
\hline 1270 & ${ }^{\star} 02: 01$ & *35:01 & *15:02 & $0.006 \%$ & $-0.095 \%$ & -0.012 & $99.942 \%$ \\
\hline 1270 & ${ }^{*} 02: 01$ & $\star 35: 35$ & ${ }^{*} 08: 03$ & $0.006 \%$ & $0.006 \%$ & 0.998 & $99.942 \%$ \\
\hline 1270 & *02:01 & *37:01 & ${ }^{\star} 01: 01$ & $0.006 \%$ & $0.001 \%$ & 0.002 & $99.942 \%$ \\
\hline 1270 & ${ }^{*} 02: 01$ & *38:02 & *13:01 & $0.006 \%$ & $0.006 \%$ & 0.020 & $99.942 \%$ \\
\hline 1270 & *02:01 & *38:02 & *15:01 & $0.006 \%$ & $0.003 \%$ & 0.012 & $99.942 \%$ \\
\hline 1270 & *02:01 & *39:01 & ${ }^{*} 04: 03$ & $0.006 \%$ & $-0.005 \%$ & -0.002 & $99.943 \%$ \\
\hline 1270 & *02:01 & *39:01 & *11:01 & $0.006 \%$ & $-0.005 \%$ & -0.002 & $99.943 \%$ \\
\hline 1270 & *02:01 & *39:01 & *12:01 & $0.006 \%$ & $-0.009 \%$ & -0.003 & $99.943 \%$ \\
\hline 1270 & ${ }^{\star} 02: 01$ & *39:01 & *14:54 & $0.006 \%$ & $-0.008 \%$ & -0.002 & $99.943 \%$ \\
\hline 1270 & ${ }^{\star} 02: 01$ & *39:01 & ${ }^{\star} 15: 02$ & $0.006 \%$ & $-0.037 \%$ & -0.011 & $99.943 \%$ \\
\hline 1270 & ${ }^{\star} 02: 01$ & *39:01 & ${ }^{\star} 16: 02$ & $0.006 \%$ & $0.002 \%$ & 0.002 & $99.943 \%$ \\
\hline 1270 & ${ }^{\star} 02: 01$ & *39:02 & ${ }^{\star} 09: 01$ & $0.006 \%$ & $0.001 \%$ & 0.004 & $99.943 \%$ \\
\hline 1270 & ${ }^{\star} 02: 01$ & *39:04 & *14:05 & $0.006 \%$ & $0.005 \%$ & 0.026 & $99.943 \%$ \\
\hline 1270 & ${ }^{\star} 02: 01$ & *40:01 & ${ }^{\star} 04: 04$ & $0.006 \%$ & $0.004 \%$ & 0.016 & $99.943 \%$ \\
\hline 1270 & ${ }^{\star} 02: 01$ & *40:01 & $\star 10: 01$ & $0.006 \%$ & $0.002 \%$ & 0.004 & $99.944 \%$ \\
\hline 1270 & *02:01 & *40:01 & *12:02 & $0.006 \%$ & $-0.006 \%$ & -0.003 & $99.944 \%$ \\
\hline 1270 & ${ }^{*} 02: 01$ & *40:01 & ${ }^{\star} 14: 03$ & $0.006 \%$ & $-0.004 \%$ & -0.003 & $99.944 \%$ \\
\hline 1270 & ${ }^{\star} 02: 01$ & *40:02 & ${ }^{\star} 01: 01$ & $0.006 \%$ & $-0.049 \%$ & -0.008 & $99.944 \%$ \\
\hline 1270 & ${ }^{\star} 02: 01$ & *40:02 & ${ }^{*} 04: 01$ & $0.006 \%$ & $-0.003 \%$ & -0.003 & $99.944 \%$ \\
\hline 1270 & ${ }^{\star} 02: 01$ & *40:02 & *14:02 & $0.006 \%$ & $0.005 \%$ & 0.083 & $99.944 \%$ \\
\hline 1270 & ${ }^{*} 02: 01$ & *40:02 & *14:06 & $0.006 \%$ & $-0.006 \%$ & -0.005 & $99.944 \%$ \\
\hline 1270 & ${ }^{\star} 02: 01$ & *40:03 & ${ }^{\star} 01: 01$ & $0.006 \%$ & $0.004 \%$ & 0.010 & $99.944 \%$ \\
\hline 1270 & *02:01 & *40:03 & ${ }^{*} 09: 01$ & $0.006 \%$ & $0.000 \%$ & 0.000 & $99.944 \%$ \\
\hline 1270 & *02:01 & *40:03 & *14:06 & $0.006 \%$ & $0.005 \%$ & 0.016 & $99.944 \%$ \\
\hline 1270 & ${ }^{*} 02: 01$ & *40:06 & ${ }^{*} 01: 01$ & $0.006 \%$ & $-0.025 \%$ & -0.006 & $99.945 \%$ \\
\hline
\end{tabular}




\begin{tabular}{|c|c|c|c|c|c|c|c|}
\hline 1270 & ${ }^{\star} 02: 01$ & *40:06 & $\star 04: 05$ & $0.006 \%$ & $-0.066 \%$ & -0.015 & $99.945 \%$ \\
\hline 1270 & ${ }^{\star} 02: 01$ & *40:06 & ${ }^{*} 04: 06$ & $0.006 \%$ & $-0.011 \%$ & -0.003 & $99.945 \%$ \\
\hline 1270 & ${ }^{\star} 02: 01$ & *40:06 & ${ }^{\star} 08: 02$ & $0.006 \%$ & $-0.017 \%$ & -0.004 & $99.945 \%$ \\
\hline 1270 & $\star 02: 01$ & $\star 40: 06$ & ${ }^{\star} 12: 01$ & $0.006 \%$ & $-0.013 \%$ & -0.004 & $99.945 \%$ \\
\hline 1270 & ${ }^{\star} 02: 01$ & *40:06 & ${ }^{\star} 13: 02$ & $0.006 \%$ & $-0.024 \%$ & -0.005 & $99.945 \%$ \\
\hline 1270 & ${ }^{\star} 02: 01$ & *40:06 & *14:05 & $0.006 \%$ & $-0.005 \%$ & -0.002 & $99.945 \%$ \\
\hline 1270 & ${ }^{\star} 02: 01$ & $\star 40: 06$ & *14:06 & $0.006 \%$ & $-0.001 \%$ & -0.001 & $99.945 \%$ \\
\hline 1270 & ${ }^{\star} 02: 01$ & $\star 40: 50$ & ${ }^{\star} 08: 02$ & $0.006 \%$ & $0.006 \%$ & 0.496 & $99.946 \%$ \\
\hline 1270 & ${ }^{\star} 02: 01$ & $\star 40: 50$ & ${ }^{\star} 08: 03$ & $0.006 \%$ & $0.006 \%$ & 0.499 & $99.946 \%$ \\
\hline 1270 & ${ }^{\star} 02: 01$ & $\star 44: 03$ & ${ }^{\star} 08: 03$ & $0.006 \%$ & $-0.057 \%$ & -0.009 & $99.946 \%$ \\
\hline 1270 & ${ }^{\star} 02: 01$ & $\star 44: 03$ & *14:54 & $0.006 \%$ & $-0.019 \%$ & -0.006 & $99.946 \%$ \\
\hline 1270 & ${ }^{\star} 02: 01$ & *46:01 & ${ }^{\star} 04: 05$ & $0.006 \%$ & $-0.071 \%$ & -0.015 & $99.946 \%$ \\
\hline 1270 & ${ }^{\star} 02: 01$ & *46:01 & ${ }^{\star} 08: 02$ & $0.006 \%$ & $-0.019 \%$ & -0.004 & $99.946 \%$ \\
\hline 1270 & ${ }^{\star} 02: 01$ & *46:01 & *12:01 & $0.006 \%$ & $-0.015 \%$ & -0.004 & $99.946 \%$ \\
\hline 1270 & ${ }^{\star} 02: 01$ & *48:01 & ${ }^{\star} 04: 10$ & $0.006 \%$ & $-0.001 \%$ & -0.001 & $99.946 \%$ \\
\hline 1270 & ${ }^{\star} 02: 01$ & *48:01 & ${ }^{\star} 08: 02$ & $0.006 \%$ & $-0.008 \%$ & -0.003 & $99.946 \%$ \\
\hline 1270 & ${ }^{\star} 02: 01$ & *48:01 & *14:07 & $0.006 \%$ & $0.006 \%$ & 0.060 & $99.947 \%$ \\
\hline 1270 & ${ }^{\star} 02: 01$ & *48:01 & *15:02 & $0.006 \%$ & $-0.029 \%$ & -0.010 & $99.947 \%$ \\
\hline 1270 & ${ }^{\star} 02: 01$ & *51:01 & ${ }^{*} 04: 01$ & $0.006 \%$ & $-0.004 \%$ & -0.004 & $99.947 \%$ \\
\hline 1270 & ${ }^{\star} 02: 01$ & *51:01 & ${ }^{*} 04: 04$ & $0.006 \%$ & $0.003 \%$ & 0.011 & $99.947 \%$ \\
\hline 1270 & ${ }^{\star} 02: 01$ & *51:01 & ${ }^{*} 04: 07$ & $0.006 \%$ & $0.000 \%$ & 0.001 & $99.947 \%$ \\
\hline 1270 & ${ }^{\star} 02: 01$ & *51:01 & *10:01 & $0.006 \%$ & $0.000 \%$ & -0.001 & $99.947 \%$ \\
\hline 1270 & ${ }^{\star} 02: 01$ & *51:01 & ${ }^{\star} 14: 06$ & $0.006 \%$ & $-0.008 \%$ & -0.006 & $99.947 \%$ \\
\hline 1270 & ${ }^{\star} 02: 01$ & *51:01 & ${ }^{*} 14: 12$ & $0.006 \%$ & $0.006 \%$ & 0.193 & $99.947 \%$ \\
\hline 1270 & ${ }^{\star} 02: 01$ & $\star 52: 01$ & ${ }^{\star} 04: 06$ & $0.006 \%$ & $-0.036 \%$ & -0.011 & $99.947 \%$ \\
\hline 1270 & ${ }^{\star} 02: 01$ & $\star 52: 01$ & *12:01 & $0.006 \%$ & $-0.042 \%$ & -0.012 & $99.947 \%$ \\
\hline 1270 & ${ }^{\star} 02: 01$ & $\star 52: 01$ & $\star 15: 01$ & $0.006 \%$ & $-0.096 \%$ & -0.013 & $99.947 \%$ \\
\hline 1270 & ${ }^{\star} 02: 01$ & $\star 54: 01$ & ${ }^{\star} 04: 03$ & $0.006 \%$ & $-0.019 \%$ & -0.007 & $99.947 \%$ \\
\hline 1270 & ${ }^{\star} 02: 01$ & $\star 54: 01$ & ${ }^{\star} 04: 10$ & $0.006 \%$ & $-0.013 \%$ & -0.006 & $99.947 \%$ \\
\hline 1270 & ${ }^{\star} 02: 01$ & $\star 54: 01$ & ${ }^{\star} 08: 02$ & $0.006 \%$ & $-0.032 \%$ & -0.008 & $99.948 \%$ \\
\hline 1270 & ${ }^{\star} 02: 01$ & *54:01 & *11:01 & $0.006 \%$ & $-0.019 \%$ & -0.007 & $99.948 \%$ \\
\hline 1270 & ${ }^{\star} 02: 01$ & *54:01 & *13:01 & $0.006 \%$ & $0.001 \%$ & 0.001 & $99.948 \%$ \\
\hline 1270 & ${ }^{*} 02: 01$ & $\star 54: 01$ & $\star 13: 02$ & $0.006 \%$ & $-0.043 \%$ & -0.008 & $99.948 \%$ \\
\hline 1270 & ${ }^{*} 02: 01$ & $\star 54: 01$ & $\star 15: 02$ & $0.006 \%$ & $-0.088 \%$ & -0.012 & $99.948 \%$ \\
\hline 1270 & ${ }^{*} 02: 01$ & $\star 55: 02$ & $\star^{*} 04: 03$ & $0.006 \%$ & $-0.002 \%$ & -0.001 & $99.948 \%$ \\
\hline 1270 & ${ }^{\star} 02: 01$ & $\star 55: 02$ & ${ }^{\star} 08: 02$ & $0.006 \%$ & $-0.007 \%$ & -0.003 & $99.948 \%$ \\
\hline 1270 & ${ }^{\star} 02: 01$ & $\star 55: 02$ & *14:54 & $0.006 \%$ & $-0.004 \%$ & -0.002 & $99.948 \%$ \\
\hline 1270 & ${ }^{\star} 02: 01$ & *55:02 & *14:03 & $0.006 \%$ & $0.001 \%$ & 0.001 & $99.948 \%$ \\
\hline 1270 & ${ }^{\star} 02: 01$ & *55:02 & $\star 15: 02$ & $0.006 \%$ & $-0.025 \%$ & -0.010 & $99.948 \%$ \\
\hline 1270 & ${ }^{\star} 02: 01$ & *55:04 & *13:02 & $0.006 \%$ & $0.005 \%$ & 0.037 & $99.948 \%$ \\
\hline 1270 & ${ }^{\star} 02: 01$ & *55:04 & *14:54 & $0.006 \%$ & $0.005 \%$ & 0.039 & $99.948 \%$ \\
\hline 1270 & ${ }^{\star} 02: 01$ & $\star 55: 04$ & *14:05 & $0.006 \%$ & $0.006 \%$ & 0.042 & $99.949 \%$ \\
\hline 1270 & ${ }^{\star} 02: 01$ & *56:01 & *08:03 & $0.006 \%$ & $-0.003 \%$ & -0.004 & $99.949 \%$ \\
\hline 1270 & ${ }^{\star} 02: 01$ & $\star 56: 01$ & *13:02 & $0.006 \%$ & $0.000 \%$ & 0.000 & $99.949 \%$ \\
\hline 1270 & ${ }^{\star} 02: 01$ & $\star 56: 01$ & *15:01 & $0.006 \%$ & $-0.003 \%$ & -0.003 & $99.949 \%$ \\
\hline 1270 & ${ }^{\star} 02: 01$ & $\star 58: 01$ & ${ }^{\star} 03: 01$ & $0.006 \%$ & $0.006 \%$ & 0.047 & $99.949 \%$ \\
\hline 1270 & ${ }^{\star} 02: 01$ & *58:01 & ${ }^{\star} 13: 02$ & $0.006 \%$ & $0.002 \%$ & 0.003 & $99.949 \%$ \\
\hline 1270 & ${ }^{\star} 02: 01$ & *67:01 & ${ }^{\star} 04: 06$ & $0.006 \%$ & $0.002 \%$ & 0.001 & $99.949 \%$ \\
\hline 1270 & ${ }^{\star} 02: 01$ & *67:01 & ${ }^{\star} 04: 10$ & $0.006 \%$ & $0.003 \%$ & 0.003 & $99.949 \%$ \\
\hline 1270 & *02:01 & *67:01 & *08:03 & $0.006 \%$ & $-0.006 \%$ & -0.005 & $99.949 \%$ \\
\hline 1270 & *02:01 & *67:01 & *09:01 & $0.006 \%$ & $-0.013 \%$ & -0.012 & $99.950 \%$ \\
\hline 1270 & *02:01 & *67:01 & *13:02 & $0.006 \%$ & $-0.002 \%$ & -0.001 & $99.950 \%$ \\
\hline 1270 & ${ }^{\star} 02: 01$ & *67:01 & $\star 15: 02$ & $0.006 \%$ & $-0.009 \%$ & -0.007 & $99.950 \%$ \\
\hline 1270 & ${ }^{*} 02: 03$ & *38:02 & *15:02 & $0.006 \%$ & $0.006 \%$ & 0.144 & $99.950 \%$ \\
\hline
\end{tabular}




\begin{tabular}{|c|c|c|c|c|c|c|c|}
\hline 1270 & ${ }^{*} 02: 03$ & *38:02 & *16:02 & $0.006 \%$ & $0.006 \%$ & 0.144 & $99.951 \%$ \\
\hline 1270 & *02:03 & *51:02 & *14:03 & $0.006 \%$ & $0.006 \%$ & 0.144 & $99.951 \%$ \\
\hline 1270 & ${ }^{*} 02: 03$ & *54:01 & $\star^{*} 04: 05$ & $0.006 \%$ & $0.005 \%$ & 0.135 & $99.951 \%$ \\
\hline 1270 & ${ }^{*} 02: 03$ & *58:01 & *13:02 & $0.006 \%$ & $0.006 \%$ & 0.144 & $99.951 \%$ \\
\hline 1270 & ${ }^{*} 02: 06$ & $\star 07: 02$ & ${ }^{\star} 04: 06$ & $0.006 \%$ & $-0.011 \%$ & -0.003 & $99.951 \%$ \\
\hline 1270 & ${ }^{*} 02: 06$ & *07:02 & ${ }^{\star} 08: 02$ & $0.006 \%$ & $-0.017 \%$ & -0.004 & $99.952 \%$ \\
\hline 1270 & ${ }^{*} 02: 06$ & ${ }^{*} 07: 02$ & ${ }^{*} 08: 03$ & $0.006 \%$ & $-0.040 \%$ & -0.007 & $99.952 \%$ \\
\hline 1270 & ${ }^{*} 02: 06$ & *07:02 & *11:01 & $0.006 \%$ & $-0.009 \%$ & -0.003 & $99.952 \%$ \\
\hline 1270 & ${ }^{*} 02: 06$ & ${ }^{*} 07: 02$ & *15:01 & $0.006 \%$ & $-0.035 \%$ & -0.006 & $99.952 \%$ \\
\hline 1270 & ${ }^{*} 02: 06$ & *13:01 & *11:01 & $0.006 \%$ & $0.003 \%$ & 0.002 & $99.952 \%$ \\
\hline 1270 & ${ }^{*} 02: 06$ & *15:01 & ${ }^{*} 04: 07$ & $0.006 \%$ & $0.002 \%$ & 0.004 & $99.952 \%$ \\
\hline 1270 & ${ }^{*} 02: 06$ & *15:01 & ${ }^{*} 04: 10$ & $0.006 \%$ & $-0.009 \%$ & -0.004 & $99.952 \%$ \\
\hline 1270 & ${ }^{*} 02: 06$ & *15:01 & *12:02 & $0.006 \%$ & $-0.007 \%$ & -0.004 & $99.953 \%$ \\
\hline 1270 & ${ }^{*} 02: 06$ & *15:01 & *13:01 & $0.006 \%$ & $0.002 \%$ & 0.003 & $99.953 \%$ \\
\hline 1270 & ${ }^{*} 02: 06$ & *15:01 & *15:02 & $0.006 \%$ & $-0.071 \%$ & -0.009 & $99.953 \%$ \\
\hline 1270 & ${ }^{*} 02: 06$ & *15:11 & ${ }^{*} 04: 07$ & $0.006 \%$ & $0.005 \%$ & 0.011 & $99.953 \%$ \\
\hline 1270 & ${ }^{*} 02: 06$ & *15:11 & *15:01 & $0.006 \%$ & $-0.001 \%$ & -0.001 & $99.953 \%$ \\
\hline 1270 & ${ }^{*} 02: 06$ & \begin{tabular}{|l|} 
$\pm 15: 18$ \\
$15: 18$
\end{tabular} & *04:10 & $0.006 \%$ & $0.003 \%$ & 0.002 & $99.953 \%$ \\
\hline 1270 & ${ }^{\star} 02: 06$ & *15:18 & ${ }^{\star} 08: 02$ & $0.006 \%$ & $0.000 \%$ & 0.000 & $99.953 \%$ \\
\hline 1270 & ${ }^{\star} 02: 06$ & *15:18 & *14:54 & $0.006 \%$ & $0.001 \%$ & 0.001 & $99.953 \%$ \\
\hline 1270 & ${ }^{\star} 02: 06$ & *15:18 & *14:05 & $0.006 \%$ & $0.003 \%$ & 0.002 & $99.953 \%$ \\
\hline 1270 & ${ }^{*} 02: 06$ & *15:28 & ${ }^{\star} 04: 05$ & $0.006 \%$ & $0.006 \%$ & 1.000 & $99.954 \%$ \\
\hline 1270 & ${ }^{*} 02: 06$ & *27:04 & ${ }^{\star} 08: 03$ & $0.006 \%$ & $0.004 \%$ & 0.021 & $99.954 \%$ \\
\hline 1270 & ${ }^{*} 02: 06$ & *27:04 & ${ }^{\star} 12: 01$ & $0.006 \%$ & $0.005 \%$ & 0.025 & $99.954 \%$ \\
\hline 1270 & ${ }^{*} 02: 06$ & *27:04 & *12:02 & $0.006 \%$ & $0.005 \%$ & 0.027 & $99.954 \%$ \\
\hline 1270 & ${ }^{*} 02: 06$ & *27:04 & *15:02 & $0.006 \%$ & $0.004 \%$ & 0.019 & $99.954 \%$ \\
\hline 1270 & ${ }^{*} 02: 06$ & *27:05 & ${ }^{*} 04: 10$ & $0.006 \%$ & $0.006 \%$ & 0.069 & $99.954 \%$ \\
\hline 1270 & ${ }^{*} 02: 06$ & $\star 35: 01$ & ${ }^{\star} 01: 01$ & $0.006 \%$ & $-0.038 \%$ & -0.007 & $99.954 \%$ \\
\hline 1270 & ${ }^{*} 02: 06$ & *35:01 & *13:01 & $0.006 \%$ & $0.001 \%$ & 0.002 & $99.954 \%$ \\
\hline 1270 & ${ }^{*} 02: 06$ & *35:01 & *16:02 & $0.006 \%$ & $-0.002 \%$ & -0.002 & $99.955 \%$ \\
\hline 1270 & ${ }^{*} 02: 06$ & *38:02 & ${ }^{*} 04: 10$ & $0.006 \%$ & $0.005 \%$ & 0.019 & $99.955 \%$ \\
\hline 1270 & ${ }^{*} 02: 06$ & *38:02 & *08:03 & $0.006 \%$ & $0.004 \%$ & 0.013 & $99.955 \%$ \\
\hline 1270 & *02:06 & *39:01 & *12:01 & $0.006 \%$ & $-0.006 \%$ & -0.002 & $99.955 \%$ \\
\hline 1270 & *02:06 & *39:01 & *12:02 & $0.006 \%$ & $0.000 \%$ & 0.000 & $99.955 \%$ \\
\hline 1270 & *02:06 & *39:01 & *13:02 & $0.006 \%$ & $-0.012 \%$ & -0.003 & $99.955 \%$ \\
\hline 1270 & *02:06 & *39:01 & *15:02 & $0.006 \%$ & $-0.028 \%$ & -0.008 & $99.955 \%$ \\
\hline 1270 & ${ }^{\star} 02: 06$ & *39:02 & *15:01 & $0.006 \%$ & $0.004 \%$ & 0.014 & $99.955 \%$ \\
\hline 1270 & ${ }^{\star} 02: 06$ & *39:04 & ${ }^{\star} 08: 03$ & $0.006 \%$ & $0.004 \%$ & 0.020 & $99.955 \%$ \\
\hline 1270 & ${ }^{\star} 02: 06$ & *40:01 & ${ }^{\star} 08: 02$ & $0.006 \%$ & $-0.016 \%$ & -0.004 & $99.955 \%$ \\
\hline 1270 & ${ }^{\star} 02: 06$ & *40:01 & $\star 14: 06$ & $0.006 \%$ & $-0.001 \%$ & 0.000 & $99.955 \%$ \\
\hline 1270 & ${ }^{\star} 02: 06$ & *40:02 & ${ }^{\star} 04: 03$ & $0.006 \%$ & $-0.015 \%$ & -0.005 & $99.956 \%$ \\
\hline 1270 & ${ }^{*} 02: 06$ & *40:02 & ${ }^{\star} 12: 02$ & $0.006 \%$ & $-0.008 \%$ & -0.004 & $99.956 \%$ \\
\hline 1270 & ${ }^{*} 02: 06$ & *40:02 & ${ }^{\star} 13: 02$ & $0.006 \%$ & $-0.036 \%$ & -0.006 & $99.956 \%$ \\
\hline 1270 & ${ }^{*} 02: 06$ & *40:02 & $\star 14: 03$ & $0.006 \%$ & $-0.006 \%$ & -0.004 & $99.956 \%$ \\
\hline 1270 & ${ }^{*} 02: 06$ & *40:03 & ${ }^{\star} 04: 05$ & $0.006 \%$ & $0.002 \%$ & 0.004 & $99.956 \%$ \\
\hline 1270 & ${ }^{*} 02: 06$ & *40:03 & *15:01 & $0.006 \%$ & $0.003 \%$ & 0.010 & $99.956 \%$ \\
\hline 1270 & ${ }^{*} 02: 06$ & *40:06 & ${ }^{\star} 04: 01$ & $0.006 \%$ & $0.002 \%$ & 0.002 & $99.956 \%$ \\
\hline 1270 & ${ }^{*} 02: 06$ & *40:06 & ${ }^{*} 04: 03$ & $0.006 \%$ & $-0.006 \%$ & -0.002 & $99.956 \%$ \\
\hline 1270 & ${ }^{*} 02: 06$ & *40:06 & *13:02 & $0.006 \%$ & $-0.018 \%$ & -0.004 & $99.956 \%$ \\
\hline 1270 & ${ }^{*} 02: 06$ & *40:06 & *14:06 & $0.006 \%$ & $0.000 \%$ & 0.000 & $99.956 \%$ \\
\hline 1270 & ${ }^{*} 02: 06$ & *40:06 & *14:07 & $0.006 \%$ & $0.005 \%$ & 0.059 & $99.957 \%$ \\
\hline 1270 & ${ }^{*} 02: 06$ & *44:03 & ${ }^{*} 08: 03$ & $0.006 \%$ & $-0.044 \%$ & -0.007 & $99.957 \%$ \\
\hline 1270 & ${ }^{*} 02: 06$ & *44:03 & ${ }^{*} 09: 01$ & $0.006 \%$ & $-0.077 \%$ & -0.013 & $99.957 \%$ \\
\hline 1270 & ${ }^{*} 02: 06$ & *44:03 & *14:03 & $0.006 \%$ & $-0.004 \%$ & -0.002 & $99.957 \%$ \\
\hline
\end{tabular}




\begin{tabular}{|c|c|c|c|c|c|c|c|}
\hline 1270 & ${ }^{\star} 02: 06$ & *46:01 & *15:02 & $0.006 \%$ & $-0.042 \%$ & -0.009 & $99.957 \%$ \\
\hline 1270 & *02:06 & *48:01 & ${ }^{\star} 04: 01$ & $0.006 \%$ & $0.003 \%$ & 0.004 & $99.957 \%$ \\
\hline 1270 & *02:06 & *48:01 & ${ }^{\star} 04: 06$ & $0.006 \%$ & $-0.003 \%$ & -0.001 & $99.957 \%$ \\
\hline 1270 & ${ }^{*} 02: 06$ & *48:01 & ${ }^{\star} 08: 03$ & $0.006 \%$ & $-0.017 \%$ & -0.006 & $99.957 \%$ \\
\hline 1270 & ${ }^{*} 02: 06$ & *48:01 & *11:01 & $0.006 \%$ & $-0.002 \%$ & -0.001 & $99.957 \%$ \\
\hline 1270 & ${ }^{*} 02: 06$ & *48:01 & ${ }^{\star} 12: 01$ & $0.006 \%$ & $-0.004 \%$ & -0.001 & $99.957 \%$ \\
\hline 1270 & ${ }^{*} 02: 06$ & $\star 51: 01$ & ${ }^{*} 04: 06$ & $0.006 \%$ & $-0.022 \%$ & -0.007 & $99.957 \%$ \\
\hline 1270 & ${ }^{*} 02: 06$ & $\star 51: 01$ & ${ }^{*} 04: 07$ & $0.006 \%$ & $0.002 \%$ & 0.003 & $99.957 \%$ \\
\hline 1270 & ${ }^{*} 02: 06$ & $\star 51: 01$ & *12:02 & $0.006 \%$ & $-0.010 \%$ & -0.005 & $99.958 \%$ \\
\hline 1270 & ${ }^{*} 02: 06$ & $\star 51: 01$ & *14:06 & $0.006 \%$ & $-0.005 \%$ & -0.004 & $99.958 \%$ \\
\hline 1270 & ${ }^{*} 02: 06$ & $\star 52: 01$ & ${ }^{*} 09: 01$ & $0.006 \%$ & $-0.145 \%$ & -0.016 & $99.958 \%$ \\
\hline 1270 & ${ }^{*} 02: 06$ & $\star 52: 01$ & *14:54 & $0.006 \%$ & $-0.031 \%$ & -0.009 & $99.958 \%$ \\
\hline 1270 & ${ }^{*} 02: 06$ & $\star 54: 01$ & ${ }^{*} 01: 01$ & $0.006 \%$ & $-0.035 \%$ & -0.006 & $99.958 \%$ \\
\hline 1270 & ${ }^{*} 02: 06$ & $\star 54: 01$ & ${ }^{*} 04: 06$ & $0.006 \%$ & $-0.017 \%$ & -0.005 & $99.958 \%$ \\
\hline 1270 & ${ }^{*} 02: 06$ & $\star 54: 01$ & ${ }^{*} 04: 07$ & $0.006 \%$ & $0.002 \%$ & 0.005 & $99.958 \%$ \\
\hline 1270 & ${ }^{*} 02: 06$ & $\star 54: 01$ & ${ }^{*} 04: 10$ & $0.006 \%$ & $-0.009 \%$ & -0.004 & $99.958 \%$ \\
\hline 1270 & *02:06 & $\star 54: 01$ & ${ }^{*} 09: 01$ & $0.006 \%$ & $-0.095 \%$ & -0.013 & $99.958 \%$ \\
\hline 1270 & ${ }^{*} 02: 06$ & $\star 54: 01$ & *14:05 & $0.006 \%$ & $-0.008 \%$ & -0.004 & $99.958 \%$ \\
\hline 1270 & ${ }^{\star} 02: 06$ & $\star 55: 02$ & ${ }^{\star} 01: 01$ & $0.006 \%$ & $-0.008 \%$ & -0.003 & $99.958 \%$ \\
\hline 1270 & ${ }^{*} 02: 06$ & *55:02 & ${ }^{\star} 12: 02$ & $0.006 \%$ & $0.002 \%$ & 0.001 & $99.958 \%$ \\
\hline 1270 & ${ }^{*} 02: 06$ & *55:02 & ${ }^{\star} 14: 06$ & $0.006 \%$ & $0.003 \%$ & 0.002 & $99.958 \%$ \\
\hline 1270 & ${ }^{\star} 02: 06$ & *55:02 & *14:07 & $0.006 \%$ & $0.006 \%$ & 0.061 & $99.958 \%$ \\
\hline 1270 & ${ }^{*} 02: 06$ & *56:01 & ${ }^{\star} 01: 01$ & $0.006 \%$ & $0.001 \%$ & 0.001 & $99.958 \%$ \\
\hline 1270 & ${ }^{*} 02: 06$ & *56:01 & ${ }^{\star} 08: 03$ & $0.006 \%$ & $-0.002 \%$ & -0.002 & $99.959 \%$ \\
\hline 1270 & ${ }^{*} 02: 06$ & $\star 56: 01$ & *12:02 & $0.006 \%$ & $0.004 \%$ & 0.005 & $99.959 \%$ \\
\hline 1270 & *02:06 & \begin{tabular}{|l|}
$* 56: 03$ \\
\end{tabular} & $\star 12: 01$ & $0.006 \%$ & $0.005 \%$ & 0.022 & $99.959 \%$ \\
\hline 1270 & *02:06 & *58:01 & ${ }^{\star} 04: 05$ & $0.006 \%$ & $-0.002 \%$ & -0.003 & $99.959 \%$ \\
\hline 1270 & ${ }^{*} 02: 06$ & $\star 59: 01$ & *15:01 & $0.006 \%$ & $-0.008 \%$ & -0.004 & $99.959 \%$ \\
\hline 1270 & *02:06 & *67:01 & ${ }^{\star} 04: 05$ & $0.006 \%$ & $-0.009 \%$ & -0.008 & $99.959 \%$ \\
\hline 1270 & ${ }^{*} 02: 07$ & *15:01 & *14:54 & $0.006 \%$ & $-0.004 \%$ & -0.001 & $99.959 \%$ \\
\hline 1270 & ${ }^{*} 02: 07$ & *15:07 & *14:03 & $0.006 \%$ & $0.005 \%$ & 0.009 & $99.959 \%$ \\
\hline 1270 & ${ }^{*} 02: 07$ & *35:01 & ${ }^{*} 08: 03$ & $0.006 \%$ & $-0.019 \%$ & -0.005 & $99.959 \%$ \\
\hline 1270 & *02:07 & *35:01 & *12:01 & $0.006 \%$ & $-0.005 \%$ & -0.001 & $99.960 \%$ \\
\hline 1270 & ${ }^{*} 02: 07$ & *35:01 & *14:54 & $0.006 \%$ & $-0.004 \%$ & -0.001 & $99.960 \%$ \\
\hline 1270 & ${ }^{*} 02: 07$ & *35:01 & *14:02 & $0.006 \%$ & $0.006 \%$ & 0.088 & $99.960 \%$ \\
\hline 1270 & ${ }^{*} 02: 07$ & *35:01 & *14:03 & $0.006 \%$ & $0.001 \%$ & 0.001 & $99.960 \%$ \\
\hline 1270 & ${ }^{*} 02: 07$ & *37:01 & *10:01 & $0.006 \%$ & $0.006 \%$ & 0.010 & $99.960 \%$ \\
\hline 1270 & ${ }^{\star} 02: 07$ & *39:01 & ${ }^{\star} 08: 02$ & $0.006 \%$ & $0.001 \%$ & 0.000 & $99.960 \%$ \\
\hline 1270 & ${ }^{\star} 02: 07$ & *39:01 & *14:06 & $0.006 \%$ & $0.004 \%$ & 0.003 & $99.961 \%$ \\
\hline 1270 & ${ }^{\star} 02: 07$ & *39:02 & ${ }^{\star} 09: 01$ & $0.006 \%$ & $0.004 \%$ & 0.016 & $99.961 \%$ \\
\hline 1270 & ${ }^{\star} 02: 07$ & *40:01 & *14:54 & $0.006 \%$ & $-0.001 \%$ & 0.000 & $99.961 \%$ \\
\hline 1270 & ${ }^{*} 02: 07$ & *40:01 & *14:05 & $0.006 \%$ & $0.002 \%$ & 0.001 & $99.961 \%$ \\
\hline 1270 & ${ }^{*} 02: 07$ & *40:02 & ${ }^{\star} 01: 01$ & $0.006 \%$ & $-0.011 \%$ & -0.003 & $99.961 \%$ \\
\hline 1270 & ${ }^{*} 02: 07$ & *40:02 & ${ }^{\star} 08: 02$ & $0.006 \%$ & $-0.006 \%$ & -0.002 & $99.961 \%$ \\
\hline 1270 & ${ }^{*} 02: 07$ & *40:02 & ${ }^{\star} 09: 01$ & $0.006 \%$ & $-0.035 \%$ & -0.010 & $99.962 \%$ \\
\hline 1270 & ${ }^{*} 02: 07$ & *40:02 & *11:01 & $0.006 \%$ & $-0.002 \%$ & -0.001 & $99.962 \%$ \\
\hline 1270 & ${ }^{*} 02: 07$ & *40:02 & ${ }^{\star} 14: 54$ & $0.006 \%$ & $-0.004 \%$ & -0.001 & $99.962 \%$ \\
\hline 1270 & ${ }^{*} 02: 07$ & *40:02 & *15:01 & $0.006 \%$ & $-0.016 \%$ & -0.005 & $99.962 \%$ \\
\hline 1270 & ${ }^{*} 02: 07$ & *40:06 & ${ }^{*} 04: 05$ & $0.006 \%$ & $-0.016 \%$ & -0.005 & $99.962 \%$ \\
\hline 1270 & ${ }^{*} 02: 07$ & *40:06 & ${ }^{*} 08: 02$ & $0.006 \%$ & $-0.001 \%$ & 0.000 & $99.962 \%$ \\
\hline 1270 & ${ }^{*} 02: 07$ & *40:06 & *12:01 & $0.006 \%$ & $0.000 \%$ & 0.000 & $99.962 \%$ \\
\hline 1270 & *02:07 & *44:03 & *08:03 & $0.006 \%$ & $-0.013 \%$ & -0.004 & $99.962 \%$ \\
\hline 1270 & ${ }^{*} 02: 07$ & *44:03 & ${ }^{*} 09: 01$ & $0.006 \%$ & $-0.025 \%$ & -0.007 & $99.963 \%$ \\
\hline 1270 & *02:07 & *44:03 & *14:03 & $0.006 \%$ & $0.002 \%$ & 0.001 & $99.963 \%$ \\
\hline
\end{tabular}




\begin{tabular}{|c|c|c|c|c|c|c|c|}
\hline 1270 & ${ }^{\star} 02: 07$ & $\star 46: 01$ & ${ }^{\star} 04: 03$ & $0.006 \%$ & $0.001 \%$ & 0.000 & $99.963 \%$ \\
\hline 1270 & *02:07 & *46:01 & ${ }^{*} 08: 02$ & $0.006 \%$ & $-0.002 \%$ & 0.000 & $99.963 \%$ \\
\hline 1270 & *02:07 & $\star 46: 01$ & *14:54 & $0.006 \%$ & $0.000 \%$ & 0.000 & $99.963 \%$ \\
\hline 1270 & $\star 02: 07$ & $\star 46: 01$ & *14:03 & $0.006 \%$ & $0.003 \%$ & 0.002 & $99.963 \%$ \\
\hline 1270 & ${ }^{\star} 02: 07$ & $\star 46: 01$ & *14:07 & $0.006 \%$ & $0.006 \%$ & 0.061 & $99.963 \%$ \\
\hline 1270 & ${ }^{\star} 02: 07$ & $\star 46: 01$ & *15:02 & $0.006 \%$ & $-0.012 \%$ & -0.004 & $99.963 \%$ \\
\hline 1270 & ${ }^{\star} 02: 07$ & *48:01 & *14:05 & $0.006 \%$ & $0.004 \%$ & 0.002 & $99.963 \%$ \\
\hline 1270 & ${ }^{\star} 02: 07$ & $\star 51: 01$ & ${ }^{\star} 04: 03$ & $0.006 \%$ & $-0.003 \%$ & -0.001 & $99.964 \%$ \\
\hline 1270 & ${ }^{\star} 02: 07$ & $\star 51: 01$ & ${ }^{\star} 04: 06$ & $0.006 \%$ & $-0.005 \%$ & -0.001 & $99.964 \%$ \\
\hline 1270 & ${ }^{\star} 02: 07$ & $\star 51: 01$ & ${ }^{\star} 08: 03$ & $0.006 \%$ & $-0.022 \%$ & -0.006 & $99.964 \%$ \\
\hline 1270 & ${ }^{\star} 02: 07$ & $\star 51: 01$ & $\star 14: 05$ & $0.006 \%$ & $-0.001 \%$ & 0.000 & $99.964 \%$ \\
\hline 1270 & ${ }^{\star} 02: 07$ & $\star 51: 01$ & $\star 16: 02$ & $0.006 \%$ & $0.003 \%$ & 0.003 & $99.964 \%$ \\
\hline 1270 & ${ }^{\star} 02: 07$ & $\star 52: 01$ & ${ }^{\star} 04: 05$ & $0.006 \%$ & $-0.048 \%$ & -0.014 & $99.964 \%$ \\
\hline 1270 & ${ }^{\star} 02: 07$ & $\star 52: 01$ & *15:01 & $0.006 \%$ & $-0.025 \%$ & -0.007 & $99.964 \%$ \\
\hline 1270 & ${ }^{\star} 02: 07$ & $\star 54: 01$ & ${ }^{\star} 04: 06$ & $0.006 \%$ & $-0.003 \%$ & -0.001 & $99.964 \%$ \\
\hline 1270 & ${ }^{\star} 02: 07$ & $\star 55: 02$ & ${ }^{\star} 01: 01$ & $0.006 \%$ & $0.001 \%$ & 0.000 & $99.964 \%$ \\
\hline 1270 & ${ }^{\star} 02: 07$ & $\star 55: 02$ & ${ }^{\star} 04: 06$ & $0.006 \%$ & $0.003 \%$ & 0.001 & $99.965 \%$ \\
\hline 1270 & ${ }^{\star} 02: 07$ & *55:02 & ${ }^{\star} 09: 01$ & $0.006 \%$ & $-0.007 \%$ & -0.003 & $99.965 \%$ \\
\hline 1270 & ${ }^{\star} 02: 07$ & $\star 56: 01$ & ${ }^{*} 04: 10$ & $0.006 \%$ & $0.005 \%$ & 0.006 & $99.965 \%$ \\
\hline 1270 & ${ }^{\star} 02: 07$ & $\star 56: 01$ & *14:05 & $0.006 \%$ & $0.005 \%$ & 0.006 & $99.965 \%$ \\
\hline 1270 & ${ }^{\star} 02: 07$ & $\star 58: 01$ & *15:01 & $0.006 \%$ & $0.004 \%$ & 0.007 & $99.965 \%$ \\
\hline 1270 & ${ }^{\star} 02: 07$ & $\star 59: 01$ & ${ }^{*} 04: 05$ & $0.006 \%$ & $-0.003 \%$ & -0.002 & $99.965 \%$ \\
\hline 1270 & ${ }^{\star} 02: 10$ & *15:01 & *14:29 & $0.006 \%$ & $0.006 \%$ & 0.251 & $99.965 \%$ \\
\hline 1270 & ${ }^{\star} 02: 10$ & *35:01 & ${ }^{\star} 04: 03$ & $0.006 \%$ & $0.005 \%$ & 0.012 & $99.966 \%$ \\
\hline 1270 & ${ }^{\star} 02: 10$ & $\star 35: 01$ & *14:54 & $0.006 \%$ & $0.005 \%$ & 0.011 & $99.966 \%$ \\
\hline 1270 & ${ }^{\star} 02: 10$ & $\star 40: 01$ & ${ }^{\star} 09: 01$ & $0.006 \%$ & $0.003 \%$ & 0.006 & $99.966 \%$ \\
\hline 1270 & ${ }^{\star} 02: 10$ & $\star 40: 01$ & $\star 11: 01$ & $0.006 \%$ & $0.005 \%$ & 0.013 & $99.966 \%$ \\
\hline 1270 & ${ }^{\star} 02: 10$ & $\star 40: 06$ & ${ }^{\star} 04: 06$ & $0.006 \%$ & $0.005 \%$ & 0.013 & $99.966 \%$ \\
\hline 1270 & ${ }^{\star} 02: 10$ & $\star 40: 06$ & ${ }^{\star} 15: 01$ & $0.006 \%$ & $0.004 \%$ & 0.011 & $99.966 \%$ \\
\hline 1270 & ${ }^{\star} 02: 10$ & $\star 40: 06$ & ${ }^{\star} 16: 02$ & $0.006 \%$ & $0.006 \%$ & 0.014 & $99.966 \%$ \\
\hline 1270 & ${ }^{\star} 02: 10$ & $\star 51: 01$ & *14:54 & $0.006 \%$ & $0.004 \%$ & 0.011 & $99.966 \%$ \\
\hline 1270 & ${ }^{\star} 02: 10$ & $\star 54: 01$ & ${ }^{\star} 04: 05$ & $0.006 \%$ & $0.002 \%$ & 0.004 & $99.966 \%$ \\
\hline 1270 & ${ }^{\star} 02: 13$ & $\star 40: 02$ & *14:05 & $0.006 \%$ & $0.006 \%$ & 1.006 & $99.967 \%$ \\
\hline 1270 & ${ }^{\star} 02: 18$ & $\star 38: 02$ & ${ }^{\star} 04: 10$ & $0.006 \%$ & $0.006 \%$ & 0.126 & $99.967 \%$ \\
\hline 1270 & ${ }^{\star} 02: 28$ & $\star 55: 02$ & ${ }^{\star} 08: 03$ & $0.006 \%$ & $0.006 \%$ & 1.007 & $99.967 \%$ \\
\hline 1270 & ${ }^{\star} 03: 01$ & $\star 07: 02$ & ${ }^{\star} 07: 01$ & $0.006 \%$ & $0.006 \%$ & 0.014 & $99.967 \%$ \\
\hline 1270 & ${ }^{*} 03: 01$ & *15:01 & *15:01 & $0.006 \%$ & $0.003 \%$ & 0.008 & $99.967 \%$ \\
\hline 1270 & ${ }^{*} 03: 01$ & *35:01 & *12:01 & $0.006 \%$ & $0.005 \%$ & 0.011 & $99.968 \%$ \\
\hline 1270 & ${ }^{*} 03: 01$ & *35:01 & $\star 15: 01$ & $0.006 \%$ & $0.003 \%$ & 0.008 & $99.968 \%$ \\
\hline 1270 & ${ }^{*} 03: 01$ & *40:02 & *14:05 & $0.006 \%$ & $0.005 \%$ & 0.013 & $99.968 \%$ \\
\hline 1270 & ${ }^{\star} 03: 01$ & *44:02 & ${ }^{*} 04: 10$ & $0.006 \%$ & $0.006 \%$ & 0.015 & $99.968 \%$ \\
\hline 1270 & *03:01 & $* 44: 02$ & ${ }^{*} 08: 03$ & $0.006 \%$ & $0.006 \%$ & 0.015 & $99.968 \%$ \\
\hline 1270 & *03:01 & $* 44: 02$ & *12:01 & $0.006 \%$ & $0.006 \%$ & 0.015 & $99.968 \%$ \\
\hline 1270 & ${ }^{\star} 03: 01$ & $\star 50: 01$ & ${ }^{\star} 07: 01$ & $0.006 \%$ & $0.006 \%$ & 0.502 & $99.969 \%$ \\
\hline 1270 & ${ }^{\star} 03: 01$ & $\star 51: 01$ & *15:01 & $0.006 \%$ & $0.003 \%$ & 0.007 & $99.969 \%$ \\
\hline 1270 & *03:01 & $\star 52: 01$ & *12:01 & $0.006 \%$ & $0.004 \%$ & 0.010 & $99.969 \%$ \\
\hline 1270 & ${ }^{\star} 03: 01$ & $\star 56: 01$ & ${ }^{\star} 09: 01$ & $0.006 \%$ & $0.005 \%$ & 0.013 & $99.969 \%$ \\
\hline 1270 & ${ }^{\star} 03: 01$ & *67:01 & *15:01 & $0.006 \%$ & $0.005 \%$ & 0.013 & $99.969 \%$ \\
\hline 1270 & ${ }^{\star} 03: 02$ & $\star 13: 02$ & ${ }^{\star} 01: 01$ & $0.006 \%$ & $0.006 \%$ & 0.059 & $99.970 \%$ \\
\hline 1270 & ${ }^{\star} 03: 02$ & $\star 13: 02$ & *14:54 & $0.006 \%$ & $0.006 \%$ & 0.059 & $99.970 \%$ \\
\hline 1270 & *11:01 & ${ }^{*} 07: 02$ & *08:03 & $0.006 \%$ & $-0.038 \%$ & -0.007 & $99.970 \%$ \\
\hline 1270 & *11:01 & ${ }^{\star} 07: 05$ & *12:02 & $0.006 \%$ & $0.006 \%$ & 0.124 & $99.970 \%$ \\
\hline 1270 & *11:01 & $\star 13: 01$ & ${ }^{\star} 09: 01$ & $0.006 \%$ & $-0.010 \%$ & -0.008 & $99.970 \%$ \\
\hline 1270 & *11:01 & $\star 13: 01$ & *15:01 & $0.006 \%$ & $-0.003 \%$ & -0.002 & $99.970 \%$ \\
\hline
\end{tabular}




\begin{tabular}{|c|c|c|c|c|c|c|c|}
\hline 1270 & *11:01 & *13:01 & *15:11 & $0.006 \%$ & $0.006 \%$ & 1.006 & $99.971 \%$ \\
\hline 1270 & *11:01 & $\star 15: 01$ & ${ }^{\star} 01: 01$ & $0.006 \%$ & $-0.035 \%$ & -0.006 & $99.971 \%$ \\
\hline 1270 & *11:01 & $\star 15: 01$ & *13:07 & $0.006 \%$ & $0.006 \%$ & 0.497 & $99.971 \%$ \\
\hline 1270 & *11:01 & *15:02 & ${ }^{\star} 09: 01$ & $0.006 \%$ & $0.005 \%$ & 0.114 & $99.971 \%$ \\
\hline 1270 & *11:01 & *15:11 & ${ }^{\star} 08: 03$ & $0.006 \%$ & $-0.002 \%$ & -0.002 & $99.971 \%$ \\
\hline 1270 & *11:01 & *15:11 & ${ }^{\star} 15: 02$ & $0.006 \%$ & $-0.004 \%$ & -0.004 & $99.971 \%$ \\
\hline 1270 & *11:01 & *15:18 & ${ }^{*} 04: 05$ & $0.006 \%$ & $-0.012 \%$ & -0.008 & $99.971 \%$ \\
\hline 1270 & *11:01 & *15:18 & ${ }^{*} 08: 03$ & $0.006 \%$ & $-0.005 \%$ & -0.004 & $99.971 \%$ \\
\hline 1270 & *11:01 & *15:18 & *12:01 & $0.006 \%$ & $0.001 \%$ & 0.001 & $99.971 \%$ \\
\hline 1270 & *11:01 & *15:18 & *15:02 & $0.006 \%$ & $-0.008 \%$ & -0.006 & $99.972 \%$ \\
\hline 1270 & *11:01 & *15:35 & *15:02 & $0.006 \%$ & $0.006 \%$ & 0.499 & $99.972 \%$ \\
\hline 1270 & *11:01 & *27:04 & *12:01 & $0.006 \%$ & $0.005 \%$ & 0.025 & $99.972 \%$ \\
\hline 1270 & *11:01 & *27:05 & ${ }^{*} 04: 03$ & $0.006 \%$ & $0.006 \%$ & 0.069 & $99.972 \%$ \\
\hline 1270 & *11:01 & *35:01 & ${ }^{*} 04: 07$ & $0.006 \%$ & $0.002 \%$ & 0.004 & $99.972 \%$ \\
\hline 1270 & *11:01 & *35:01 & *13:02 & $0.006 \%$ & $-0.035 \%$ & -0.006 & $99.972 \%$ \\
\hline 1270 & *11:01 & *35:01 & *14:06 & $0.006 \%$ & $-0.004 \%$ & -0.003 & $99.972 \%$ \\
\hline 1270 & *11:01 & $\star 38: 02$ & ${ }^{*} 01: 01$ & $0.006 \%$ & $0.004 \%$ & 0.016 & $99.972 \%$ \\
\hline 1270 & *11:01 & $\star 38: 02$ & *15:02 & $0.006 \%$ & $0.003 \%$ & 0.011 & $99.973 \%$ \\
\hline 1270 & *11:01 & *39:01 & ${ }^{\star} 01: 01$ & $0.006 \%$ & $-0.012 \%$ & -0.004 & $99.973 \%$ \\
\hline 1270 & *11:01 & *39:01 & ${ }^{\star} 04: 10$ & $0.006 \%$ & $-0.001 \%$ & 0.000 & $99.973 \%$ \\
\hline 1270 & *11:01 & *39:01 & ${ }^{\star} 08: 02$ & $0.006 \%$ & $-0.007 \%$ & -0.002 & $99.973 \%$ \\
\hline 1270 & *11:01 & *39:01 & ${ }^{\star} 12: 01$ & $0.006 \%$ & $-0.005 \%$ & -0.002 & $99.973 \%$ \\
\hline 1270 & *11:01 & *39:01 & ${ }^{\star} 14: 54$ & $0.006 \%$ & $-0.005 \%$ & -0.001 & $99.973 \%$ \\
\hline 1270 & *11:01 & *39:01 & ${ }^{\star} 14: 03$ & $0.006 \%$ & $0.001 \%$ & 0.001 & $99.973 \%$ \\
\hline 1270 & *11:01 & *39:01 & *15:02 & $0.006 \%$ & $-0.027 \%$ & -0.008 & $99.973 \%$ \\
\hline 1270 & *11:01 & *39:02 & ${ }^{*} 04: 05$ & $0.006 \%$ & $0.002 \%$ & 0.009 & $99.973 \%$ \\
\hline 1270 & *11:01 & *39:02 & ${ }^{\star} 09: 01$ & $0.006 \%$ & $0.002 \%$ & 0.008 & $99.973 \%$ \\
\hline 1270 & *11:01 & *40:01 & ${ }^{*} 04: 06$ & $0.006 \%$ & $-0.010 \%$ & -0.003 & $99.974 \%$ \\
\hline 1270 & *11:01 & *40:01 & ${ }^{\star} 08: 02$ & $0.006 \%$ & $-0.015 \%$ & -0.003 & $99.974 \%$ \\
\hline 1270 & *11:01 & *40:01 & ${ }^{*} 08: 09$ & $0.006 \%$ & $0.006 \%$ & 0.122 & $99.974 \%$ \\
\hline 1270 & *11:01 & *40:01 & *14:03 & $0.006 \%$ & $-0.002 \%$ & -0.001 & $99.974 \%$ \\
\hline 1270 & *11:01 & *40:02 & ${ }^{*} 04: 03$ & $0.006 \%$ & $-0.014 \%$ & -0.005 & $99.974 \%$ \\
\hline 1270 & *11:01 & *40:02 & ${ }^{*} 04: 06$ & $0.006 \%$ & $-0.017 \%$ & -0.005 & $99.974 \%$ \\
\hline 1270 & *11:01 & *40:02 & *12:01 & $0.006 \%$ & $-0.020 \%$ & -0.006 & $99.974 \%$ \\
\hline 1270 & *11:01 & $* 40: 03$ & *14:05 & $0.006 \%$ & $0.005 \%$ & 0.015 & $99.974 \%$ \\
\hline 1270 & *11:01 & $* 40: 06$ & ${ }^{*} 04: 10$ & $0.006 \%$ & $-0.003 \%$ & -0.001 & $99.974 \%$ \\
\hline 1270 & *11:01 & $\star 40: 06$ & *14:54 & $0.006 \%$ & $-0.008 \%$ & -0.002 & $99.974 \%$ \\
\hline 1270 & *11:01 & $\star 40: 06$ & $\star 14: 06$ & $0.006 \%$ & $0.001 \%$ & 0.000 & $99.974 \%$ \\
\hline 1270 & *11:01 & $\star 40: 06$ & $\star 15: 02$ & $0.006 \%$ & $-0.038 \%$ & -0.008 & $99.974 \%$ \\
\hline 1270 & *11:01 & $\star 44: 03$ & ${ }^{\star} 08: 03$ & $0.006 \%$ & $-0.043 \%$ & -0.007 & $99.975 \%$ \\
\hline 1270 & *11:01 & *46:01 & ${ }^{\star} 04: 06$ & $0.006 \%$ & $-0.008 \%$ & -0.003 & $99.975 \%$ \\
\hline 1270 & *11:01 & *46:01 & $\star 15: 02$ & $0.006 \%$ & $-0.041 \%$ & -0.008 & $99.975 \%$ \\
\hline 1270 & *11:01 & *48:01 & ${ }^{\star} 04: 10$ & $0.006 \%$ & $0.000 \%$ & 0.000 & $99.975 \%$ \\
\hline 1270 & *11:01 & *48:01 & ${ }^{\star} 08: 02$ & $0.006 \%$ & $-0.005 \%$ & -0.002 & $99.975 \%$ \\
\hline 1270 & *11:01 & *48:01 & *11:01 & $0.006 \%$ & $-0.001 \%$ & 0.000 & $99.975 \%$ \\
\hline 1270 & *11:01 & *48:01 & ${ }^{\star} 12: 02$ & $0.006 \%$ & $0.001 \%$ & 0.001 & $99.975 \%$ \\
\hline 1270 & *11:01 & *48:01 & ${ }^{\star} 14: 54$ & $0.006 \%$ & $-0.003 \%$ & -0.001 & $99.975 \%$ \\
\hline 1270 & *11:01 & *48:01 & *14:03 & $0.006 \%$ & $0.002 \%$ & 0.001 & $99.975 \%$ \\
\hline 1270 & *11:01 & *48:01 & *14:12 & $0.006 \%$ & $0.006 \%$ & 0.198 & $99.975 \%$ \\
\hline 1270 & *11:01 & *51:01 & ${ }^{*} 01: 01$ & $0.006 \%$ & $-0.043 \%$ & -0.007 & $99.975 \%$ \\
\hline 1270 & *11:01 & $\star 51: 01$ & ${ }^{*} 04: 03$ & $0.006 \%$ & $-0.018 \%$ & -0.006 & $99.975 \%$ \\
\hline 1270 & *11:01 & $\star 51: 01$ & *12:02 & $0.006 \%$ & $-0.009 \%$ & -0.005 & $99.975 \%$ \\
\hline 1270 & *11:01 & $\star 51: 01$ & *13:02 & $0.006 \%$ & $-0.040 \%$ & -0.007 & $99.975 \%$ \\
\hline 1270 & *11:01 & $\star 51: 02$ & ${ }^{\star} 09: 01$ & $0.006 \%$ & $0.003 \%$ & 0.017 & $99.975 \%$ \\
\hline
\end{tabular}




\begin{tabular}{|c|c|c|c|c|c|c|c|}
\hline 1270 & *11:01 & $\star 52: 01$ & ${ }^{\star} 01: 01$ & $0.006 \%$ & $-0.053 \%$ & -0.009 & $99.975 \%$ \\
\hline 1270 & *11:01 & $\star 52: 01$ & *15:01 & $0.006 \%$ & $-0.073 \%$ & -0.010 & $99.976 \%$ \\
\hline 1270 & *11:01 & $\star 54: 01$ & ${ }^{\star} 01: 01$ & $0.006 \%$ & $-0.034 \%$ & -0.006 & $99.976 \%$ \\
\hline 1270 & *11:01 & $\star 54: 01$ & ${ }^{\star} 04: 01$ & $0.006 \%$ & $0.000 \%$ & 0.000 & $99.976 \%$ \\
\hline 1270 & *11:01 & $\star 54: 01$ & ${ }^{\star} 08: 09$ & $0.006 \%$ & $0.006 \%$ & 0.120 & $99.976 \%$ \\
\hline 1270 & *11:01 & $\star 54: 01$ & ${ }^{\star} 12: 02$ & $0.006 \%$ & $-0.007 \%$ & -0.004 & $99.976 \%$ \\
\hline 1270 & *11:01 & $\star 54: 01$ & *14:03 & $0.006 \%$ & $-0.005 \%$ & -0.003 & $99.976 \%$ \\
\hline 1270 & *11:01 & $\star 54: 01$ & *16:02 & $0.006 \%$ & $-0.001 \%$ & -0.001 & $99.976 \%$ \\
\hline 1270 & *11:01 & $\star 55: 02$ & ${ }^{\star} 04: 01$ & $0.006 \%$ & $0.004 \%$ & 0.004 & $99.976 \%$ \\
\hline 1270 & *11:01 & $\star 55: 02$ & ${ }^{\star} 04: 06$ & $0.006 \%$ & $-0.001 \%$ & -0.001 & $99.976 \%$ \\
\hline 1270 & *11:01 & $\star 55: 02$ & ${ }^{\star} 13: 02$ & $0.006 \%$ & $-0.007 \%$ & -0.003 & $99.976 \%$ \\
\hline 1270 & *11:01 & $\star 55: 02$ & *14:05 & $0.006 \%$ & $0.001 \%$ & 0.001 & $99.976 \%$ \\
\hline 1270 & *11:01 & $\star 56: 01$ & *15:02 & $0.006 \%$ & $-0.003 \%$ & -0.003 & $99.976 \%$ \\
\hline 1270 & *11:01 & $\star 56: 03$ & ${ }^{\star} 04: 05$ & $0.006 \%$ & $0.003 \%$ & 0.013 & $99.976 \%$ \\
\hline 1270 & *11:01 & $\star 56: 03$ & ${ }^{\star} 08: 03$ & $0.006 \%$ & $0.004 \%$ & 0.018 & $99.976 \%$ \\
\hline 1270 & *11:01 & *67:01 & ${ }^{\star} 01: 01$ & $0.006 \%$ & $0.000 \%$ & 0.000 & $99.976 \%$ \\
\hline 1270 & *11:01 & *67:01 & ${ }^{\star} 08: 02$ & $0.006 \%$ & $0.001 \%$ & 0.001 & $99.976 \%$ \\
\hline 1270 & *11:01 & *67:01 & ${ }^{\star} 08: 03$ & $0.006 \%$ & $-0.003 \%$ & -0.003 & $99.976 \%$ \\
\hline 1270 & *11:01 & *67:01 & *11:01 & $0.006 \%$ & $0.003 \%$ & 0.003 & $99.977 \%$ \\
\hline 1270 & *11:01 & *67:01 & *12:01 & $0.006 \%$ & $0.002 \%$ & 0.002 & $99.977 \%$ \\
\hline 1270 & *11:01 & *67:01 & *13:02 & $0.006 \%$ & $0.000 \%$ & 0.000 & $99.977 \%$ \\
\hline 1270 & *11:01 & *67:01 & *15:02 & $0.006 \%$ & $-0.005 \%$ & -0.005 & $99.977 \%$ \\
\hline 1270 & *11:02 & *15:11 & ${ }^{*} 09: 01$ & $0.006 \%$ & $0.006 \%$ & 0.033 & $99.977 \%$ \\
\hline 1270 & *11:02 & *27:04 & ${ }^{*} 09: 01$ & $0.006 \%$ & $0.006 \%$ & 0.034 & $99.977 \%$ \\
\hline 1270 & *11:02 & $\star 27: 04$ & *12:01 & $0.006 \%$ & $0.006 \%$ & 0.035 & $99.977 \%$ \\
\hline 1270 & *11:02 & $\star 27: 04$ & *14:54 & $0.006 \%$ & $0.006 \%$ & 0.035 & $99.977 \%$ \\
\hline 1270 & *11:02 & $\star 27: 04$ & *14:05 & $0.006 \%$ & $0.006 \%$ & 0.035 & $99.978 \%$ \\
\hline 1270 & $\star 11: 02$ & $\star 27: 04$ & ${ }^{\star} 14: 06$ & $0.006 \%$ & $0.006 \%$ & 0.035 & $99.978 \%$ \\
\hline 1270 & *11:02 & *35:01 & ${ }^{\star} 04: 03$ & $0.006 \%$ & $0.005 \%$ & 0.032 & $99.978 \%$ \\
\hline 1270 & $\star 11: 02$ & $\star 40: 01$ & ${ }^{\star} 04: 05$ & $0.006 \%$ & $0.005 \%$ & 0.028 & $99.978 \%$ \\
\hline 1270 & *11:02 & $\star 40: 01$ & *11:01 & $0.006 \%$ & $0.006 \%$ & 0.033 & $99.978 \%$ \\
\hline 1270 & *11:02 & *40:02 & $\star^{*} 09: 01$ & $0.006 \%$ & $0.004 \%$ & 0.023 & $99.978 \%$ \\
\hline 1270 & *11:02 & $\star 51: 01$ & *14:05 & $0.006 \%$ & $0.006 \%$ & 0.033 & $99.978 \%$ \\
\hline 1270 & $\star 11: 02$ & $\star 54: 01$ & $\star 04: 05$ & $0.006 \%$ & $0.004 \%$ & 0.025 & $99.978 \%$ \\
\hline 1270 & *11:02 & $\star 55: 02$ & $\star 15: 01$ & $0.006 \%$ & $0.006 \%$ & 0.033 & $99.978 \%$ \\
\hline 1270 & *11:05 & *67:01 & *15:01 & $0.006 \%$ & $0.006 \%$ & 1.006 & $99.978 \%$ \\
\hline 1270 & *11:43 & *67:01 & *15:01 & $0.006 \%$ & $0.006 \%$ & 1.006 & $99.979 \%$ \\
\hline 1270 & *24:02 & $\star 07: 02$ & *13:02 & $0.006 \%$ & $-0.107 \%$ & -0.020 & $99.979 \%$ \\
\hline 1270 & *24:02 & $\star 07: 02$ & $\star 14: 06$ & $0.006 \%$ & $-0.020 \%$ & -0.016 & $99.979 \%$ \\
\hline 1270 & *24:02 & ${ }^{*} 07: 02$ & $\star 16: 02$ & $0.006 \%$ & $-0.014 \%$ & -0.015 & $99.979 \%$ \\
\hline 1270 & *24:02 & $\star 07: 05$ & *11:06 & $0.006 \%$ & $0.006 \%$ & 0.251 & $99.979 \%$ \\
\hline 1270 & *24:02 & $\star 07: 31$ & ${ }^{*} 04: 06$ & $0.006 \%$ & $0.006 \%$ & 0.999 & $99.979 \%$ \\
\hline 1270 & *24:02 & *13:01 & *04:05 & $0.006 \%$ & $-0.054 \%$ & -0.047 & $99.979 \%$ \\
\hline 1270 & *24:02 & *13:01 & ${ }^{\star} 08: 03$ & $0.006 \%$ & $-0.032 \%$ & -0.027 & $99.979 \%$ \\
\hline 1270 & *24:02 & *13:01 & *12:01 & $0.006 \%$ & $-0.010 \%$ & -0.008 & $99.979 \%$ \\
\hline 1270 & *24:02 & *13:01 & *16:02 & $0.006 \%$ & $0.002 \%$ & 0.002 & $99.979 \%$ \\
\hline 1270 & *24:02 & *13:02 & ${ }^{\star} 07: 01$ & $0.006 \%$ & $0.005 \%$ & 0.017 & $99.980 \%$ \\
\hline 1270 & $\star 24: 02$ & $\star 15: 01$ & ${ }^{\star} 04: 07$ & $0.006 \%$ & $-0.008 \%$ & -0.017 & $99.980 \%$ \\
\hline 1270 & $\star 24: 02$ & $\star 15: 01$ & *15:02 & $0.006 \%$ & $-0.293 \%$ & -0.039 & $99.980 \%$ \\
\hline 1270 & *24:02 & *15:01 & *16:02 & $0.006 \%$ & $-0.022 \%$ & -0.023 & $99.980 \%$ \\
\hline 1270 & *24:02 & *15:02 & *12:02 & $0.006 \%$ & $0.006 \%$ & 0.120 & $99.980 \%$ \\
\hline 1270 & *24:02 & *15:07 & *04:10 & $0.006 \%$ & $0.001 \%$ & 0.002 & $99.980 \%$ \\
\hline 1270 & $\star 24: 02$ & $\star 15: 07$ & ${ }^{\star} 08: 02$ & $0.006 \%$ & $-0.003 \%$ & -0.006 & $99.980 \%$ \\
\hline 1270 & *24:02 & *15:07 & *11:01 & $0.006 \%$ & $0.000 \%$ & 0.000 & $99.980 \%$ \\
\hline
\end{tabular}




\begin{tabular}{|c|c|c|c|c|c|c|c|}
\hline 1270 & $\star 24: 02$ & *15:07 & *13:02 & $0.006 \%$ & $-0.006 \%$ & -0.010 & $99.980 \%$ \\
\hline 1270 & *24:02 & *15:07 & *14:05 & $0.006 \%$ & $0.002 \%$ & 0.003 & $99.980 \%$ \\
\hline 1270 & *24:02 & *15:11 & ${ }^{\star} 04: 10$ & $0.006 \%$ & $-0.002 \%$ & -0.002 & $99.980 \%$ \\
\hline 1270 & *24:02 & *15:11 & *12:01 & $0.006 \%$ & $-0.007 \%$ & -0.007 & $99.980 \%$ \\
\hline 1270 & *24:02 & *15:11 & *13:02 & $0.006 \%$ & $-0.014 \%$ & -0.014 & $99.980 \%$ \\
\hline 1270 & *24:02 & *15:11 & *14:02 & $0.006 \%$ & $0.006 \%$ & 0.087 & $99.981 \%$ \\
\hline 1270 & *24:02 & *15:11 & *15:01 & $0.006 \%$ & $-0.022 \%$ & -0.023 & $99.981 \%$ \\
\hline 1270 & *24:02 & *15:18 & ${ }^{\star} 01: 01$ & $0.006 \%$ & $-0.025 \%$ & -0.017 & $99.981 \%$ \\
\hline 1270 & *24:02 & *15:18 & *11:01 & $0.006 \%$ & $-0.009 \%$ & -0.006 & $99.981 \%$ \\
\hline 1270 & *24:02 & *15:18 & *12:01 & $0.006 \%$ & $-0.013 \%$ & -0.009 & $99.981 \%$ \\
\hline 1270 & *24:02 & *15:18 & *12:02 & $0.006 \%$ & $-0.004 \%$ & -0.003 & $99.981 \%$ \\
\hline 1270 & *24:02 & *15:18 & *13:07 & $0.006 \%$ & $0.006 \%$ & 0.496 & $99.981 \%$ \\
\hline 1270 & *24:02 & *15:18 & *14:05 & $0.006 \%$ & $-0.005 \%$ & -0.003 & $99.981 \%$ \\
\hline 1270 & *24:02 & *15:25 & *15:02 & $0.006 \%$ & $0.006 \%$ & 0.991 & $99.981 \%$ \\
\hline 1270 & *24:02 & *15:27 & *15:02 & $0.006 \%$ & $0.003 \%$ & 0.047 & $99.981 \%$ \\
\hline 1270 & *24:02 & $\star 15: 35$ & *15:02 & $0.006 \%$ & $0.005 \%$ & 0.477 & $99.981 \%$ \\
\hline 1270 & *24:02 & *15:38 & *11:01 & $0.006 \%$ & $0.006 \%$ & 0.327 & $99.981 \%$ \\
\hline 1270 & *24:02 & *27:04 & *11:01 & $0.006 \%$ & $0.004 \%$ & 0.019 & $99.981 \%$ \\
\hline 1270 & *24:02 & *35:01 & *13:01 & $0.006 \%$ & $-0.011 \%$ & -0.020 & $99.981 \%$ \\
\hline 1270 & *24:02 & *35:64 & *14:54 & $0.006 \%$ & $0.006 \%$ & 1.000 & $99.981 \%$ \\
\hline 1270 & *24:02 & *38:02 & *09:01 & $0.006 \%$ & $-0.009 \%$ & -0.033 & $99.981 \%$ \\
\hline 1270 & *24:02 & *38:02 & *14:04 & $0.006 \%$ & $0.006 \%$ & 1.006 & $99.982 \%$ \\
\hline 1270 & *24:02 & *38:02 & *14:06 & $0.006 \%$ & $0.005 \%$ & 0.016 & $99.982 \%$ \\
\hline 1270 & *24:02 & *38:02 & *15:02 & $0.006 \%$ & $-0.005 \%$ & -0.018 & $99.982 \%$ \\
\hline 1270 & *24:02 & *39:01 & *04:06 & $0.006 \%$ & $-0.034 \%$ & -0.011 & $99.982 \%$ \\
\hline 1270 & *24:02 & *39:01 & *12:02 & $0.006 \%$ & $-0.017 \%$ & -0.009 & $99.982 \%$ \\
\hline 1270 & *24:02 & *39:02 & *04:03 & $0.006 \%$ & $0.003 \%$ & 0.011 & $99.982 \%$ \\
\hline 1270 & *24:02 & *39:02 & *14:54 & $0.006 \%$ & $0.002 \%$ & 0.008 & $99.982 \%$ \\
\hline 1270 & *24:02 & *39:02 & *15:01 & $0.006 \%$ & $-0.002 \%$ & -0.007 & $99.982 \%$ \\
\hline 1270 & *24:02 & *39:04 & *08:02 & $0.006 \%$ & $0.003 \%$ & 0.013 & $99.982 \%$ \\
\hline 1270 & *24:02 & *40:02 & ${ }^{\star} 04: 04$ & $0.006 \%$ & $-0.002 \%$ & -0.007 & $99.982 \%$ \\
\hline 1270 & *24:02 & *40:02 & *04:07 & $0.006 \%$ & $-0.009 \%$ & -0.018 & $99.982 \%$ \\
\hline 1270 & *24:02 & *40:02 & *16:02 & $0.006 \%$ & $-0.023 \%$ & -0.024 & $99.982 \%$ \\
\hline 1270 & *24:02 & *40:03 & ${ }^{\star} 04: 05$ & $0.006 \%$ & $-0.011 \%$ & -0.034 & $99.982 \%$ \\
\hline 1270 & *24:02 & *40:03 & *04:07 & $0.006 \%$ & $0.005 \%$ & 0.015 & $99.982 \%$ \\
\hline 1270 & *24:02 & *40:03 & ${ }^{\star} 04: 10$ & $0.006 \%$ & $0.003 \%$ & 0.009 & $99.982 \%$ \\
\hline 1270 & $\star 24: 02$ & *40:03 & *13:02 & $0.006 \%$ & $-0.001 \%$ & -0.003 & $99.983 \%$ \\
\hline 1270 & *24:02 & $\star 40: 06$ & ${ }^{\star} 01: 01$ & $0.006 \%$ & $-0.090 \%$ & -0.020 & $99.983 \%$ \\
\hline 1270 & *24:02 & *40:06 & *04:04 & $0.006 \%$ & $0.001 \%$ & 0.005 & $99.983 \%$ \\
\hline 1270 & *24:02 & *40:06 & *08:09 & $0.006 \%$ & $0.005 \%$ & 0.110 & $99.983 \%$ \\
\hline 1270 & *24:02 & *40:06 & *13:02 & $0.006 \%$ & $-0.085 \%$ & -0.019 & $99.983 \%$ \\
\hline 1270 & *24:02 & *40:52 & *11:01 & $0.006 \%$ & $0.006 \%$ & 0.998 & $99.983 \%$ \\
\hline 1270 & *24:02 & $\star 44: 03$ & ${ }^{\star} 01: 01$ & $0.006 \%$ & $-0.125 \%$ & -0.022 & $99.983 \%$ \\
\hline 1270 & $\star 24: 02$ & *44:03 & ${ }^{\star} 08: 03$ & $0.006 \%$ & $-0.188 \%$ & -0.031 & $99.983 \%$ \\
\hline 1270 & $\star 24: 02$ & *44:03 & *15:01 & $0.006 \%$ & $-0.169 \%$ & -0.028 & $99.983 \%$ \\
\hline 1270 & $\star 24: 02$ & *45:01 & *11:01 & $0.006 \%$ & $0.006 \%$ & 0.998 & $99.983 \%$ \\
\hline 1270 & $\star 24: 02$ & *46:01 & ${ }^{\star} 01: 01$ & $0.006 \%$ & $-0.097 \%$ & -0.020 & $99.983 \%$ \\
\hline 1270 & $\star 24: 02$ & *46:01 & ${ }^{\star} 03: 01$ & $0.006 \%$ & $0.004 \%$ & 0.030 & $99.983 \%$ \\
\hline 1270 & $\star 24: 02$ & *46:01 & ${ }^{\star} 04: 06$ & $0.006 \%$ & $-0.050 \%$ & -0.016 & $99.983 \%$ \\
\hline 1270 & $\star 24: 02$ & *46:01 & *11:01 & $0.006 \%$ & $-0.043 \%$ & -0.016 & $99.983 \%$ \\
\hline 1270 & $\star 24: 02$ & $\star 46: 01$ & *12:01 & $0.006 \%$ & $-0.058 \%$ & -0.016 & $99.983 \%$ \\
\hline 1270 & $\star 24: 02$ & $\star 46: 01$ & *14:54 & $0.006 \%$ & $-0.055 \%$ & -0.016 & $99.983 \%$ \\
\hline 1270 & *24:02 & $\star 46: 01$ & *14:05 & $0.006 \%$ & $-0.029 \%$ & -0.015 & $99.983 \%$ \\
\hline 1270 & $\star 24: 02$ & *46:01 & *14:06 & $0.006 \%$ & $-0.017 \%$ & -0.013 & $99.983 \%$ \\
\hline
\end{tabular}




\begin{tabular}{|c|c|c|c|c|c|c|c|}
\hline 1270 & *24:02 & $\star 46: 02$ & $\star 12: 02$ & $0.006 \%$ & $0.006 \%$ & 0.994 & $99.983 \%$ \\
\hline 1270 & *24:02 & $\star 48: 01$ & $\star 13: 02$ & $0.006 \%$ & $-0.050 \%$ & -0.018 & $99.984 \%$ \\
\hline 1270 & *24:02 & $\star 48: 01$ & $\star 14: 02$ & $0.006 \%$ & $0.005 \%$ & 0.081 & $99.984 \%$ \\
\hline 1270 & *24:02 & *48:01 & *14:07 & $0.006 \%$ & $0.005 \%$ & 0.053 & $99.984 \%$ \\
\hline 1270 & *24:02 & $\star 50: 01$ & *13:01 & $0.006 \%$ & $0.006 \%$ & 0.495 & $99.984 \%$ \\
\hline 1270 & *24:02 & $\star 51: 01$ & ${ }^{*} 01: 02$ & $0.006 \%$ & $0.005 \%$ & 0.224 & $99.984 \%$ \\
\hline 1270 & *24:02 & $\star 51: 01$ & ${ }^{\star} 03: 01$ & $0.006 \%$ & $0.002 \%$ & 0.015 & $99.984 \%$ \\
\hline 1270 & *24:02 & *51:01 & *13:01 & $0.006 \%$ & $-0.014 \%$ & -0.024 & $99.984 \%$ \\
\hline 1270 & *24:02 & *51:01 & *13:02 & $0.006 \%$ & $-0.180 \%$ & -0.034 & $99.984 \%$ \\
\hline 1270 & *24:02 & $\star 51: 01$ & *14:12 & $0.006 \%$ & $0.005 \%$ & 0.172 & $99.984 \%$ \\
\hline 1270 & *24:02 & *51:02 & *04:05 & $0.006 \%$ & $-0.004 \%$ & -0.021 & $99.984 \%$ \\
\hline 1270 & *24:02 & *51:02 & *16:02 & $0.006 \%$ & $0.005 \%$ & 0.026 & $99.984 \%$ \\
\hline 1270 & *24:02 & *51:36 & *01:01 & $0.006 \%$ & $0.006 \%$ & 1.009 & $99.984 \%$ \\
\hline 1270 & *24:02 & *52:01 & *04:07 & $0.006 \%$ & $-0.015 \%$ & -0.030 & $99.984 \%$ \\
\hline 1270 & *24:02 & $\star 52: 01$ & *10:01 & $0.006 \%$ & $-0.018 \%$ & -0.032 & $99.984 \%$ \\
\hline 1270 & *24:02 & *52:01 & *14:06 & $0.006 \%$ & $-0.046 \%$ & -0.038 & $99.984 \%$ \\
\hline 1270 & *24:02 & *52:01 & *16:02 & $0.006 \%$ & $-0.034 \%$ & -0.036 & $99.984 \%$ \\
\hline 1270 & *24:02 & *52:11 & *15:02 & $0.006 \%$ & $0.006 \%$ & 0.991 & $99.984 \%$ \\
\hline 1270 & *24:02 & $\star 54: 01$ & ${ }^{*} 04: 08$ & $0.006 \%$ & $0.006 \%$ & 0.997 & $99.984 \%$ \\
\hline 1270 & *24:02 & $\star 54: 01$ & ${ }^{*} 07: 01$ & $0.006 \%$ & $-0.006 \%$ & -0.014 & $99.984 \%$ \\
\hline 1270 & *24:02 & $\star 54: 01$ & ${ }^{\star} 08: 09$ & $0.006 \%$ & $0.005 \%$ & 0.100 & $99.984 \%$ \\
\hline 1270 & *24:02 & $\star 55: 02$ & ${ }^{\star} 01: 01$ & $0.006 \%$ & $-0.046 \%$ & -0.019 & $99.984 \%$ \\
\hline 1270 & *24:02 & $\star 55: 02$ & ${ }^{\star} 04: 03$ & $0.006 \%$ & $-0.019 \%$ & -0.008 & $99.984 \%$ \\
\hline 1270 & *24:02 & $\star 55: 02$ & ${ }^{\star} 04: 07$ & $0.006 \%$ & $0.001 \%$ & 0.003 & $99.984 \%$ \\
\hline 1270 & *24:02 & $\star 55: 02$ & *13:02 & $0.006 \%$ & $-0.043 \%$ & -0.018 & $99.984 \%$ \\
\hline 1270 & *24:02 & $\star 55: 02$ & *14:06 & $0.006 \%$ & $-0.006 \%$ & -0.004 & $99.984 \%$ \\
\hline 1270 & *24:02 & $\star 55: 02$ & *14:29 & $0.006 \%$ & $0.006 \%$ & 0.245 & $99.984 \%$ \\
\hline 1270 & *24:02 & $\star 55: 10$ & *15:01 & $0.006 \%$ & $0.006 \%$ & 0.998 & $99.984 \%$ \\
\hline 1270 & *24:02 & $\star 56: 01$ & ${ }^{*} 04: 03$ & $0.006 \%$ & $-0.003 \%$ & -0.004 & $99.984 \%$ \\
\hline 1270 & *24:02 & $\star 56: 01$ & *14:54 & $0.006 \%$ & $-0.006 \%$ & -0.006 & $99.985 \%$ \\
\hline 1270 & *24:02 & $\star 56: 01$ & *14:05 & $0.006 \%$ & $-0.001 \%$ & -0.001 & $99.985 \%$ \\
\hline 1270 & *24:02 & *58:01 & ${ }^{\star} 09: 01$ & $0.006 \%$ & $-0.026 \%$ & -0.045 & $99.985 \%$ \\
\hline 1270 & *24:02 & *59:01 & ${ }^{*} 01: 01$ & $0.006 \%$ & $-0.034 \%$ & -0.018 & $99.985 \%$ \\
\hline 1270 & *24:02 & *59:01 & ${ }^{\star} 04: 06$ & $0.006 \%$ & $-0.016 \%$ & -0.009 & $99.985 \%$ \\
\hline 1270 & *24:02 & *59:01 & *11:01 & $0.006 \%$ & $-0.014 \%$ & -0.007 & $99.985 \%$ \\
\hline 1270 & *24:02 & *59:01 & *14:03 & $0.006 \%$ & $-0.005 \%$ & -0.003 & $99.985 \%$ \\
\hline 1270 & *24:02 & $\star 59: 01$ & *14:06 & $0.006 \%$ & $-0.003 \%$ & -0.002 & $99.985 \%$ \\
\hline 1270 & $\star 24: 02$ & *67:01 & $\star 12: 01$ & $0.006 \%$ & $-0.009 \%$ & -0.008 & $99.985 \%$ \\
\hline 1270 & $\star 24: 02$ & *67:01 & $\star 14: 54$ & $0.006 \%$ & $-0.009 \%$ & -0.008 & $99.985 \%$ \\
\hline 1270 & $\star 24: 02$ & *67:01 & $\star 15: 02$ & $0.006 \%$ & $-0.038 \%$ & -0.035 & $99.985 \%$ \\
\hline 1270 & *24:02 & $\star 78: 02$ & ${ }^{\star} 04: 04$ & $0.006 \%$ & $0.006 \%$ & 1.006 & $99.985 \%$ \\
\hline 1270 & *24:03 & $\star 51: 06$ & ${ }^{\star} 07: 01$ & $0.006 \%$ & $0.006 \%$ & 1.005 & $99.985 \%$ \\
\hline 1270 & *24:03 & $\star 52: 01$ & *11:06 & $0.006 \%$ & $0.006 \%$ & 0.502 & $99.985 \%$ \\
\hline 1270 & *24:04 & *46:01 & ${ }^{*} 09: 01$ & $0.006 \%$ & $0.006 \%$ & 0.497 & $99.985 \%$ \\
\hline 1270 & *24:04 & $\star 52: 01$ & ${ }^{*} 08: 02$ & $0.006 \%$ & $0.006 \%$ & 0.496 & $99.986 \%$ \\
\hline 1270 & *24:07 & $\star 35: 05$ & ${ }^{*} 09: 01$ & $0.006 \%$ & $0.006 \%$ & 0.502 & $99.986 \%$ \\
\hline 1270 & *24:07 & *40:01 & ${ }^{*} 04: 05$ & $0.006 \%$ & $0.006 \%$ & 0.497 & $99.986 \%$ \\
\hline 1270 & *24:08 & *15:01 & ${ }^{*} 09: 01$ & $0.006 \%$ & $0.006 \%$ & 0.241 & $99.986 \%$ \\
\hline 1270 & *24:08 & *40:02 & ${ }^{\star} 08: 02$ & $0.006 \%$ & $0.006 \%$ & 0.248 & $99.986 \%$ \\
\hline 1270 & *24:08 & *44:03 & *14:05 & $0.006 \%$ & $0.006 \%$ & 0.247 & $99.986 \%$ \\
\hline 1270 & *24:08 & $\star 54: 01$ & *04:05 & $0.006 \%$ & $0.006 \%$ & 0.241 & $99.986 \%$ \\
\hline 1270 & *24:20 & $\star 07: 02$ & *01:01 & $0.006 \%$ & $0.004 \%$ & 0.005 & $99.986 \%$ \\
\hline 1270 & *24:20 & *13:01 & *14:03 & $0.006 \%$ & $0.006 \%$ & 0.008 & $99.986 \%$ \\
\hline 1270 & *24:20 & *13:01 & *15:02 & $0.006 \%$ & $0.005 \%$ & 0.007 & $99.986 \%$ \\
\hline
\end{tabular}




\begin{tabular}{|c|c|c|c|c|c|c|c|}
\hline 1270 & $\star 24: 20$ & *15:01 & ${ }^{\star} 04: 03$ & $0.006 \%$ & $0.004 \%$ & 0.006 & $99.987 \%$ \\
\hline 1270 & *24:20 & *15:01 & ${ }^{*} 09: 01$ & $0.006 \%$ & $-0.002 \%$ & -0.003 & $99.987 \%$ \\
\hline 1270 & $\star 24: 20$ & *15:01 & *14:06 & $0.006 \%$ & $0.005 \%$ & 0.007 & $99.987 \%$ \\
\hline 1270 & *24:20 & *15:18 & ${ }^{\star} 09: 01$ & $0.006 \%$ & $0.004 \%$ & 0.006 & $99.987 \%$ \\
\hline 1270 & *24:20 & *35:01 & ${ }^{\star} 08: 02$ & $0.006 \%$ & $0.003 \%$ & 0.005 & $99.987 \%$ \\
\hline 1270 & *24:20 & *35:01 & ${ }^{\star} 12: 01$ & $0.006 \%$ & $0.004 \%$ & 0.006 & $99.987 \%$ \\
\hline 1270 & *24:20 & *35:01 & *13:02 & $0.006 \%$ & $0.003 \%$ & 0.004 & $99.987 \%$ \\
\hline 1270 & *24:20 & *39:04 & ${ }^{*} 04: 03$ & $0.006 \%$ & $0.006 \%$ & 0.027 & $99.987 \%$ \\
\hline 1270 & *24:20 & *40:01 & ${ }^{*} 09: 01$ & $0.006 \%$ & $0.001 \%$ & 0.001 & $99.987 \%$ \\
\hline 1270 & *24:20 & *40:01 & *14:54 & $0.006 \%$ & $0.005 \%$ & 0.007 & $99.987 \%$ \\
\hline 1270 & *24:20 & *40:01 & *15:01 & $0.006 \%$ & $0.003 \%$ & 0.004 & $99.987 \%$ \\
\hline 1270 & *24:20 & *40:02 & ${ }^{*} 04: 06$ & $0.006 \%$ & $0.004 \%$ & 0.006 & $99.987 \%$ \\
\hline 1270 & *24:20 & *40:02 & ${ }^{*} 08: 02$ & $0.006 \%$ & $0.003 \%$ & 0.005 & $99.987 \%$ \\
\hline 1270 & *24:20 & *40:02 & *14:05 & $0.006 \%$ & $0.005 \%$ & 0.007 & $99.987 \%$ \\
\hline 1270 & *24:20 & *44:03 & *16:02 & $0.006 \%$ & $0.005 \%$ & 0.008 & $99.988 \%$ \\
\hline 1270 & *24:20 & *46:01 & ${ }^{*} 04: 05$ & $0.006 \%$ & $0.001 \%$ & 0.002 & $99.988 \%$ \\
\hline 1270 & *24:20 & *46:01 & *12:01 & $0.006 \%$ & $0.005 \%$ & 0.007 & $99.988 \%$ \\
\hline 1270 & *24:20 & *48:01 & *09:01 & $0.006 \%$ & $0.003 \%$ & 0.004 & $99.988 \%$ \\
\hline 1270 & $\star 24: 20$ & *51:01 & ${ }^{\star} 08: 02$ & $0.006 \%$ & $0.003 \%$ & 0.005 & $99.988 \%$ \\
\hline 1270 & $\star 24: 20$ & *51:01 & ${ }^{\star} 09: 01$ & $0.006 \%$ & $-0.003 \%$ & -0.005 & $99.988 \%$ \\
\hline 1270 & $\star 24: 20$ & *51:01 & ${ }^{\star} 12: 01$ & $0.006 \%$ & $0.004 \%$ & 0.005 & $99.988 \%$ \\
\hline 1270 & $\star 24: 20$ & *51:01 & ${ }^{\star} 14: 54$ & $0.006 \%$ & $0.004 \%$ & 0.005 & $99.988 \%$ \\
\hline 1270 & $\star 24: 20$ & *51:01 & ${ }^{\star} 14: 05$ & $0.006 \%$ & $0.005 \%$ & 0.007 & $99.988 \%$ \\
\hline 1270 & $\star 24: 20$ & *52:01 & ${ }^{\star} 15: 02$ & $0.006 \%$ & $-0.002 \%$ & -0.004 & $99.988 \%$ \\
\hline 1270 & *24:20 & *54:01 & *15:01 & $0.006 \%$ & $0.002 \%$ & 0.003 & $99.988 \%$ \\
\hline 1270 & *24:20 & *55:02 & ${ }^{*} 04: 03$ & $0.006 \%$ & $0.005 \%$ & 0.008 & $99.988 \%$ \\
\hline 1270 & *24:20 & *55:02 & *12:01 & $0.006 \%$ & $0.005 \%$ & 0.008 & $99.988 \%$ \\
\hline 1270 & *24:20 & *55:02 & *14:05 & $0.006 \%$ & $0.005 \%$ & 0.008 & $99.988 \%$ \\
\hline 1270 & *24:20 & *56:01 & ${ }^{\star} 08: 02$ & $0.006 \%$ & $0.006 \%$ & 0.008 & $99.989 \%$ \\
\hline 1270 & *26:01 & ${ }^{*} 07: 02$ & *13:02 & $0.006 \%$ & $-0.018 \%$ & -0.003 & $99.989 \%$ \\
\hline 1270 & *26:01 & *14:01 & ${ }^{*} 07: 01$ & $0.006 \%$ & $0.006 \%$ & 0.251 & $99.989 \%$ \\
\hline 1270 & *26:01 & *15:01 & ${ }^{*} 08: 02$ & $0.006 \%$ & $-0.020 \%$ & -0.005 & $99.989 \%$ \\
\hline 1270 & *26:01 & *15:01 & *11:01 & $0.006 \%$ & $-0.011 \%$ & -0.004 & $99.989 \%$ \\
\hline 1270 & *26:01 & *15:01 & *12:01 & $0.006 \%$ & $-0.016 \%$ & -0.004 & $99.989 \%$ \\
\hline 1270 & *26:01 & *15:03 & ${ }^{*} 09: 01$ & $0.006 \%$ & $0.006 \%$ & 0.998 & $99.989 \%$ \\
\hline 1270 & *26:01 & *15:07 & ${ }^{*} 08: 02$ & $0.006 \%$ & $0.004 \%$ & 0.006 & $99.989 \%$ \\
\hline 1270 & $\star 26: 01$ & *15:11 & ${ }^{\star} 08: 02$ & $0.006 \%$ & $0.003 \%$ & 0.003 & $99.989 \%$ \\
\hline 1270 & $\star 26: 01$ & *15:11 & *11:01 & $0.006 \%$ & $0.004 \%$ & 0.004 & $99.989 \%$ \\
\hline 1270 & $\star 26: 01$ & *15:18 & ${ }^{\star} 04: 05$ & $0.006 \%$ & $-0.009 \%$ & -0.007 & $99.989 \%$ \\
\hline 1270 & $\star 26: 01$ & *15:18 & ${ }^{\star} 04: 10$ & $0.006 \%$ & $0.003 \%$ & 0.002 & $99.989 \%$ \\
\hline 1270 & $\star 26: 01$ & *15:18 & *13:02 & $0.006 \%$ & $0.000 \%$ & 0.000 & $99.989 \%$ \\
\hline 1270 & *26:01 & *15:18 & ${ }^{\star} 15: 02$ & $0.006 \%$ & $-0.006 \%$ & -0.004 & $99.989 \%$ \\
\hline 1270 & *26:01 & *37:01 & *14:54 & $0.006 \%$ & $0.004 \%$ & 0.006 & $99.989 \%$ \\
\hline 1270 & *26:01 & *38:01 & ${ }^{\star} 01: 01$ & $0.006 \%$ & $0.006 \%$ & 0.195 & $99.990 \%$ \\
\hline 1270 & *26:01 & *38:01 & ${ }^{\star} 09: 01$ & $0.006 \%$ & $0.006 \%$ & 0.193 & $99.990 \%$ \\
\hline 1270 & *26:01 & *39:01 & ${ }^{\star} 04: 06$ & $0.006 \%$ & $-0.003 \%$ & -0.001 & $99.990 \%$ \\
\hline 1270 & *26:01 & *39:01 & ${ }^{\star} 04: 10$ & $0.006 \%$ & $0.000 \%$ & 0.000 & $99.990 \%$ \\
\hline 1270 & *26:01 & *39:01 & ${ }^{*} 08: 09$ & $0.006 \%$ & $0.006 \%$ & 0.124 & $99.990 \%$ \\
\hline 1270 & *26:01 & *39:02 & *14:54 & $0.006 \%$ & $0.005 \%$ & 0.018 & $99.990 \%$ \\
\hline 1270 & *26:01 & *40:01 & ${ }^{*} 01: 01$ & $0.006 \%$ & $-0.018 \%$ & -0.003 & $99.990 \%$ \\
\hline 1270 & *26:01 & *40:01 & ${ }^{*} 04: 03$ & $0.006 \%$ & $-0.006 \%$ & -0.002 & $99.990 \%$ \\
\hline 1270 & *26:01 & *40:01 & ${ }^{*} 04: 06$ & $0.006 \%$ & $-0.007 \%$ & -0.002 & $99.990 \%$ \\
\hline 1270 & *26:01 & *40:01 & ${ }^{*} 08: 09$ & $0.006 \%$ & $0.006 \%$ & 0.122 & $99.990 \%$ \\
\hline 1270 & $\star 26: 01$ & *40:02 & ${ }^{\star} 04: 01$ & $0.006 \%$ & $0.000 \%$ & 0.000 & $99.990 \%$ \\
\hline
\end{tabular}




\begin{tabular}{|c|c|c|c|c|c|c|c|}
\hline 1270 & *26:01 & $\star 40: 02$ & ${ }^{\star} 04: 04$ & $0.006 \%$ & $0.004 \%$ & 0.016 & $99.990 \%$ \\
\hline 1270 & *26:01 & $\star 40: 02$ & ${ }^{\star} 04: 06$ & $0.006 \%$ & $-0.014 \%$ & -0.004 & $99.990 \%$ \\
\hline 1270 & *26:01 & $\star 40: 02$ & *12:05 & $0.006 \%$ & $0.006 \%$ & 0.331 & $99.990 \%$ \\
\hline 1270 & *26:01 & *40:03 & ${ }^{\star} 04: 05$ & $0.006 \%$ & $0.002 \%$ & 0.007 & $99.990 \%$ \\
\hline 1270 & *26:01 & $\star 40: 03$ & ${ }^{\star} 08: 03$ & $0.006 \%$ & $0.004 \%$ & 0.010 & $99.990 \%$ \\
\hline 1270 & *26:01 & $\star 40: 06$ & ${ }^{\star} 04: 03$ & $0.006 \%$ & $-0.004 \%$ & -0.001 & $99.990 \%$ \\
\hline 1270 & $\star 26: 01$ & *40:06 & *14:05 & $0.006 \%$ & $-0.001 \%$ & -0.001 & $99.990 \%$ \\
\hline 1270 & $\star 26: 01$ & $\star 40: 06$ & $\star 15: 02$ & $0.006 \%$ & $-0.031 \%$ & -0.007 & $99.990 \%$ \\
\hline 1270 & $\star 26: 01$ & $\star 40: 11$ & $\star 09: 01$ & $0.006 \%$ & $0.006 \%$ & 0.998 & $99.990 \%$ \\
\hline 1270 & $\star 26: 01$ & $\star 44: 03$ & ${ }^{\star} 08: 03$ & $0.006 \%$ & $-0.035 \%$ & -0.006 & $99.990 \%$ \\
\hline 1270 & $\star 26: 01$ & $\star 46: 01$ & ${ }^{\star} 04: 03$ & $0.006 \%$ & $-0.005 \%$ & -0.002 & $99.990 \%$ \\
\hline 1270 & $\star 26: 01$ & $\star 46: 01$ & ${ }^{\star} 04: 05$ & $0.006 \%$ & $-0.045 \%$ & -0.009 & $99.991 \%$ \\
\hline 1270 & $\star 26: 01$ & $\star 46: 01$ & ${ }^{\star} 08: 02$ & $0.006 \%$ & $-0.010 \%$ & -0.002 & $99.991 \%$ \\
\hline 1270 & $\star 26: 01$ & $\star 46: 01$ & $\star 09: 01$ & $0.006 \%$ & $-0.048 \%$ & -0.010 & $99.991 \%$ \\
\hline 1270 & $\star 26: 01$ & *48:01 & ${ }^{*} 04: 07$ & $0.006 \%$ & $0.005 \%$ & 0.009 & $99.991 \%$ \\
\hline 1270 & *26:01 & *48:01 & ${ }^{\star} 04: 10$ & $0.006 \%$ & $0.001 \%$ & 0.001 & $99.991 \%$ \\
\hline 1270 & *26:01 & *48:01 & *12:01 & $0.006 \%$ & $-0.002 \%$ & -0.001 & $99.991 \%$ \\
\hline 1270 & *26:01 & *48:01 & *14:06 & $0.006 \%$ & $0.003 \%$ & 0.002 & $99.991 \%$ \\
\hline 1270 & *26:01 & *48:01 & *15:02 & $0.006 \%$ & $-0.017 \%$ & -0.006 & $99.991 \%$ \\
\hline 1270 & *26:01 & *49:01 & *07:01 & $0.006 \%$ & $0.006 \%$ & 1.005 & $99.991 \%$ \\
\hline 1270 & *26:01 & *51:01 & ${ }^{*} 04: 01$ & $0.006 \%$ & $-0.001 \%$ & -0.001 & $99.991 \%$ \\
\hline 1270 & *26:01 & *51:01 & *16:02 & $0.006 \%$ & $-0.001 \%$ & -0.001 & $99.991 \%$ \\
\hline 1270 & *26:01 & *51:02 & ${ }^{\star} 09: 01$ & $0.006 \%$ & $0.004 \%$ & 0.019 & $99.991 \%$ \\
\hline 1270 & *26:01 & $\star 52: 01$ & *13:02 & $0.006 \%$ & $-0.042 \%$ & -0.008 & $99.991 \%$ \\
\hline 1270 & $\star 26: 01$ & $\star 54: 01$ & ${ }^{\star} 08: 02$ & $0.006 \%$ & $-0.019 \%$ & -0.004 & $99.991 \%$ \\
\hline 1270 & $\star 26: 01$ & $\star 54: 01$ & *12:01 & $0.006 \%$ & $-0.015 \%$ & -0.004 & $99.991 \%$ \\
\hline 1270 & $\star 26: 01$ & $\star 54: 01$ & *14:03 & $0.006 \%$ & $-0.004 \%$ & -0.002 & $99.991 \%$ \\
\hline 1270 & $\star 26: 01$ & $\star 55: 02$ & ${ }^{\star} 04: 06$ & $0.006 \%$ & $0.000 \%$ & 0.000 & $99.991 \%$ \\
\hline 1270 & $\star 26: 01$ & $\star 55: 02$ & ${ }^{\star} 09: 01$ & $0.006 \%$ & $-0.021 \%$ & -0.009 & $99.991 \%$ \\
\hline 1270 & $\star 26: 01$ & $\star 55: 02$ & *12:01 & $0.006 \%$ & $-0.001 \%$ & 0.000 & $99.991 \%$ \\
\hline 1270 & *26:01 & $\star 55: 02$ & $\star 12: 02$ & $0.006 \%$ & $0.002 \%$ & 0.001 & $99.991 \%$ \\
\hline 1270 & $\star 26: 01$ & $\star 55: 04$ & $\star 09: 01$ & $0.006 \%$ & $0.004 \%$ & 0.033 & $99.991 \%$ \\
\hline 1270 & *26:01 & $\star 56: 01$ & ${ }^{\star} 04: 10$ & $0.006 \%$ & $0.004 \%$ & 0.005 & $99.991 \%$ \\
\hline 1270 & $\star 26: 01$ & $\star 59: 01$ & ${ }^{\star} 09: 01$ & $0.006 \%$ & $-0.015 \%$ & -0.008 & $99.991 \%$ \\
\hline 1270 & *26:01 & $\star 59: 01$ & $\star 15: 01$ & $0.006 \%$ & $-0.006 \%$ & -0.003 & $99.991 \%$ \\
\hline 1270 & *26:01 & *67:01 & ${ }^{\star} 08: 02$ & $0.006 \%$ & $0.002 \%$ & 0.002 & $99.992 \%$ \\
\hline 1270 & *26:02 & *15:01 & ${ }^{\star} 04: 03$ & $0.006 \%$ & $0.002 \%$ & 0.001 & $99.992 \%$ \\
\hline 1270 & *26:02 & *15:01 & $\star 04: 05$ & $0.006 \%$ & $-0.014 \%$ & -0.008 & $99.992 \%$ \\
\hline 1270 & $\star 26: 02$ & *15:01 & ${ }^{\star} 04: 06$ & $0.006 \%$ & $0.001 \%$ & 0.001 & $99.992 \%$ \\
\hline 1270 & *26:02 & *15:01 & ${ }^{*} 04: 07$ & $0.006 \%$ & $0.005 \%$ & 0.010 & $99.992 \%$ \\
\hline 1270 & *26:02 & *15:01 & *11:01 & $0.006 \%$ & $0.002 \%$ & 0.001 & $99.992 \%$ \\
\hline 1270 & *26:02 & *15:01 & *12:02 & $0.006 \%$ & $0.003 \%$ & 0.002 & $99.992 \%$ \\
\hline 1270 & *26:02 & *15:01 & *14:05 & $0.006 \%$ & $0.003 \%$ & 0.002 & $99.992 \%$ \\
\hline 1270 & *26:02 & *15:01 & *16:02 & $0.006 \%$ & $0.004 \%$ & 0.005 & $99.992 \%$ \\
\hline 1270 & *26:02 & *15:18 & ${ }^{\star} 04: 05$ & $0.006 \%$ & $0.002 \%$ & 0.001 & $99.992 \%$ \\
\hline 1270 & *26:02 & *35:01 & ${ }^{\star} 04: 03$ & $0.006 \%$ & $0.002 \%$ & 0.001 & $99.992 \%$ \\
\hline 1270 & *26:02 & *35:01 & ${ }^{\star} 04: 05$ & $0.006 \%$ & $-0.015 \%$ & -0.008 & $99.992 \%$ \\
\hline 1270 & $\star 26: 02$ & *35:01 & ${ }^{\star} 08: 03$ & $0.006 \%$ & $-0.007 \%$ & -0.004 & $99.992 \%$ \\
\hline 1270 & $\star 26: 02$ & *35:01 & $\star 14: 05$ & $0.006 \%$ & $0.003 \%$ & 0.002 & $99.992 \%$ \\
\hline 1270 & $\star 26: 02$ & $\star 35: 01$ & $\star 15: 02$ & $0.006 \%$ & $-0.010 \%$ & -0.006 & $99.992 \%$ \\
\hline 1270 & *26:02 & *39:01 & *14:54 & $0.006 \%$ & $0.004 \%$ & 0.002 & $99.992 \%$ \\
\hline 1270 & *26:02 & *39:04 & $\star 04: 05$ & $0.006 \%$ & $0.005 \%$ & 0.025 & $99.993 \%$ \\
\hline 1270 & $\star 26: 02$ & $\star 39: 04$ & $\star 15: 01$ & $0.006 \%$ & $0.006 \%$ & 0.027 & $99.993 \%$ \\
\hline 1270 & $\star 26: 02$ & *40:02 & ${ }^{\star} 08: 02$ & $0.006 \%$ & $-0.001 \%$ & 0.000 & $99.993 \%$ \\
\hline
\end{tabular}




\begin{tabular}{|c|c|c|c|c|c|c|c|}
\hline 1270 & *26:02 & $\star 40: 02$ & *11:01 & $0.006 \%$ & $0.002 \%$ & 0.001 & $99.993 \%$ \\
\hline 1270 & *26:02 & $\star 40: 02$ & *14:07 & $0.006 \%$ & $0.006 \%$ & 0.061 & $99.993 \%$ \\
\hline 1270 & *26:02 & *40:06 & *04:05 & $0.006 \%$ & $-0.006 \%$ & -0.003 & $99.993 \%$ \\
\hline 1270 & *26:02 & *40:06 & ${ }^{*} 08: 02$ & $0.006 \%$ & $0.002 \%$ & 0.001 & $99.993 \%$ \\
\hline 1270 & $\star 26: 02$ & $\star 40: 06$ & *13:02 & $0.006 \%$ & $0.001 \%$ & 0.001 & $99.993 \%$ \\
\hline 1270 & *26:02 & *46:01 & ${ }^{*} 04: 03$ & $0.006 \%$ & $0.003 \%$ & 0.002 & $99.993 \%$ \\
\hline 1270 & *26:02 & *46:01 & *11:01 & $0.006 \%$ & $0.003 \%$ & 0.002 & $99.993 \%$ \\
\hline 1270 & *26:02 & *48:01 & ${ }^{*} 04: 05$ & $0.006 \%$ & $-0.001 \%$ & -0.001 & $99.993 \%$ \\
\hline 1270 & *26:02 & *48:01 & *15:01 & $0.006 \%$ & $0.002 \%$ & 0.001 & $99.993 \%$ \\
\hline 1270 & *26:02 & $\star 51: 01$ & ${ }^{*} 04: 03$ & $0.006 \%$ & $0.001 \%$ & 0.001 & $99.993 \%$ \\
\hline 1270 & *26:02 & $\star 51: 01$ & *04:05 & $0.006 \%$ & $-0.018 \%$ & -0.010 & $99.993 \%$ \\
\hline 1270 & *26:02 & *51:01 & ${ }^{\star} 08: 02$ & $0.006 \%$ & $-0.002 \%$ & -0.001 & $99.993 \%$ \\
\hline 1270 & *26:02 & $\star 51: 01$ & *11:01 & $0.006 \%$ & $0.001 \%$ & 0.000 & $99.993 \%$ \\
\hline 1270 & *26:02 & *51:01 & *14:03 & $0.006 \%$ & $0.003 \%$ & 0.002 & $99.993 \%$ \\
\hline 1270 & *26:02 & *51:01 & *16:02 & $0.006 \%$ & $0.004 \%$ & 0.004 & $99.993 \%$ \\
\hline 1270 & *26:02 & *54:01 & *04:05 & $0.006 \%$ & $-0.013 \%$ & -0.007 & $99.993 \%$ \\
\hline 1270 & *26:02 & *54:01 & ${ }^{*} 09: 01$ & $0.006 \%$ & $-0.014 \%$ & -0.008 & $99.994 \%$ \\
\hline 1270 & *26:02 & *55:02 & ${ }^{*} 04: 03$ & $0.006 \%$ & $0.005 \%$ & 0.002 & $99.994 \%$ \\
\hline 1270 & *26:02 & $\star 55: 02$ & ${ }^{\star} 08: 03$ & $0.006 \%$ & $0.002 \%$ & 0.001 & $99.994 \%$ \\
\hline 1270 & $\star 26: 02$ & $\star 56: 03$ & *12:01 & $0.006 \%$ & $0.006 \%$ & 0.025 & $99.994 \%$ \\
\hline 1270 & *26:02 & *67:01 & ${ }^{*} 04: 10$ & $0.006 \%$ & $0.005 \%$ & 0.005 & $99.994 \%$ \\
\hline 1270 & *26:02 & *67:01 & *16:02 & $0.006 \%$ & $0.006 \%$ & 0.006 & $99.994 \%$ \\
\hline 1270 & *26:03 & ${ }^{*} 07: 02$ & ${ }^{\star} 09: 01$ & $0.006 \%$ & $-0.013 \%$ & -0.006 & $99.994 \%$ \\
\hline 1270 & *26:03 & ${ }^{*} 07: 02$ & *11:01 & $0.006 \%$ & $0.002 \%$ & 0.001 & $99.994 \%$ \\
\hline 1270 & *26:03 & $\star 15: 01$ & ${ }^{*} 08: 03$ & $0.006 \%$ & $-0.009 \%$ & -0.004 & $99.994 \%$ \\
\hline 1270 & *26:03 & *15:01 & $\star 13: 02$ & $0.006 \%$ & $-0.004 \%$ & -0.002 & $99.994 \%$ \\
\hline 1270 & $\star 26: 03$ & *15:01 & *14:03 & $0.006 \%$ & $0.003 \%$ & 0.002 & $99.994 \%$ \\
\hline 1270 & *26:03 & *15:07 & ${ }^{\star} 04: 03$ & $0.006 \%$ & $0.005 \%$ & 0.009 & $99.994 \%$ \\
\hline 1270 & *26:03 & *27:04 & ${ }^{\star} 04: 05$ & $0.006 \%$ & $0.005 \%$ & 0.025 & $99.994 \%$ \\
\hline 1270 & *26:03 & *35:01 & *15:02 & $0.006 \%$ & $-0.014 \%$ & -0.006 & $99.994 \%$ \\
\hline 1270 & *26:03 & *35:01 & *16:02 & $0.006 \%$ & $0.004 \%$ & 0.004 & $99.994 \%$ \\
\hline 1270 & *26:03 & *37:01 & *10:01 & $0.006 \%$ & $0.006 \%$ & 0.010 & $99.995 \%$ \\
\hline 1270 & *26:03 & *39:01 & ${ }^{\star} 04: 06$ & $0.006 \%$ & $0.003 \%$ & 0.001 & $99.995 \%$ \\
\hline 1270 & *26:03 & *39:01 & *08:03 & $0.006 \%$ & $-0.001 \%$ & 0.000 & $99.995 \%$ \\
\hline 1270 & *26:03 & *39:01 & *15:02 & $0.006 \%$ & $-0.003 \%$ & -0.001 & $99.995 \%$ \\
\hline 1270 & *26:03 & *39:02 & *09:01 & $0.006 \%$ & $0.005 \%$ & 0.018 & $99.995 \%$ \\
\hline 1270 & $\star 26: 03$ & *40:01 & ${ }^{\star} 01: 01$ & $0.006 \%$ & $-0.001 \%$ & -0.001 & $99.995 \%$ \\
\hline 1270 & $\star 26: 03$ & *40:01 & ${ }^{\star} 04: 04$ & $0.006 \%$ & $0.006 \%$ & 0.021 & $99.995 \%$ \\
\hline 1270 & $\star 26: 03$ & *40:01 & ${ }^{\star} 08: 03$ & $0.006 \%$ & $-0.005 \%$ & -0.002 & $99.995 \%$ \\
\hline 1270 & $\star 26: 03$ & *40:01 & *11:01 & $0.006 \%$ & $0.002 \%$ & 0.001 & $99.995 \%$ \\
\hline 1270 & $\star 26: 03$ & *40:01 & $\star 12: 01$ & $0.006 \%$ & $0.001 \%$ & 0.001 & $99.995 \%$ \\
\hline 1270 & *26:03 & *40:01 & *13:02 & $0.006 \%$ & $-0.001 \%$ & 0.000 & $99.995 \%$ \\
\hline 1270 & $\star 26: 03$ & *40:01 & *14:03 & $0.006 \%$ & $0.004 \%$ & 0.002 & $99.995 \%$ \\
\hline 1270 & *26:03 & *40:01 & *15:01 & $0.006 \%$ & $-0.004 \%$ & -0.002 & $99.995 \%$ \\
\hline 1270 & $\star 26: 03$ & *40:02 & ${ }^{*} 04: 03$ & $0.006 \%$ & $0.001 \%$ & 0.000 & $99.995 \%$ \\
\hline 1270 & *26:03 & *40:02 & ${ }^{*} 04: 06$ & $0.006 \%$ & $0.000 \%$ & 0.000 & $99.995 \%$ \\
\hline 1270 & *26:03 & *40:02 & *11:01 & $0.006 \%$ & $0.001 \%$ & 0.000 & $99.995 \%$ \\
\hline 1270 & *26:03 & $\star 40: 02$ & *13:02 & $0.006 \%$ & $-0.004 \%$ & -0.002 & $99.995 \%$ \\
\hline 1270 & *26:03 & *40:02 & *14:03 & $0.006 \%$ & $0.003 \%$ & 0.002 & $99.995 \%$ \\
\hline 1270 & *26:03 & *40:02 & *14:05 & $0.006 \%$ & $0.002 \%$ & 0.001 & $99.995 \%$ \\
\hline 1270 & *26:03 & *40:02 & *14:45 & $0.006 \%$ & $0.006 \%$ & 1.007 & $99.995 \%$ \\
\hline 1270 & *26:03 & $\star 40: 02$ & *15:01 & $0.006 \%$ & $-0.008 \%$ & -0.004 & $99.995 \%$ \\
\hline 1270 & *26:03 & *40:03 & *15:01 & $0.006 \%$ & $0.005 \%$ & 0.015 & $99.995 \%$ \\
\hline 1270 & *26:03 & *40:06 & *12:01 & $0.006 \%$ & $0.002 \%$ & 0.001 & $99.996 \%$ \\
\hline
\end{tabular}




\begin{tabular}{|c|c|c|c|c|c|c|c|}
\hline 1270 & *26:03 & *44:03 & ${ }^{\star} 08: 03$ & $0.006 \%$ & $-0.006 \%$ & -0.003 & $99.996 \%$ \\
\hline 1270 & *26:03 & *44:03 & $\star 13: 02$ & $0.006 \%$ & $-0.002 \%$ & -0.001 & $99.996 \%$ \\
\hline 1270 & *26:03 & *46:01 & $\star 12: 02$ & $0.006 \%$ & $0.004 \%$ & 0.002 & $99.996 \%$ \\
\hline 1270 & $\star 26: 03$ & *48:01 & ${ }^{\star} 08: 03$ & $0.006 \%$ & $0.000 \%$ & 0.000 & $99.996 \%$ \\
\hline 1270 & $\star 26: 03$ & *48:01 & *14:05 & $0.006 \%$ & $0.005 \%$ & 0.002 & $99.996 \%$ \\
\hline 1270 & *26:03 & *51:01 & *11:01 & $0.006 \%$ & $0.000 \%$ & 0.000 & $99.996 \%$ \\
\hline 1270 & *26:03 & $\star 51: 01$ & *12:01 & $0.006 \%$ & $-0.002 \%$ & -0.001 & $99.996 \%$ \\
\hline 1270 & *26:03 & $\star 51: 01$ & *14:05 & $0.006 \%$ & $0.002 \%$ & 0.001 & $99.996 \%$ \\
\hline 1270 & *26:03 & $\star 54: 01$ & *08:03 & $0.006 \%$ & $-0.009 \%$ & -0.004 & $99.996 \%$ \\
\hline 1270 & *26:03 & $\star 54: 01$ & *09:01 & $0.006 \%$ & $-0.019 \%$ & -0.008 & $99.996 \%$ \\
\hline 1270 & *26:03 & $\star 54: 01$ & *13:01 & $0.006 \%$ & $0.005 \%$ & 0.008 & $99.996 \%$ \\
\hline 1270 & *26:03 & $\star 54: 01$ & *15:02 & $0.006 \%$ & $-0.013 \%$ & -0.006 & $99.996 \%$ \\
\hline 1270 & *26:03 & $\star 55: 02$ & ${ }^{*} 04: 05$ & $0.006 \%$ & $-0.002 \%$ & -0.001 & $99.996 \%$ \\
\hline 1270 & *26:03 & *55:02 & *15:02 & $0.006 \%$ & $0.000 \%$ & 0.000 & $99.996 \%$ \\
\hline 1270 & *26:03 & *59:01 & *15:02 & $0.006 \%$ & $0.001 \%$ & 0.001 & $99.996 \%$ \\
\hline 1270 & *26:05 & *48:01 & *15:01 & $0.006 \%$ & $0.006 \%$ & 0.075 & $99.996 \%$ \\
\hline 1270 & *26:05 & *51:01 & *04:03 & $0.006 \%$ & $0.006 \%$ & 0.075 & $99.996 \%$ \\
\hline 1270 & *26:05 & *51:01 & *10:01 & $0.006 \%$ & $0.006 \%$ & 0.076 & $99.996 \%$ \\
\hline 1270 & *26:05 & *51:01 & *12:01 & $0.006 \%$ & $0.006 \%$ & 0.074 & $99.996 \%$ \\
\hline 1270 & *26:05 & *51:01 & *14:06 & $0.006 \%$ & $0.006 \%$ & 0.076 & $99.996 \%$ \\
\hline 1270 & *26:05 & *55:02 & ${ }^{*} 04: 05$ & $0.006 \%$ & $0.006 \%$ & 0.074 & $99.996 \%$ \\
\hline 1270 & $\star 26: 06$ & *15:01 & *15:01 & $0.006 \%$ & $0.006 \%$ & 0.994 & $99.996 \%$ \\
\hline 1270 & *26:18 & *27:05 & ${ }^{\star} 04: 04$ & $0.006 \%$ & $0.006 \%$ & 1.005 & $99.996 \%$ \\
\hline 1270 & *29:01 & ${ }^{*} 07: 05$ & *15:01 & $0.006 \%$ & $0.006 \%$ & 0.167 & $99.997 \%$ \\
\hline 1270 & *29:01 & *40:03 & *12:01 & $0.006 \%$ & $0.006 \%$ & 0.167 & $99.997 \%$ \\
\hline 1270 & *29:02 & $\star 13: 02$ & *10:01 & $0.006 \%$ & $0.006 \%$ & 1.005 & $99.997 \%$ \\
\hline 1270 & *30:01 & ${ }^{*} 07: 02$ & ${ }^{*} 01: 01$ & $0.006 \%$ & $0.005 \%$ & 0.025 & $99.997 \%$ \\
\hline 1270 & *30:01 & $\star 07: 05$ & *04:05 & $0.006 \%$ & $0.006 \%$ & 0.126 & $99.997 \%$ \\
\hline 1270 & *30:01 & $\star 13: 02$ & *04:06 & $0.006 \%$ & $0.006 \%$ & 0.028 & $99.997 \%$ \\
\hline 1270 & *30:01 & $\star 13: 02$ & *09:01 & $0.006 \%$ & $0.006 \%$ & 0.027 & $99.997 \%$ \\
\hline 1270 & *30:01 & *13:02 & *13:01 & $0.006 \%$ & $0.006 \%$ & 0.028 & $99.997 \%$ \\
\hline 1270 & *30:01 & *13:02 & *14:06 & $0.006 \%$ & $0.006 \%$ & 0.028 & $99.997 \%$ \\
\hline 1270 & *30:01 & *53:01 & ${ }^{\star} 03: 01$ & $0.006 \%$ & $0.006 \%$ & 1.005 & $99.997 \%$ \\
\hline 1270 & *30:04 & *14:01 & ${ }^{\star} 07: 01$ & $0.006 \%$ & $0.006 \%$ & 0.251 & $99.997 \%$ \\
\hline 1270 & *30:04 & *35:01 & *12:01 & $0.006 \%$ & $0.006 \%$ & 0.165 & $99.997 \%$ \\
\hline 1270 & *30:04 & *40:06 & *08:03 & $0.006 \%$ & $0.006 \%$ & 0.162 & $99.997 \%$ \\
\hline 1270 & $\star 30: 04$ & *55:02 & *14:54 & $0.006 \%$ & $0.006 \%$ & 0.165 & $99.997 \%$ \\
\hline 1270 & *31:01 & $* 07: 02$ & ${ }^{\star} 04: 05$ & $0.006 \%$ & $-0.063 \%$ & -0.011 & $99.997 \%$ \\
\hline 1270 & $\star 31: 01$ & $\star 07: 02$ & ${ }^{\star} 09: 01$ & $0.006 \%$ & $-0.067 \%$ & -0.012 & $99.997 \%$ \\
\hline 1270 & *31:01 & $* 07: 02$ & *11:01 & $0.006 \%$ & $-0.008 \%$ & -0.003 & $99.997 \%$ \\
\hline 1270 & *31:01 & *13:01 & ${ }^{\star} 04: 05$ & $0.006 \%$ & $-0.009 \%$ & -0.007 & $99.997 \%$ \\
\hline 1270 & *31:01 & *13:01 & ${ }^{\star} 04: 10$ & $0.006 \%$ & $0.004 \%$ & 0.003 & $99.998 \%$ \\
\hline 1270 & *31:01 & *13:01 & ${ }^{\star} 08: 03$ & $0.006 \%$ & $-0.004 \%$ & -0.003 & $99.998 \%$ \\
\hline 1270 & *31:01 & *15:01 & ${ }^{\star} 04: 07$ & $0.006 \%$ & $0.002 \%$ & 0.005 & $99.998 \%$ \\
\hline 1270 & *31:01 & *15:01 & *12:01 & $0.006 \%$ & $-0.019 \%$ & -0.005 & $99.998 \%$ \\
\hline 1270 & *31:01 & *15:01 & *12:02 & $0.006 \%$ & $-0.007 \%$ & -0.004 & $99.998 \%$ \\
\hline 1270 & *31:01 & *15:01 & *14:02 & $0.006 \%$ & $0.005 \%$ & 0.084 & $99.998 \%$ \\
\hline 1270 & *31:01 & *15:01 & *14:05 & $0.006 \%$ & $-0.008 \%$ & -0.004 & $99.998 \%$ \\
\hline 1270 & *31:01 & *15:01 & *15:02 & $0.006 \%$ & $-0.068 \%$ & -0.009 & $99.998 \%$ \\
\hline 1270 & *31:01 & *15:07 & *09:01 & $0.006 \%$ & $-0.002 \%$ & -0.003 & $99.998 \%$ \\
\hline 1270 & *31:01 & *15:11 & ${ }^{\star} 08: 03$ & $0.006 \%$ & $-0.002 \%$ & -0.002 & $99.998 \%$ \\
\hline 1270 & *31:01 & *15:11 & *14:06 & $0.006 \%$ & $0.005 \%$ & 0.005 & $99.998 \%$ \\
\hline 1270 & *31:01 & *15:18 & ${ }^{\star} 08: 02$ & $0.006 \%$ & $0.000 \%$ & 0.000 & $99.998 \%$ \\
\hline 1270 & *31:01 & *27:04 & *15:01 & $0.006 \%$ & $0.004 \%$ & 0.022 & $99.998 \%$ \\
\hline
\end{tabular}




\begin{tabular}{|c|c|c|c|c|c|c|c|}
\hline 1270 & *31:01 & *27:05 & ${ }^{\star} 08: 03$ & $0.006 \%$ & $0.005 \%$ & 0.065 & $99.998 \%$ \\
\hline 1270 & *31:01 & *35:01 & ${ }^{\star} 01: 01$ & $0.006 \%$ & $-0.036 \%$ & -0.006 & $99.998 \%$ \\
\hline 1270 & *31:01 & *35:01 & *12:02 & $0.006 \%$ & $-0.007 \%$ & -0.004 & $99.998 \%$ \\
\hline 1270 & *31:01 & *35:01 & *13:02 & $0.006 \%$ & $-0.034 \%$ & -0.006 & $99.998 \%$ \\
\hline 1270 & *31:01 & *35:04 & ${ }^{\star} 04: 11$ & $0.006 \%$ & $0.006 \%$ & 1.005 & $99.998 \%$ \\
\hline 1270 & *31:01 & *37:01 & *08:03 & $0.006 \%$ & $0.001 \%$ & 0.001 & $99.998 \%$ \\
\hline 1270 & *31:01 & *37:01 & *11:01 & $0.006 \%$ & $0.004 \%$ & 0.007 & $99.998 \%$ \\
\hline 1270 & *31:01 & *39:01 & ${ }^{\star} 04: 06$ & $0.006 \%$ & $-0.004 \%$ & -0.001 & $99.998 \%$ \\
\hline 1270 & *31:01 & *39:01 & *11:01 & $0.006 \%$ & $-0.003 \%$ & -0.001 & $99.998 \%$ \\
\hline 1270 & *31:01 & *39:01 & *14:06 & $0.006 \%$ & $0.002 \%$ & 0.001 & $99.998 \%$ \\
\hline 1270 & *31:01 & *39:02 & *04:05 & $0.006 \%$ & $0.002 \%$ & 0.009 & $99.998 \%$ \\
\hline 1270 & *31:01 & *39:02 & ${ }^{\star} 04: 10$ & $0.006 \%$ & $0.005 \%$ & 0.019 & $99.998 \%$ \\
\hline 1270 & *31:01 & *39:02 & *14:06 & $0.006 \%$ & $0.006 \%$ & 0.020 & $99.998 \%$ \\
\hline 1270 & *31:01 & *39:04 & *04:05 & $0.006 \%$ & $0.003 \%$ & 0.016 & $99.998 \%$ \\
\hline 1270 & *31:01 & *39:23 & *09:01 & $0.006 \%$ & $0.006 \%$ & 1.000 & $99.998 \%$ \\
\hline 1270 & *31:01 & *40:01 & ${ }^{\star} 04: 03$ & $0.006 \%$ & $-0.007 \%$ & -0.003 & $99.998 \%$ \\
\hline 1270 & *31:01 & *40:01 & ${ }^{*} 04: 06$ & $0.006 \%$ & $-0.009 \%$ & -0.003 & $99.998 \%$ \\
\hline 1270 & *31:01 & *40:01 & *14:03 & $0.006 \%$ & $-0.002 \%$ & -0.001 & $99.998 \%$ \\
\hline 1270 & *31:01 & *40:01 & *14:06 & $0.006 \%$ & $0.000 \%$ & 0.000 & $99.998 \%$ \\
\hline 1270 & *31:01 & *40:02 & *14:02 & $0.006 \%$ & $0.005 \%$ & 0.084 & $99.998 \%$ \\
\hline 1270 & *31:01 & *40:02 & *14:06 & $0.006 \%$ & $-0.003 \%$ & -0.003 & $99.998 \%$ \\
\hline 1270 & *31:01 & *40:03 & *09:01 & $0.006 \%$ & $0.001 \%$ & 0.004 & $99.998 \%$ \\
\hline 1270 & *31:01 & *40:06 & *04:03 & $0.006 \%$ & $-0.005 \%$ & -0.002 & $99.999 \%$ \\
\hline 1270 & *31:01 & *40:06 & *14:03 & $0.006 \%$ & $-0.001 \%$ & 0.000 & $99.999 \%$ \\
\hline 1270 & *31:01 & *44:02 & *13:01 & $0.006 \%$ & $0.006 \%$ & 0.015 & $99.999 \%$ \\
\hline 1270 & *31:01 & *44:03 & *08:02 & $0.006 \%$ & $-0.018 \%$ & -0.004 & $99.999 \%$ \\
\hline 1270 & *31:01 & *46:01 & *04:05 & $0.006 \%$ & $-0.053 \%$ & -0.011 & $99.999 \%$ \\
\hline 1270 & *31:01 & *48:01 & *04:03 & $0.006 \%$ & $-0.001 \%$ & 0.000 & $99.999 \%$ \\
\hline 1270 & *31:01 & *48:01 & *04:05 & $0.006 \%$ & $-0.028 \%$ & -0.010 & $99.999 \%$ \\
\hline 1270 & *31:01 & *48:01 & *11:01 & $0.006 \%$ & $-0.001 \%$ & 0.000 & $99.999 \%$ \\
\hline 1270 & *31:01 & *48:01 & *12:01 & $0.006 \%$ & $-0.003 \%$ & -0.001 & $99.999 \%$ \\
\hline 1270 & *31:01 & *48:01 & *14:54 & $0.006 \%$ & $-0.003 \%$ & -0.001 & $99.999 \%$ \\
\hline 1270 & *31:01 & *48:01 & *14:05 & $0.006 \%$ & $0.001 \%$ & 0.000 & $99.999 \%$ \\
\hline 1270 & *31:01 & *48:01 & *16:02 & $0.006 \%$ & $0.003 \%$ & 0.003 & $99.999 \%$ \\
\hline 1270 & *31:01 & *51:01 & ${ }^{\star} 04: 04$ & $0.006 \%$ & $0.004 \%$ & 0.014 & $99.999 \%$ \\
\hline 1270 & *31:01 & $\star 51: 01$ & *04:07 & $0.006 \%$ & $0.002 \%$ & 0.003 & $99.999 \%$ \\
\hline 1270 & $\star 31: 01$ & *51:01 & *13:02 & $0.006 \%$ & $-0.040 \%$ & -0.007 & $99.999 \%$ \\
\hline 1270 & *31:01 & *51:02 & *04:03 & $0.006 \%$ & $0.005 \%$ & 0.027 & $99.999 \%$ \\
\hline 1270 & *31:01 & *51:02 & *04:05 & $0.006 \%$ & $0.003 \%$ & 0.018 & $99.999 \%$ \\
\hline 1270 & *31:01 & *51:02 & ${ }^{\star} 08: 02$ & $0.006 \%$ & $0.005 \%$ & 0.026 & $99.999 \%$ \\
\hline 1270 & *31:01 & *51:02 & *11:01 & $0.006 \%$ & $0.005 \%$ & 0.027 & $99.999 \%$ \\
\hline 1270 & *31:01 & $\star 51: 02$ & *15:02 & $0.006 \%$ & $0.004 \%$ & 0.020 & $99.999 \%$ \\
\hline 1270 & *31:01 & $\star 52: 01$ & *04:05 & $0.006 \%$ & $-0.131 \%$ & -0.015 & $99.999 \%$ \\
\hline 1270 & $\star 31: 01$ & *52:01 & *13:02 & $0.006 \%$ & $-0.050 \%$ & -0.009 & $99.999 \%$ \\
\hline 1270 & $\star 31: 01$ & *54:01 & ${ }^{\star} 12: 01$ & $0.006 \%$ & $-0.019 \%$ & -0.005 & $99.999 \%$ \\
\hline 1270 & $\star 31: 01$ & *55:02 & ${ }^{\star} 01: 01$ & $0.006 \%$ & $-0.007 \%$ & -0.003 & $99.999 \%$ \\
\hline 1270 & $\star 31: 01$ & *55:02 & ${ }^{\star} 04: 03$ & $0.006 \%$ & $0.000 \%$ & 0.000 & $99.999 \%$ \\
\hline 1270 & $\star 31: 01$ & *55:02 & *11:01 & $0.006 \%$ & $0.000 \%$ & 0.000 & $99.999 \%$ \\
\hline 1270 & $\star 31: 01$ & *55:02 & *13:02 & $0.006 \%$ & $-0.006 \%$ & -0.003 & $99.999 \%$ \\
\hline 1270 & $\star 31: 01$ & *55:02 & *15:01 & $0.006 \%$ & $-0.011 \%$ & -0.005 & $99.999 \%$ \\
\hline 1270 & $\star 31: 01$ & *56:01 & ${ }^{\star} 01: 01$ & $0.006 \%$ & $0.001 \%$ & 0.001 & $99.999 \%$ \\
\hline 1270 & $\star 31: 01$ & $\star 56: 01$ & ${ }^{\star} 04: 03$ & $0.006 \%$ & $0.004 \%$ & 0.004 & $99.999 \%$ \\
\hline 1270 & *31:01 & $\star 56: 01$ & *04:06 & $0.006 \%$ & $0.003 \%$ & 0.003 & $99.999 \%$ \\
\hline 1270 & *31:01 & *56:01 & ${ }^{\star} 08: 02$ & $0.006 \%$ & $0.002 \%$ & 0.003 & $99.999 \%$ \\
\hline
\end{tabular}




\begin{tabular}{|c|c|c|c|c|c|c|c|}
\hline 1270 & *31:01 & *56:01 & ${ }^{\star} 08: 03$ & $0.006 \%$ & $-0.001 \%$ & -0.001 & $99.999 \%$ \\
\hline 1270 & *31:01 & *56:01 & *11:01 & $0.006 \%$ & $0.004 \%$ & 0.004 & $99.999 \%$ \\
\hline 1270 & *31:01 & *56:01 & *12:01 & $0.006 \%$ & $0.003 \%$ & 0.003 & $99.999 \%$ \\
\hline 1270 & *31:01 & *56:01 & *14:54 & $0.006 \%$ & $0.003 \%$ & 0.003 & $99.999 \%$ \\
\hline 1270 & *31:01 & *56:01 & ${ }^{\star} 15: 02$ & $0.006 \%$ & $-0.003 \%$ & -0.003 & $99.999 \%$ \\
\hline 1270 & *31:01 & *56:03 & ${ }^{\star} 12: 01$ & $0.006 \%$ & $0.005 \%$ & 0.022 & $99.999 \%$ \\
\hline 1270 & *31:01 & $\star 58: 01$ & *13:02 & $0.006 \%$ & $0.003 \%$ & 0.005 & $99.999 \%$ \\
\hline 1270 & *31:01 & $\star 59: 01$ & ${ }^{*} 09: 01$ & $0.006 \%$ & $-0.019 \%$ & -0.010 & $99.999 \%$ \\
\hline 1270 & *31:01 & *67:01 & *16:02 & $0.006 \%$ & $0.005 \%$ & 0.005 & $99.999 \%$ \\
\hline 1270 & *31:11 & $\star 51: 01$ & ${ }^{*} 04: 07$ & $0.006 \%$ & $0.006 \%$ & 1.005 & $99.999 \%$ \\
\hline 1270 & *32:01 & $\star 15: 07$ & ${ }^{*} 04: 03$ & $0.006 \%$ & $0.006 \%$ & 0.168 & $99.999 \%$ \\
\hline 1270 & *32:01 & *44:02 & ${ }^{*} 04: 06$ & $0.006 \%$ & $0.006 \%$ & 0.167 & $99.999 \%$ \\
\hline 1270 & *32:01 & *44:02 & ${ }^{*} 09: 01$ & $0.006 \%$ & $0.006 \%$ & 0.168 & $99.999 \%$ \\
\hline 1270 & *32:01 & $\star 58: 01$ & *13:02 & $0.006 \%$ & $0.006 \%$ & 0.168 & $99.999 \%$ \\
\hline 1270 & *33:01 & *82:02 & ${ }^{*} 04: 03$ & $0.006 \%$ & $0.006 \%$ & 1.005 & $99.999 \%$ \\
\hline 1270 & *33:03 & *13:01 & *12:02 & $0.006 \%$ & $0.004 \%$ & 0.003 & $99.999 \%$ \\
\hline 1270 & *33:03 & *15:01 & ${ }^{*} 04: 10$ & $0.006 \%$ & $-0.006 \%$ & -0.003 & $99.999 \%$ \\
\hline 1270 & *33:03 & *15:01 & *14:54 & $0.006 \%$ & $-0.013 \%$ & -0.004 & $99.999 \%$ \\
\hline 1270 & *33:03 & *15:01 & *15:01 & $0.006 \%$ & $-0.036 \%$ & -0.005 & $99.999 \%$ \\
\hline 1270 & *33:03 & *15:11 & ${ }^{\star} 04: 05$ & $0.006 \%$ & $-0.004 \%$ & -0.004 & $99.999 \%$ \\
\hline 1270 & *33:03 & *15:18 & ${ }^{\star} 01: 01$ & $0.006 \%$ & $0.000 \%$ & 0.000 & $100.000 \%$ \\
\hline 1270 & *33:03 & *15:18 & ${ }^{\star} 04: 01$ & $0.006 \%$ & $0.005 \%$ & 0.005 & $100.000 \%$ \\
\hline 1270 & *33:03 & *35:01 & ${ }^{\star} 04: 03$ & $0.006 \%$ & $-0.010 \%$ & -0.004 & $100.000 \%$ \\
\hline 1270 & *33:03 & *35:01 & ${ }^{\star} 08: 02$ & $0.006 \%$ & $-0.018 \%$ & -0.004 & $100.000 \%$ \\
\hline 1270 & *33:03 & $\star 35: 01$ & ${ }^{*} 08: 03$ & $0.006 \%$ & $-0.043 \%$ & -0.006 & $100.000 \%$ \\
\hline 1270 & *33:03 & $* 35: 01$ & $\star 12: 01$ & $0.006 \%$ & $-0.015 \%$ & -0.004 & $100.000 \%$ \\
\hline 1270 & *33:03 & *39:01 & ${ }^{\star} 04: 10$ & $0.006 \%$ & $0.001 \%$ & 0.000 & $100.000 \%$ \\
\hline 1270 & *33:03 & *39:01 & ${ }^{*} 09: 01$ & $0.006 \%$ & $-0.029 \%$ & -0.008 & $100.000 \%$ \\
\hline 1270 & *33:03 & *39:01 & *12:01 & $0.006 \%$ & $-0.003 \%$ & -0.001 & $100.000 \%$ \\
\hline 1270 & *33:03 & *40:01 & ${ }^{*} 09: 01$ & $0.006 \%$ & $-0.048 \%$ & -0.009 & $100.000 \%$ \\
\hline 1270 & *33:03 & *40:02 & ${ }^{*} 04: 05$ & $0.006 \%$ & $-0.071 \%$ & -0.010 & $100.000 \%$ \\
\hline 1270 & $\star 33: 03$ & $* 40: 02$ & ${ }^{\star} 09 \cdot 01$ & $0.006 \%$ & $-0.075 \%$ & -0.011 & $100000 \%$ \\
\hline 1270 & *33:03 & *40:02 & *14:05 & $0.006 \%$ & $-0.005 \%$ & -0.003 & $100.000 \%$ \\
\hline 1270 & *33:03 & *40:03 & ${ }^{\star} 09: 01$ & $0.006 \%$ & $0.002 \%$ & 0.007 & $100.000 \%$ \\
\hline 1270 & *33:03 & *40:06 & ${ }^{\star} 04: 05$ & $0.006 \%$ & $-0.037 \%$ & -0.008 & $100.000 \%$ \\
\hline 1270 & *33:03 & *44:03 & ${ }^{\star} 04: 07$ & $0.006 \%$ & $0.004 \%$ & 0.007 & $100.000 \%$ \\
\hline 1270 & *33:03 & *44:03 & ${ }^{*} 04: 10$ & $0.006 \%$ & $-0.003 \%$ & -0.002 & $100.000 \%$ \\
\hline 1270 & *33:03 & *44:03 & ${ }^{*} 12: 02$ & $0.006 \%$ & $-0.002 \%$ & -0.001 & $100.000 \%$ \\
\hline 1270 & *33:03 & *44:03 & *14:06 & $0.006 \%$ & $0.000 \%$ & 0.000 & $100.000 \%$ \\
\hline 1270 & *33:03 & *46:01 & ${ }^{\star} 09: 01$ & $0.006 \%$ & $-0.043 \%$ & -0.009 & $100.000 \%$ \\
\hline 1270 & *33:03 & *48:01 & ${ }^{\star} 04: 05$ & $0.006 \%$ & $-0.021 \%$ & -0.008 & $100.000 \%$ \\
\hline 1270 & *33:03 & *48:01 & ${ }^{\star} 04: 10$ & $0.006 \%$ & $0.002 \%$ & 0.001 & $100.000 \%$ \\
\hline 1270 & *33:03 & *48:01 & ${ }^{\star} 09: 01$ & $0.006 \%$ & $-0.022 \%$ & -0.008 & $100.000 \%$ \\
\hline 1270 & *33:03 & *51:01 & ${ }^{*} 04: 10$ & $0.006 \%$ & $-0.008 \%$ & -0.004 & $100.000 \%$ \\
\hline 1270 & *33:03 & \begin{tabular}{|l|}
$* 51: 01$ \\
\end{tabular} & ${ }^{*} 08: 02$ & $0.006 \%$ & $-0.022 \%$ & -0.005 & $100.000 \%$ \\
\hline 1270 & *33:03 & \begin{tabular}{|l|}
$* 51: 01$ \\
\end{tabular} & ${ }^{*} 08: 03$ & $0.006 \%$ & $-0.050 \%$ & -0.007 & $100.000 \%$ \\
\hline 1270 & *33:03 & *52:01 & ${ }^{\star} 12: 01$ & $0.006 \%$ & $-0.023 \%$ & -0.006 & $100.000 \%$ \\
\hline 1270 & *33:03 & *54:01 & ${ }^{*} 01: 01$ & $0.006 \%$ & $-0.025 \%$ & -0.004 & $100.000 \%$ \\
\hline 1270 & *33:03 & *54:01 & *14:05 & $0.006 \%$ & $-0.005 \%$ & -0.002 & $100.000 \%$ \\
\hline 1270 & *33:03 & *55:02 & ${ }^{*} 04: 05$ & $0.006 \%$ & $-0.018 \%$ & -0.007 & $100.000 \%$ \\
\hline 1270 & *33:03 & *55:02 & ${ }^{*} 09: 01$ & $0.006 \%$ & $-0.019 \%$ & -0.008 & $100.000 \%$ \\
\hline 1270 & *33:03 & *55:02 & *12:01 & $0.006 \%$ & $-0.001 \%$ & 0.000 & $100.000 \%$ \\
\hline 1270 & *33:03 & *56:01 & *14:54 & $0.006 \%$ & $0.004 \%$ & 0.004 & $100.000 \%$ \\
\hline 1270 & *33:03 & *58:01 & ${ }^{*} 04: 03$ & $0.006 \%$ & $0.005 \%$ & 0.008 & $100.000 \%$ \\
\hline
\end{tabular}




\begin{tabular}{|c|c|c|c|c|c|c|c|}
\hline 1270 & $\star 33: 03$ & *58:01 & ${ }^{\star} 04: 10$ & $0.006 \%$ & $0.005 \%$ & 0.008 & $100.000 \%$ \\
\hline 1270 & *33:03 & $\star 58: 01$ & ${ }^{\star} 09: 01$ & $0.006 \%$ & $0.000 \%$ & -0.001 & $100.000 \%$ \\
\hline 1270 & *33:03 & *58:01 & *11:01 & $0.006 \%$ & $0.005 \%$ & 0.008 & $100.000 \%$ \\
\hline 1270 & $\star 33: 03$ & $\star 58: 01$ & $\star 12: 01$ & $0.006 \%$ & $0.004 \%$ & 0.007 & $100.000 \%$ \\
\hline 1270 & *33:03 & *58:01 & *12:02 & $0.006 \%$ & $0.005 \%$ & 0.008 & $100.000 \%$ \\
\hline 1270 & *33:03 & $\star 58: 01$ & *14:03 & $0.006 \%$ & $0.005 \%$ & 0.008 & $100.000 \%$ \\
\hline 1270 & *33:03 & ${ }^{*} 67: 01$ & *16:02 & $0.006 \%$ & $0.005 \%$ & 0.005 & $100.000 \%$ \\
\hline 1270 & *33:08 & *54:01 & *04:05 & $0.006 \%$ & $0.006 \%$ & 0.998 & $100.000 \%$ \\
\hline 1270 & *34:01 & *15:01 & *15:02 & $0.006 \%$ & $0.006 \%$ & 0.326 & $100.000 \%$ \\
\hline 1270 & *34:01 & *15:13 & $\star 12: 02$ & $0.006 \%$ & $0.006 \%$ & 1.005 & $100.000 \%$ \\
\hline 1270 & *34:01 & *40:02 & *15:02 & $0.006 \%$ & $0.006 \%$ & 0.326 & $100.000 \%$ \\
\hline 1270 & *66:01 & *41:02 & *07:01 & $0.006 \%$ & $0.006 \%$ & 1.005 & $100.000 \%$ \\
\hline 1270 & *68:01 & *39:05 & *04:07 & $0.006 \%$ & $0.006 \%$ & 1.005 & $100.000 \%$ \\
\hline 1270 & *68:02 & *14:02 & *15:01 & $0.006 \%$ & $0.006 \%$ & 1.005 & 100.00 \\
\hline
\end{tabular}

The data based on the pedigree study from 4,743 families (17,325 members).

aLinkage disequilibrium value

${ }^{\mathrm{b}}$ Relative linkage disequilibrium value

${ }^{\mathrm{C}}$ Cumulative coverage of the Japanese population by HLA homozygous donors 
Supplementary Table 9. Pla-iPSC clone list.

Source $\quad$ Gene expression

Differentiation

Copy

Clone Origin Age Sex Race Factors RT-PCR Microarray Bisulfite number Karyotyping HLA STR Teratoma Dopa RPE

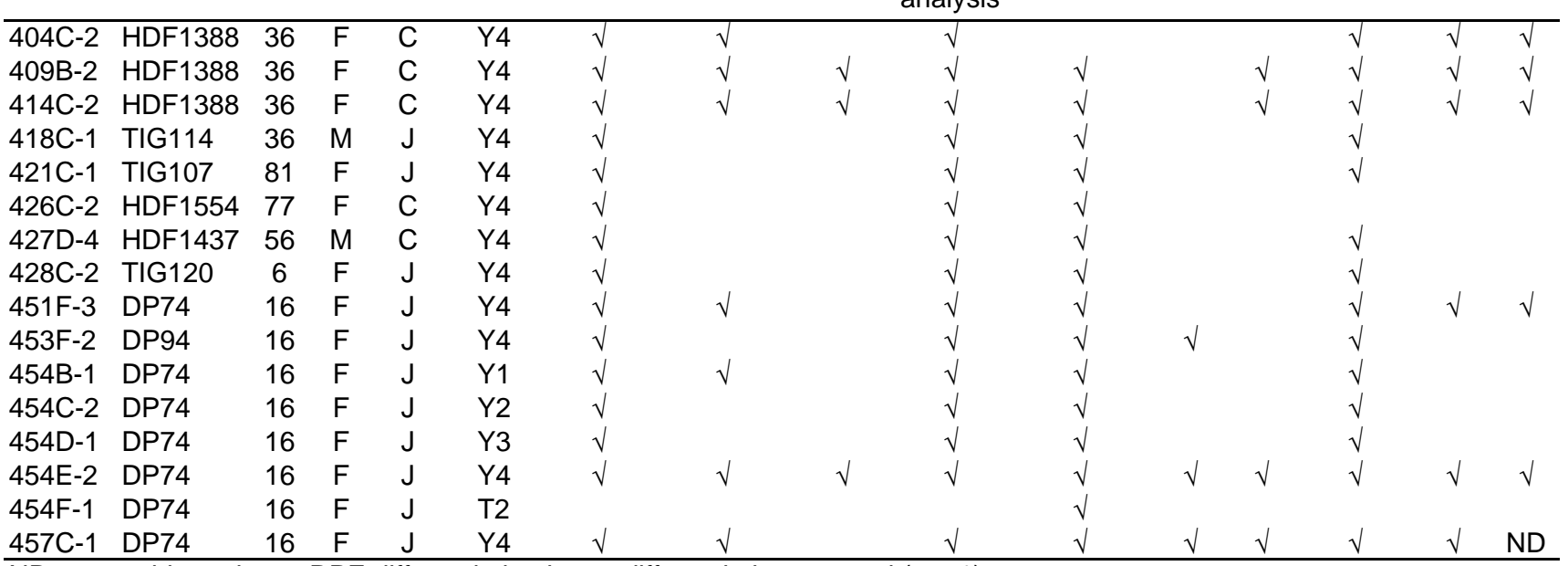

ND, we could not detect RPE differentiation in our differentiation protocol $(n=1)$. 
Supplementary Table 10. Primer list for quantitative PCR.

\begin{tabular}{|c|c|}
\hline OCT3/4 (CDS) & \begin{tabular}{|l|} 
CCC CAG GGC CCC ATT TTG GTA CC \\
ACC TCA GTT TGA ATG CAT GGG AGA GC
\end{tabular} \\
\hline OCT3/4 (pla) & CAT TCA AAC TGA GGT AAG GG \\
\hline & TAG CGT AAA AGG AGC AAC ATA G \\
\hline KLF4 (CDS) & ACC CAT CCT TCC TGC CCG ATC AGA \\
\hline & TTG GTA ATG GAG CGG CGG GAC TTG \\
\hline KLF4 (pla) & CCA CCT CGC CTT ACA CAT GAA GA \\
\hline & TAG CGT AAA AGG AGC AAC ATA G \\
\hline SOX2 (CDS) & TTC ACA TGT CCC AGC ACT ACC AGA \\
\hline & TCA CAT GTG TGA GAG GGG CAG TGT GC \\
\hline SOX2 (pla) & TTC ACA TGT CCC AGC ACT ACC AGA \\
\hline & TTT GTT TGA CAG GAG CGA CAA T \\
\hline$L-M Y C$ (CDS) & GCG AAC CCA AGA CCC AGG CCT GCT CC \\
\hline & CAG GGG GTC TGC TCG CAC CGT GAT G \\
\hline L-MYC (pla) & GGC TGA GAA GAG GAT GGC TAC \\
\hline & TTT GTT TGA CAG GAG CGA CAA T \\
\hline LIN28 (CDS) & AGC CAT ATG GTA GCC TCA TGT CCG C \\
\hline & TCA ATT CTG TGC CTC CGG GAG CAG GGT AGG \\
\hline LIN28 (pla) & AGC CAT ATG GTA GCC TCA TGT CCG C \\
\hline & TAG CGT AAA AGG AGC AAC ATA G \\
\hline$\overline{G A P D H}$ & ACC ACA GTC CAT GCC ATC AC \\
\hline & TCC ACC ACC CTG TTG CTG TA \\
\hline EBNA-1 & ATC AGG GCC AAG ACA TAG AGA TG \\
\hline & GCC AAT GCA ACT TGG ACG TT \\
\hline FBXO15 & $\begin{array}{l}\text { GCC AGG AGG TCT TCG CTG TA } \\
\text { AAT GCA CGG CTA GGG TCA AA }\end{array}$ \\
\hline
\end{tabular}

CDS; for detection of cording sequence.

pla; for detection of plasmid vector-derived expression. 